\title{
Residual platelet reactivity in atherosclerosis and its impact on patient outcomes
}

By

Lisa Renee Johnston

A thesis submitted to the Victoria University of Wellington in fulfilment of the requirements for the degree of Doctor of Philosophy

Victoria University of Wellington

2014 


\begin{abstract}
In this thesis, the role of platelets as potential drivers of both thrombotic and bleeding risk in patients with atherosclerosis and the influence of platelets on the immune system were investigated. The prevalence of high and low residual platelet reactivity phenotypes and the association of these phenotypes with thrombotic and bleeding risk in acute coronary syndrome (ACS) patients and patients with atherosclerotic vascular disease were examined. In ACS patients, the clinical factors that influence the residual platelet phenotype including the presence of genetic polymorphisms were tested. In addition to residual platelet reactivity, the utility of other cardiac biomarkers to predict perioperative and 1-year risk in vascular patients was also examined. To investigate the influence of platelets on the immune system, platelet-lymphocyte interactions in vascular patients and healthy volunteers were analysed.
\end{abstract}

In ACS patients treated with aspirin and clopidogrel, the prevalence of high on treatment residual platelet reactivity (as identified with the Multiplate assay), was common in a New Zealand population and high residual platelet reactivity levels could be significantly reduced with prasugrel treatment. This phenotype was predicted by the presence of diabetes, high platelet count and a low clopidogrel dose. It was also demonstrated that although the prevalence of loss of function and gain of function CYP2C19 alleles were high in our population, genotype had little influence on residual platelet reactivity levels in ACS patients. Furthermore, both phenotype and diabetes were significantly and independently associated with major adverse cardiovascular events (MACE) at 1 year. Genotype was not found to be a significant driver of risk. The incidence of major bleeding in this cohort was low and not predicted by platelet reactivity phenotype or genotype.

In stable vascular patients the study found the level of residual platelet reactivity on aspirin therapy was not significantly associated with MACE or bleeding following major elective surgery. However, baseline high sensitivity troponin T was the most predictive biomarker and an elevated preoperative level was significantly associated with an increased risk of MACE in both the short and long term. Bleeding was frequent 
and occurred early following surgery in the majority of patients and open abdominal aortic aneurysm repair surgery was the only predictor of bleeding.

Investigating the interactions between platelets and lymphocytes in atherosclerosis, the study found higher levels of platelet-T cell conjugates in patients with vascular disease compared to healthy controls. There was also interesting changes in the CD4 $\mathrm{T}$ cell activation phenotype with a switch from a predominant memory phenotype in healthy controls to an effector activation phenotype in vascular patients. A follow on study investigated the impact of platelets on CD4 T cells in more depth and found that platelets had a potent stimulatory effect on CD4 T cell differentiation and cytokine production ex vivo in healthy volunteers. Furthermore, the CD4 T cell stimulation provided by platelets was eliminated with in vivo prasugrel treatment. The influence of platelets on the immune system demonstrated in these two studies suggests that antiplatelet therapy may modulate not only thrombosis but also inflammation. 


\section{Acknowledgements}

No major achievement happens in isolation and clinical research in particular involves the work and support of many people. I owe a great deal of gratitude to these people, for the support, encouragement and sacrifices they have made to assist me over the past three years.

I would particularly like to thank all the staff in the Wellington Hospital Cardiology Department and Vascular Surgery Department. As a Biomedical science student with little clinical experience, the expertise, patience and help I have received from the clinicians and nurses has made the introduction to translational research all the more enjoyable. I would also like to thank all of the patients involved in the studies I have conducted. Their co-operation and patience during recruitment and sample collection whilst dealing with an often-stressful health scare has been greatly appreciated.

I would like to thank my supervisors Dr Scott Harding, A/Prof Peter Larsen and A/Prof Anne La Flamme. They have all provided invaluable support and guidance throughout my thesis. Their individual skills and expertise are vast and together they have provided helpful troubleshooting and encouragement.

Thank you to all the people in the clinical research lab: Ana, Melanie, Charlotte, Aimee, Bijia, Kathryn, Richard, and Bev; and also thanks to the LAF lab group. Collectively these people have provided a rich and enjoyable work environment. I would particularly like to thank Ana and Melanie for the important early lunch breaks and support through tough times. Thank you to Ana, Richard and Michael for help with patient recruitment and sample processing. Thank you to cardiology registrars lan Webb and Nadim Shah for their help with procedural details for patients undergoing angiography and the research nurses Bev and Di for their hard work to complete all patient follow up.

Thanks to my wonderful family for their support and encouragement throughout. There have been some tough times in the last few years, which would have been 
overwhelming without the comfort of family. In particular thank you mum, for the hours of proofreading and numerous phone calls.

Finally I would like to thank my partner. Jason has been incredibly tolerant and supportive, and has made many sacrifices to help me achieve my goals. 


\section{Table of Contents}

Abstract iii

Acknowledgements v v

Table of Contents vii

List of Figures xi

List of Tables xiii

Publications arising from thesis xiv

Abbreviations $\quad$ xv

1 Chapter 1 - Introduction 1

1.1 Introduction Overview 2

1.2 Atherosclerosis 3

1.2.1 Pathophysiology 3

$\begin{array}{ll}1.2 .2 & \text { Immune system } \\ 1.2 .3 & 5\end{array}$

$\begin{array}{ll}1.2 .3 \text { Acute Coronary Syndromes } & 7\end{array}$

$\begin{array}{ll}1.2 .4 \text { Treatment } & 10\end{array}$

$\begin{array}{lll}1.2 .5 & \text { Peripheral Vascular Disease } & 11\end{array}$

1.2.6 Carotid Artery Disease 11

1.2.7 Lower Extremity Peripheral Vascular Disease 12

$\begin{array}{ll}\text { 1.2.8 Abdominal Aortic Aneurysm } & 13\end{array}$

$\begin{array}{ll}1.3 \text { Platelets } & 14\end{array}$

$\begin{array}{ll}\text { 1.3.1 Function in Hemostasis } & 14\end{array}$

$\begin{array}{ll}\text { 1.3.2 Platelets in Atherosclerosis } & 15\end{array}$

$\begin{array}{ll}\text { 1.3.3 Plaque initiation and development } & 15\end{array}$

$\begin{array}{ll}\text { 1.3.4 Platelets in thrombosis } & 19\end{array}$

1.4 Antiplatelet agents and Non-Response 20

$\begin{array}{ll}\text { 1.4.1 Aspirin therapy } & 24\end{array}$

$\begin{array}{lll}1.4 .2 & \text { Aspirin HOTPR } & 26\end{array}$

$\begin{array}{lll}\text { 1.4.3 P2Y12 Inhibitors } & 27\end{array}$

$\begin{array}{ll}1.4 .4 \text { Clopidogrel } & 28\end{array}$

1.4.5 Clopidogrel HOTPR 32

1.4.6 Clopidogrel LOWPR $\quad 35$

$\begin{array}{lll}1.4 .7 & \text { Prasugrel } & 35\end{array}$

$\begin{array}{lll}1.4 .8 & \text { Ticagrelor } & 37\end{array}$

1.4.9 Novel P2Y12 inhibitors 38

1.4.10 Other platelet inhibitors under clinical trial $\quad 39$

1.4.11 HOTPR and LOWPR risk factors 41

$\begin{array}{ll}1.4 .12 & \text { Genetic Factors }\end{array}$

$\begin{array}{lll}1.4 .13 & \text { Drug-Drug Interactions } & 43\end{array}$

$\begin{array}{lll}1.4 .14 & \text { Diabetes } & 44\end{array}$

$\begin{array}{lll}1.4 .15 & \text { Obesity } & 46\end{array}$

1.4.16 Renal Insufficiency 46

1.5 Platelet Function Testing 48

1.5.1 Platelet function assays $\quad 50$

1.5.2 Personalised antiplatelet therapy 52

1.6 Overall aims and objectives 54

2 Chapter 2 - Measurement of platelet reactivity 57

2.1 Introduction 58

2.2 Methods 59

$\begin{array}{ll}2.2 .1 & \text { Study Population }\end{array}$ 
2.2.2 Blood sampling and platelet function testing 59

2.2.3 Experimental Design $\quad 62$

2.2.4 Statistics 63

2.3 Results 64

2.3.1 Analytical Precision $\quad 64$

2.3.2 Choice of anticoagulant 66

2.3.3 Effect of time delay between sampling and testing 67

$\begin{array}{lll}\text { 2.3.4 Manual Pipetting } & 68\end{array}$

2.4 Discussion $\quad 69$

2.5 Limitations 71

2.6 Conclusion 72

3 Chapter 3 - Platelet reactivity in Acute Coronary Syndromes 73

3.1 Introduction 74

3.2 Methods $\quad 75$

3.2.1 Study Population: $\quad 75$

3.2.2 Data Collection $\quad 75$

3.2.3 Blood Collection and Platelet Function testing 75

$\begin{array}{ll}3.2 .4 & \text { Definitions } \\ 3 & 76\end{array}$

$\begin{array}{ll}3.2 .5 & \text { Statistical Analysis } \\ \end{array}$

$\begin{array}{lll}3.3 & \text { Results } & 77\end{array}$

3.3.1 Baseline demographics and prevalence of HOTPR 77

$\begin{array}{ll}\text { 3.3.2 Predictors of HOTPR } & 79\end{array}$

3.3.3 Effectiveness of prasugrel on HOTPR 83

3.4 Discussion 84

3.5 Limitations 86

3.6 Conclusion $\quad 87$

4 Chapter 4: Platelet reactivity genotype and phenotype 89

$\begin{array}{ll}4.1 & \text { Introduction } \\ 4.2 & 90\end{array}$

$\begin{array}{ll}4.2 \text { Methods } & 91\end{array}$

$\begin{array}{ll}\text { 4.2.1 Study Population } & 91\end{array}$

$\begin{array}{ll}\text { 4.2.2 Data Collection } & 91\end{array}$

4.2.3 Blood Collection and Platelet function testing 92

4.2.4 Isolation of Genomic DNA 92

4.2.5 Validation of CYP2C19*2 and CYP2C19*17 HRM Assays 93

4.2.6 Genotyping of CYP2C19*2 and CYP2C19*17 93

4.2.7 Restriction Fragment Length Polymorphism (RFLP) and Sanger
Sequencing

$\begin{array}{lll}\text { 4.2.8 Definitions } & 95\end{array}$

4.2.9 Statistical analysis $\quad 95$

$\begin{array}{lll}4.3 & \text { Results } & 97\end{array}$

$\begin{array}{ll}\text { 4.3.1 Demographics } & 97\end{array}$

4.3.2 Predictors of platelet reactivity 98

4.3.3 Multivariate analysis of platelet reactivity predictors 103

$\begin{array}{lll}4.4 & \text { Discussion } & 104\end{array}$

4.5 Limitations 109

$\begin{array}{lr}4.6 & \text { Conclusion } \\ \end{array}$

5 Chapter 5: Platelet reactivity and outcomes in Acute Coronary Syndromes 111

$\begin{array}{lll}5.1 & \text { Introduction } & 112\end{array}$

5.2 Methods 112

5.2.1 Study population 112

5.2.2 Data collection 113

$\begin{array}{ll}\text { 5.2.3 Platelet function testing and Genotyping } & 113\end{array}$ 
5.2.4 End points and definitions 113

5.2.5 Statistics 114

$\begin{array}{lll}5.3 \text { Results } & 115\end{array}$

5.3.1 Patient outcome 115

5.3.2 ROC curve analysis of residual platelet reactivity and outcome 116

$\begin{array}{ll}\text { 5.3.3 Predictors of MACE } & 119\end{array}$

$\begin{array}{ll}\text { 5.3.4 Predictors of bleeding } & 121\end{array}$

5.3.5 Subgroup analysis of $\mathrm{PCl}$ cohort 122

$\begin{array}{lll}5.4 \text { Discussion } & 125\end{array}$

$\begin{array}{ll}5.4 .1 & \text { Limitations } \\ 5.4 .2 & 131\end{array}$

$\begin{array}{ll}5.4 .2 \text { Conclusion } & 131\end{array}$

6 Chapter 6: Platelet reactivity, biomarkers and outcome following vascular

$\begin{array}{ll}\text { surgery } & 133\end{array}$

$\begin{array}{lll}6.1 & 134\end{array}$

6.2 Methods 135

6.2.1 Study Population 135

$\begin{array}{ll}\text { 6.2.2 Data Collection } & 135\end{array}$

6.2.3 Study design, ECG and blood collection 135

6.2.4 Platelet function testing and biomarker analysis 136

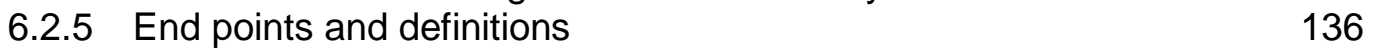

$\begin{array}{ll}\text { 6.2.6 Statistical analysis } & 137\end{array}$

$\begin{array}{lll}6.3 & \text { Results } & 139\end{array}$

6.3.1 Demographics 139

6.3.2 Perioperative measurement of hs-TnT 142

6.3.3 Patient outcomes following vascular surgery 143

$\begin{array}{ll}\text { 6.3.4 Short term outcomes } & 143\end{array}$

6.3.5 Long term outcomes 144

6.3.6 Predictors of adverse outcomes in vascular patients 145

$\begin{array}{lll}6.4 & \text { Discussion } & 157\end{array}$

6.5 Limitations 162

6.6 Conclusion 163

7 Chapter 7: Platelet-T cell interactions $\quad 165$

$\begin{array}{lll}7.1 & 166\end{array}$

$\begin{array}{llr}7.2 & \text { Methods } & 167\end{array}$

$\begin{array}{ll}\text { 7.2.1 Study population } & 167\end{array}$

7.2.2 Blood collection and antibody staining 168

$\begin{array}{lll}7.2 .3 & \text { Flow cytometry } & 168\end{array}$

$\begin{array}{lll}7.2 .4 & \text { Statistics } & 172\end{array}$

$\begin{array}{llr}7.3 & \text { Results } & 173\end{array}$

$\begin{array}{lll}\text { 7.3.1 Demographics } & 173\end{array}$

$\begin{array}{ll}\text { 7.3.2 Platelet-T cell conjugates } & 173\end{array}$

$\begin{array}{lll}\text { 7.3.3 CD4 T cell interactions } & 174\end{array}$

$\begin{array}{lll}\text { 7.3.4 CD8 T cell interactions } & 176\end{array}$

$\begin{array}{ll}\text { 7.3.5 Natural Killer T cells } & 177\end{array}$

$\begin{array}{lll}7.4 & \text { Discussion } & 179\end{array}$

$\begin{array}{lll}7.5 & \text { Limitations } & 184\end{array}$

$\begin{array}{lll}7.6 & \text { Conclusion } & 185\end{array}$

8 Chapter 8 - The effect of platelets on CD4 T cell differentiation 187

8.1 Introduction 188

8.2 Methods 189

8.2.1 Study population 189

8.2.2 Study design and drug treatment 189

$\begin{array}{ll}\text { 8.2.3 Blood sampling and Multiplate testing } & 190\end{array}$ 
8.2.4 Peripheral blood mononuclear cell (PBMC) isolation 190

8.2.5 CD4 T cell isolation 191

8.2.6 Isolation of autologous platelets 191

8.2.7 Cell culture 192

8.2.8 CD4 T cell phenotyping 192

8.2.9 Flow cytometry 193

8.2.10 Quantification of IFN-ץ production by ELISA 198

$\begin{array}{lll}\text { 8.2.11 Statistics } & 198\end{array}$

$\begin{array}{lll}8.3 \text { Results } & 199\end{array}$

8.3.1 Period effect and treatment-period interaction analysis 199

8.3.2 Platelet function following placebo and prasugrel therapy 201

8.3.3 IFN- $\gamma$ production and CD4 T cell differentiation at baseline 201

8.3.4 The effect of prasugrel on IFN- $\gamma$ production and CD4 T cell differentiation 204

$\begin{array}{lll}8.4 & \text { Discussion } & 207\end{array}$

8.5 Limitations 211

8.6 Conclusion 212

9 Chapter 9 - Summary and future directions 213

$\begin{array}{lll}9.1 & \text { Summary } & 214\end{array}$

$\begin{array}{lll}9.2 & \text { Limitations } & 215\end{array}$

9.3 Clinical implications and future directions 216

10 References $\quad 221$

11 Appendices $\quad 247$

11.1 Appendix 1-Buffers and Solutions 248

11.2 Appendix 2-Antibody information 249

11.3 Appendix 3-Supplementary graphs for chapter 8 


\section{List of Figures}

Figure 1-1 Progression of atherosclerotic plaques

Figure 1-2 Spectrum of Acute Coronary Syndromes (ACS)

Figure 1-3 Role of platelets in the vascular inflammatory response associated with atherosclerosis

Figure 1-4 Antiplatelet agent targets to prevent ischaemic events

Figure 1-5 Aspirin effects via the inhibition of COX activity.

Figure 1-6 Metabolism of clopidogrel

Figure 1-7 Metabolism of Prasugrel

Figure 1-9 Laboratory measurement of on treatment platelet reactivity. 48

Figure 1-10 Therapeutic window of P2Y12 blockade 51

Figure 2-1 Multiplate Analyser System 60

Figure 2-2 Multiplate electrode impedance aggregometry 61

Figure 2-3 Aggregation over time in Multiplate test 62

Figure 2-4 Coefficient of variance (CV) in platelet aggregation using standard Multiplate protocol with ADP stimulation.

Figure 2-5 Coefficient of variance (CV) in platelet aggregation using standard Multiplate protocol with Arachadonic Acid (AA) stimulation.

Figure 2-6 Effect of anticoagulant on variance in platelet aggregation.

Figure 2-7 Effect of time delay from collection to assay performance on platelet aggregation.

Figure 2-8 Coefficient of variance $(\mathrm{CV})$ in platelet aggregation using calibrated manual pipette and ADP stimulation

Figure 3-1 Patient ethnicity and platelet reactivity. $\quad 79$

Figure 3-2 Diabetes and platelet reactivity $\quad 80$

Figure 3-3 Diabetes, ethnicity and platelet reactivity 81

Figure 3-4 Clopidogrel dose and platelet reactivity. 82

Figure 3-5 Platelet reactivity pre and post prasugrel administration 83

Figure 4-1 High resolution melting curve assay 93

Figure 4-2 Clinical predictors of platelet reactivity $\quad 99$

Figure 4-3 CYP2C19*2 loss of function allele (LOF) and platelet reactivity 100

Figure 4-4 CYP2C19*17 Gain of Function (GOF) allele and platelet reactivity 101

Figure 4-5 Ethnicity and platelet reactivity 102

$\begin{array}{ll}\text { Figure 5-1 ROC curve analysis of residual platelet reactivity and MACE } & 116\end{array}$

$\begin{array}{ll}\text { Figure 5-2 Residual platelet reactivity and HOTPR cut-points } & 118\end{array}$

Figure 5-3 ROC curve analysis of residual platelet reactivity and major bleeding 118

Figure 5-4 ROC curve analysis of residual platelet reactivity and all bleeding 119

$\begin{array}{lr}\text { Figure 5-5 Univariate predictors of MACE } & 120\end{array}$

Figure 5-6 Multivariate predictors of MACE 120

Figure 5-7 Univariate predictors of bleeding 121

Figure 5-8 ROC curve analysis of residual platelet reactivity and MACE in PCI subgroup 123

Figure 5-9 Univariate predictors of MACE in the PCl subgroup 124

Figure 5-10 Multivariate analysis of univariate MACE predictors in the PCl subgroup 124

Figure 6-1 Variability of baseline biomarkers 141

$\begin{array}{lr}\text { Figure 6-2 Hs-TnT measurement perioperatively } & 143\end{array}$

Figure 6-3 Short term timing of events post surgery 144

$\begin{array}{ll}\text { Figure 6-4 Timing of events from } 30 \text { days to } 1 \text { year post discharge } & 145\end{array}$

$\begin{array}{ll}\text { Figure 6-5 Predictors of MACE at } 1 \text { year } & 146\end{array}$

$\begin{array}{ll}\text { Figure 6-6 Multivariate analysis of predictors of all MACE } & 146\end{array}$

Figure 6-7 ROC curve analysis of residual platelet reactivity and MACE $\quad 147$

$\begin{array}{ll}\text { Figure 6-8 Predictors of bleeding at } 1 \text { year } & 148\end{array}$

$\begin{array}{ll}\text { Figure 6-9 ROC curve analysis of residual platelet reactivity and bleeding } & 149\end{array}$

Figure 6-10 Predictors of short term MACE 150

Figure 6-11 Multivariate analysis of short term MACE predictors 150

$\begin{array}{ll}\text { Figure 6-12 Predictors of short term bleeding } & 151\end{array}$

Figure 6-13 Platelet reactivity and short term events 152

Figure 6-14 Predictors of patients at risk of both short term MACE and bleeding 153 
Figure 6-15 Multivariate analysis of predictors of patients at risk of short term MACE and bleeding

Figure 6-16 Predictors of long term MACE

Figure 6-17 Multivariate analysis of predictors of long term MACE 155

$\begin{array}{lr}\text { Figure 6-18 Predictors of long term bleeding } & 155\end{array}$

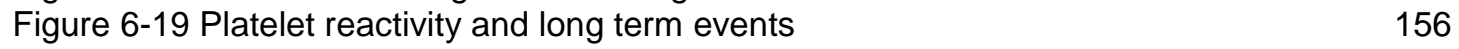

$\begin{array}{ll}\text { Figure 7-1 Gating strategies to identify CD8 and CD4 platelet-T cell conjugates. } & 170\end{array}$

Figure 7-2 Gating strategy to identify activation phenotype of CD4 and CD8 T cells 171

Figure 7-3 Gating strategies to identify T regulatory (Treg) cells and Natural Killer T cells (NKT).

Figure 7-4 Platelet-T cell conjugates 174

$\begin{array}{ll}\text { Figure 7-5 CD4 T cell interactions with platelets } & 175\end{array}$

$\begin{array}{ll}\text { Figure 7-6 T regulatory (Treg) cell interactions with platelets } & 176\end{array}$

$\begin{array}{ll}\text { Figure 7-7 CD8 T cell interactions with platelets. } & 177\end{array}$

$\begin{array}{ll}\text { Figure 7-8 Natural Killer T (NKT) cell interactions with platelets } & 178\end{array}$

$\begin{array}{lr}\text { Figure 8-1 Study cross over protocol } & 190\end{array}$

$\begin{array}{ll}\text { Figure 8-2 Identification of CD4 T cells } & 194\end{array}$

$\begin{array}{ll}\text { Figure 8-3 Identification of T helper (Th)1 cells } & 195\end{array}$

Figure 8-4 Identification of Thelper (Th)17 cells 196

Figure 8-5 Identification of T regulatory (Treg) cells 197

Figure 8-7 Marked reduction in platelet function following prasugrel therapy 201

Figure 8-8 The addition of platelets to anti-CD3/CD28 stimulated CD4 T cells enhances the production of IFN- and Th1 and Th17 differentiation. 203

Figure 8-9 No difference in isolated CD4 T cell IFN-y production following placebo therapy compared to baseline in all culture conditions

Figure 8-10 Prasugrel therapy abolishes the enhancement of isolated CD4 T cell IFN- $\gamma$ production and Th1 and Th17 differentiation by platelets

Figure 11-1 No difference in isolated CD4 T cell differentiation following placebo therapy compared to baseline in all culture conditions 


\section{List of Tables}

Table 1-1 Factors and conditions associated with increased risk for an Acute Coronary Syndrome

Table 1-2 Guidelines for Antiplatelet therapy in patients with CAD

Table 1-4 Major novel antiplatelet agents in development 40

Table 1-5 Mechanisms contributing to platelet dysfunction in diabetes 46

Table 1-6 Platelet Function Assays $\quad 49$

Table 3-1 Patient baseline demographics, clinical characteristics and laboratory data $\quad 78$

$\begin{array}{ll}\text { Table 3-2 Patient Ethnicity and platelet reactivity } & 79\end{array}$

Table 3-3 Diabetes and platelet reactivity $\quad 80$

Table 3-4 Diabetes, ethnicity and platelet reactivity 81

Table 3-5 Clopidogrel dose and platelet reactivity 82

Table 4-1 Primer Sequences $\quad 94$

Table 4-2 Patient baseline demographics $\quad 97$

Table 4-3 Multivariate Linear Regression Coefficients 103

$\begin{array}{lr}\text { Table 5-1 Coronary angiogram procedure variables } & 115\end{array}$

$\begin{array}{ll}\text { Table 5-2 ROC curve coordinates } & 117\end{array}$

Table 5-3 PCl procedure variables 122

Table 5-4 PCI ROC curve coordinates 123

Table 6-1 Patient baseline demographics 139

$\begin{array}{ll}\text { Table 6-2 Admission medications } & 140\end{array}$

Table 6-3 Baseline measurement of biomarkers 141

$\begin{array}{ll}\text { Table 6-4 Surgery details } & 142\end{array}$

$\begin{array}{lr}\text { Table 7-1 Platelet-T cell conjugates antibody panels } & 169\end{array}$

Table 8-1 Group A: Drug (prasugrel) followed by placebo $n=6 \quad 200$

Table 8-2 Group B: Placebo followed by drug (prasugrel) $n=6 \quad 200$ 


\section{Publications arising from thesis}

The following papers were published before completion of the thesis:

Johnston LR, Larsen PD, La Flamme AC, Harding SA. Methodological considerations for the assessment of ADP induced platelet aggregation using the Multiplate ${ }^{\circledR}$ analyser. Platelets. 2013;24(4):303-ㅇ- 7 . doi:10.3109/09537104.2012.694086. Epub 2012 Jun 11. PMID:22686487

Johnston LR, Larsen PD, La Flamme AC, Michel JM, Simmonds MB, Harding SA. Suboptimal response to clopidogrel and the effect of prasugrel in acute coronary syndromes. Int J Cardiol. 2013 Aug 10;167(3):995- ${ }^{\circ}$-9. doi: 10.1016/j.ijcard.2012.03.080. Epub 2012 Apr 1. PMID: 22465351 


\section{Abbreviations}

AA - arachadonic acid

$A A A$ - abdominal aortic aneurysm

ABCB1 - ATP binding cassette sub family $B$ member 1

ACS - acute coronary syndrome

ADP - adenosine diphosphate

APC - antigen presenting cell

$A U$ - aggregation units

$A U$ - aggregation units

AUC - area under the curve

BARC - bleeding academic research consortium

$\mathrm{BMI}$ - body mass index

$\mathrm{CABG}$ - coronary artery bypass graft

$\mathrm{CaCl} 2$ - Calcium chloride

CAD - coronary artery disease

CLI - critical limb ischaemia

COPD - chronic obstructive pulmonary disease

COX - cyclooxygenase

CRP - c-reactive protein

CT - computed tomography

CTCM - complete T cell media

$\mathrm{CV}$ - coefficient of variation

CVA - cerebrovascular attack

CYP - cytochrome

DAPT - dual antiplatelet therapy

DM - diabetes mellitus

DNA - deoxyribonucleic acid

EDTA - ethylenediaminetetraacetic acid

eGFR - estimated glomerular filtration rate

ELISA - enzyme-linked immunosorbent assay

FSC - forward scatter

GOF - gain of function

GP - glycoprotein

HOTPR - high on treatment platelet reactivity

$\mathrm{HRM}$ - high-resolution melting assay

Hs-TnT - high sensitivity troponin T

IFN- $\gamma$ - interferon- $\gamma$

IL - interleukin

IONO - ionomycin

LBBB - left bundle branch block

LDL - low-density lipids

LOF - loss of function

LOWPR - low on treatment platelet reactivity

LTA - light transmission aggregometry

MACE - major adverse cardiovascular event

MCP-1 - monocyte chemoattractant protein-1

$\mathrm{MI}$ - myocardial Infarction 
MMP - matrix-metalloproteinase

NK T cells - natural killer T cells

NO - nitric oxide

NSAID - nonsteroidal anti-inflammatory drugs

NSTEMI - non ST-segment elevation myocardial infarction

NT-proBNP - N terminal propeptide brain natriuretic peptide

OR - odds ratio

$\mathrm{PAF}$ - platelet activating factor

PAR-1 - pro-tease activated receptor 1

PBMC - peripheral blood mononuclear cell

PBS - phosphate buffered saline

$\mathrm{PCl}$ - percutaneous coronary intervention

$\mathrm{PCR}$ - polymerase chain reaction

PDGF - platelet-derived growth factor

PF4 - platelet factor 4

PFA - paraformaldehyde

PMA - phorbol myristate acetate

PON-1 - paraoxonase-1

$\mathrm{PPI}$ - proton pump inhibitor

PRU - platelet reactivity units

PSGL-1 - P-selectin glycoprotein ligand 1

PVD - peripheral vascular disease

RBC - red blood cells

RFLP - restriction fragment length polymorphism

ROC - receiver operator characteristic curve analysis

$\mathrm{RT}$ - room temperature

SD - standard deviation

SEM - standard error of the mean

SNP - single nucleotide polymorphism

SSC - side scatter

STEMI - ST-segment elevation myocardial infarction

TCR $-\mathrm{T}$ cell receptor

TF - tissue factor

TGF $\beta$ - transforming growth factor $\beta$

Th1 - type $1 \mathrm{~T}$ helper cell

Th17 - type $17 \mathrm{~T}$ helper cell

Th2 - type $2 \mathrm{~T}$ helper cell

TIA - transient ischemic attacks

TNF - tumor necrosis factor

Treg - $T$ regulatory cells

TXA2 - thromboxane A2

UA - unstable angina

VASP - vasodilator-stimulated phosphoprotein

VWF - von Willebrand factor

$\mathrm{w} / \mathrm{t}$ - wild type 
1 Chapter 1 - Introduction 


\subsection{Introduction Overview}

Cardiovascular disease is both the current and projected number one cause of mortality and morbidity in the developed world. The impact of this disease is even greater in New Zealand (NZ) with 6000 patients dying from an Acute Coronary Syndrome (ACS) or "heart attack' every year and a disproportionate number of patients represented by Maori or Pacific Island ethnicities. Atherosclerosis is the principle underlying pathophysiology for the majority of cardiovascular disease and the platelet plays a central role in the progression and destabilisation of atherosclerosis. This is highlighted by the frontline treatment of ACS with antiplatelet agents such as aspirin and clopidogrel. The use of these treatments has been shown to be highly effective in reducing rates of death, recurrent myocardial infarction (MI), ischaemic stroke and stent thrombosis following an ACS. However, it is now recognised that the response to aspirin and clopidogrel is highly variable with patients lying somewhere on a spectrum of on treatment platelet reactivity. High on treatment platelet reactivity (HOTPR) results in an inadequate inhibition of platelets, which has been associated with an increased risk of ischaemic events. Low on treatment platelet reactivity (LOWPR) at the other end of the spectrum, leads to a significant decrease in clotting ability which has been linked to an increased risk of major bleeding. This variability suggests the presence of a therapeutic window when treating patients with aspirin and clopidogrel where both ischaemic and bleeding risk is reduced. Identifying where a patient sits on this platelet reactivity spectrum has been made possible by platelet function assays that test a patients' response to agonist stimulation. More potent new antiplatelet agents have the potential to decrease on treatment platelet reactivity and in the right patient population group guided by platelet function testing, reduce ischemic events. 


\subsection{Atherosclerosis}

\subsubsection{Pathophysiology}

Atherosclerosis is an inflammatory disease and the main cause of coronary artery and vascular disease. Atherosclerotic lesions develop in the intima of arteries with an asymmetrical pattern of thickening and are characterised by the presence of lipids, immune cells, connective tissue elements, endothelial and smooth muscle cells (1). The progression of simple lesions or fatty streaks to complex vulnerable plaques is shown in Figure 1-1.

Fatty streaks are comprised largely of lipid-laden macrophages and $\mathrm{T}$ cells accumulating beneath the endothelium (2) and preferentially distribute at areas in the vasculature where blood flow is low and oscillatory or "turbulent"(3). This turbulent flow limits the exposure of endothelial cells to nitric oxide in the blood, which leads to an increase in endothelial permeability, leukocyte adhesion and endothelial cell death supporting initiation and progression of atherosclerotic lesions (4). Oxidization of lowdensity lipids (LDL) in the intima leads to the release of phospholipids that can also activate endothelial cells and cause dysfunction (5). Therefore both hemodynamic strain and lipid oxidisation contribute to initiating inflammation in the artery. Platelets are one of the first cells to arrive at the site of endothelial activation and have been shown to participate in recruitment and aiding infiltration of leukocytes at the site of injury (6). The prevalence of these fatty streaks is high in the general population and can be found in young patients. They are subclinical and never cause symptoms but can progress on to complex lesions or eventually disappear (7). 


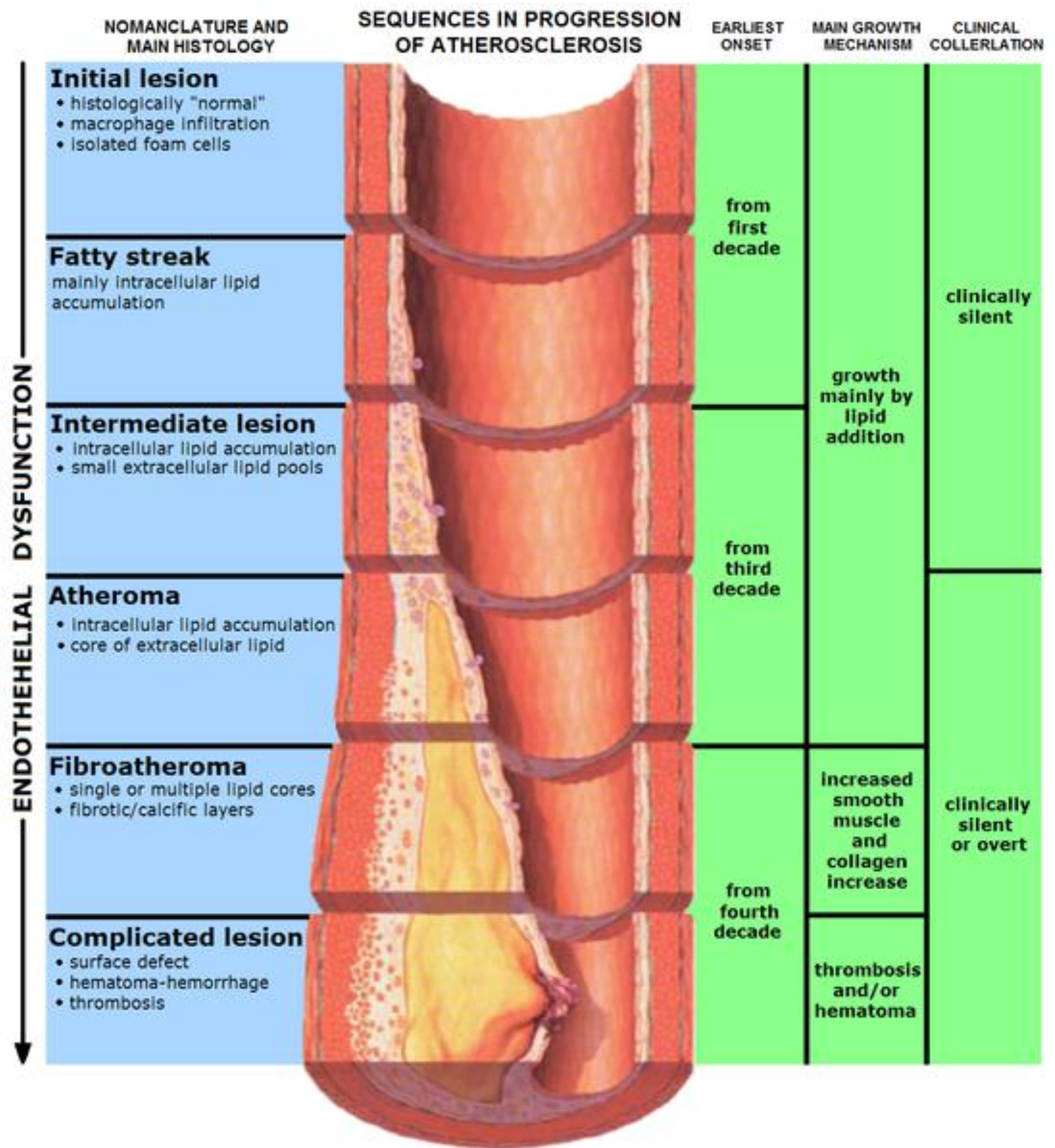

Figure 1-1 Progression of atherosclerotic plaques

The schematic depicts the sequences involved in the progression of atherosclerosis. These include the main histology findings associated with progression of lesions, the onset of each stage according to a patients age, the main growth mechanism involved at each stage and the clinical manifestation of each stage. With progression of atherosclersosis there is also an increase in endothelial dysfunction and complexity of the lesion. Image reproduced with permission from Creative Commons Attribution-Share Alike 3.0 Unported. 
As lesions develop, a core of foam cells and extracellular lipid droplets expands with a smooth muscle cell and collagen-rich matrix forming the surrounding cap. This process involves a mass infiltration of immune cells into the plaque including monocytes, $T$ cells and dendritic cells creating a rich pro-inflammatory milieu of cytokines and growth factors $(8,9)$. These cells preferentially deposit at the shoulder of plaques with a necrotic core of debris, lipids and dead cells (8). As these lesions develop and expand in size, the artery enlarges to compensate for the extra thickness of the atheroma and maintain flow in the lumen of the artery. However, if this enlargement of the artery progresses beyond the thickness of the atheroma, then an aneurysm is created (10). Stenosis is a relatively late event in the atherosclerotic process and is often a result of repeated plaque rupture and fibrous remodelling.

\subsubsection{Immune system}

The immune system is involved in both the initiation and progression of atherosclerotic plaques. Not only are immune cells present in large numbers at the early stages, their effector cells can accelerate or modulate progression and exacerbation of the local inflammatory environment can elicit an ACS or stroke. One of the critical elements for the development of atherosclerosis is the production of macrophage colony stimulating factor (M-CSF) in the inflamed intima that induces the differentiation of monocytes to macrophages as they enter the plaque (11). Differentiation induces the up-regulation of scavenger receptors and toll-like receptors on macrophages, which allows recognition and internalisation of oxidized $\operatorname{LDL}(12,13)$. The scavenger receptors function to destroy internalised LDL but if accumulation is too great, macrophages can be transformed into the prototypical foam cell. In contrast, toll-like receptor internalisation of oxidised LDL can initiate signal cascades leading to cell activation (13). This activation of macrophages induces the release of pro-inflammatory cytokines, proteases and reactive oxygen species (7). These two processes promote cell death, further infiltration of immune cells and weakening of the surrounding collagenous structure.

Large populations of $\mathrm{CD} 4+\mathrm{T}$ cells take residence in atherosclerotic plaques. Their infiltration is in response to the presentation of oxidised LDL and heat shock protein 


\section{INTRODUCTION}

60 antigens by trafficking macrophages and dendritic cells in the blood (14-16). The interaction between $\mathrm{T}$ cell and antigen causes an activation cascade in the $\mathrm{T}$ cell resulting in the expression of a set of cytokines, cell-surface molecules and enzymes. The type 1 helper (Th1) response is the predominant phenotype of CD4 T cells in the plaque resulting from the Th1 promoting cytokines (rather than a Th2) present during activation in plaques $(17,18)$. Th1 effector cells produce the macrophage activating cytokine interferon- $\gamma$ (IFN- $\gamma$ ) that in turn improves the macrophages' antigen presenting ability and augments synthesis of pro-inflammatory cytokines interleukin1 (IL-1) and tumour necrosis factor (TNF) (19). The synergetic action of these cytokines instigates the production of many inflammatory and cytotoxic molecules in macrophages and vascular cells promoting atherosclerosis (16).

The Th2 phenotype, classically an allergy response cell, has shown to be largely antiinflammatory but may also be pro-inflammatory in atherosclerosis. The Th17 phenotype, characterised by the secretion of IL-17, has more recently been recognized to be present and play a role in atherosclerotic plaques. Similarly to Th2 CD4 T cells, the impact of Th17 cell response in atherogensis is controversial (20-22). T regulatory (Treg) cells produce the anti-inflammatory IL-10 and TGF $\beta$ and are found in low numbers in atherosclerotic plaques (23). Treg cell numbers and IL-10 levels are severely decreased in patients experiencing an ACS (24) and in mouse models, the deficiency of these cells is linked to increased atherogenesis and lesion inflammation (25). Furthermore, increased Treg presence and stimulation of this phenotype has shown to reduce lesion formation and attenuate atherosclerosis (26-28). Natural Killer T cells and CD8+ T cells are also present in atherosclerotic plaques but tend to be found in early plaque development and in much smaller numbers $(16,29)$. Both cell types are pro-atherogenic in their activity (30). All major T cell lineages are present during atherogenesis and destabilisation of the balance between pro and antiinflammatory phenotypes drive the development of vulnerable plaques. 


\subsubsection{Acute Coronary Syndromes}

Despite the chronic nature of atherosclerosis, thrombotic complications or Acute Coronary Syndromes (ACS) occur suddenly and often without warning. The traditional view of infarction is the progressive luminal narrowing of a coronary artery from continued growth of smooth muscle cells in the plaque which a small platelet thrombus could occlude the vessel completely. However, angiographic studies have identified culprit lesions that do not cause marked stenosis (31), and often an ACS occurs without the preceding symptoms of angina which would be indicative of a high grade stenosis.

Computed tomographic (CT) angiography, which showcases the arterial wall as well as the lumen, has revealed plaque characteristics associated with ACS events which include: low attenuation (i.e. little or no calcification) and an outward expansion of the artery wall which allows for very little encroachment on the lumen $(32,33)$. This expansion also accommodates the growth of the plaque for much of its development (34).

Instead of progressive narrowing of the lumen, it is the activation of vulnerable plaques that precipitates ischaemia and infarction. Some studies have noted the role of coronary spasm causing restricted blood flow to the myocardium, but in most cases the formation of an occluding thrombus on the surface of the plaque is the driver of infarction (35). Thrombosis can occur due to plaque rupture, which is detectable in 60 to $70 \%$ of cases, or less frequently due to endothelial erosion (36). Plaques vulnerable to rupturing, which most commonly cause a fatal ACS, often have a thin fibrous caps (37). This cap allows for a barrier between the immune and thrombogenic rich material of the necrotic core and the coagulation factors found in the blood. Thinning of this fibrous cap and degradation of collagen, which provides tensile strength, is caused by an excess of proteinases that break down collagen and an inhibition of smooth muscle cells to replenish it (38). These enzymes belong to the matrixmetalloproteinase (MMP) family and macrophages have been found to over produce all three of the human MMP collagenases (MMP-1, 8 and 13) in atherosclerotic 
plaques $(39,40)$. When the fibrous cap ruptures or is disrupted, blood is allowed to come in to contact with the thrombogenic core. Tissue factor, a potent pro-coagulant produced by macrophages, comes in to contact with the blood triggering thrombin generation and platelet activation and aggregation (40).

Superficial erosion of coronary plaques causes approximately $20-25 \%$ of deaths from an ACS (40). These lesions usually lack prominent inflammatory infiltrates and exhibit a much richer proteoglycan accumulation compared to rupture prone plaques. The mechanisms of superficial erosion have received much less attention than those involved in the rupture of the fibrous cap. Apoptosis of endothelial cells could contribute to desquamation and production of tissue factor (41). Oxidative stress, in particular the production of myeloperoxidase by activated leukocytes, can initiate endothelial cells to undergo programmed cell death. Endothelial cell loss and tissue factor production could propagate local thrombosis found in the coronary arteries (42).

Although it is noted in autopsy studies that frank rupture of plaques causes the majority of deaths (43), and superficial erosion making up the rest, there is growing evidence that not all plaque ruptures and thrombus formation cause coronary events (44). Rather, plaque rupture and thrombus formation occurs frequently at a subclinical level and is instrumental in plague progression, remodelling and later luminal narrowing $(34,45)$. Therefore, it is not only the act of plaque exposure to the blood, but also the combination of other factors and conditions with rupture or erosion that lead to the perfect storm resulting in an ACS (34). Tabled below is a list of factors and conditions associated with increased risk of ACS (Table 1-1). 
INTRODUCTION

Table 1-1 Factors and conditions associated with increased risk for an Acute Coronary Syndrome

\begin{tabular}{|c|c|c|c|c|c|}
\hline $\begin{array}{l}\text { Coronary Plaque } \\
\text { characteristics }\end{array}$ & $\begin{array}{l}\text { Coronary Blood } \\
\text { Flow Dynamics }\end{array}$ & $\begin{array}{l}\text { Intrinsic Hemostasis } \\
\text { Factors }\end{array}$ & $\begin{array}{l}\text { Metabolic and } \\
\text { Inflammatory Conditions }\end{array}$ & $\begin{array}{l}\text { Neurohormonal } \\
\text { imbalance }\end{array}$ & $\begin{array}{l}\text { Environmental } \\
\text { Factors and Drugs }\end{array}$ \\
\hline
\end{tabular}




\subsubsection{Treatment}

The management and treatment of ACS is dictated by where the patient falls on the ACS spectrum (Figure 1-2) and is intended to reduce myocardial necrosis and thus preserve left ventricular function and prevent major adverse cardiac events such as: ventricular fibrillation, decompensated heart failure and cardiogenic shock (46).

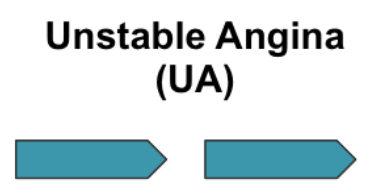

Angina pain with at least one of the following features:

- Is of new onset and severe

- Occurs at rest or with minimal exertion

- Pain is worsening in the severity and length of each episode (crescendo angina

- New ischaemic ST changes changes not including ST elevation

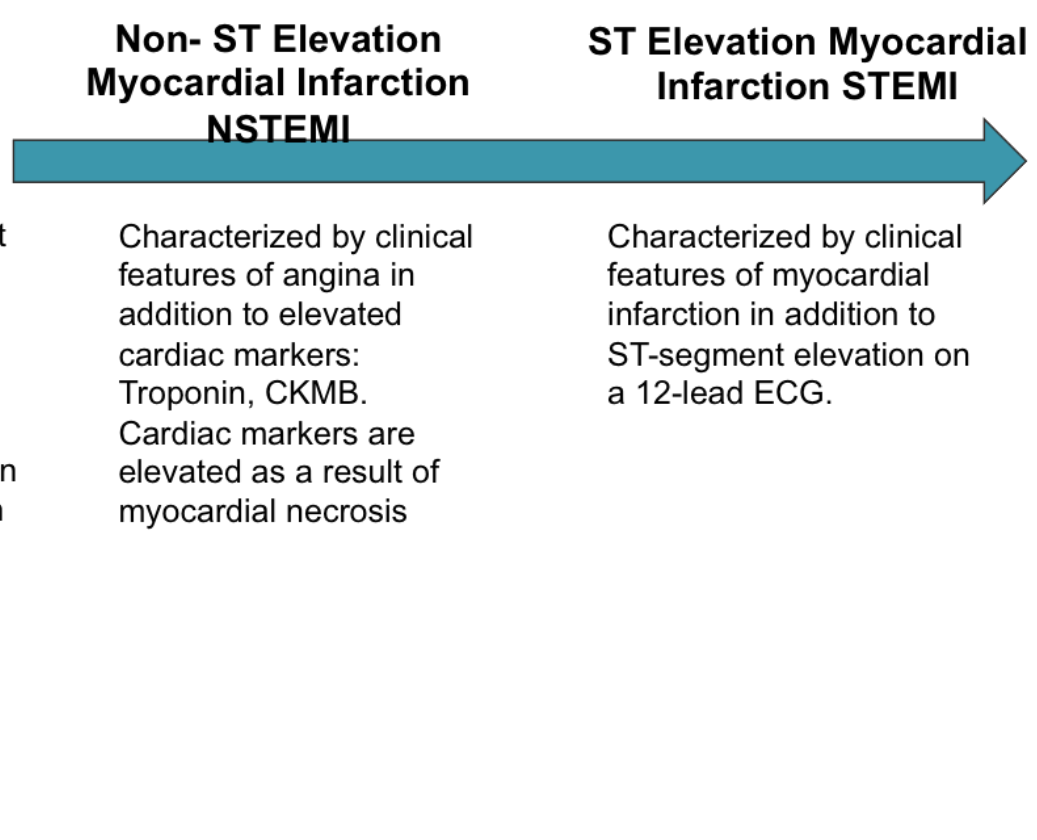

Figure 1-2 Spectrum of Acute Coronary Syndromes (ACS)

Adapted from C. Karras et al. Acute Coronary Syndromes. Disease-a-month (2013) 202-209 with permission from Elsevier.

Reperfusion of the ischaemic myocardium is the primary objective in the acute setting and restoring coronary flow to the muscle is time critical. Percutaneous coronary intervention $(\mathrm{PCl})$ with balloons or stents are the treatment of choice and are able to achieve restored coronary artery flow in $>90 \%$ of patients in optimal circumstances. Fibrinolytics are indicated in STEMI patients where a skilled facility and provider for primary $\mathrm{PCl}$ is not accessible within 90 minutes. Other adjunct therapies to manage the acute phase and chronic disease include: oxygen, nitroglycerin, analgesia, antiplatelet agents, hypertensive medications, anticoagulation and statin therapy. 


\subsubsection{Peripheral Vascular Disease}

Atherosclerosis as a systemic disease not only affects the coronary arteries but also the aorta, cerebral and lower arterial extremities. The manifestation of peripheral atherosclerotic disease has long been underestimated and under diagnosed, with large epidemiology studies showing a high prevalence of peripheral vascular disease (PVD) in the elderly, diabetics and patients with existing cardiovascular risk factors (47). Importantly, those with PVD have an increased risk of premature mortality and cardiovascular and cerebrovascular events (48-50). Studies using stress testing or coronary angiography found $60-90 \%$ of patients with PVD also had coronary disease and was an independent risk factor for cardiovascular death (50).

\subsubsection{Carotid Artery Disease}

Carotid artery disease is the formation and progression of atherosclerotic plaques in the carotid artery usually at the branching of the artery into its internal and external counterparts. The risk of cerebrovascular stroke is increased as the carotid artery becomes more stenosed with plaque but also as the morphology of the plaque becomes more unstable leading to a higher risk of embolism (51). Embolism from the carotid bifurcation is the most frequent pathogenetic mechanism of cerebral ischaemia (52). This ischaemia can be temporary causing Transient Ischaemic Attacks (TIA) or blood flow can be blocked permanently causing a cerebrovascular stroke and tissue death. TIAs can act as a warning sign as a plaque is becoming unstable and the risk of stroke is greatly increased in the short term (53). Cerebral ischaemia can cause paralysis, loss of vision, slurring of speech, numbness and loss of power on the contralateral side to the infarct.

Intervention with carotid endarterectomy surgery or carotid stenting in severe carotid stenosis $(>75 \%)$ is recommended to reduce secondary stroke risk particularly if the patient is symptomatic (54). Primary stroke prevention with surgery or stenting in asymptomatic patients with a severe stenosis is not as heavily recommended (55). Furthermore, as a significant proportion of cerebrovascular events occur in patients with carotid stenosis $<75 \%$ and as the embolic process is a significant driver of stroke, 


\section{INTRODUCTION}

therapies to reduce plaque progression and destabilistation such as antiplatelet, statins and anti-hypertensive drugs are also crucial (55).

\subsubsection{Lower Extremity Peripheral Vascular Disease}

Lower extremity peripheral vascular disease (PVD) is the atherosclerotic plaque obstruction of the arteries in the legs and pelvis. At the time patients are diagnosed with lower extremity PVD, $>50 \%$ present with asymptomatic disease or complain of atypical leg pain. A smaller percentage of patients who have lower extremity PVD also have intermittent claudication distinguished by cramping pain, discomfort, or a sensation of weakness in the legs that occurs with exertion and is relieved by rest. These symptoms are primarily attributed to the flow limiting stenosis or occlusion in the artery limiting oxygen supply to the muscle during exercise. Limb ischaemia also evokes an acute systemic and local response characterised by increased oxidative stress, inflammation and endothelial dysfunction (56-58).

Although claudication symptoms remain stable in $70 \%$ to $80 \%$ of patients, approximately $10 \%$ to $20 \%$ worsen and $1 \%$ to $2 \%$ of patients will progress to critical limb ischemia (CLI) over a 10-year period (59). Some patients undergoing amputations for $\mathrm{CLI}$ did not experience claudication pain 6 months previously, which indicates that not all PVD advances through the traditional classification schemes (60). In addition to functional impairment, as mentioned previously, PVD is a strong marker for coronary artery disease and increased risk of cardiovascular death. Interestingly, although the risk of death is higher in patients with PVD compared to those without, the risk is equal in patients who are symptomatic or asymptomatic (61).

Treatment of lower extremity PVD is intended to improve debilitating claudication symptoms and reduce cardiovascular morbidity and mortality. Along with revascularising the atherosclerotic arteries with surgery or percutaneous intervention where indicated, addressing risk factors such as smoking cessation, and proper diabetes and blood pressure management is also imperative for reducing symptom burden and cardiovascular risk. Studies have found the use of statins in lower 
extremity PVD can increase walking distance and slow progression of disease (62). Furthermore, the use of antiplatelet drugs in PVD with and without known coronary artery disease reduces the risk of stroke, $\mathrm{MI}$ and cardiovascular death $(63,64)$. Clear indications for revascularisation include ischemic rest pain, ischemic ulceration, gangrene, or symptomatic disabling claudication.

\subsubsection{Abdominal Aortic Aneurysm}

Patients with an abdominal aortic aneurysm (AAA) remain asymptomatic in most cases until rupture, which leads to death in $65 \%$ of patients (65). This highly fatal outcome is caused from the dilatation of the aortic wall and a weakening of the connective tissue to a point of rupture. As atherosclerotic lesions and thrombus are always present at the site of abdominal aneurysms, traditionally these aneurysms have been regarded as the same pathological consequence as coronary artery disease. However, this conventional view has been challenged by more recent studies showing a divergence from the athero-occlusive process with a more significant role of proteases pushing atherosclerotic plaques down an aneurysm path $(66,67)$. In particular the decreased expression of protease inhibitors such as, TIMP-2 and PAI-1, and increased production of MMPs, allow the imbalanced degradation of elastin and collagen in the aneurysm, which cross link with other connective molecules to provide strength and stability (68). The formation of a AAA is associated with a mural thrombus, which undergoes continual remodelling due to the constant blood flow in the aneurysm, unlike the turbulent flow in athero-occlusive disease (69). This mural thrombus has been implicated in reducing aneurysmal wall stress, but it's increasing thickness rich in platelets and thrombin can also lead to a local hypoxic environment in the aneurysm, exacerbating inflammation, apoptosis of smooth muscle cells and promoting remodelling of the arterial wall $(70,71)$.

The treatment of AAA with open surgery or endovascular stenting is based on the risk of rupture and is essentially prophylactic in nature, with the most benefit and risk reduction resulting from operating on aneurysms $>5 \mathrm{~cm}$ in diameter (69). As well as surgery, the proper management of cardiovascular risk factors is suggested, in 
particular smoking. There is a strong association between tobacco smoking and aneurysm development, with the prevalence of AAAs four times greater in smokers compared to non-smokers $(72,73)$. When relative risks were assessed in chronic smokers, the risk of developing a AAA is three-fold greater than developing coronary artery disease and five-fold higher than cerebrovascular disease (74). Therefore, smoking cessation is even more imperative in patients with AAA.

\subsection{Platelets}

\subsubsection{Function in Hemostasis}

Platelets are anuceleated cells in the blood, produced by megakaryocytes in the bone marrow and have been acknowledged for over 150 years for their important role in hemostasis and thrombosis (75). Platelets circulate at high shear rates and the primary physiological role is to sense damage to the endothelial wall and plug any circulatory leak. Platelets are activated by exposed collagen binding to the glycoprotein VI (GPVI) on the platelet at the site of injury, allowing the platelet to adhere to the substratum. Firm adhesion promotes the release of intracellular stores from granules, including adenosine diphosphate (ADP) and thromboxane $A 2$ (TXA2), which triggers the recruitment and activation of more platelets. During activation platelets undergo dramatic shape change which is calcium dependent and form into spiny spheres with finger like projections using their extensive actin cytoskeleton (76). In addition to shape change and granule release, activation leads to the important conformational change of the integrin glycoprotein IIb/IIla (GPIlb/IIla) increasing its affinity for fibrinogen, von Willebrand factor (VWF) and fibronectin to promote firm plateletplatelet adhesions (77). Activated platelets produce thrombin, which triggers the coagulation cascade resulting in the formation of a fibrin mesh encapsulating crosslinked platelets, red blood cells and leukocytes that effectively plugs the hemostatic leak. 


\subsubsection{Platelets in Atherosclerosis}

In addition to hemostasis, platelets also contribute to pathological processes such as host defence from microbial infection, sepsis, autoimmune diseases, tumour growth and metastases and atherosclerotic plaque progression, rupture and thrombosis (75).

\subsubsection{Plaque initiation and development}

Platelets are not only activated by collagen, but by an array of extra cellular matrix proteins and soluble factors in the blood such as ox-LDL, thrombin, ADP, serotonin and shear stress (78). Endothelial dysfunction and injury exposing pro-coagulant proteins to the circulation, as well as pro-inflammatory states such as diabetes; results in the increase of activated circulating platelets. Upon activation, platelets up-regulate several ligands that allow the cell to interact with the endothelium and leukocytes, promoting the initial inflammatory infiltration in atherosclerosis and progression of plaques. The platelet glycoproteins GPIba, GPIIb/IIla and GPVI have important roles in platelet-endothelial interactions (79-81) and GPIba and GPVI have shown to mediate platelet-leukocyte interactions $(82,83)$. Platelets are able to tether to atherosclerotic endothelium and bind to leukocytes under high vascular shear to aid in leukocyte infiltration (84). Inhibition or knock out of these ligands in animal models has resulted in the reduction of plaque progression $(83,85)$ and interestingly, GPVI has been proposed as a novel diagnostic marker for ACS in clinical studies (86).

P-selectin is one of the primary ligands for platelets' involvement in atherosclerosis, highlighted from P-selectin-deficient animal studies showing protection from the disease (87). It is expressed on platelets and is also stored and released in a soluble form from $\alpha$-granules. Platelets bind to the endothelium through P-selectin, but also to leukocytes via P-selectin glycoprotein ligand 1 (PSGL-1). Through this communication, multicellular aggregates can form which promotes the release of chemokines CCL2, CCL5 and cytokines like interleukin (IL)-1 $1 \beta$ to further activate and recruit leukocytes and promote atherosclerosis $(88,89)$. Platelet-Leukocyte aggregates are able to tether and roll on endothelial cells with a higher avidity than unconjugated leukocytes, enhancing endothelial activation and leukocyte 


\section{INTRODUCTION}

transmigration (90). Soluble (s)P-Selectin has shown to be a good biomarker of platelet and/or endothelial cell activation and high levels indicate an elevated risk for future cardiovascular events independent of traditional risk factors (91). Beyond a biomarker function, sP-Selectin is also considered an active player in inflammation during atherosclerosis, potentially through binding to PSGL-1 on leukocytes and promoting pro-coagulant microparticle generation via the Mac-1 pathway (92).

CD40 ligand (CD40L), a member of the TNF-family, is one of the best characterised costimulatory molecules that enhances immune responses and inflammation through binding to CD40 and inducing cell activation and differentiation $(93,94)$. Platelets express CD40L on activation and can also shed the ligand, contributing to the soluble CD40L in the circulation. The presence of platelet bound and soluble CD4OL enable binding of CD40 on the endothelium and different leukocyte subsets. Atherosclerotic lesions are abundant in CD40L expressing cells and platelet expressed CD40L represents the majority of circulating CD4OL (95). Studies investigating the inhibiton of the CD40-CD40L stimulation found a reduction in atherosclerotic plaque size and an increase in stable fibrous lesions (95). Through CD4OL, platelets can bind to lymphocytes, particularly $\mathrm{T}$ cells and promote pro-inflammatory effector cell generation and reduce Treg populations $(96,97)$. Soluble CD40L has been demonstrated to be a potential biomarker for atherosclerotic instability, with higher levels found in patients experiencing acute atherosclerotic infarctions and patients with atherosclerotic disease compared to healthy controls (98). Additionally, platelets express scavenger, chemokine and toll-like receptors that also have key roles in atherosclerosis development and have been reviewed by several groups (98-100)

Platelets also release a multitude of chemokines, cytokines and other immune modulatory proteins that drive atherosclerosis. The chemokines Platelet Factor 4 (PF4 or CXCL4), RANTES (CCL5), CDCL7 and stromal-cell derived factor-1 (SDF-1 or CXCL12) are stored in $\alpha$-granules and released on activation. In particular RANTES and PF4 have been studied extensively and are both pro-atherogenic and act to selectively recruit monocytes, neutrophils and T cells to injured endothelium (101-104). The cytokine IL-1 $\beta$ is recognized as a main driver of platelet-induced endothelial cell 
activation (105). Release of this cytokine causes endothelial secretion of IL-6, IL-8, CCL2 and expression of adhesion molecules ICAM-1 and $\alpha v \beta 3(106,107)$. Transforming growth factor $\beta$ (TGF- $\beta$ ) is a potent modulator of the immune system and promotes anti-inflammatory and wound healing responses. Interestingly, platelets are the largest containers of TGF- $\beta$ in the body and release it in abundance on activation/degranulation (75). The role of platelet released TGF- $\beta$ remains unclear, with potential roles in promoting Treg development to counter pro-inflammatory responses in the plaque but also proatherogenic functions in promoting calcification and apoptosis (108). Platelet derived TGF- $\beta$ may also provide key signals during thrombosis to induce matrix production (109).

There are multiple other expressed receptors and secreted mediators that platelets produce to influence plaque initiation and development as reviewed by Lievens and von Hundelshausen (78). Overall the platelet has a big impact on determining the inflammatory milieu of the plaque and the progression from stable to unstable potentially resulting in a thrombotic event as summarised in Figure 1-3. 


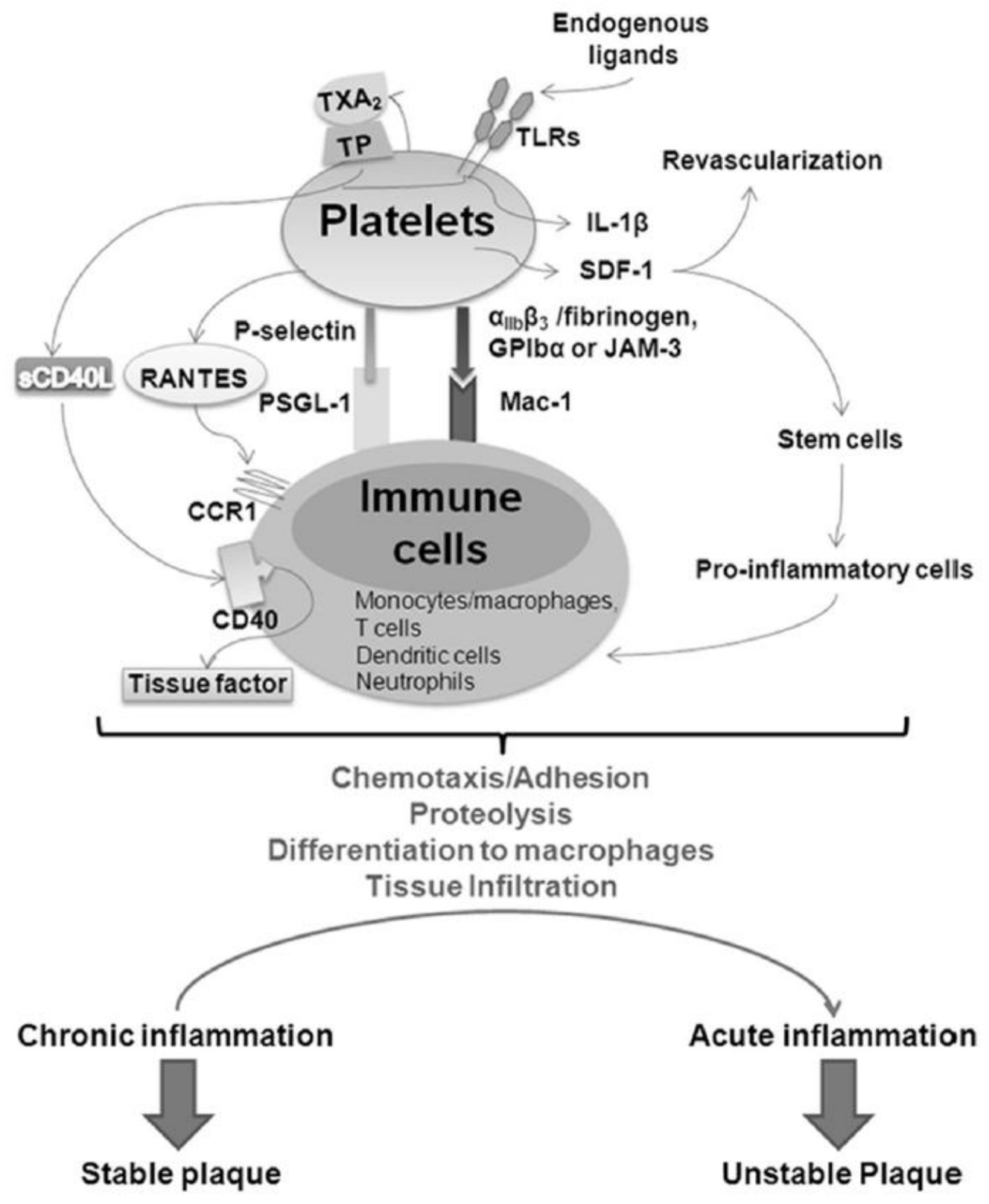

Figure 1-3 Role of platelets in the vascular inflammatory response associated with atherosclerosis

Platelets interact with immune cells via both cell to cell contact and platelet derived soluble mediators. These interactions can lead to increased levels of chemotaxis and adhesion in immune cells as well as differentiation and infiltration. This heightened inflammation can lead to the phenotype of plaque to change from stable to and unstable phenotype. CCR1, Chemokine (CC motif) receptor 1; JAM-3, Junctional adhesion molecule 3; TXA 2, Thromboxane A2; TP, Thromboxane A2 receptor; TLRs, Toll-like receptors; IL-1_, Interleukin-1 beta; SDF-1, Stroma-cell derived factor-1; GPIb_, glycoprotein Ib_; PSGL-1, P-Selectin Glycoprotein Ligand 1; and Mac-1, Macrophage 1 antigen. Lievens $D$ and Von Hudelshausen P. Platelets in atherosclerosis. Thrombosis Haemostasis 2011; 106: 827 - 838. Permission for reproduction sough from Elsevier. 


\subsubsection{Platelets in thrombosis}

The main role of platelets in the body is thought to be clot formation and therefore it is not surprising that in atherosclerosis, platelets drive the late events of thrombus formation during plaque rupture, microembolism, or spasm within the microcirculation. The mechanisms involved in hemostasis and thrombosis are similar with early key roles for exposed collagen coupled with VWF and GPIV to initiate adhesion of platelets to injured endothelium and exposed plaque (111).

The propagation of thrombus is reliant on soluble agonists such as ADP, thromboxane and $\alpha$-thrombin, as only the first layer of platelets are in direct contact with thrombogenic proteins. These soluble agonists induce a broad range of biochemical and functional platelet responses-including intracellular $\mathrm{Ca}^{2+}$ flux, platelet shape change, and the secretion of granule contents-coincident with the formation of platelet aggregates (112). However, recent studies have shown discoid shaped platelets adhering to growing thrombi in the early stages that do not undergo shape change, $\mathrm{Ca}^{2+}$ or $\alpha$ granule release, indicating that additional platelet aggregation mechanisms could be involved $(112,113)$. One of these is the biomechanical mechanism of activation via shear stress due to arterial blood flow promoting thrombus growth (114).

After platelets activate and aggregate at the sites of vascular lesions, the thrombus undergoes stabilisation to maintain its location against opposing blood flow. Newly recruited platelets to a growing thrombus send feedback signals to platelets in the deeper layers of the thrombus to prevent disaggregation. This leads to strengthening of the interactions between adhesive ligands (VWF, fibrinogen, fibronectin) and receptors (GPIba and GPIIb/IIla). In particular, ADP signaling through the P2Y1 and P2Y12 receptors causing cyclic $\mathrm{Ca}^{2+}$ oscillations is vital to maintain GPIIb/IIla activation, which provides the thrombus stability in flowing blood. Brass et al present an exhaustive review on this feedback system in thrombus stabilization (116).

Thrombosis is also a potentially life threatening complication after the implantation of stents or grafts in atherosclerotic lesions. Stent thrombosis (ST) is a relatively 
uncommon complication but it's mortality and morbidity is high with $20-40 \%$ of cases causing death and $50-70 \%$ causing myocardial infarction and the need for revascularisation $(116,117)$. The occurrence of stent thrombosis is directly related to the acuity of the clinical syndrome before stenting. A patient presenting with an ACS has a several fold increase risk of stent thrombosis independent of stent type compared to a patient with stable coronary symptoms $(118,119)$. This risk is related to underlying inflammation and unstable plaque at the time of implantation leading to incomplete endothelialisation, less neointima formation allowing for continued exposure of circulating platelets to the injured vessel (120). Patients with critical limb ischaemia undergoing femoral bypass surgery and endovascular procedures are at high risks of graft occlusion and limb amputation (122). The risk of limb amputation and death, also increases significantly with every revascularisation procedure (123).

\subsection{Antiplatelet agents and Non-Response}

Platelets are the drivers of thrombotic events in atherosclerosis and are central to disease progression. Therefore, antiplatelet therapy is a cornerstone of the pharmacologic treatment strategy in patients with cardiovascular disease. As platelet activation and aggregation is controlled by complex and overlapping pathways, there are many targets for antiplatelet drugs to prevent ischaemic events as displayed in Figure 1-4. 


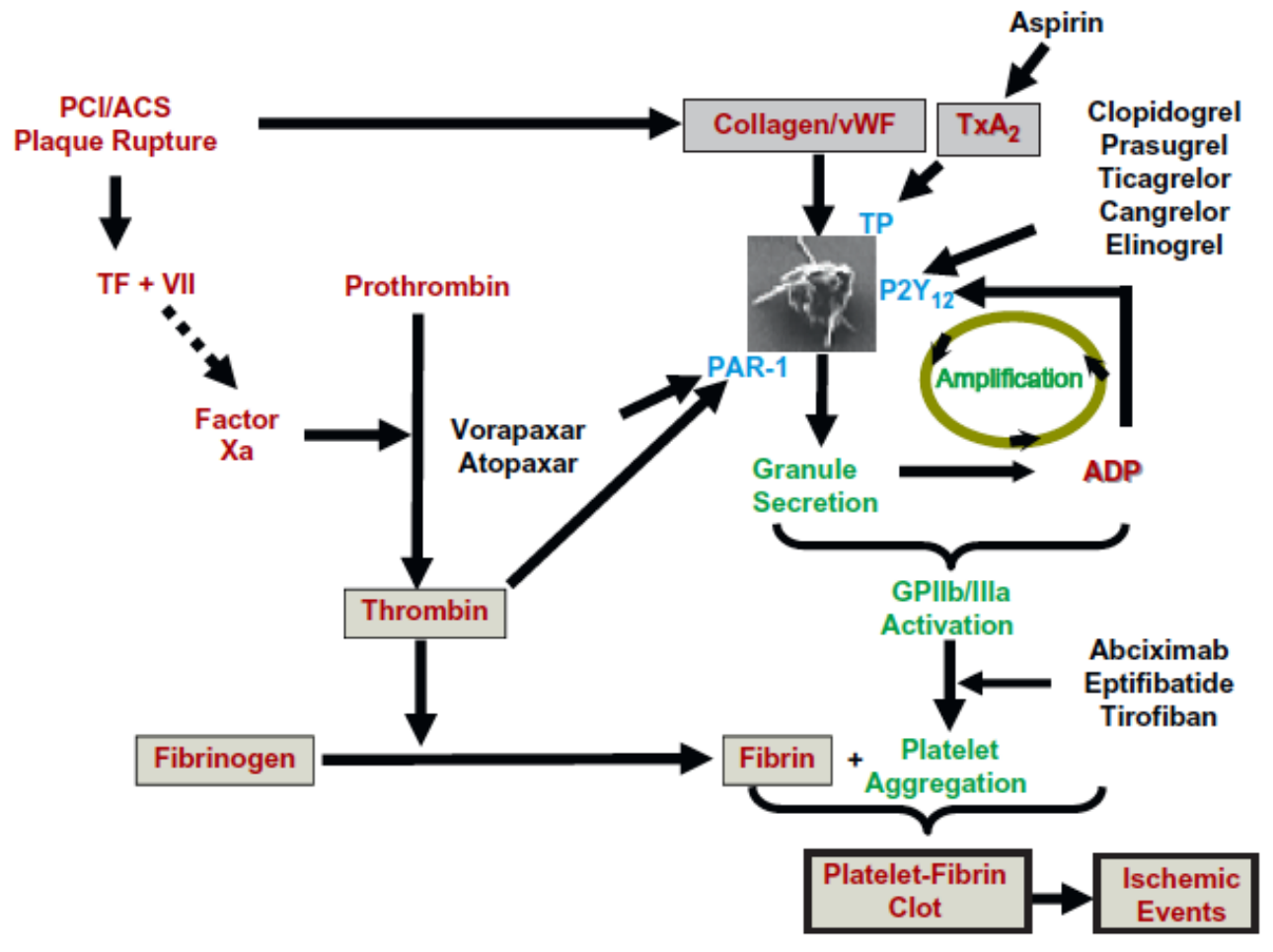

Figure 1-4 Antiplatelet agent targets to prevent ischaemic events

The process of $\mathrm{PCl}$ or plaque rupture leads to an increase in the release of TF, initiating the coagulation cascade and the release of collagen, activating platelets. Platelet activation is a complex process involving many receptors and signalling pathways highlighted in blue. Platelet activation leads to several secondary pathways including granule secretion and GPIlb/llla as highlighted in green. Therefore, antiplatelet drugs highlighted in black, target multiple pathways to inhibit platelet activation and aggregation to prevent ischaemic events. Gurbel PA and Tantry US, Monitoring of antiplatelet therapy, Platelet Third Edition, 2013; 603-633. Reproduced with permission from Elsevier. ACS, acute coronary syndromes; ADP, adenosine diphosphate; PAR-1, pro-tease activated receptor1; GPIlb/llla, glycoproteinllb/llla; PCl, percutaneous coronary intervention; TF, tissue factor; TP, thromboxane receptor; TXA2, thromboxaneA2; vWF, von Willebrand factor.

The use of dual antiplatelet therapy (DAPT) comprising of aspirin and a P2Y12 inhibitor, commonly clopidogrel, is the most widely used combination for prevention of ischaemic events in ACS patients (123). A summary of the current European and American guidelines for antiplatelet therapies is shown in Table 1-2. The widespread use of these agents, coupled with more evidence-based interventions and therapies, has led to a significant reduction in adverse events following an ACS, including death, over the last decade (125). However, adverse events still occur in these patients who are on DAPT and recent studies have observed that there is a wide inter-individual variability in the response to therapy. A proposed therapeutic window exists where a patient with high on treatment platelet reactivity (HOTPR) is at risk of ischaemic events and a patient with low on treatment platelet reactivity (LOWPR) is at risk of 
bleeding complications. The use of aspirin and clopidogrel in atherosclerosis as well as the prevalence of HOTPR and LOWPR and their contributing risk factors are summarized below. 
INTRODUCTION

Table 1-2 Guidelines for Antiplatelet therapy in patients with CAD

\begin{tabular}{|c|c|c|c|c|c|c|c|}
\hline \multirow[t]{2}{*}{ 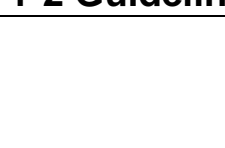 } & \multirow{2}{*}{$\begin{array}{l}\text { Preclinical } \\
\text { CAD } \\
\text { ESC (125) }\end{array}$} & \multirow{2}{*}{$\begin{array}{l}\text { Stable } \\
\text { CAD } \\
\text { ESC (125) }\end{array}$} & \multirow[t]{2}{*}{ Elective PCI } & \multicolumn{2}{|l|}{ UA/NSTEMI } & \multicolumn{2}{|l|}{ STEMI } \\
\hline & & & & ESC $(123,125)$ & AHA/ACC (126) & ESC (125) & $\mathrm{AHA} / \mathrm{ACC}(128)$ \\
\hline Aspirin & II-A & I-A & I-A (125) & I-A & $\mathrm{I}-\mathrm{A}$ & $\mathrm{I}-\mathrm{A}$ & I-A \\
\hline Clopidogrel & & I-A & I-A (125) & $1-B^{a}$ & $I-B^{b}, I-A^{c}$ & $\mathrm{I}-\mathrm{C}$ & $\mathrm{I}-\mathrm{C}$ \\
\hline Prasugrel & & & & Ila-B, I-B ${ }^{d}$ & I-B & I-B & I-B \\
\hline Ticagrelor & & & & I-B & $f$ & I-B & $f$ \\
\hline
\end{tabular}

aOnly if prasugrel or ticagrelor are not an option. ${ }^{\mathrm{b}}$ Before $\mathrm{PCl}$, ${ }^{\mathrm{c} A t}$ the time of $\mathrm{PCl}$. d In case of known coronary anatomy, intent to $\mathrm{PCl}$ and no pretreatment with $\mathrm{P} 2 \mathrm{Y} 12$-inhibitiors, ${ }^{\mathrm{e}} \mathrm{At}$ the time of $\mathrm{PCl}$, if not pretreated with thienopyridines, ${ }^{\mathrm{f}} \mathrm{Not}$ approved at the time of guidelines compliation.

ACC, American College of Cardiology; AHA, American Heart Association; CAD, coronary artery disease; ESC, European Society of Cardiology; NSTEMI nonST segment elevation myocardial infarction; PCl, percutaneous coronary intervention; STEMI, ST elevation myocardial infarction

Classes of Recommendation:

Class I: Benefits >>> Risk: Procedure/ treatment SHOULD be performed/administered.

Class Ila: Benefits >> Risk: Additional studies with focused objectives needed; it is REASONABLE to perform procedure/administer treatment.

Levels of Evidence:

Level A: Multiple populations evaluated; data derived from multiple randomized clinical trials or meta-analyses

Level B: Limited populations evaluated; data derived from a single randomized trial or nonrandomized studies

Level C: Very limited populations evaluated; only consensus opinion of experts, case studies, or standard of care 


\section{INTRODUCTION}

Clinical trials investigating the use of DAPT in patients with PVD is limited. The only available evidence comes from a substudy of the large DAPT trial CHARISMA, which evaluated the effect of aspirin plus clopidogrel versus aspirin alone in patients with established and at risk for cardiovascular disease (128). In a subgroup analysis of PVD patients, there was no observed benefit in the DAPT arm at reducing cardiovascular events compared to aspirin monotherapy (130). Further trials in larger PVD populations are needed to investigate whether DAPT therapy can reduce risk over standard aspirin therapy.

\subsubsection{Aspirin therapy}

Aspirin blocks thromboxane $A_{2}\left(T X A_{2}\right)$ mediated platelet aggregation through the acetylation of the COX-1 enzyme (Figure 1-5) and this process is irreversible as platelets are enucleate and are unable to resynthesize COX-1 (130). However, as aspirin is not a direct inhibitor of thromboxane, it has been demonstrated that even a $10 \%$ residual thromboxane synthase activity is sufficient to cause aggregation (132). Furthermore, it is believed a $95 \%$ inhibition of thromboxane is needed to observe clinical efficacy from aspirin therapy (133). COX-2, even at low levels is also able to induce platelet aggregation via thromboxane A2 and aspirin inhibits COX-2 but 166 times less effective than COX-1. Additionally, endothelial cells and monocytes/macrophages can rapidly (2-4 hours) recover from the aspirin effect by resynthesizing the COX-2 enzyme and may contribute prostaglandin $\mathrm{H} 2$ to platelets that synthesize TXA2 by thromboxane synthase (transcellular TXA2 synthesis), as detailed in Figure 1-5. As atherosclerotic plaques have an abundance of monocytets/macrophages, this local provider of COX-2 may be an important mechanism that platelets use to overcome the antithrombotic effect of aspirin therapy. Under normal conditions, COX-2 only comprises $10 \%$ of platelet COX activity. However, in environments such as post coronary artery bypass (CABG) surgery or diabetes, where there is high turnover and a higher proportion of young platelets, COX- 2 activity can be as high as $60 \%$ and may contribute to thromboxane production despite COX-1 inhibition (133). 


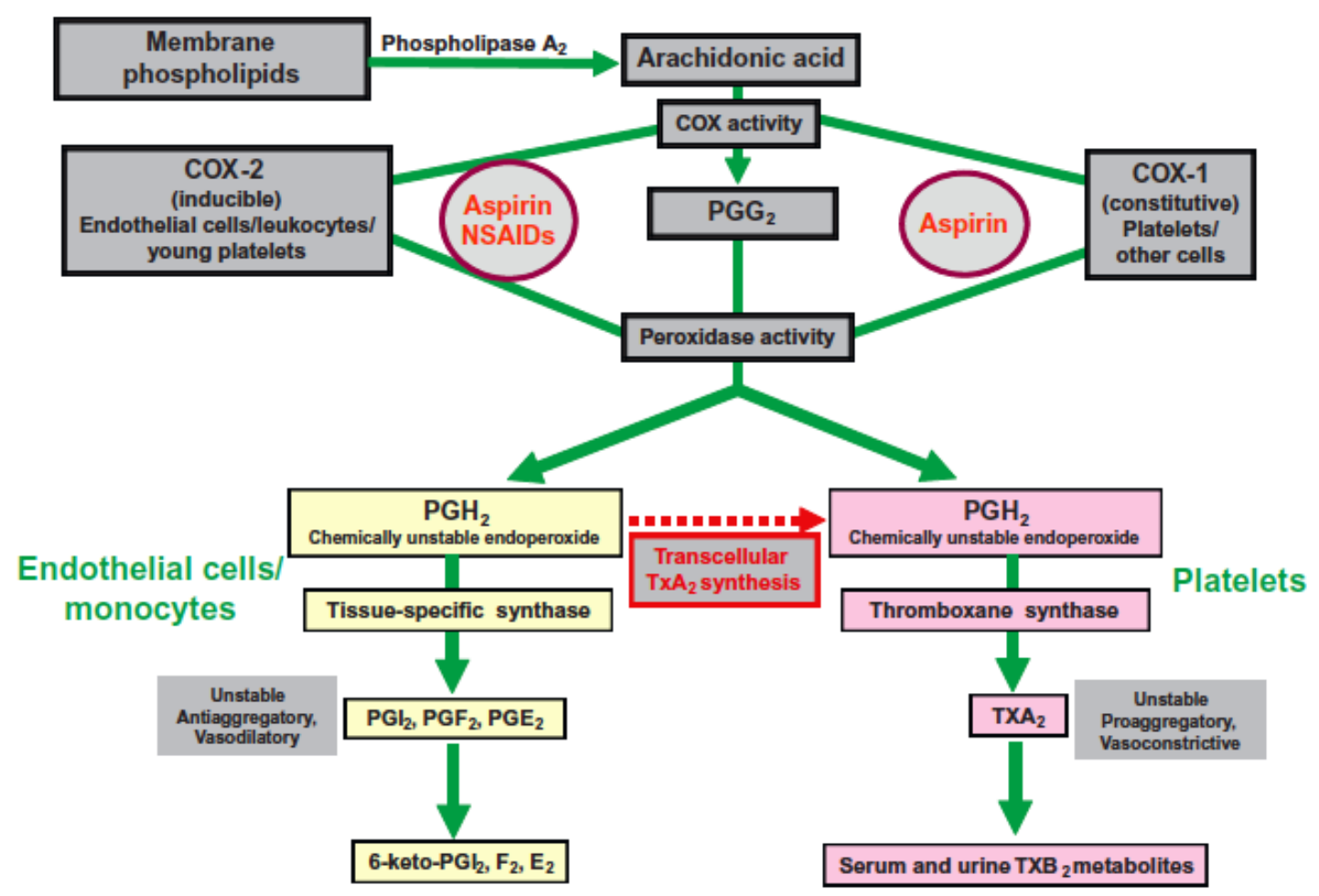

Figure 1-5 Aspirin effects via the inhibition of COX activity.

Aspirin has inhibitory effects on both COX-1 and COX-2 activity. Aspirin prevents the access of arachidonic acid to the catalytic site of COX-1. In platelets (highlighted in pink) this leads to a reduction thromboxane $\mathrm{A} 2$ production, which promotes aggregation and this inhibition is irreversible. However, in endothelial cells and monocytes (highlighted in yellow), the aspirin effect can be overcome by resynthesising the COX-2 enzyme and may contribute $\mathrm{PGH}_{2}$ to platelets by Transcellular $\mathrm{TxA}_{2}$ synthase. COX, cyclooxygenase; TX, thromboxane; PG, prostaglandin; NSAIDs, nonsteroidal antiinflammatory drugs Gurbel PA and Tantry US, Monitoring of antiplatelet therapy, Platelet Third Edition, 2013; 603-633. Reproduced with permission from Elsevier.

Aspirin remains the drug of choice for secondary prevention of recurrent ischaemic events in ACS patients (130). Conversely, aspirins' role in primary prevention of ACS remains unclear and somewhat controversial $(134,135)$. In the setting of established preclinical vascular disease without the presence of other risk factors, aspirin has a Class II-A indication for use but the risk of bleeding must be taken in to consideration (134). There is also no apparent benefit of daily doses above $100 \mathrm{mg}$ from metaanalyses and a negative dose-dependant effect on gastrointestinal complications with increasing doses $(136,137)$. It is hoped that continuing studies such as ASCEND (NCT00135226) and ACCEPT-D (ISRCTN48110081), both investigating aspirin for primary cardiovascular event protection will provide clarifying information. 


\section{INTRODUCTION}

Early administration of aspirin in ACS has consistently been shown to be beneficial over all factions of ACS including: unstable angina/NSTEMI $(138,139)$ and STEMI $(140)$. Aspirin HOTPR has been extensively reviewed and several studies have linked the presence of HOTPR to a higher risk of recurrent ischaemic events $(141,142)$. A systematic review and meta-analysis of various studies linking aspirin HOTPR to clinical outcomes utilising different methods, an odds ratio of all cardiovascular outcomes of 3.8 (95\% Cl; 2.3-6.1) for aspirin HOTPR was observed (143). Additionally, the important role of aspirin in ACS is underlined by the nearly two-fold (OR 1.82, 1.522.18, $\mathrm{p}<0.00001$ ) increased risk of adverse events in patients with an ACS or history of CAD who discontinued or were not compliant with their aspirin therapy (145).

Despite the abundance of data demonstrating the efficacy of aspirin therapy in patients with CAD, the data in PVD is less compelling. Aspirin therapy has been demonstrated to decrease cardiovascular events in patients with PVD, but has little or no effect on symptomatic improvement in subjects with intermittent claudication (146). In a recent meta-analysis of 18 prospective randomised studies including 5269 patients with PVD, aspirin resulted in $12 \%$ reduction of the combined end point of nonfatal $\mathrm{MI}$, nonfatal CVA and cardiovascular death but failed to reach statistical significance (64). Whether studies with a larger number of vascular patients would provide more convincing benefit is unknown. Because a large majority of PVD patients also have symptomatic CAD or cerebrovascular disease, aspirin is an acceptable antiplatelet agent in this setting and the ACCF/AHA endorse aspirin with class I, level of evidence B recommendation (146). Further studies are needed to elucidate the relationship between aspirin HOTPR and risk in PVD patients.

\subsubsection{Aspirin HOTPR}

Estimates of the prevalence of aspirin HOTPR vary dramatically (from 5.5\% to 56.8\%), depending on the method of platelet function testing, cut-point used and the population (148). Aspirin HOTPR has also been linked to high platelet reactivity in the response to other agonists such as ADP and collagen and may reflect a global hyperreactivity (148). The concomitant use of particular NSAIDs may influence the response 
to aspirin by interfering with aspirins ability to bind COX-1 (150) and the use of these drugs has been associated with an increased risk of cardiovascular events (150). Genetic polymorphisms in the genes encoding COX-1, COX-2, GPIIIa, P2 $\mathrm{Y}_{12}$, and P2 $\mathrm{Y}_{1}$ have been suggested and studied for their potential influence on aspirin response. $A$ systematic review looking at 50 studies investigating 9 genes in more than 11,000 people only found a significant relationship between the PLA1/A2 polymorphism in the GPIIla receptor and an increased rate of aspirin HOTPR in healthy subjects but not CAD patients (151). The relationship between gene polymorphisms and aspirin responsiveness remains unclear.

When aspirin HOTPR is identified in patients, the treatment to correct this is still unknown. Currently, there is no published study that has investigated the clinical efficacy of changing therapy based on aspirin response and therefore guidelines do not recommend routine testing of aspirin response or tailoring of therapy based on these tests (152). Furthermore, there are only a handful of studies that have investigated aspirin HOTPR in a strictly PVD population and have found mixed findings between HOTPR and adverse events $(153,154)$.

\subsubsection{P2Y12 Inhibitors}

P2Y12 inhibitors are used in conjunction with aspirin to prevent secondary ischaemic events in patients with ACS. The drugs work by blocking the ADP platelet amplification pathway by antagonising P2Y12 receptors on platelets and inhibiting GPIIb/IIla formation. The first P2Y12 inhibitor Ticlodopine, belonging to the Thienopyridine family of drugs, was discovered after antiplatelet/aggregation screening studies in the 1970s. Ticlodopine was found to have greater antithrombotic efficacy than aspirin in many clinical settings including; secondary protection against ischaemic events following stroke, TIAs and unstable angina, leading to FDA approval in 1991 (156). A further 5 years later, a study published in the New England Journal of Medicine found DAPT therapy of aspirin plus ticlodopine decreased the risk of $\mathrm{MI}$ and repeat revascularisation after $\mathrm{PCl}$ by $82 \%$ compared to aspirin alone (156). However, ticlodopine had unfavourable side effects such as diarrhoea, nausea and vomiting in 
up to $50 \%$ of treated patients (157). But some patients also suffered from rare but serious side effects including neutropenia and thrombotic thrombocytopenic purpura, which lead to the recommendation of regular full blood count tests in the first 3 months of treatment (158). The development of clopidogrel, another theinopyradine with a similar efficacy for preventing vascular events, but a much more favourable safety profile (160), lead to clopidogrel becoming the most commonly prescribed drug in the world (160).

\subsubsection{Clopidogrel}

Clopidogrel is a pro-drug that requires hepatic biotransformation into its active metabolite to exert its antiplatelet response. Only $10-15 \%$ of the absorbed drug undergoes biotransformation, the remaining $85-90 \%$ is hydrolysed by hepatic carboxylase to an inactive carboxylic acid metabolite. The metabolism of clopidogrel is a two-step process controlled by hepatic cytochrome (CYP) P450 isoenzymes (Figure 1-6). The first step involves the oxidation of the thienol ring to 2-oxo-clopidogrel and studies have indicated the CYP isoenzymes CYP2C19, CYP1A2, and CYP2B6 as the main enzymes involved. The second metabolism step produces the highly unstable active metabolite (R-130964) through hydrolysis and similarly to the first step, CYP2C19 is actively involved in the metabolism along with CYP2C9, CYP2B6, and CYP3A (161). The active metabolite of clopidogrel covalently and specifically binds to the P2Y12 receptor on platelets trafficking through the hepatic circulation. This interaction is non-reversible and blocks ADP induced platelet aggregation for the platelets lifetime. 
<smiles>COC(=O)[C@H](c1ccccc1Cl)N1CCc2sccc2C1</smiles>

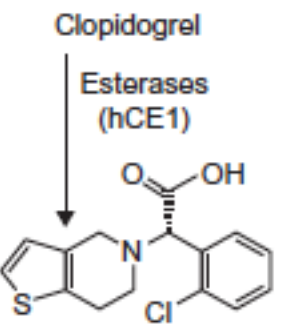

Clopidogrel inactive acid metabolite

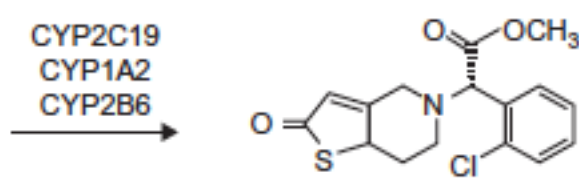

2-Oxo-clopidogrel

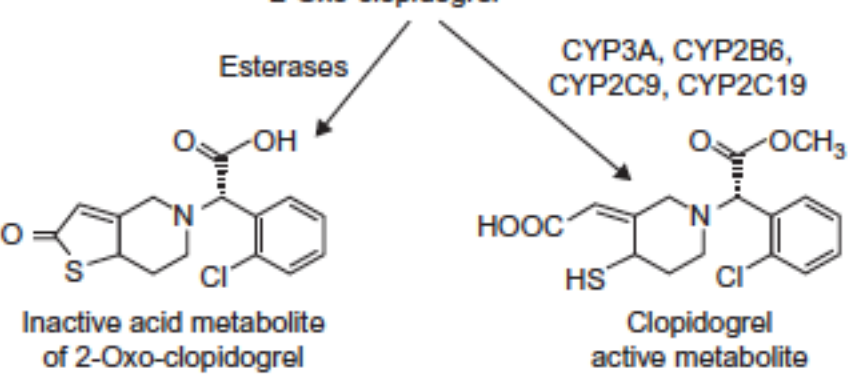

Figure 1-6 Metabolism of clopidogrel

Cattaneo M, ADP Receptor Antagonists, Platelet Third Edition, 2013; 1117-1138. Reproduced with permission from Elsevier.

Clopidogrel has shown to be very effective in secondary prevention of ischaemic events in patients with established cardiovascular disease (63). However, clopidogrel as a single or dual antiplatelet therapy is not indicated for primary prevention of ischaemic events in patients with preclinical cardiovascular disease or multiple risk factors. In the primary prevention cohort of the CHARISMA trial, there was no clear benefit of DAPT with clopidogrel over aspirin alone and subgroup analysis revealed a higher mortality and bleeding risk in patients on DAPT who were asymptomatic (162, 163). This cohort also comprised a large proportion (42\%) of diabetics and currently there is no evidence to support DAPT in this high-risk group for primary prevention.

The CAPRIE study investigated the risk of recurrent ischaemic events in a larger cohort $(19,185)$ of high-risk patients due to a previous ACS, ischaemic stroke or peripheral vascular disease (63). The study found clopidogrel single therapy (75mg) to be superior to aspirin therapy $(325 \mathrm{mg}$ ) at preventing major cardiovascular adverse events with an $8.7 \%$ relative risk reduction of $\mathrm{MI}$, ischaemic stroke and vascular death in those treated with clopidogrel. The use of DAPT (aspirin and clopidogrel) in stable cardiovascular disease has not been directly tested in a prospective randomised trial. However, post-hoc analysis from the CHARISMA study in patients with a history of 


\section{INTRODUCTION}

cardiovascular events (similar to CAPRIE) showed an improvement in prognosis without excess bleeding in those who were on DAPT compared to aspirin alone (128). Interestingly, the reduced risk of $\mathrm{MI}$, stroke and cardiovascular death seen in stable coronary disease patients on DAPT was also found in the peripheral vascular disease subgroup (164). In the context of cerebrovascular ischaemia and secondary prevention of TIAs or addition strokes, clopidogrel has some data to support its use over aspirin although the size of the additional benefit is controversial (165). The use of DAPT in preventing recurrent stroke and TIAs is not favoured over aspirin or clopidogrel alone. The MATCH trial, a study of high-risk patients with recent ischaemic stroke or TIA and at least one risk factor, found DAPT compared to clopidogrel alone resulted in a non-significant reduction in major vascular events but a doubled relative risk in life threatening hemorrhages (166). Although analyses of predefined subgroups in the MATCH trial did not identify patient groups where DAPT therapy was advantageous and bleeding risk was relative to single clopidogrel therapy.

In contrast to preclinical and stable cardiovascular disease, there is a wealth of trial data supporting the use of DAPT in patients following an ACS or PCI. The sustained and incremental benefit of clopidogrel on top of standard therapy, including aspirin, was demonstrated in the CURE trial in unstable angina (UA), non-ST elevation MI (NSTEMI) (167) and acute ST-elevation MI patients (STEMI) (168). The UA and NSTEMI patient cohort received clopidogrel $(300 \mathrm{mg})$ within 24 hours of symptom onset and chronically (75mg) on top of standard therapy for 3-12 months following the event. This lead to a $20 \%$ risk reduction of MACE in patients receiving clopidogrel compared to the placebo group. With a more potent antiplatelet added on top of standard therapy, not surprisingly the rate of major bleeding was greater in the clopidogrel group compared to placebo ( $3.7 \%$ vs $2.7 \%, p<0.001)$, although the rates of life threatening bleeds and hemorrhagic stokes were similar (2.1\% vs $1.8 \% \mathrm{p}=0.3)$ (167). In the STEMI patient group a loading dose of clopidogrel $(300 \mathrm{mg})$ was given within 12 hours of ACS onset and compared to standard therapy. Patients who received clopidogrel had a $20 \%$ risk reduction in MACE at 30 days. Furthermore, the addition of clopidogrel in the acute setting lead to $36 \%$ reduced risk of an occluded infarctrelated artery on angiography, which reduces reperfusion and increases complication 
risk, and decreased rates of recurrent $\mathrm{MI}$ and death before angiography could occur (168). The COMMIT trial randomly allocated clopidogrel $(75 \mathrm{mg})$ or placebo to a large cohort of patients $(45,852)$ with suspected MI on top of daily aspirin. Although treatment was of a short duration (average 15 days); clopidogrel treatment was again associated with a statistically significant reduction in the incidence of death, reinfarction and stroke, and was consistent over a wide range of patients and independent of other therapies used (169).

Coronary revascularisation with the use of coronary balloons or stents in both elective and acute settings is associated with an increase in thrombosis risk and therefore adequate antiplatelet therapy is even more critical after $\mathrm{PCl}$. Within the CURE trial, a pre-specified subset who underwent PCI (PCI-CURE) were assessed to investigate the benefit of a pre-procedural loading dose of clopidogrel $(300 \mathrm{mg})$ followed by long term daily doses $(75 \mathrm{mg})$. A 31\% reduction in cardiovascular death and MI risk was observed in the patients receiving clopidogrel and a 30\% risk reduction in target-vessel revascularisation at 30 days compared to aspirin alone (171). In addition, the CREDO trial showed that DAPT should be continued beyond the usual 30 days, as one year outcomes revealed a $27 \%$ risk reduction in death, $\mathrm{MI}$ and stroke in patients on DAPT compared to patients on aspirin alone past 30 days of DAPT (172). In the high-risk STEMI population, the use of DAPT before or after PCI in the CLARITY study resulted in significantly lower rates of cardiovascular death or ischaemic complications without an increase in major or minor bleeding (172). The doubling of clopidgorel dose to $600 \mathrm{mg}$ before $\mathrm{PCl}$ in the ARMYDA-2 study, significantly reduced periprocedural $\mathrm{MI}$ compared to patients receiving the conventional $300 \mathrm{mg}$. These studies in total show the superior efficacy of DAPT to prevent MACE in patients undergoing PCl compared to aspirin alone. Current guidelines advocate DAPT with aspirin and clopidogrel for up to 12 months depending on type of stent and favour continuation beyond this time period according to individual risk assessment (174).

There is also evidence to support clopidogrel monotherapy in PVD patients. In a subgroup analysis from CAPRIE, clopidogrel had its greatest effect on reducing cardiovascular events in patients with PVD (63). Clopidogrel has been approved for 
use in PVD, particularly in patients with adverse reactions or intolerance to aspirin (146).

\subsubsection{Clopidogrel HOTPR}

Despite remarkable improvements in patient outcomes following the implementation of DAPT, $8-10 \%$ of patients suffer a recurrent cardiovascular event during the first year following an ACS (175). It was first reported in 2002 by Jaremo et al. and then Gurbel et al. that recommended doses of clopidogrel produced very variable antiplatelet effects when given to different patients $(175,176)$. Then the following year, Matetzky et al. observed that this variability in response to clopidogrel correlated with clinical outcomes following PCI (178). In Gurbel et al's. early study in patients undergoing elective $\mathrm{PCl}$, platelet reactivity in response to ADP was measured at several time points, pre and post loading (clopidogrel $300 \mathrm{mg}$ ), 5 days and 30 days post $\mathrm{PCl}$ (176). HOTPR was classified as a $<10 \%$ fall from baseline and $31 \%$ of patients post loading and at day 5, fell in to this category. The prevalence of HOTPR dropped to $15 \%$ at day 30 post- $\mathrm{PCl}$, but there remained a wide inter-individual variability in the response to clopidogrel (176). The prevalence of clopidogrel HOTPR in coronary artery disease patients has since been the focus of a number of studies and rates vary between 25$40 \%$ depending on the assay used, the population tested and the timing of measurement post clopidogrel therapy $(178,179)$.

Several studies have linked clopidogrel HOTPR to increased risk of thrombotic events following $\mathrm{PCl}$ as summarised in Table 1-3. Early studies by Matetzky et al. using light transmission aggregometry (LTA) in STEMI patients undergoing $\mathrm{PCl}$, found patients in the lowest quintile of clopidogrel responsiveness had the highest rates of ischaemic events during follow up (178). Subsequently, it was suggested that high on treatment platelet reactivity to ADP would be a superior marker of ischaemic risk instead of the difference from baseline to on treatment, as platelet reactivity to ADP on aspirin alone was highly variable (181). The relationship between HOTPR and risk of ischaemic events was first prospectively investigated in the PREPARE POST-STENTING study (181). The presence of HOTPR was associated with a 2.6 increased risk of ischaemic 
events and the occurrence of MACE in patients without HOTPR was relatively rare, postulating a potential therapeutic target to protect patients against thrombotic complications post PCI. In 2008, Price et al. demonstrated patients with HOTPR (>235 PRU as measured by VerifyNow) had significantly higher rates of cardiovascular death $(2.8 \%$ vs. $0 \%, p=0.04)$ and stent thrombosis ( $4.6 \%$ vs. $0 \%, p=0.004)$ (182). Additionally, Sibbing et al. demonstrated a similar risk of ischaemic events associated with HOTPR as measured by the Multiplate analyser (184). Patients with HOTPR had a dramatic increased risk of stent thrombosis, OR 9.4 and cardiovascular death, OR 3.2 compared to normal responders at 30 days post $\mathrm{PCl}$. Many other studies have consistently demonstrated HOTPR to be an independent risk factor for ischaemic events post $\mathrm{PCI}$ (Table 1-3). The wealth of data on this subject also suggests that patients could potentially be protected from thrombotic/ischemic complications if low to moderate on treatment platelet reactivity were achieved.

There is limited data on the frequency of clopidogrel HOTPR in PVD patients. A small study looking at the incidence of HOTPR in 54 stable PVD patients found $32 \%$ had HOTPR on clopidogrel monotherapy and that this phenotype was not stable in all patients over time (184). The relationship between clopidogrel HOTPR and MACE had not been examined in a PVD population to our knowledge. 
Table 1-3 Studies linking HOTPR to adverse clinical events in CAD

\begin{tabular}{|c|c|c|c|c|}
\hline Study & $\begin{array}{l}\text { Patient group } \\
\text { (n) }\end{array}$ & Methods & $\begin{array}{l}\text { Definition of } \\
\text { HOTPR }\end{array}$ & Clinical Relevance \\
\hline $\begin{array}{l}\text { Barragan et } \\
\text { al. } \\
(185)\end{array}$ & $\mathrm{PCl}(46)$ & VASP-PRI & $>50 \%$ VASP & $\uparrow$ Stent thrombosis \\
\hline $\begin{array}{l}\text { Gurbel et al. } \\
(181)\end{array}$ & $\begin{array}{l}\text { Elective PCI } \\
\text { (192) }\end{array}$ & $\begin{array}{l}5 \mu \mathrm{M} \text { ADP - } \\
\text { LTA }\end{array}$ & $\begin{array}{l}\text { 75th percentile } \\
\text { post PCl } \\
\text { platelet } \\
\text { reactivity }\end{array}$ & $\begin{array}{l}\text { 个 post } \mathrm{PCl} \text { events } \\
\text { at } 6 \text { months, OR } 2.1\end{array}$ \\
\hline $\begin{array}{l}\text { Matetzky et } \\
\text { al.(186) }\end{array}$ & $\begin{array}{l}\text { STEMI-PCI } \\
(60)\end{array}$ & $\begin{array}{l}5 \mu \mathrm{M} \text { ADP - } \\
\text { LTA }\end{array}$ & $\begin{array}{l}\text { 75th percentile } \\
\text { platelet } \\
\text { reactivity }\end{array}$ & $\begin{array}{l}\text { 个 cardiac events at } \\
6 \text { months post } \mathrm{PCl}\end{array}$ \\
\hline $\begin{array}{l}\text { Bliden et al. } \\
\text { (187) }\end{array}$ & $\begin{array}{l}\text { Elective PCI } \\
(150)\end{array}$ & $\begin{array}{l}5 \mu \mathrm{M} \text { ADP - } \\
\text { LTA }\end{array}$ & $\begin{array}{l}\geq 50 \% \text { platelet } \\
\text { aggregation }\end{array}$ & $\begin{array}{l}\uparrow 1 \text { year post } \mathrm{PCl} \\
\text { events }\end{array}$ \\
\hline $\begin{array}{l}\text { Blindt et al. } \\
\text { (188) }\end{array}$ & $\begin{array}{l}\text { High risk for } \\
\text { ST - PCI (99) }\end{array}$ & $\begin{array}{l}\text { VASP-PRI } \\
\text { (72-96 hrs } \\
\text { after } \\
\text { stenting) }\end{array}$ & $>48 \%$ PRI & 个 6 month ST \\
\hline $\begin{array}{l}\text { Cuisset et al. } \\
\text { (190) }\end{array}$ & $\begin{array}{l}\text { NSTEMI -PCI } \\
(190)\end{array}$ & $\begin{array}{l}10 \mu \mathrm{M} \text { ADP- } \\
\text { LTA }\end{array}$ & $>70 \%$ LTA & $\begin{array}{l}\text { 个 periprocedural } \\
\text { ischaemia }\end{array}$ \\
\hline $\begin{array}{l}\text { Frere et al. } \\
(190)\end{array}$ & $\begin{array}{l}\text { NSTEMI - } \\
\text { PCI (195) }\end{array}$ & $\begin{array}{l}10 \mu \mathrm{M} \text { ADP- } \\
\text { LTA }\end{array}$ & $>70 \%$ LTA & $\begin{array}{l}\text { 个 } 30 \text { days post } \mathrm{PCl} \\
\text { events MACE + } \\
\text { Stroke }\end{array}$ \\
\hline $\begin{array}{l}\text { Geisler et al. } \\
\text { (191) }\end{array}$ & $\begin{array}{l}\text { CAD-PCl } \\
(802)\end{array}$ & $\begin{array}{l}20 \mu \mathrm{M} \text { ADP- } \\
\text { LTA }\end{array}$ & Upper quartile & $\begin{array}{l}\text { 个 } 30 \text { days MACE } \\
\text { and death } \mathrm{OR}=4.9\end{array}$ \\
\hline $\begin{array}{l}\text { Price et al. } \\
\text { (182) }\end{array}$ & $\mathrm{PCl}(380)$ & $\begin{array}{l}\text { VerifyNow } \\
\text { P2Y12 }\end{array}$ & $\geq 235$ PRU & $\begin{array}{l}\text { 个 } 6 \text { months post } \\
\text { PCl events } \\
\text { including ST }\end{array}$ \\
\hline $\begin{array}{l}\text { Gurbel et al. } \\
(192)\end{array}$ & $\begin{array}{l}\text { Elective PCl } \\
\text { (297) }\end{array}$ & $\begin{array}{l}5 \& 20 \mu \mathrm{M} \\
\text { ADP-LTA }\end{array}$ & $\begin{array}{l}>46 \% 5 \mu \mathrm{M} \\
\text { ADP } \\
>59 \% 20 \mu \mathrm{M} \\
\text { ADP }\end{array}$ & $\begin{array}{l}\uparrow 2 \text { year ischaemia } \\
\text { events } 5 \mu \mathrm{M} \text { OR }= \\
3.9,20 \mu \mathrm{M} \text { OR }= \\
3.8\end{array}$ \\
\hline $\begin{array}{l}\text { Bonello et al. } \\
(193)\end{array}$ & $\mathrm{PCl}(144)$ & VASP-PRI & $>50 \% \mathrm{PRI}$ & $\begin{array}{l}\text { 个 } 6 \text { months post } \\
\text { PCI MACE }\end{array}$ \\
\hline $\begin{array}{l}\text { Marcucci et } \\
\text { al. (194) }\end{array}$ & $\begin{array}{l}\text { PCl-ACS } \\
(683)\end{array}$ & $\begin{array}{l}\text { VerifyNow } \\
\text { P2Y12 }\end{array}$ & $\geq 240$ PRU & $\begin{array}{l}\text { 个 } 12 \text { month } \\
\text { ischaemic event, } \\
\mathrm{CV} \text { death OR = } \\
2.55 \text {, nonfatal } \mathrm{MI} \\
\mathrm{OR}=3.36\end{array}$ \\
\hline $\begin{array}{l}\text { Patti et al. } \\
\text { (195) }\end{array}$ & $\mathrm{PCl}(160)$ & $\begin{array}{l}\text { VerifyNow } \\
\text { P2Y12 }\end{array}$ & $\geq 240$ PRU & $\uparrow 1$ month MACE \\
\hline Sibbing et al. & $\begin{array}{l}\text { PCI-DES } \\
(1608)\end{array}$ & $\begin{array}{l}\text { Multiplate } \\
\text { Analyser (6.4 } \\
\mu \mathrm{M} \mathrm{ADP)}\end{array}$ & $\begin{array}{l}\text { Upper quintile } \\
\text { (>47 AU) }\end{array}$ & $\begin{array}{l}\text { 个 } 1 \text { month definite } \\
\text { ST, OR }=9.4\end{array}$ \\
\hline
\end{tabular}




\subsubsection{Clopidogrel LOWPR}

The prevalence and significance of low on treatment platelet reactivity (LOWPR) to ADP has not been as extensively studied as HOTPR. Early observations of higher bleeding rates in studies where more potent antiplatelet treatments were being administered suggested a negative trade-off of a poorer safety profile with improved clinical efficacy. In the CURE trial, DAPT was associated with a $38 \%$ increased relative risk of major bleeding compared to aspirin single therapy (196). Recent studies have investigated the relationship between LOWPR and bleeding risk following PCl. An enhanced response or hypersensitivity to clopidogrel as measured by the Multiplate in Sibbing et al. study, was associated with a 3.5 fold increased risk of procedure related major bleeding following $\mathrm{PCI}$ (197). In the Sibbing study, the investigators also found an association between HOTPR and ischaemic events, unveiling a spectrum of response to clopidogrel. In a study utilising the VerifyNow P2Y12 assay, the presence of LOWPR (PRU $\leq 86$ ) was associated with an increase in 1 month bleeding events in 507 patients tested undergoing PCI (198).

\subsubsection{Prasugrel}

Prasugrel is a second-generation thienopyridine pro-drug similar to clopidogrel. It is also metabolised by hepatic CYP isoenzymes into its active form, although prasugrel's chemical structure allows for a more efficient metabolism that is not as reliant on the CYP2C19 or CYP2C9 enzymes as clopidogrel (Figure 1-7) (199). This efficient metabolism results in a faster onset of action at doses' 10-100 fold lower than clopidogrel. A loading dose of $60 \mathrm{mg}$ reaches $80 \%$ inhibition of platelets 30 minutes after dosing, whereas clopidogrel even at a $600 \mathrm{mg}$-loading dose exerts minimal inhibition at the same time point (200). The anti-aggregatory effects of prasugrel lasts the lifespan of the platelet due to irreversible binding of the active metabolite to the P2Y12 receptor, similar to clopidogrel. Studies comparing prasugrel (60mg loading, $10 \mathrm{mg}$ maintenance) and clopidogrel (300mg loading, $75 \mathrm{mg}$ maintenance) in healthy subjects and patients with coronary artery disease consistently find a faster and higher 
mean platelet inhibition in participants receiving prasugrel (201-203). Although there exists a degree of variability in on-treatment platelet reactivity to prasugrel therapy, the prevalence of HOTPR on prasugrel is much lower than clopidogrel treatment (204). This is thought to be due to CYP2C19's limited role in the biotransformation process leading to a more consistent level of active metabolite between individuals rather than a greater potency of the active metabolite to inhibit the P2Y12 receptor (201) (202). In pharmacokinetic studies, low body weight and increasing age ( $\geq 75$ ) resulted in significantly greater platelet inhibition levels (205).

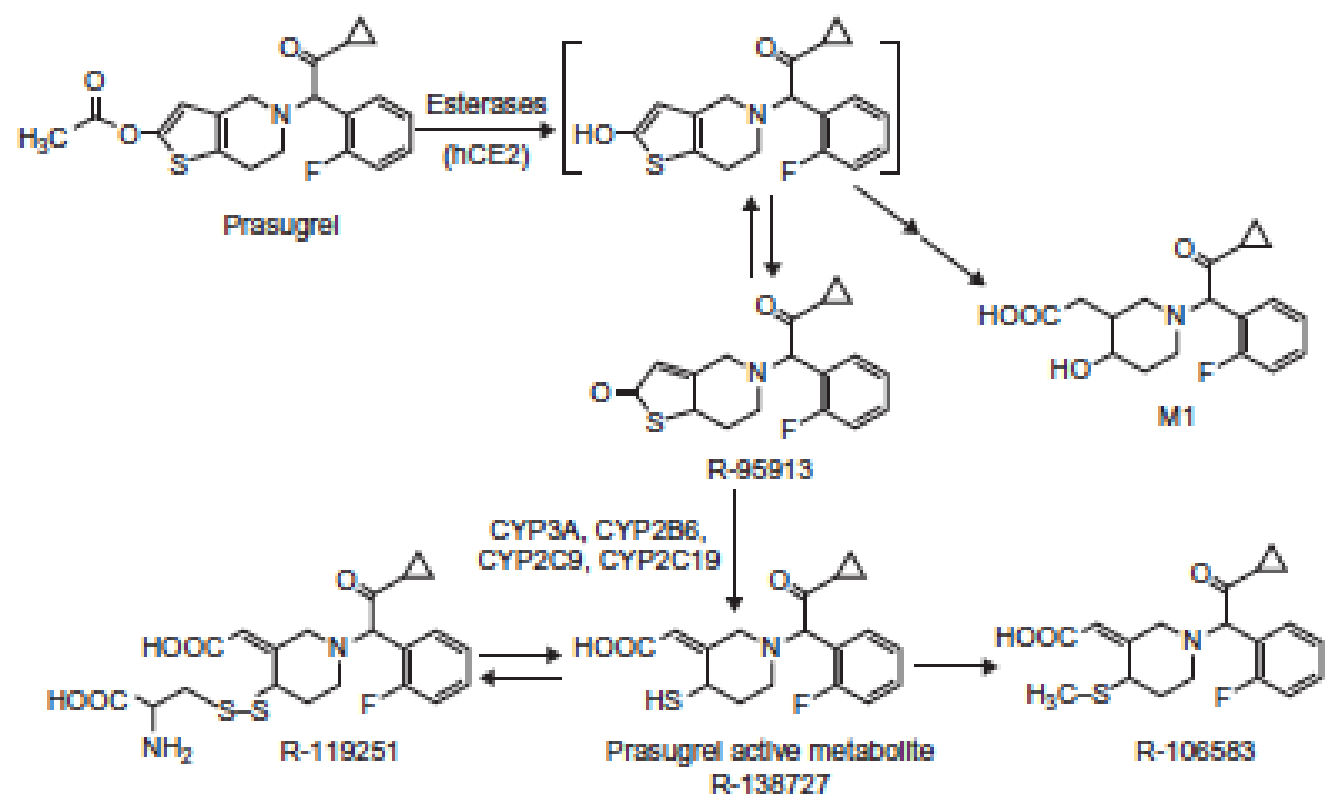

Figure 1-7 Metabolism of Prasugrel

Cattaneo M, ADP Receptor Antagonists, Platelet Third Edition, 2013; 1117-1138. Reproduced with permission from Elsevier.

The more potent efficacy of prasugrel compared to clopidogrel, was demonstrated in the TRITON-TIMI-38 trial, a phase III trial that evaluated 13,608 high-risk ACS patients undergoing PCl (206). Patients were randomised to receive either prasugrel (60mg loading, 10mg maintenance) or clopidogrel (300mg loading, $75 \mathrm{mg}$ maintenance) for 6 - 15 months. Prasugrel was associated with a 19\% risk reduction in ischaemic events (HR 0.81, p < 0.001) but a higher incidence of major and fatal bleeding complications (HR 1.32, $p=0.03)$. Landmark analysis revealed a significant protection from ischaemic events particularly in the perioprocedural time period, but decreased ischaemic rates 
also extended out to the end of the study (207). There was a notable increase in the incidence of major bleeding in patients undergoing CABG surgery on prasugrel compared to clopidogrel and post hoc analysis indicated when prasugrel was withheld 7 days before surgery, the rates of major bleeding were substantially reduced (207). Other post-hoc analyses also identified subgroups that either received more benefit or more harm from prasugrel therapy. Patients with previous cerebrovascular accidents, patients over 75 years of age or weighing less than $60 \mathrm{~kg}$ appeared to have less of a net clinical benefit and/or greater harm than the same subgroups receiving clopidogrel. Consequently, adjustment of the maintenance dose to $5 \mathrm{mg}$ is recommended in these populations and validity of this approach is the subject of ongoing studies (208). Patients with diabetes, STEMI, coronary stents, or recurrent cardiovascular events on treatment all had significantly more favourable outcomes compared to the same subgroups on clopidogrel $(209)(210,211)$. Based on the results of the TRITON TIMI-38 trial, prasugrel was FDA approved and is recommended in highrisk patients for a short time period (213).

\subsubsection{Ticagrelor}

Ticagrelor is a direct and reversible P2Y12 antagonist and belongs to the new chemical class cyclopentyl-triazolo-pyrimidines. Interestingly, ticagrelor binds to the P2Y12 receptor via a mechanism that is non-competitive to ADP, suggesting the existence of an independent alternative-binding site on the receptor (213). Ticagrelor also has a rapid onset of action, achieving maximal platelet inhibition in about 2 hours and the achieved platelet inhibition levels are less variable compared to clopidogrel $(214,215)$. Unlike clopidogrel and prasugrel, ticagrelor does not undergo hepatic metabolism and is ingested in its active form resulting in the observed fast onset and consistent platelet inhibition.

The DISPERSE-2 study compared the safety of ticagrelor with that of clopidogrel in 990 NSTEMI patients on top of aspirin therapy for up to 12 weeks (217). There was no 


\section{INTRODUCTION}

difference in major bleeding observed between the two groups. The study did confirm that dyspnea occurred more frequently with ticagrelor and in this study the impact on discontinuation of treatment was low. The mechanism of this side effect remains unclear and some have hypothesised that it is mediated by the induced increase in adenosine release. Whether this side effect will impact more in a real world setting of ticagrelor administration is unknown. Ticagrelor was shown to decrease the incidence of cardiovascular death, $\mathrm{Ml}$ or stroke by $16 \%$ compared to clopidogrel ( $9.8 \%$ vs. $11.7 \%$ ) in NSTEMI and STEMI patients in the PLATO study (217). This was a high-risk population and two thirds were undergoing $\mathrm{PCl}$. The more potent ticagrelor also reduced the secondary endpoints of $\mathrm{MI}(5.8 \%$ vs. $6.9 \%)$ and unexpectedly, cardiovascular death $(4.0 \%$ vs. $5.1 \%)$. Whilst in the overall study there was no difference in bleeding between the two treatment groups, the incidence of major nonCABG related bleeding was significantly higher in the ticagrelor treated patients (219). In patients undergoing CABG the incidence of both cardiovascular and total mortality was lower in the ticagrelor treated patients, while the incidence of major CABGbleeding was similar, potentially due to the quicker regain of platelet function after discontinuation of ticagrelor (219). Therefore, similar to the TRITON-TIMI 38 trial, the PLATO trial demonstrated that more potent and consistent P2Y12 inhibition of platelet function than clopidogrel is associated with greater antithrombotic protection but a higher risk of non-CABG major bleeding. Based on the results of the PLATO trial, ticagrelor is now FDA-approved.

\subsubsection{Novel P2Y12 inhibitors}

Several other novel antiplatelet agents are in development for the use in ACS, some of these are outlined in Table 1-4. Cangrelor and elinogrel are both new P2Y12 receptor antagonists. Cangrelor has undergone two phase III trials, CHAMPION-PCI (220) and CHAMPION-PLATFORM (221) that have evaluated the intravenous and oral form of cangrelor. Both trials were terminated early, as there was insufficient evidence to show cangrelors efficacy. Another phase III trial, CHAMPION-PHOENIX is 
currently under way to evaluate cangrelors efficacy with outcomes. Elinogrel has shown promising results in the phase II INNOVATE-PCI trial with greater and more rapid antiplatelet action compared to clopidogrel and no excess bleeding, as is common with stronger platelet inhibition (222). The current targets of antiplatelet agents have either been COX-1 or $\mathrm{P}_{2} \mathrm{Y}_{12}$ platelet activation pathways. Patients continue to have events on these antiplatelet agents and this may be due to the known upregulation of other pathways that are left uninhibited.

\subsubsection{Other platelet inhibitors under clinical trial}

Several drugs including cilostazol, protease-activated-receptor-1 (PAR-1) antagonists and oral anticoagulants have been suggested as adjunct therapies to aspirin and a $\mathrm{P} 2 \mathrm{Y}_{12}$ inhibitor. Cilostazol is a phosphodiesterase III inhibitior, that effects platelet intracellular cAMP levels. It has shown some promise in diabetic patients (OPTIMUS2) with a significant decrease in measured $P 2 Y_{12}$ activation levels compared to control. However, a multitude of side effects has inhibited its development (223). PAR-1 antagonists, atopaxar and vorapaxar are at varying stages of development (225) and could be particularly relevant in ACS populations, as thrombin generation processes are enhanced in these patients (225). Early development of revacept, a soluble dimeric Glycoprotein VI-Fc fusion protein, that interferes with collagen induced platelet activation has been shown to inhibit platelet aggregation in a dose dependent manner without altering general homeostasis (226). 
INTRODUCTION

Table 1-4 Major novel antiplatelet agents in development

\begin{tabular}{|c|c|c|c|c|c|}
\hline Drug & Class & Platelet inhibition target & Administration & Half life & Development stage \\
\hline Cangrelor & $\begin{array}{l}\text { ADP P2Y } 12 \\
\text { receptor } \\
\text { antagonist }\end{array}$ & $\begin{array}{l}\text { Direct, reversible } \mathrm{P}^{2} \mathrm{Y}_{12} \\
\text { inhibition }\end{array}$ & Intravenous & $3-5 \operatorname{mins}$ & $\begin{array}{l}\text { Phase III trial -CHAMPION- } \\
\text { PHOENIX }\end{array}$ \\
\hline Elinogrel & $\begin{array}{l}\text { ADP P2Y } 12 \\
\text { receptor } \\
\text { antagonist }\end{array}$ & $\begin{array}{l}\text { Competitive, direct and } \\
\text { reversible } \mathrm{P}_{2} \mathrm{Y}_{12} \text { inhibition }\end{array}$ & Intravenous and oral & $12 \mathrm{hrs}$ & Phase III trial \\
\hline Vorapaxar & $\begin{array}{l}\text { Thrombin PAR-1 } \\
\text { antagonist }\end{array}$ & Direct, reversible & Oral & $165-311 \mathrm{hrs}$ & $\begin{array}{l}\text { Phase III trial - TRACER and } \\
\text { TRA-2P }\end{array}$ \\
\hline Atopaxar & $\begin{array}{l}\text { Thrombin PAR-1 } \\
\text { antagonist }\end{array}$ & Direct, reversible & Oral & $22-26$ hrs & $\begin{array}{l}\text { Phase II (LANCELOT-ACS } \\
\text { and LANCELOT-CAD) } \\
\text { complete }\end{array}$ \\
\hline Revacept & $\begin{array}{l}\text { GP V1 pathway } \\
\text { blocker }\end{array}$ & $\begin{array}{l}\text { Interferes with collagen } \\
\text { mediated platelet activation }\end{array}$ & Intravenous & $67-137 \mathrm{hrs}$ & Completed phase I trial \\
\hline
\end{tabular}

Key: PAR-1, Protease-activated receptor-1; GP, Glycoprotein. 


\subsubsection{HOTPR and LOWPR risk factors}

The variability in response to clopidogrel is multi-factorial and complex, influenced by genetic factors, clinical variables and non-compliance. A schematic diagram of these factors is depicted in Figure 1-8.

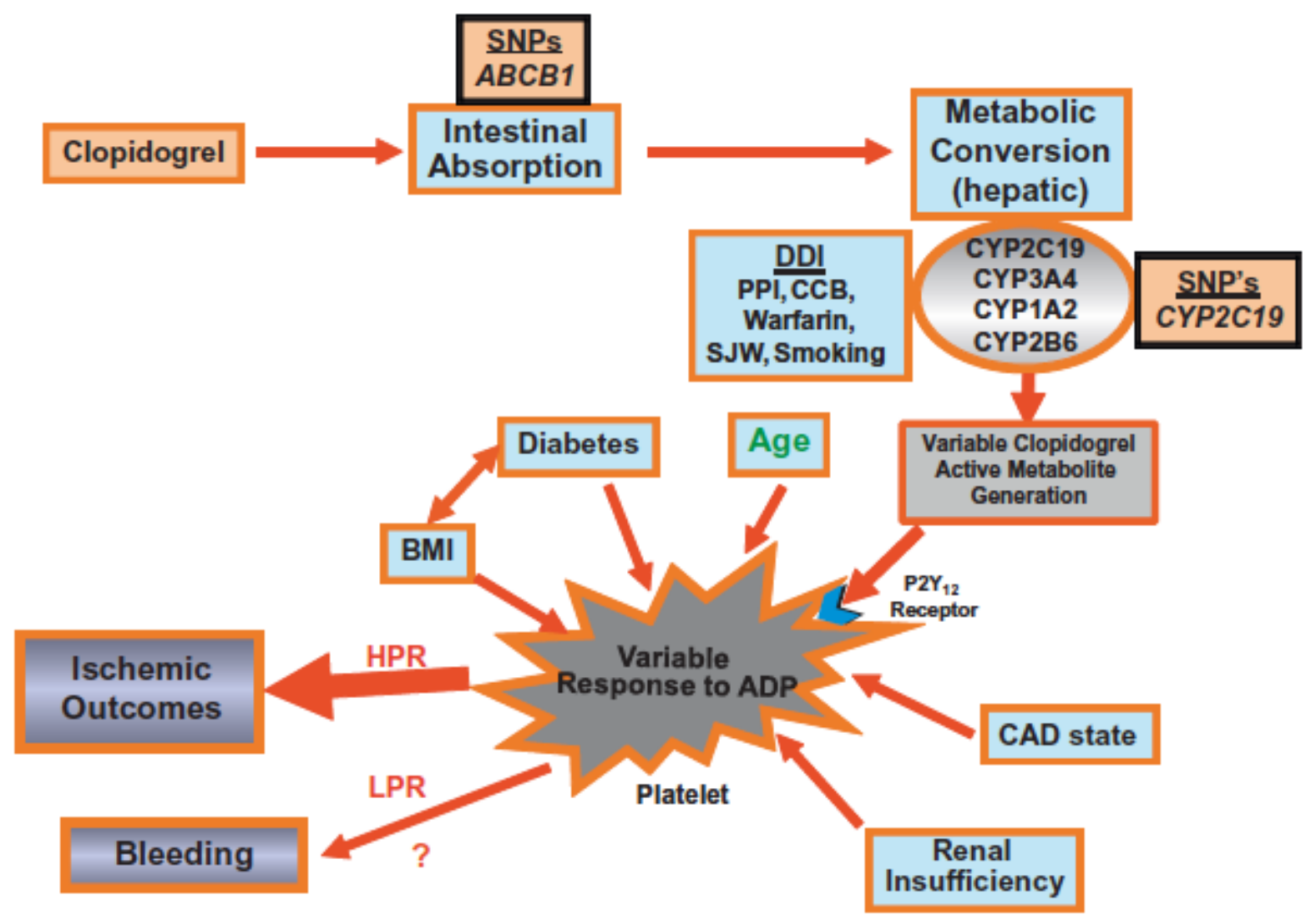

Figure 1-8 Factors influencing clopidogrel metabolism and ADP response.

The pharmacodynamic response to clopidogrel is influenced by numerous factors such as single nucleotide polymorphisms of genes encoding p-glycoprotein transporter (ABCB1) and CYP2C19 and also by drugs that are metabolized by hepatic cytochrome enzymes along with clopidogrel (e.g., proton pump inhibitors, calcium channel blockers, St. John's Wort, smoking). In addition, platelet response to ADP is also influenced by age, body mass index, diabetes, coronary artery disease state, and renal insufficiency. The variable response to ADP results in increased ischemic outcomes in the presence of high platelet reactivity and bleeding in the presence of low platelet reactivity. BMI, body mass index; CAD, coronary artery disease; CCB, calcium channel blockers; CYP, cytochrome; DDI, drug-drug interaction; HPR, high platelet reactivity; LPR, low platelet reactivity; PPI, proton pump inhibitor; SNP, single nucleotide polymorphism; SJW, St. John's Wort. Gurbel PA and Tantry US, Monitoring of antiplatelet therapy, Platelet Third Edition, 2013; 603-633. Reproduced with permission from Elsevier 


\subsubsection{Genetic Factors}

Several genetic mutations or single nucleotide polymorphisms (SNPs) have been identified in genes that control clopidogrel absorption and metabolism and could affect the level of active metabolite that is produced. Several SNPs, at least 25, have been identified in the gene encoding the CYP2C19 isoenzyme, which influence the catalytic activity of the enzyme is a dose-dependent manner. Of the SNPs identified in the CYP2C19 gene, the most widely analysed and most frequent are $* 2$, a $G \rightarrow A$ mutation in exon 5 producing an aberrant splice site, leading to the complete absence of CYP2C19 activity; and $* 17(-806 \mathrm{C} \rightarrow \mathrm{T})$, a regulatory region variant that has been associated with increased expression and enzymatic activity. The * 2 is the most common loss-of-function (LOF) allele whereas *3, and *4, *5, *6, *7, and *8 are less common LOF alleles (227).

The prevalence of the * 2 LOF allele differs between ethnic groups, occurring in $13 \%$ of Caucasians, $18 \%$ of African Americans and $29 \%$ of East Asians (228). Importantly, in a NZ population a single small study has identified a higher frequency of the *2 LOF Maori (24\%) compared to NZ Europeans (15\%) (230). On the other hand, the *17 GOF allele has found to be more prevalent in Caucasians (21\%) compared to African Americans (16\%) and Asians (4\%) (228). Carriage of at least one CYP2C19 LOF allele was demonstrated to decrease both active metabolite levels of clopidogrel in the blood by $34 \%$ (relative reduction, $p<0.001$ ) and platelet inhibition ( $9 \%$ absolute reduction from baseline, $\mathrm{p}<0.001$ ) compared to non-carriers in healthy participants (231). Homozygous LOF allele carriage has the greatest impact on ADP induced platelet reactivity resulting in a significant increase in on treatment platelet reactivity. Platelet function between heterozygous diplotypes is highly variable and therefore genotyping will fail to identify a large proportion of patients who have HOTPR (231).

The impact of CYP2C19*2 carriage on MACE risk is debatable in the literature with some metanalyses reporting an increase in cardiovascular risk, particularly stent thrombosis in patients undergoing percutaneous coronary intervention (PCI) (232, 233). However, other studies have directly challenged this link and report a marginal 
increase in MACE for patients with the LOF allele $(234,235)$. The reported inconsistencies linking CYP2C19 and MACE are most likely due to the small influence $(5-12 \%)$ that the LOF allele has on the variability in clopidogrel response (236).

The gain of function allele CYP2C19*17 is associated with significantly lower levels of ADP-induced platelet aggregation and a higher risk for bleeding during clopidogrel treatment compared to wild-type carriers (237). However, in this study conducted by Sibbing et al, platelet function was not reported in those who had bleeding and whether the GOF allele affects cardiovascular events remains unclear.

Genetic polymorphisms also affect other genes that are central to active clopidogrel metabolite production. Limited intestinal absorption and therefore decreased hepatic biotransformation of clopidogrel has been associated with the homozygous $3435 \mathrm{C}$ to T mutation of ATP binding cassette sub family B member 1 ( $A B C B 1)$. The gene encodes the efflux pump P-glycoprotein and is central to clopidogrel absorption in the gut (238). It has more recently been proposed that paraoxonase-1 (PON-1) is the crucial enzyme involved in clopidogrel bioactivation and polymorphisms in this gene lead to higher rates of HOTPR (239), but this finding was not confirmed by several subsequent studies (240).

\subsubsection{Drug-Drug Interactions}

Several drug interactions with the P450 isoenzymes have shown to contribute to the variability in clopidogrel metabolite production. The coadministration of clopidogrel with proton pump inhibitors, calcium channel blockers, St. John's wort, smoking and warfarin, which are metabolised by the CYP2C19, CYP3A4, CYP1A2 and CYP2C9 isoenzymes may potentially influence clopidogrel response (242).

Patients treated with clopidogrel for cardiovascular disease, are frequently also on proton pump inhibitors (PPIs) and both drugs are mainly metabolised by CYP3A4 and CYP2C19 isoenzymes. Consequently, recent studies have focused on the relation of PPI treatment to clopidogrel metabolism and cardiovascular outcomes. Platelet 
function studies have found an association between omeprazole administration and reduced clopidogrel response, as measured by the VASP phosphorylation, but no change with pantoprazole or esomeprazole administration $(242,243)$. Several retrospective studies have observed higher rates of major adverse cardiovascular events in patients receiving both clopidogrel and a PPI suggesting a decrease in clopidogrel's ability to prevent ischaemic events (245). However, this finding has not been consistent and other studies have found no association leaving a lack of conclusive evidence to support a clinically important interaction between clopidogrel and PPI use.

\subsubsection{Diabetes}

Diabetes Mellitus (DM) is a metabolic disorder that is accompanied by a prothrombotic state and cardiovascular disease is the leading cause of mortality in this patient group (246). Diabetic patients not only have a higher cardiac risk than nondiabetics but also suffer from more cardiovascular complications and recurrent atherothrombosis events $(245,246)$. Multiple mechanisms have been suggested to contribute to the platelet dysfunction observed in DM patients and are caused by metabolic and cellular abnormalities (247-250). This dysfunction results in two important factors that negatively impact diabetic patients: a higher level of baseline platelet reactivity and higher levels of antiplatelet drug targets compared to nondiabetics

These include the enhanced production of thromboxane due to increased arachidonic acid metabolism and observed low intra cellular levels of glutathione and other antioxidants in diabetic platelets $(251,252)$. Calcium homeostasis is also disrupted leading to increased cytosolic calcium concentration and platelet hyperaggreability (254). Due to the hyperglycemic environment in diabetic patients, platelet function is augmented in many ways. There is enhanced expression of P-selectin (254) and glycation of certain platelet surface proteins such as GPIIb/IIla, which may decrease membrane fluidity and increase platelet adhesion $(255,256)$. 
Nitric oxide (NO) production by platelets inhibits interactions with the endothelium and promotes endothelium mediated vasodilation in normal conditions (258). NO synthase levels are significantly decreased in diabetic patients (259) although interestingly, levels can be improved by the presence of insulin (259). Furthermore, this deficiency of insulin and/or its response on tissues that is central to diabetes, contributes to platelet dysfunction from the abolishment of insulin's plateletinhibitory effects $(260,261)$. Recent research has shown a striking upregulation of the

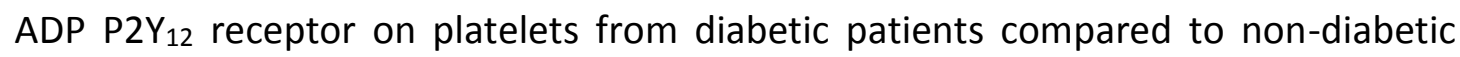
controls, leading to increased adhesion, aggregation and procoagulant activity (262, 263). Lastly, diabetic patients have a higher turnover of platelets, with the presence of larger, sensitive reticulated platelets, resulting in platelet hyperactivity and lower response to antiplatelet agents (264-266). 
Table 1-5 Mechanisms contributing to platelet dysfunction in diabetes

\begin{tabular}{|l|}
\hline Increased production of thromboxane $\mathrm{A}_{2}$ \\
\hline $\begin{array}{l}\text { Increased expression of platelet surface adhesion molecules, including; CD31, } \\
\text { CD4ab, CD62P and CD63. Leads to an increase in platelet activation. }\end{array}$ \\
\hline Increase in platelet-dependent thrombin generation \\
\hline $\begin{array}{l}\text { Increased expression of platelet surface receptors P-selectin, GPIb and GPIIlb/lla. } \\
\text { GPIllb/IIla binds fibrinogen } \\
\text { Important step in thrombogenesis }\end{array}$ \\
\hline $\begin{array}{l}\text { Decrease vascular synthesis of NO and PGI } \\
\text { aggregation opposed to vasoconstriction. }\end{array}$ \\
\hline $\begin{array}{l}\text { Disorded calcium homeostasis - effect shape change, secretion, aggregation and } \\
\text { thromboxane formation. }\end{array}$ \\
\hline $\begin{array}{l}\text { Decrease platelet insulin receptor number and affinity. } \\
\text { Insulin is thought to decrease platelet response to ADP, collagen, thrombin, } \\
\text { arachidonate and PAF }\end{array}$
\end{tabular}

Key: GP = glycoprotein; $v W f=$ von Willebrand factor; PGI2 = prostacyclin; NO = nitric oxide; $\mathrm{ADP}=$ adenosine diphosphate; $\mathrm{PAF}=$ platelet-activating factor

\subsubsection{Obesity}

Increased BMI has also been associated with an increase in HOTPR rates. Furthermore, there appears to be a linear relationship, with increasing BMI leading to higher levels of platelet reactivity in obese patients (267). A contributing factor to the high rates of HOTPR in obese patients is the underdosing of clopidogrel in relation to body weight. However, studies have found even triple doses of $600 \mathrm{mg}$ of clopidogrel in some obese patients has not been sufficient to overcome HOTPR suggesting additional factors contributing to the observed high platelet reactivity (268). These include an increased inflammatory state and enhanced PAR-1 medicated platelet activation and impaired hepatic metabolism of clopidogrel due to fatty liver disease (268).

\subsubsection{Renal Insufficiency}

Renal insufficiency is associated with increased rates of HOTPR on clopidogrel therapy (269). A confounding factor is the high rate of diabetes in renal failure patients, which is the key risk factor for nearly half of end stage renal failure patients and as previously 
described is a known contributor to HOTPR (270). However, a recent study observed a significant association between renal insufficiency, as measured by estimated glomerular filtration rate (eGFR) and residual platelet reacitivty with the highest rates of HOTPR in patients with a eGFR of $<15 \mathrm{ml} / \mathrm{min} / 1.73 \mathrm{~m}^{2}$. This finding was independent of diabetes as a significant predictor of HOTPR but only in patients with an eGFR below 15 (271). The mechanism to which renal insufficiency fosters HOTPR is still unknown.

Other variables that influence the response to clopidogrel include: advanced age, CAD state and non-compliance (238). More recently the role of microRNAs which are involved in regulating gene expression, was investigated in platelet reactivity in a study by Rui Shi et al (273). The microRNA miR-223 expression in this study was found to negatively correlate with platelet reactivity in patients on clopidogrel. The expression of miR-233 in platelets, correlated with higher levels of P2Y12 expression and consequently with high levels of HOTPR and was a significant predictor of HOTPR in $33 \mathrm{PCl}$ patients. Future studies with larger populations are needed to test the impact of this miRNA on clopidogrel response. 


\subsection{Platelet Function Testing}

The diverse response to antiplatelet drugs and its link with clinical outcomes has driven the development and implementation of platelet function testing in an effort to improve clinical outcomes. There are several tests available (outlined in Table 1-6 and Figure 1-9) that differ significantly in predictive value of clinical outcome (238), consumable and testing costs and their appropriateness as a point of care assay for real time platelet function monitoring (274).

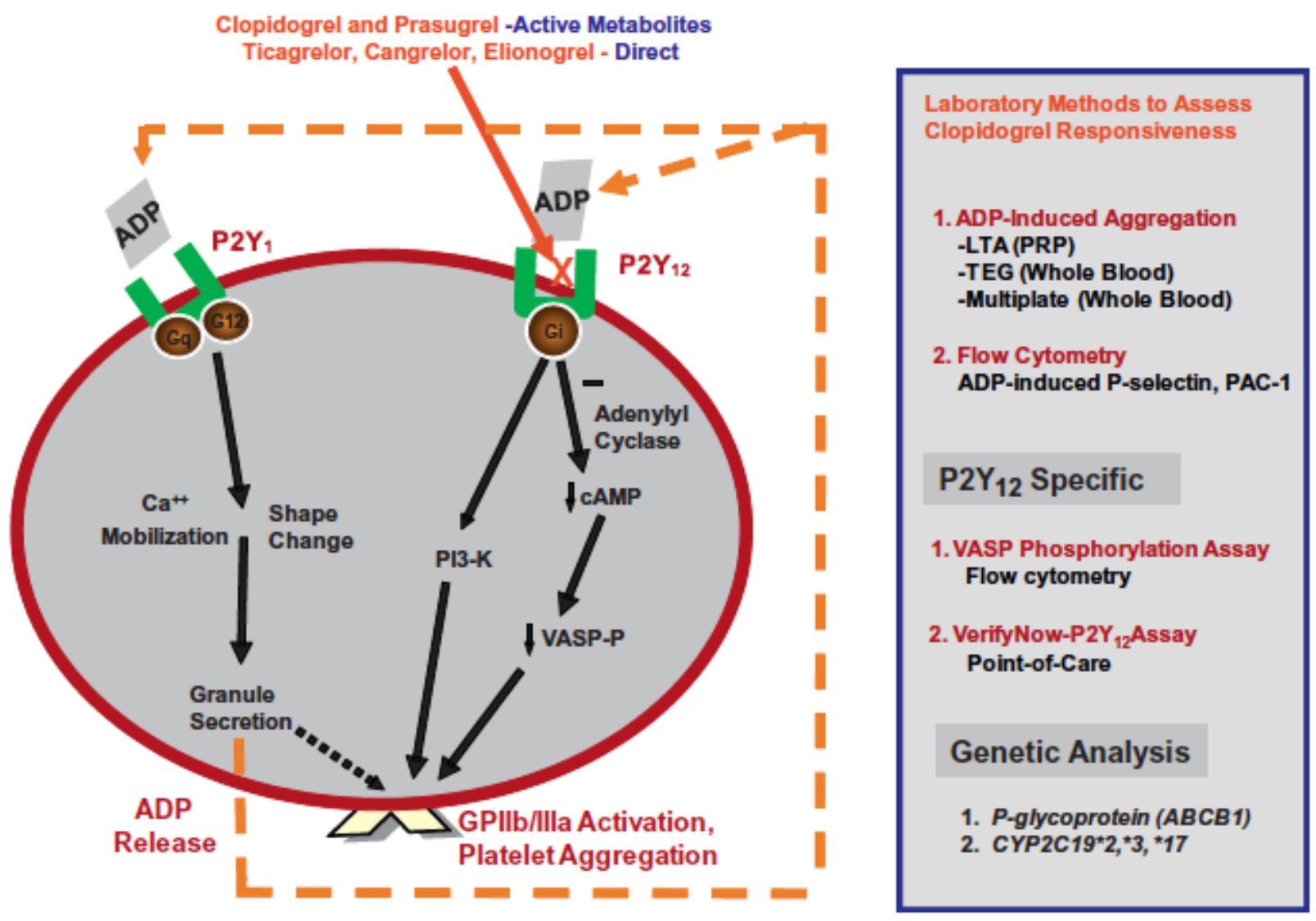

Figure 1-9 Laboratory measurement of on treatment platelet reactivity.

Laboratory methods to assess clopidogrel responsiveness are conducted by either measuring the response to ADP or specifically measuring the amount of P2Y12 inhibition. Genetic analysis of polymorphisms in the genes associated with metabolism of clopidogrel can also be performed. The ADP and P2Y12 specific methods can also be used to measure the response to other antiplatelet agents such as: prasugrel, ticagrelor, cangrelor and elinogrel which are all P2Y12 receptor antagonists. ADP, adenosine diphosphate; cAMP, cyclic adenosine monophosphate; CYP, cytochrome; PRP, platelet-rich plasma; LTA, light transmittance aggregometry; PI3K, phosphatidylinositol 3-kinase; TEG, throm- belastography; VASP, vasodilator-stimulated phosphoprotein; VASP-P, phosphorylated vasodilator-stimulated phosphoprotein. Gurbel PA and Tantry US, Monitoring of antiplatelet therapy, Platelet Third Edition, 2013; 603-633. Reproduced with permission from Elsevier. 
Table 1-6 Platelet Function Assays

\begin{tabular}{|c|c|c|c|c|c|}
\hline Assay & Methodology & $\begin{array}{l}\text { Platelet } \\
\text { stimulation }\end{array}$ & Advantages & Disadvantages & $\begin{array}{l}\text { Diagnostic cutoff } \\
\text { point for ADP (ROC) }\end{array}$ \\
\hline LTA & $\begin{array}{l}\text { Reduction of optical density } \\
\text { after stimulation in PRP }\end{array}$ & $\begin{array}{l}\text { ADP, collagen, } \\
\text { AA, TRAP }\end{array}$ & $\begin{array}{l}\text { Good predictivity, } \\
\text { historical measure }\end{array}$ & $\begin{array}{l}\text { Complex sample prep, } \\
\text { time consuming }\end{array}$ & $\mathrm{n} / \mathrm{a}$ \\
\hline VASP & $\begin{array}{l}\text { Flow cytometric detection } \\
\text { of VASP phosphorylation }\end{array}$ & $\begin{array}{l}\text { ADP and } \\
\text { ADP + PGE1 } \\
\text { in parallel }\end{array}$ & $\begin{array}{l}\text { Whole blood assay, } \\
\text { P2Y } 12 \text { receptor specific }\end{array}$ & $\begin{array}{l}\text { Technically complex method } \\
\text { need flow cytometer, } \\
\text { weak sensitivity and } \\
\text { predictivity }\end{array}$ & $\mathrm{PRI}>50 \%$ \\
\hline VerifyNow & $\begin{array}{l}\text { Platelet mediated aggregation } \\
\text { of fibrinogen-coated } \\
\text { polystyrene beads }\end{array}$ & $\begin{array}{l}\text { ADP + PGE1 } \\
\text { (P2Y12 assay) } \\
\text { AA (Aspirin assay) } \\
\text { (Ilb/Ila assay) }\end{array}$ & $\begin{array}{l}\text { Whole blood assay, } \\
\text { true point of care, } \\
\text { standardized procedure }\end{array}$ & $\begin{array}{l}\text { No room for assay } \\
\text { adjustment, expensive } \\
\text { cartridges }\end{array}$ & $\mathrm{PRU}>235-240$ \\
\hline $\begin{array}{l}\text { Multiplate } \\
\text { Analyser }\end{array}$ & $\begin{array}{l}\text { Impedance aggregometry } \\
\text { coating of } 2 \text { electrode pairs } \\
\text { by platelets }\end{array}$ & $\begin{array}{l}\text { ADP, ADP + PGE1, } \\
\text { AA, TRAP-6 }\end{array}$ & $\begin{array}{l}\text { Whole blood assay, } \\
\text { simple and rapid, } \\
\text { standardized procedure, } \\
\text { good predictivity for } \\
\text { bleeding and stent } \\
\text { thrombosis }\end{array}$ & $\begin{array}{l}\text { Requires pipetting, } \\
\text { samples time sensitive }\end{array}$ & $>47 \mathrm{AU}$ \\
\hline
\end{tabular}

Abbreviations: ADP, adenosine diphosphate; PGE1, prostaglandin E1. AU indicates aggregation units; LTA, light transmittance aggregometry; ROC, receiver operator characteristic curve analysis; PRI, platelet reactivity index; PRU, P2Y12 reaction units; VASP, vasodilator-stimulated phosphoprotein phosphorylation analysis 


\subsubsection{Platelet function assays}

Laboratory based methods require specialised facilities, skilled personnel and can be time consuming. Examples of these include, Light transmission aggregometry (LTA) (274), which measures the optical density after preparation of platelet rich plasma and stimulated with an agonist. Historically, it has been viewed as the 'gold standard' of platelet function testing, and although it has good predictivity of clinical outcomes, the assay remains complex with no standardisation between testing (276). Another laboratory-based method, VASP phosphorylation measured by flow cytometry, is a whole blood assay that specifically measures $\mathrm{P} 2 \mathrm{Y}_{12}$ receptor antagonism. Similarly to LTA, this assay is complex with the requirement of specialised equipment and time consuming. Unlike, LTA the VASP assay has weak sensitivity and predictivity (276).

On the other hand, point-of-care assays are rapid and less complex, which are more desirable features when integrating these assays into clinical practice. The two most commonly used point-of-care assays are the VerifyNow Assay and the Multiplate analyser. The VerifyNow assay is a turbidimetric assay that measures aggregation of platelets to fibrinogen-coated beads in whole blood (182). The Multiplate analyser is an impedance aggregometer that assesses platelet function in whole blood (277). Both these assays are rapid and simple, with results obtained in less than 10 minutes and can be used to test residual platelet reactivity to a range of agonists. Testing procedures are completely standardised allowing comparable results between operators and institutions. The Multiplate is semi-automated, requiring pipetting and has been found to strongly predict bleeding and stent thrombosis risk (277), with a recent study displaying it was more predictive of ischaemic events than the VASP assays (278). On the other hand, VerifyNow is completely automated but consequently requires expensive testing cartridges. It is becoming more apparent that while laboratory-based assays are valuable for platelet function research purposes, only the rapid, standardised and easy to use methods will be appropriate for wide spread clinical use.

Diagnostic cut-point values for high on treatment platelet reactivity have been determined for the reviewed platelet function assays using receiver operating 
characteristic curve analysis (Table 1-3) and these are recommended by the Working Group on High On-Treatment Platelet Reactivity (238). Irrespective of the assay used, these cutoffs are a strong predictor of recurrent ischaemic events such as myocardial infarction or stent thrombosis. Furthermore, according to a recent meta-analysis of 20 studies utilising the described platelet function tests, a patient identified with HOTPR has a 3.4 fold higher risk of cardiovascular death compared to a patient with an adequate ADP response (279). As with recurrent ischaemic risk, bleeding risk has also been linked with low platelet reactivity or hypersensitivity in patients on clopidogrel (192). The TRITON TIMI 38 trial (280) and recent Sibbing et al. (282) study suggest there is a therapeutic window for antiplatelet therapy in general and specifically P2Y12 receptor inhibition with "normal platelet reactivity" showing the lowest risk of either bleeding or stent thrombosis as measured by the Multiplate (Figure 1-10).

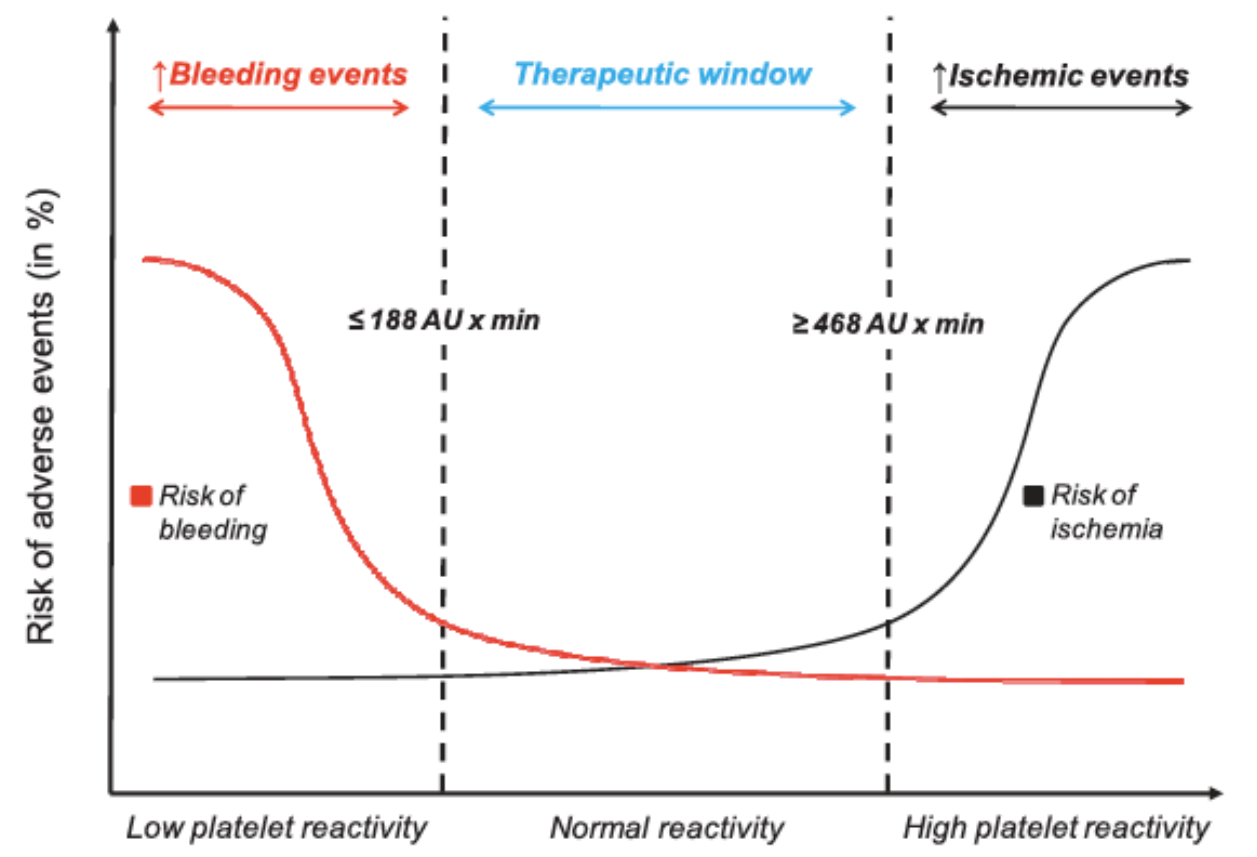

Levels of P2Y12 receptor reactivity

Figure 1-10 Therapeutic window of P2Y12 blockade

Sibbing et al. study suggest there is a therapeutic window for antiplatelet therapy in general and specifically P2Y12 receptor inhibition with "normal platelet reactivity" showing the lowest risk of either bleeding or stent thrombosis as measured by the Multiplate Sibbing et al. Platelet Aggregation and Its Association With Stent Thrombosis and Bleeding in Clopidogrel-Treated Patients. Initial Evidence of a Therapeutic Window. Journal of the American College of Cardiology. (2010) Vol 56: 4:317-318. Reproduced with permission 


\section{INTRODUCTION}

\subsubsection{Personalised antiplatelet therapy}

Following the observed link between HOTPR and increased adverse outcomes, several studies have aimed to improve patient outcome by modifying antiplatelet therapy and lowering platelet reactivity. Treatment of HOTPR on maintenance clopidogrel therapy has been attempted by using higher loading or maintenance doses of clopidogrel, adding cilostazol, switching to more potent alternative $P 2 Y_{12}$ receptor blockers such as prasugrel or ticagrelor, or by adding elinogrel or GPIIb/IIla antagonists (283). Two small studies by Bonelle et al. randomised patients undergoing $\mathrm{PCl}$ to a guided antiplatelet therapy using the VASP assay or unguided therapy. Those who were found to have HOTPR in the guided arm were given an additional $600 \mathrm{mg}$ loading of clopidogrel. This treatment was successful in reducing on treatment platelet reactivity and decreased the rate of early stent thrombosis without an increase in major bleeding compared to a non-guided approach $(283,284)$. The selective addition of GPIIb/IIla antagonists on top of DAPT in PCI patients with HOTPR has also been shown to improve patient outcomes without increasing bleeding (285). These early studies suggested that a platelet reactivity threshold of increased thrombotic and ischaemic risk could be used to target therapy and improve clinical outcomes.

However, this outcome was not observed in the three largest randomised trials using platelet function testing to tailor antiplatelet therapy. The GRAVITAS trial was the first large scale trial $(n=2214)$ that utilised the VerifyNow P2Y12 assay to identify patients with HOTPR and subsequently doubled clopidogrel dosing(287). High-dose clopidogrel treatment was ineffective in reducing the 6-month composite ischemic event occurrence (cardiovascular death, nonfatal MI, and stent thrombosis); both treatment groups had an unexpectedly low event rate (2.3\%). Similarly, the ARCTIC study enrolled 2440 patients undergoing $\mathrm{PCI}$ to receive guided therapy with the VerifyNow or non-guided therapy. Several different treatments were available to clinicians in the guided arm including reloading with clopidogrel (600 mg), switching to prasugrel or administration of a GPIIb/IIla inhibitor during $\mathrm{PCl}$. The study was also neutral with no improvement in clinical outcomes for patients receiving guided therapy compared to standard clopidogrel therapy without monitoring (287). The TRIGGER-PCI trial, which 
compared prasugrel versus clopiodgrel in patients with HOTPR as identified by the VerifyNow, was stopped by the steering committee prematurely after randomisation of 423 patients (out of the planned 2150) due to an interim analysis indicating a lower than expected incidence in the primary endpoint. Given the low rate of ischemic events, the clinical utility of this strategy could not be demonstrated (288).

Many have taken the results of these trials and concluded the platelet reactivity is not a modifiable risk factor that can be treated to improve patient outcome. However, there are several issues with the study design of these trials that may have contributed to the negative results. These include, recruiting a study population with a low risk of thrombotic complications, the assay used to determine tailored therapy may not have been sensitive enough and the use of antiplatelet treatment that provided inadequate therapy to overcome HOTPR. Therefore, it remains a fundamental issue to characterise patient populations using the right platelet function assay and cut-point, to identify a population that may benefit from the use of proper protocols of personalised antiplatelet therapy. 


\subsection{Overall aims and objectives}

This thesis explores the presence and prevalence of high and low residual platelet reactivity in patients with atherosclerotic disease and the different clinical, demographic and genetic factors that influence this phenotype. Furthermore, which factors are predictive of a patient's outcome and could be used to inform a patient's risk or tailor antiplatelet therapy. In addition to assessing the extent to which platelet function testing is useful as a risk-marker in patients, the wider interactions between platelets and lymphocytes contributing to the inflammatory state of a patient with atherosclerosis could also influence risk. Therefore, interactions between platelets and $T$ cells were examined.

The overall aims of the thesis are:

1. To understand the reproducibility of the Multiplate assay and the different methodological factors that add variance when measuring residual platelet reactivity

2. To examine the incidence of HOTPR in a NZ ACS population and the impact of prasugrel to reduce high on treatment residual platelet reactivity.

3. To investigate the independent clinical predictors of high residual platelet reactivity as well as the prevalence and impact of genetic variants in the CYP2C19 gene on platelet reactivity.

4. To examine the relationship between phenotype, genotype and predictors of phenotype with MACE and bleeding events in an ACS population.

5. To investigate the relationship between residual platelet reactivity on aspirin as well as the cardiac biomarkers hs-TnT, NT-proBNP and CRP with cardiovascular and bleeding outcomes in patients undergoing major vascular surgery.

6. To discern differences in platelet-T cell interactions in patients with atherosclerotic vascular disease compared to healthy controls. 
7. To study the impact of platelet interactions on CD4 T cell differentiation and the effect of antiplatelet therapy on platelet $-\mathrm{T}$ cell interactions 
INTRODUCTION 
2 Chapter 2 - Measurement of platelet reactivity 


\subsection{Introduction}

Several assays are available to measure platelet reactivity that differ in their complexity, price and time to deliver results as reviewed in section 1.5.1. We have chosen to use the Multiplate in a series of clinical studies to investigate platelet reactivity within this thesis. To use this assay clinically we need to understand the reproducibility of the measurement and the impact of methodological factors on the precision of the assay. There is substantial variation in the Multiplate methodology of reported studies to date, and the effect of these methodological variations on the reported results is unknown.

Thus, the aims of the study were:

1. To assess how reproducible the assay is in ADP and Arachadonic Acid (AA) stimulated platelets.

2. To determine the effect of time delay from sample collection to testing on platelet reactivity.

3. How the choice of anticoagulant effects the measurement of platelet reactivity.

4. If the use of a manual pipette can improve the analytical precision of the assay. 


\subsection{Methods}

\subsubsection{Study Population}

Patients presenting to our institution were eligible for inclusion in the study if they presented with an ACS, there was an invasive approach (coronary angiography +/- $\mathrm{PCI}$ ) planned and they were adequately pretreated with aspirin and clopidogrel. ACS was defined as symptoms suggestive of ischaemia lasting $>10 \mathrm{~min}$ in duration coupled with either a troponin elevation or new $1 \mathrm{~mm}$ ST segment deviation or T wave inversion in at least 2 contiguous leads (289). Adequate pretreatment was defined as chronic therapy with aspirin $(\geq 75 \mathrm{mg})$ and clopidogrel $(\geq 75 \mathrm{mg}$ ) or loading with clopidogrel $\geq$ $300 \mathrm{mg}$ at least 6 hours before or aspirin $\geq 300 \mathrm{mg}$ at least 2 hours before enrolment.

Exclusion criteria included a platelet count less than $100 \times 10^{9} / \mathrm{L}$, known platelet function disorder, administration of a fibrinolytic agent within 24 hours of enrolment and administration of a glycoprotein IIb/IIla receptor antagonist within a week prior to enrolment. The study was reviewed and approved by the Upper South A Regional Ethics Committee and all patients provided written informed consent (URA/11/05/016).

\subsubsection{Blood sampling and platelet function testing}

Blood for platelet function testing was collected from a peripheral vein using a 21gauge needle before angiography or collected in the cardiac catheterization laboratory from the arterial sheath immediately after insertion and before administration of heparin. All samples were collected in tubes containing huridin (25 $\mu \mathrm{g} / \mathrm{ml}$, Dynabyte, Munich, Germany) and were tested $30 \mathrm{~min}$ post collection unless otherwise stated.

Residual platelet aggregation in whole blood was assessed with multiple electrode impedance aggregometry using the Multiplate analyser (Figure 2-1), following the manufacturer's instructions. 


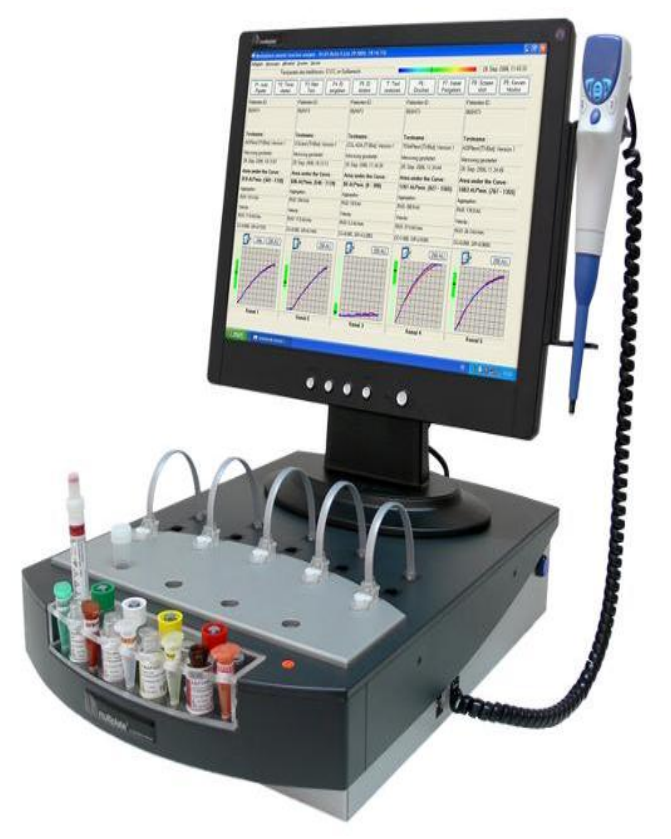

Figure 2-1 Multiplate Analyser System

Images recreated from Multiplate educational material http://www.multiplate.net/en/detection.php.

Whole blood was added directly to the test cuvette (Figure 2.2A) and diluted 1:1 with $0.9 \% \mathrm{NaCl}$ solution to give a final volume of $600 \mu \mathrm{L}$. Inside each test cell is a Teflon coated magnetic stirring bar (Figure 2-2A) and samples were stirred for 3 minutes at $37^{\circ} \mathrm{C}$. After stirring $20 \mu \mathrm{L}$ of $0.2 \mathrm{mM}$ ADP (Dynabyte, Munich, Germany) was added to the test cell with a final concentration of $6.4 \mathrm{mM}$. The Multiplate automatic pipette, which delivers 20-300 $\mu \mathrm{L}$ volumes, was used for reagent delivery unless stated otherwise. Addition of an agonist stimulates platelets to aggregate to the paired electrodes in the test cells and impede the current across them (Figure 2-2B \& C). 


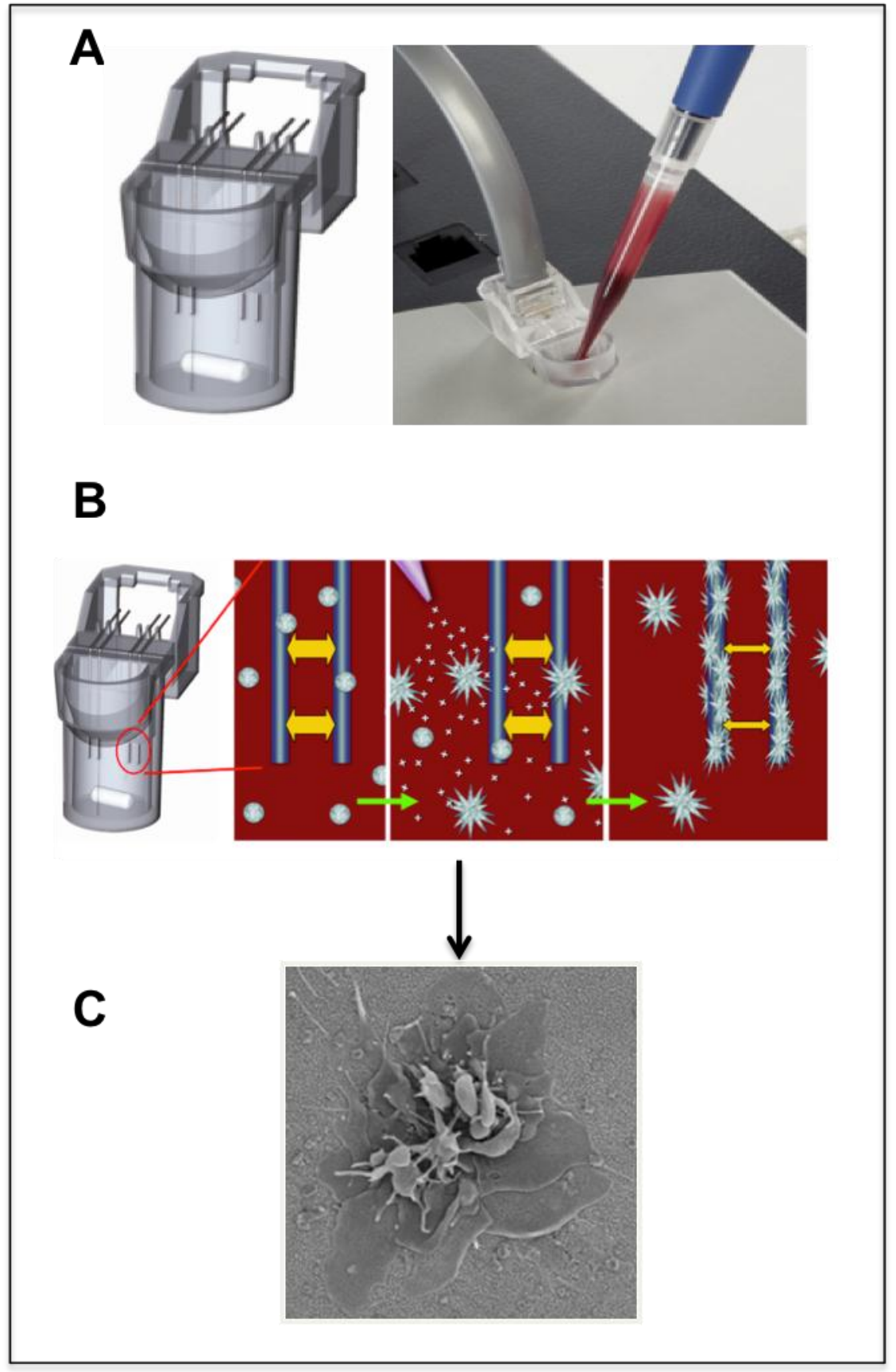

Figure 2-2 Multiplate electrode impedance aggregometry

Whole blood and saline are added to test cuvette using the automated pipette (A). Paired electrodes are present in each test cell and with the addition of platelet agonists, platelets are activated and aggregates form on electrodes and impede the current (B). Electron Scanning Microscopy image of platelet aggregates on electrode surface (C). Images recreated from Multiplate educational material http://www.multiplate.net/en/detection.php.

The increase of impedance due to the attachment of platelets to the electrodes is detected for each sensor unit separately and was continuously recorded for 6 minutes (Figure 2-3). The change in impedance is measured over time and the area under the curve (AUC) is transformed to arbitrary units and platelet reactivity is expressed in AU. 
Both pairs of electrodes (Test 1 and 2 ) are measured and the difference in these tests acts as an internal quality check.

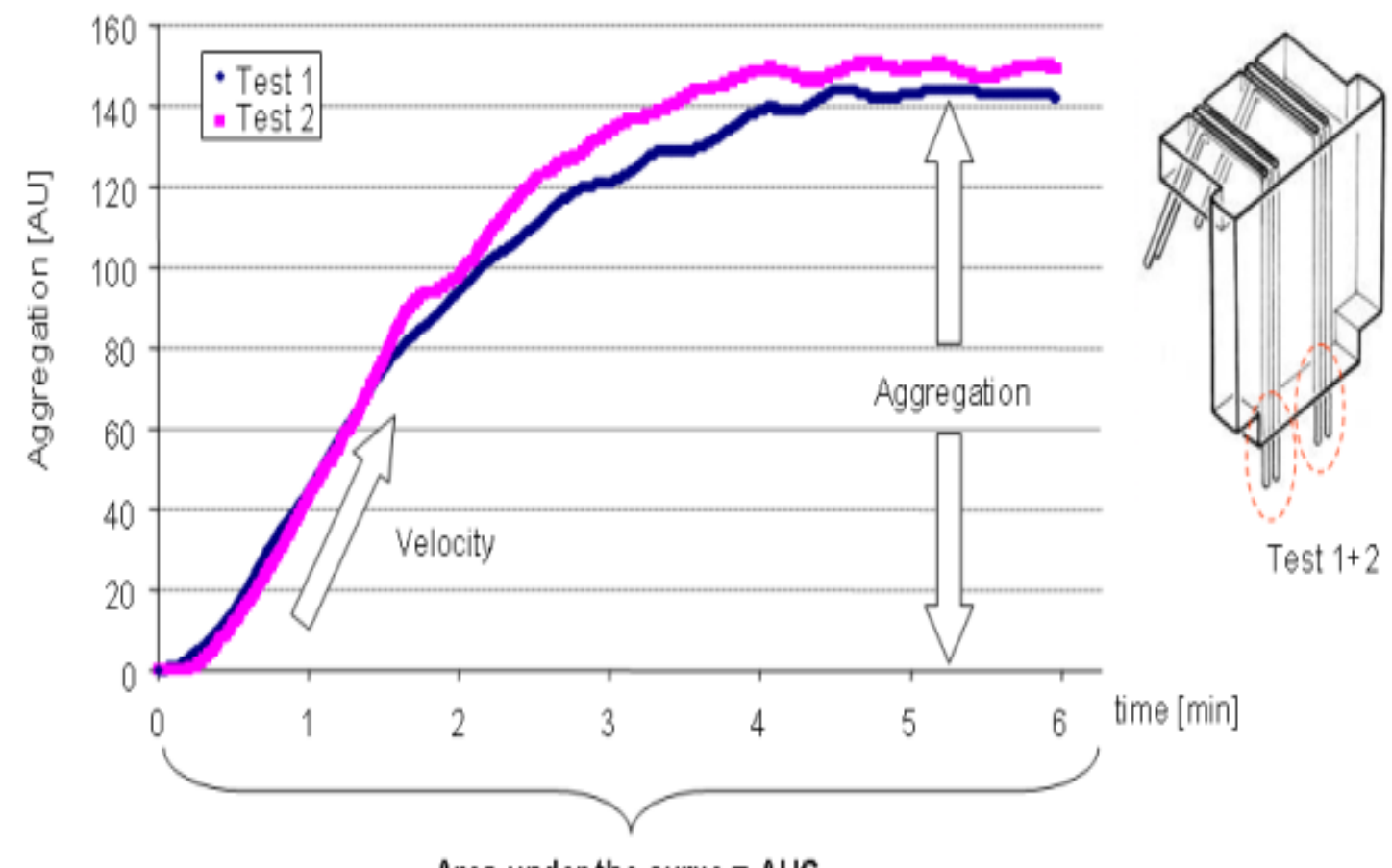

Area under the curve $=$ AUC

Figure 2-3 Aggregation over time in Multiplate test

Impedance is measured from each electrode pair (Test 1 and Test 2 ) and aggregation is plotted over time. The area under the curve is then converted in to arbitrary units and platelet reactivity is expressed as AU.

\subsubsection{Experimental Design}

Using the above platelet function testing protocol and the following changes, we tested reproducibility of measurement, effect of different anticoagulants, time sensitivity and the use of a manual pipette on variance in the following 4 studies.

\subsubsection{Protocol 1 - Analytical Precision}

Blood was drawn from 30 patients and tested using the above Multiplate protocol. In addition to ADP stimulation, the reproducibility of Arachidonic acid (AA) stimulation was also tested by the addition of $20 \mu \mathrm{L}$ of $15 \mathrm{mM}$ stock AA (Dynabyte, Munich, Germany) in addition to the standard Multiplate protocol with a final concentration of $0.5 \mathrm{mM}$. The coefficient of variation (CV) between each pair of measurements was then calculated. 
2.2.3.2 Protocol 2 - Choice of anticoagulated blood tube

To determine the effect of different anticoagulants on platelet aggregation, blood from 20 patients was collected into tubes containing 3 different anticoagulants; hirudin $(25 \mu \mathrm{g} / \mathrm{ml}$, Dynabyte, Munich, Germany), lithium heparin $(15 \mathrm{U} / \mathrm{ml} \mathrm{BD}$ Vacutainer, NJ, USA) and sodium citrate (0.109M, BD Vacutainer, NJ, USA). Blood from each tube was tested in a 1:1 dilution with $0.9 \% \mathrm{NaCl}$. In addition, blood anticoagulated with sodium citrate was tested in a 1:1 dilution with $3 \mathrm{mmolL} \mathrm{CaCl}_{2} /$ $0.9 \% \mathrm{NaCl}$ solution.

\subsubsection{Protocol 3 - Time delay to testing}

To test the effect of time delay between sample collection and Multiplate testing on platelet aggregation, blood was drawn from 20 patients and tested using the standard Multiplate protocol at the following 6 time intervals post blood sampling: 15, 30, 45, 60, 120 and 180 minutes.

\subsubsection{Protocol 4 - Use of Manual Pipette}

To test the effect of using a manual pipette on the analytical precision of the assay, blood was collected from 20 patients into a hirudin tube and tested at 30 minutes in two parallel tubes using calibrated manual pipettes. A $1 \mathrm{~mL}$ pipette was used for $\mathrm{NaCl}$ and whole blood, and a $100 \mu \mathrm{L}$ pipette for ADP.

\subsubsection{Statistics}

Continuous variables are reported as mean ( \pm standard deviation). Statistical analysis was performed using student's t-test, one-way ANOVA or linear regression where appropriate. Statistical significance was taken at $5 \%$. Calculations were performed using the GraphPad Prism Software package (GraphPad Software Inc, CA, USA). 


\subsection{Results}

\subsubsection{Analytical Precision}

The CV for 30 samples anticoagulated with hirudin and analysed using the standard Multiplate protocol plus ADP stimulation is shown in Figure 2-4. The mean CV for ADP induced platelet aggregation was $10.8 \%$ ( $95 \% \mathrm{Cl}, 7.5 \%$ - 14.1\%). Arachadonic Acid (AA) stimulated platelet aggregation was also tested (Figure 2-5) and the mean CV was $11 \%$ $(95 \% \mathrm{Cl}, 8.3 \%-15.3 \%)$. There was no relationship between coefficient of variation and level of platelet aggregation with either ADP or AA stimulation.

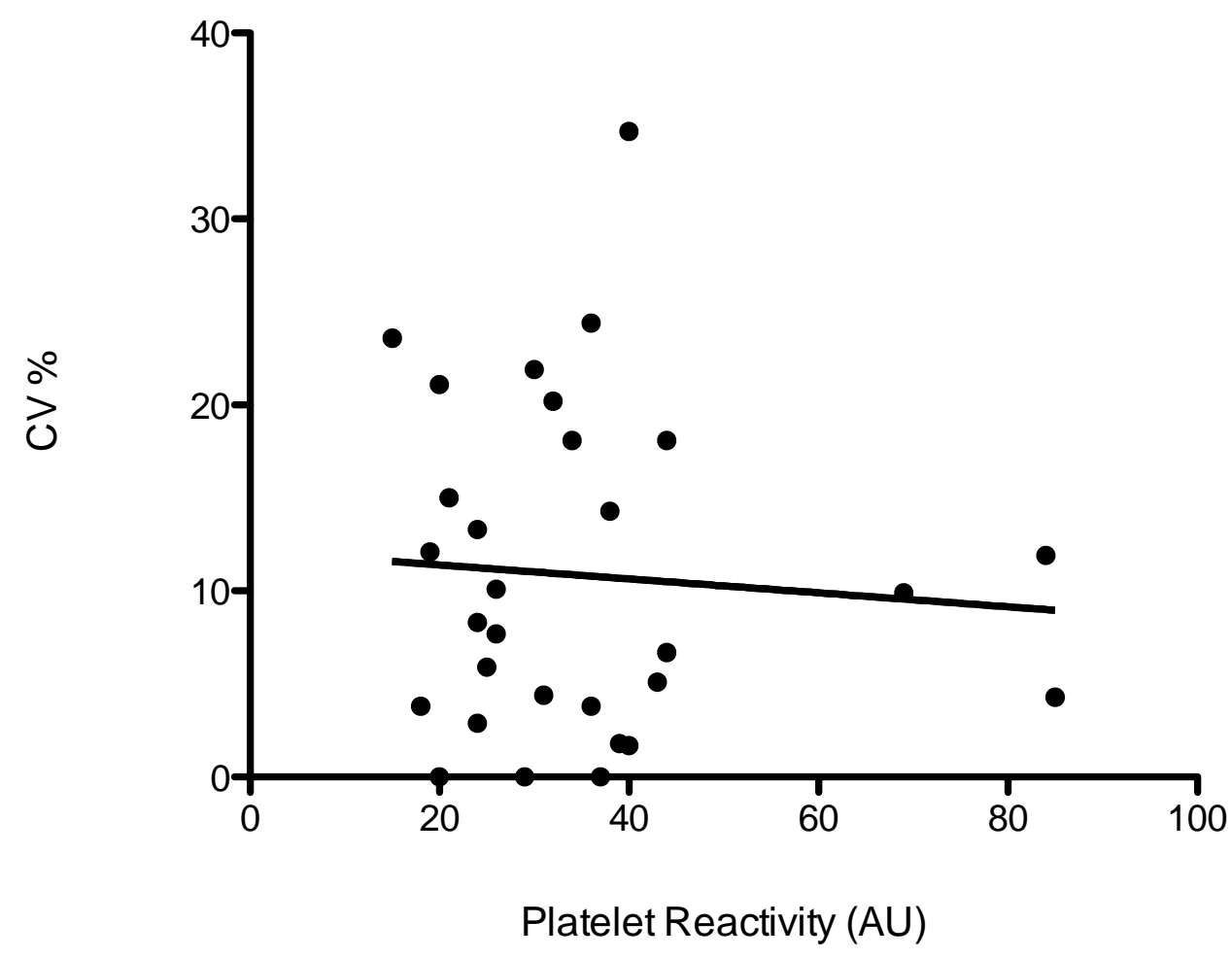

\section{Figure 2-4 Coefficient of variance (CV) in platelet aggregation using standard} Multiplate protocol with ADP stimulation.

The CV was calculated by the standard deviation divided by the mean of the two tests from the same patient sample was expressed as CV\% and plotted against absolute platelet aggregation $(\mathrm{AU})$. There was no correlation between increased variance with the increase in platelet aggregation $\left(r^{2} 0.005\right)$. Each point represents duplicate patient samples $(n=30)$. 


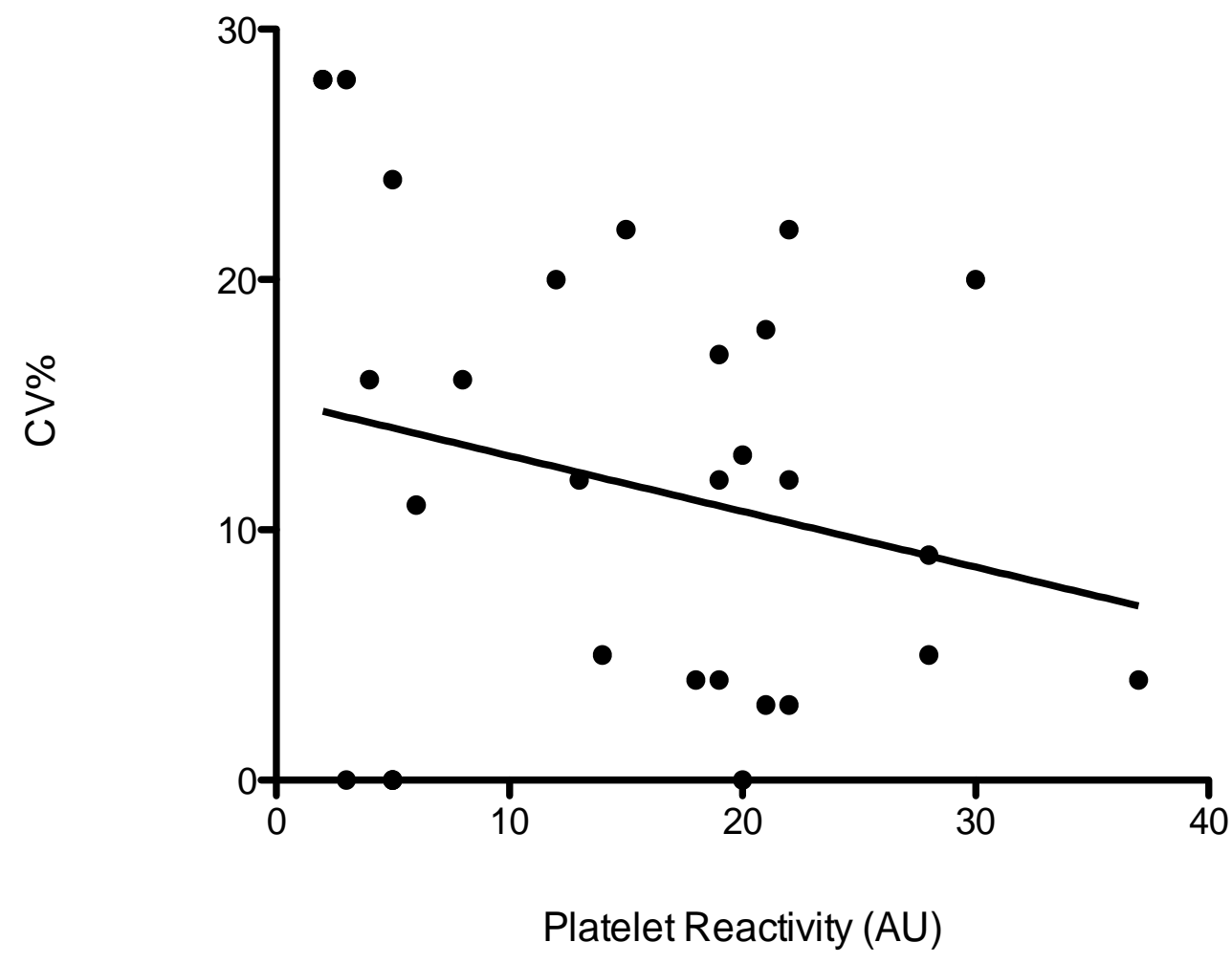

Figure 2-5 Coefficient of variance (CV) in platelet aggregation using standard Multiplate protocol with Arachadonic Acid (AA) stimulation.

The CV was calculated by the standard deviation divided by the mean of the two tests from the same patient sample, expressed as $\mathrm{CV} \%$ and plotted against absolute platelet aggregation (AU). There was no correlation between increased variance with the increase in platelet aggregation $\left(r^{2} 0.05\right)$. Each point represents duplicate patient samples $(n=30)$. 


\subsubsection{Choice of anticoagulant}

The choice of anticoagulant had a marked effect on residual platelet aggregation

(Figure 2-6). Platelet aggregation was increased in heparin-treated whole blood (52.0 $\pm 32 \mathrm{AU})$ when compared to hirudin $(36.5 \pm 31 \mathrm{AU}, \mathrm{P}=0.0015)$, with an average relative increase of $65 \%$. Conversely, platelet aggregation was reduced in blood treated with sodium citrate $(25.0 \pm 21 \mathrm{AU})$, resulting in an average reduction of $30.6 \%$ when compared to hirudin $(P=0.003)$. However, addition of $\mathrm{CaCl}_{2}$ to the citratetreated blood restored platelet aggregation $(34.0 \pm 25 \mathrm{AU}, \mathrm{P}=0.45)$ to levels similar to those seen with hirudin anticoagulation.

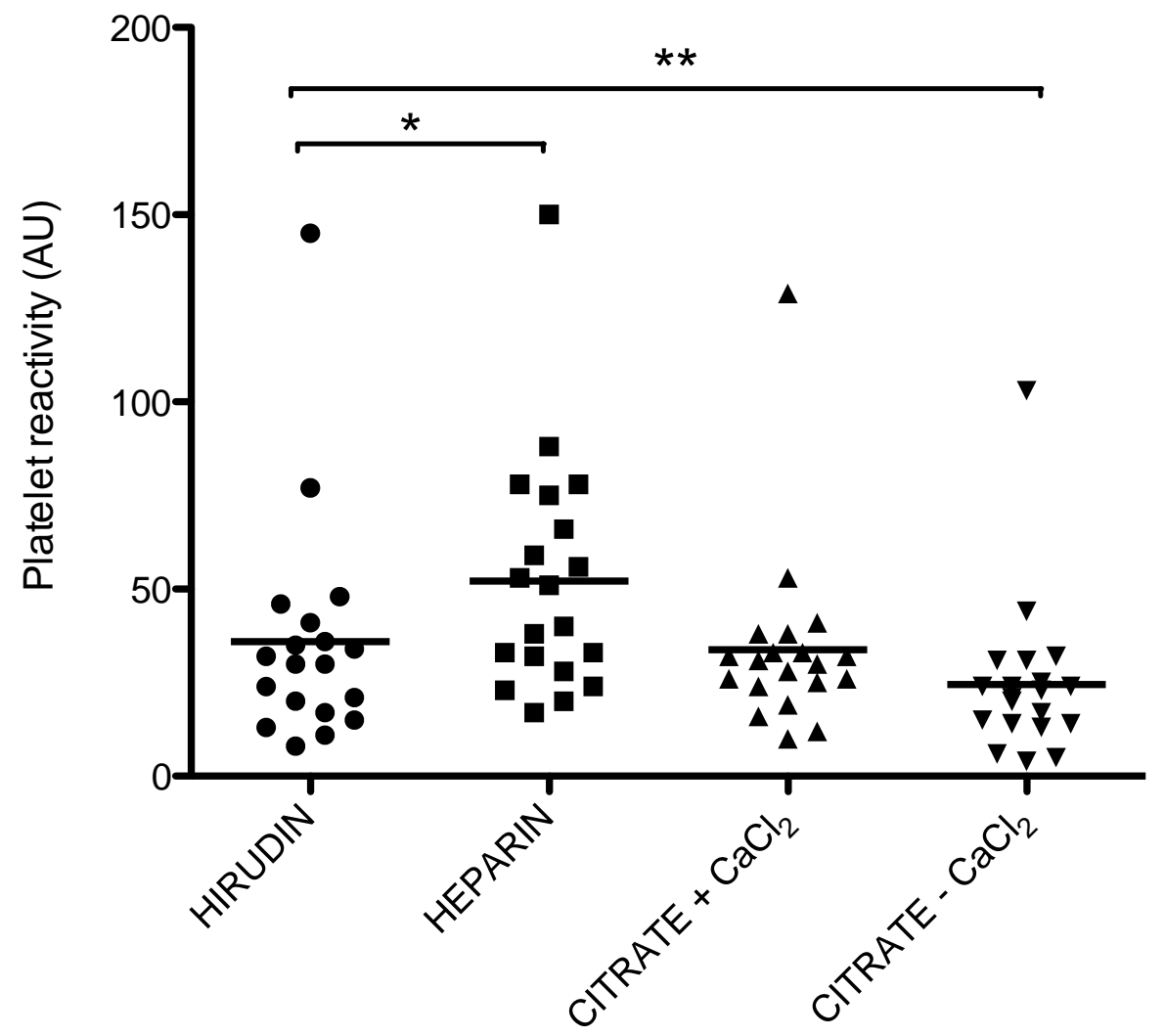

Anticoagulant

Figure 2-6 Effect of anticoagulant on variance in platelet aggregation.

Platelet aggregation tested using whole blood collected into tubes containing hirudin, heparin and citrate. Citrate-treated blood was tested with and without $\mathrm{CaCl}_{2} .{ }^{*} \mathrm{P}=0.0015$, ${ }^{* *} \mathrm{P}=0.0034$. 


\subsubsection{Effect of time delay between sampling and testing}

Platelet aggregation was sensitive to the time delay between sampling and analysis

(Figure 2-7). There was no difference in platelet aggregation at 15 minutes (38.8 29 $\mathrm{AU}, \mathrm{P}=0.614)$ or 45 minutes $(37.1 \pm 27 \mathrm{AU}, \mathrm{P}=0.377)$ compared to 30 minutes $(62.0$ $\pm 35 \mathrm{AU})$. However, when platelet aggregation at 30 minutes was compared to $60 \mathrm{~min}$ (34.2 $\pm 25 \mathrm{AU}, \mathrm{P}<0.05), 120$ minutes $(19.8 \pm 12 \mathrm{AU}, \mathrm{P}<0.001)$ and 180 minutes $(16.7$ $\pm 9 \mathrm{AU}, \mathrm{P}<0.001)$, there was a significant reduction in aggregation, especially the last two-time points.

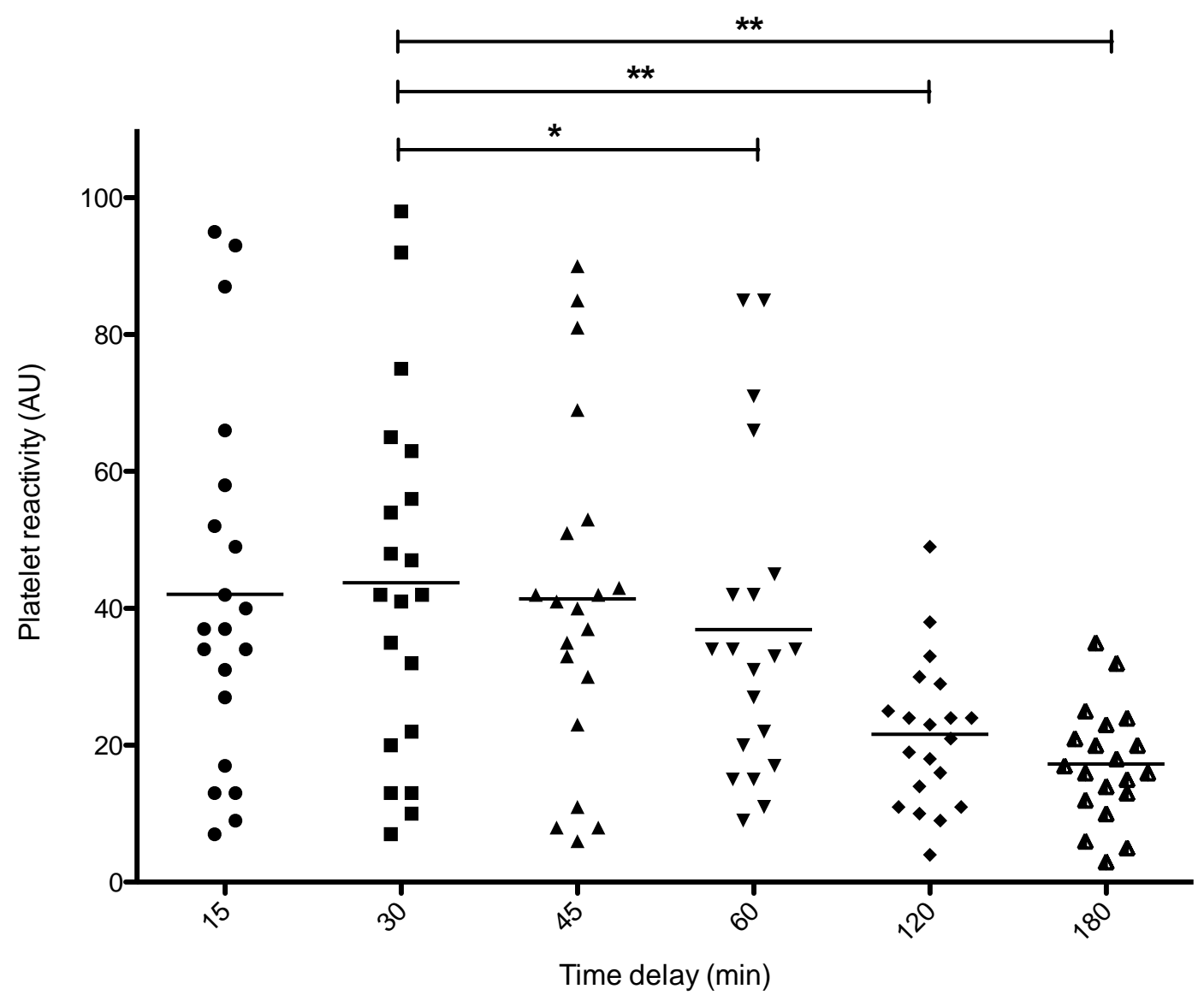

Figure 2-7 Effect of time delay from collection to assay performance on platelet aggregation.

Platelet aggregation measured in hirudin-treated blood at 15, 30, 45, 60, 120 and 180 minutes post sampling. ${ }^{*} \mathrm{P}<0.05,{ }^{* *} \mathrm{P}<0.001$. 


\subsubsection{Manual Pipetting}

The CV for 20 samples anticoagulated with hirudin and analysed using the modified Multiplate ${ }^{\circledR}$ protocol with a calibrated manual pipette are shown in Figure 2-8. The mean CV was $9.2 \%(95 \% \mathrm{Cl}, 6.1-12.1)$ with the use of a manual pipette. This value did not differ significantly from the mean CV observed using the Multiplate automated pipette $(P=0.55)$.

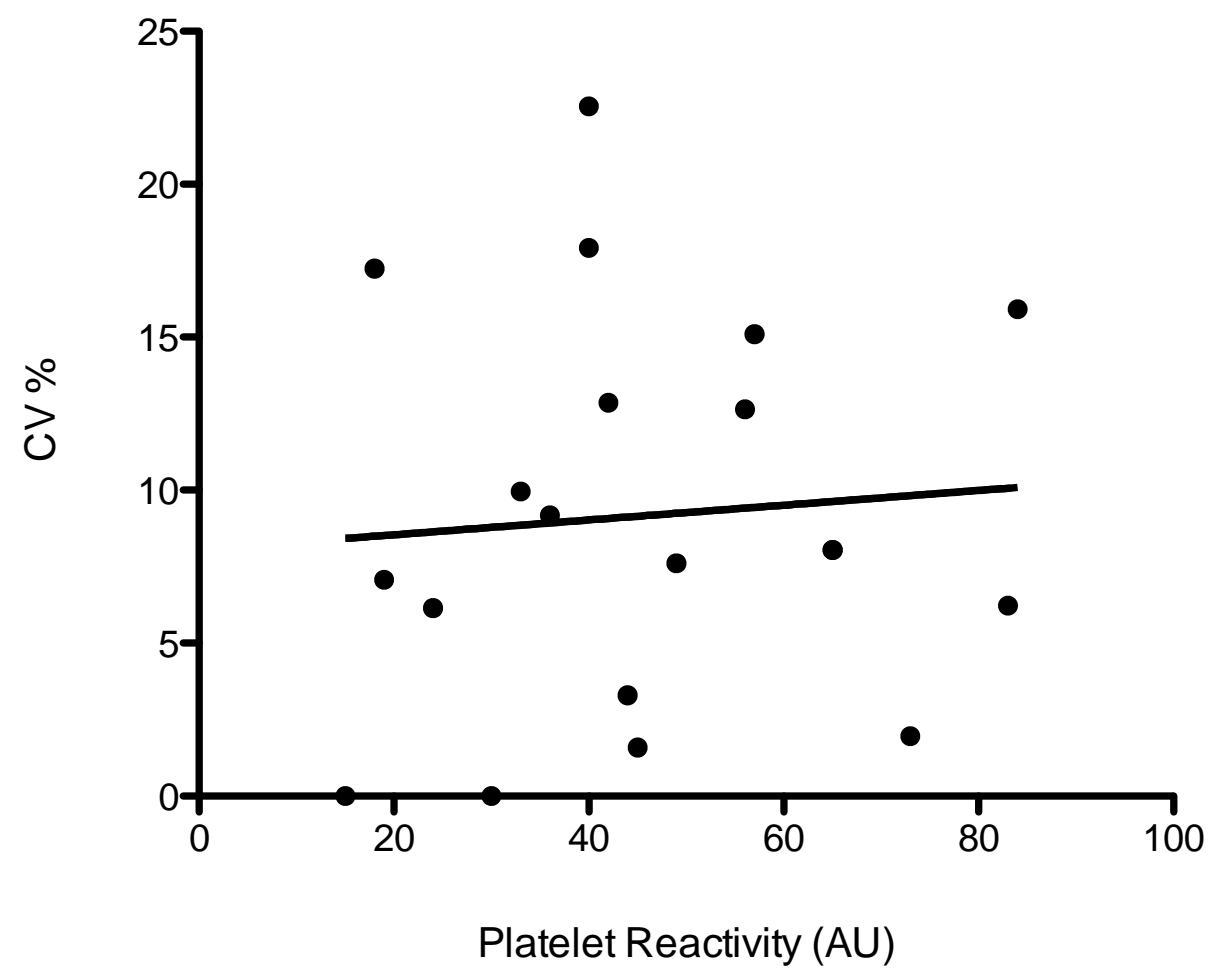

Figure 2-8 Coefficient of variance (CV) in platelet aggregation using calibrated manual pipette and ADP stimulation

The CV calculated by the standard deviation divided by the mean of the two tests from the same patient sample was expressed as $\mathrm{CV} \%$ and plotted against absolute platelet reactivity (AU). There was no correlation between increased platelet reactivity and variance $\left(r^{2} 0.006\right)$. Each point represents duplicate patient samples $(n=20)$. 


\subsection{Discussion}

Although there have been studies looking at different pre analytical factors that may influence platelet aggregation and the analytical precision of the Multiplate assay, this is the first study to bring all of these factors together and evaluate ADP and AA dependent platelet aggregation in ACS patients on dual antiplatelet therapy. We have found an inter-assay mean coefficient of variation of $10.8 \%$ with ADP stimulation and $11 \%$ with AA stimulation using the manufacturers recommended methodology. The assessment of platelet aggregation was influenced by time of assessment and the type of anticoagulant used. Use of manual rather than automated pipette was not associated with a significant decrease in the mean coefficient of variation

Several other studies have reported the CV using the Multiplate system. In groups of healthy volunteers coefficients of variation ranging from $3.9 \%$ to $14.9 \%$ have been reported (290-295). Coefficients of variation for the Multiplate system in ACS patients on dual antiplatelet therapy of $5.8 \%$ and $6.2 \%$ were also reported in two of these studies $(290,292)$. However, hirudin was used as the anticoagulant in only one of these studies (294), and many studies were performed with a very small number of subjects or did not outline their methodology in detail. Our measurement of mean CV for $A D P$ at $10.8 \%$ and $11 \%$ with $A A$ is within the range of previous studies, and has been determined from a significantly larger patient group.

It is possible that a small variation in the quantity of ADP delivered, leading to variation in the final concentration of ADP within the test cell, contributes to the analytical imprecision of the assay. Because the automated pipette in the Multiplate ${ }^{\circledR}$ system delivers volumes over a wide range $(20 \mu \mathrm{L}$ to $300 \mu \mathrm{L})$, we believed that the use of a calibrated $100 \mu \mathrm{L}$ manual pipette would reduce any variation in delivered ADP. However, the use of a manual pipette did not lead to a significant reduction in the CV. Thus the analytical precision of the assay is not significantly influenced by the use of the $300 \mu \mathrm{L}$-automated pipette.

The agonist $A A$ is relatively unstable and the manufacturer's guidelines recommend a one time thawing and stability for only 24 hours at room temperature. It is possible 
that a small amount of variation in the analytical precision could be due to the decreasing stability of the AA agonist over time. However, the use of this assay was tightly controlled and fresh AA was used daily.

The Multiplate records both sets of electrode pairs within the same test cell. This acts as an internal quality check (IQC) that the test cell is functioning appropriately and if there is a difference of more than $20 \%$ between the two tests, the user is asked to repeat the measurement. We found that an increase in the IQC did not correlate with an increase in the CV for ADP or AA induced platelet reactivity and did not differ when measuring different levels of platelet reactivity (data not shown). Many of the other platelet function assays do not offer internal quality assessments or visual checks such as platelet aggregation tracings to ensure the measurement is acceptable

The Multiplate is a simple relatively inexpensive point of care assay, which has a flexible platform to measure several clinically relevant pathways of platelet activation. The level of reproducibility of the assay reported here is slightly higher than has been reported using VerifyNow (6\%) (195), or light transmission aggregometry (LTA) (6.8\%) (296) which is considered to be the gold standard of platelet function testing but is complex and laborious. Despite this level of inter-assay variability, measurement of platelet aggregation using the Multiplate assay correlated well with the clinical endpoints of increased stent thrombosis and bleeding risk (278) (297). It has also been proposed that the Multiplate offers a more physiological representation of platelet reactivity in vivo. As the test requires strong interactions between platelets and thrombin in whole blood to maintain connections and remain attached to the electrodes, this reactivity is closer to the response of platelets seen in the body, such as attaching to an injured vascular wall or atherosclerotic plaque. The LTA assay requires isolated platelets to aggregate in a liquid phase and the VerifyNow system provides fibrinogen-coated beads for platelets to attach to, which is further removed from the in vivo setting.

In this study we found the anticoagulant tube used to collect blood had a significant effect on platelet aggregation. Hirudin is the anticoagulant recommended for use with the Multiplate analyser by the manufacturer. Hirudin is a direct thrombin inhibitor and 
has the advantage of allowing analysis of platelet function under physiological calcium concentrations. By contrast, anticoagulation with the calcium-chelating agent sodium citrate reduces free plasma and cytosolic calcium levels and has been shown to reduce platelet activation and aggregation as measured by other methods $(298,299)$. It is therefore not surprising that anticoagulation with sodium citrate in our study reduced platelet aggregation by $31 \%$ when compared to hirudin and the addition of $\mathrm{CaCl}_{2}$ resulted in levels of platelet aggregation similar to those seen in hirudin-treated blood. Other studies in healthy volunteers have reported similar findings $(295,300)$. Anticoagulation with heparin does not affect calcium concentrations but does cause platelet activation (301-304). Consistent with this, treatment with heparin in our study resulted in a $65 \%$ increase in residual platelet aggregation. Our findings suggest that it is important to standardise the anticoagulant used and for the reasons stated above we would recommend using hirudin.

For platelet function testing to be effective, the results need to be obtained quickly and easily. The Multiplate assay delivers results within 10 minutes and the manufacturer recommends testing 30 to 180 minutes post-sampling. In this study we found platelet aggregation varied significantly over the 180-minute testing time with reduced platelet aggregation at later time points. Therefore the manufacturer's guideline for testing over a period of 180 minutes is not recommended and would increase variance in measurement. We would suggest that the assay should be performed within 15 to 45 minutes of blood sampling. The mechanisms responsible for the change in reactivity over time ex-vivo, are likely be multifactorial and are poorly defined at present.

\subsection{Limitations}

To test the CV of ADP and AA induced platelet reactivity we measured 30 patients with 2 replicates in each person, and 20 measurements with single replicates for the other testing parameters. With a sample size of 30 we had modest $95 \%$ confidence intervals (CI) of $7.5 \%$ to $14.1 \% \mathrm{CV}$ with ADP and $8.3 \%$ to $15.3 \% \mathrm{CV}$ with AA stimulated blood. If we had tested more individuals we could have reduced the $95 \% \mathrm{Cl}$ of our calculated CVs and increased our confidence in the CV of the assay. However, every test has a 
consumable cost of approximately $\$ 17$ so the addition of extra measurements would have incurred a much greater cost. A CV of between $7.5 \%$ and $14 \%$ is acceptable and additional features such as the internal quality check makes it practical to use in clinical studies. Similarly, with a sample size of 20 we had the power to detect a difference of $15 \%$ in platelet reactivity between time points and anticoagulant tubes at a significance level of 0.05 . By increasing the sample size, we could have more power to detect a smaller difference between time points or anticoagulant tubes. However, this level of power allowed us to determine a time point and anticoagulant that can be standardised and therefore reduce some of the variance in the assay.

From this study we could not determine which time point or anticoagulant is the 'best' or optimal one to use. We can however, pick a time point of 30 minutes and the manufacturers guide of hirudin anticoagulated tubes to use in the rest of our studies, with a known level of standard error and both are practical for testing in a clinical setting.

\subsection{Conclusion}

Pre-analytical and analytical variables affect the assessment of ADP and AA-induced platelet aggregation with the Multiplate assay. This study highlights the importance of standardising these methodological elements to reduce variation. Therefore, a time delay of 30 minutes post sampling, hirudin anticoagulated tubes for blood sampling and use of the automated pipette will be standardised throughout the rest of the thesis to measure ADP and AA induced platelet aggregation. Despite alternative platelet function assays having lower CVs, the Multiplate is more economical, flexible and easy to use. These factors along with a moderate CV of $10-11 \%$, provides a reasonable system to test platelet reactivity in clinical studies. 


\section{Chapter 3 - Platelet reactivity in Acute Coronary Syndromes}




\subsection{Introduction}

Multiple studies have demonstrated a clear association between a suboptimal response to clopidogrel and an increased risk of cardiovascular events in patients with ACS or undergoing $\mathrm{PCl}(238,279)$. The majority of these studies have been conducted in North American and European populations with minor or no inclusion of Maori, Pacific Islander or Asian ethnicities. The genetic make up of a NZ ACS population differs substantially from international trials, in that it has a considerable portion of Maori and Pacific Islanders. Previous studies have suggested that ethnicity has a significant impact on the response to clopidogrel $(305,306)$ and therefore collection of local data is important to identify patients at increased risk.

The treatment of HOTPR with subsequent loading doses of clopidogrel or increased maintenance doses have been ineffective at significantly lowering residual platelet reactivity in a large proportion of the patients treated. New P2Y12 inhibitors such as prasugrel have demonstrated a more potent and consistent inhibition of platelets than clopidogrel (307). The TRITON-TIMI 38 trial compared clopidogrel to prasugrel in ACS patients undergoing $\mathrm{PCl}$ and found that use of prasugrel resulted in a reduction in ischaemic events but this was at the cost of an increased risk of bleeding [10]. The utility of prasugrel in patients with HOTPR has not been examined. There are a number of different methodological approaches to assess platelet reactivity including: VerifyNow, light transmission aggregometry, VASP and the Multiplate as outlined in the previous chapter.

The aims of this study were:

1. To determine the incidence of HOTPR in a NZ ACS population

2. To investigate the presence of clinical predictors of HOTPR

3. To examine the effectiveness of prasugrel in reducing platelet reactivity in those patients with HOTPR. 


\subsection{Methods}

\subsubsection{Study Population:}

Patients presenting to Wellington Regional Hospital with ACS between October 2010 and March 2011 were eligible for inclusion in the study if there was an invasive approach (coronary angiography $\pm \mathrm{PCl}$ ) planned and they were adequately pretreated with aspirin and clopidogrel. An ACS was defined as symptoms suggestive of myocardial ischaemia lasting $>10$ minutes and either troponin elevation or $\geq 1 \mathrm{~mm}$ of new ST segment deviation or T wave inversion on an electrocardiogram in at least 2 contiguous leads. Adequate pretreatment was defined as chronic therapy with aspirin ( $\geq 75 \mathrm{mg}$ ) and clopidogrel ( $\geq 75 \mathrm{mg}$ ) and / or loading with aspirin $\geq 300 \mathrm{mg}$ at least 2 hours and clopidogrel $\geq 300 \mathrm{mg}$ at least 6 hours or prior to enrolment. This time point is based on the average time to achieve peak platelet inhibition in patients receiving clopidogrel (309). Exclusion criteria included a platelet count less than $100 \times 10^{9} / \mathrm{L}$, known platelet function disorder, administration of a fibrinolytic agent within 24 hours of enrolment or administration of a glycoprotein $\mathrm{Ilb} / \mathrm{lll}$ la receptor antagonist within a week prior to enrolment. The study was reviewed and approved by the Central Regional Ethics Committee.

\subsubsection{Data Collection}

Patient demographics, clinical characteristics, medications including antiplatelet therapy, clinical management, procedural variables and in-hospital outcomes were obtained prospectively from review of the medical records and cardiac catheterization database. Ethnicity was self-identified by the patient. All aspects of clinical management, including prescription of antiplatelet therapy, were at the discretion of the attending physicians.

\subsubsection{Blood Collection and Platelet Function testing}

Blood for platelet function testing was collected and testing as described in section 2.2.2. Patients that were identified as having HOTPR were presented to the consultant 
cardiologist performing the angiogram and it was their decision whether the patient received prasugrel. Contraindications to prasugrel were; weight under $65 \mathrm{~kg}$, age over 75 and renal dysfunction classified as an eGFR<45. Where patients with HOTPR were treated with prasugrel a further blood sample for measurement of platelet reactivity was taken at least 2 hours post administration of a $60 \mathrm{mg}$ loading dose unless a glycoprotein Ilb/Illa receptor antagonist had been administered. Cardiac enzymes including high sensitivity Troponin T (hs-TnT) and electrocardiograms were routinely performed prior to cardiac catheterization and following $\mathrm{PCl}$.

\subsubsection{Definitions}

The clopidogrel dose was defined as "high" if patients had received a $600 \mathrm{mg}$ loading dose followed by a $150 \mathrm{mg}$ daily maintenance dose. Intermediate dose was defined as either a $600 \mathrm{mg}$ loading dose followed by a $75 \mathrm{mg}$ daily maintenance dose or a $300 \mathrm{mg}$ loading dose coupled with a $150 \mathrm{mg}$ maintenance dose. Low dose was defined as a $300 \mathrm{mg}$ loading dose followed by a $75 \mathrm{mg}$ daily maintenance dose or chronic therapy with $75 \mathrm{mg}$ daily of clopidogrel. HOTPR was defined as $>47 \mathrm{AU}$ and a cutoff value of $<18 \mathrm{AU}$ was used to define an enhanced response to clopidogrel. These cutoff values have been determined from a comparative analysis of the risk for bleeding and stent thrombosis across different levels of $\mathrm{P}_{2} \mathrm{Y}_{12}$ receptor inhibition (310). A periprocedural enzyme rise was defined as an increase in hs-TnT to > three times the upper reference limit (>39ng/L) for those with preprocedural hs-TnT levels within the normal range. In those with elevated preprocedural hs-TnT that were stable or falling a further elevation of hs-TnT >39 $\mathrm{ng} / \mathrm{L}$ was required.

\subsubsection{Statistical Analysis}

A power calculation was performed based on a total population of 250 patients and HOTPR rate of around $33 \%$ based on the literature. In a population of 250 ACS patients we expected approximately 200 Europeans, 35 Maori and Pacific Islanders and 15 other ethnicities based on the demographics of our referral population and catchment area. The confidence intervals for these populations were 6.52 for Europeans, 15.53 
for Maori and Pacific Islanders and 23.8 for other ethnicities. Therefore we had the power to detect a difference of $20 \%$ or more in the rate of HOTPR between Europeans and Maori and Pacific Islanders.

Continuous variables are expressed as median and interquartile range (IQR). Categorical variables are expressed as frequencies and percentages. We compared the proportion of patients with HOTPR by diabetes, ethnicity and dose group using Chi-Squared test. Absolute values for residual platelet reactivity (AU) were compared by ethnicity, diabetes and dose groups using the Kruskal Wallis test. The relationship between body mass index (BMI) and residual platelet reactivity was assessed by Spearman's correlation coefficient ( $r h o$ ). Residual platelet reactivity before and after treatment with prasugrel was compared using Wilcoxon Signed rank test. All statistical tests were performed using PASW 18.0 (IBM, NY, USA).

\subsection{Results}

\subsubsection{Baseline demographics and prevalence of HOTPR}

During the study 250 patients with ACS met the inclusion criteria and were enrolled in the study. Their baseline demographics, clinical characteristics and laboratory data are shown in Table 3-1. The median age was 62 (54-72) years with $74.8 \%$ being male and $20.8 \%$ having diabetes. STEMI was the presentation in $28.4 \%$, NSTEMI in $62.4 \%$ and unstable angina in $9.2 \%$. The majority identified themselves as European $81.2 \%$, a further $14 \%$ as Maori or Pacific Islanders and $4.8 \%$ as other ethnicities. Platelet reactivity was measured in all 250 patients with the Multiplate analyser prior to cardiac catherterisation. Of the 250 patients tested, 95 patients (38\%) had HOTPR. 
Table 3-1 Patient baseline demographics, clinical characteristics and laboratory data

\begin{tabular}{|c|c|c|c|c|}
\hline & $\mathrm{N}=250$ & $\begin{array}{l}\text { HOTPR } \\
(\mathrm{N}=95)\end{array}$ & $\begin{array}{l}\text { NO HOTPR } \\
(\mathrm{N}=155)\end{array}$ & $P$ value \\
\hline Age (years) & 62 (54-72) & $62.0(55-71)$ & $61.0(53-72)$ & 0.679 \\
\hline Male, n (\%) & $187(74.8)$ & $73(76.8)$ & $114(73.5)$ & 0.560 \\
\hline $\mathrm{BMI}$ & $28(19-56)$ & $29(26-33)$ & $27(25-31)$ & 0.066 \\
\hline \multicolumn{5}{|l|}{ Ethnicity, n (\%) } \\
\hline European & $203(81.2)$ & $71(74.7)$ & $132(85.2)$ & 0.041 \\
\hline $\begin{array}{l}\text { Maori and Pacific } \\
\text { Islander }\end{array}$ & $35(14)$ & $20(21.1)$ & $15(9.7)$ & 0.019 \\
\hline Other & $12(4.8)$ & $4(4.2)$ & $8(5.2)$ & 0.649 \\
\hline \multicolumn{5}{|l|}{ Risk Factors, n (\%) } \\
\hline Hypertension & $145(58.0)$ & $55(57.9)$ & $90(58.1)$ & 0.979 \\
\hline Dyslipidaemia & $137(54.8)$ & $53(55.7)$ & $84(54.2)$ & 0.806 \\
\hline Diabetes & $52(20.8)$ & $26(27.3)$ & $26(16.7)$ & 0.045 \\
\hline Current Smoker & $65(26.0)$ & $27(28.4)$ & $38(24.5)$ & 0.494 \\
\hline \multicolumn{5}{|l|}{ Medical History, n (\%) } \\
\hline Previous MI & $56(22.4)$ & $26(27.4)$ & $30(19.4)$ & 0.140 \\
\hline Previous PCl & $40(16.0)$ & $21(22.1)$ & $19(12.3)$ & 0.039 \\
\hline Previous CABG & $20(8.0)$ & $9(9.5)$ & $11(7.1)$ & 0.501 \\
\hline \multicolumn{5}{|l|}{$\begin{array}{l}\text { Clinical Presentation, } n \\
(\%)\end{array}$} \\
\hline STEMI & $71(28.4)$ & $28(29.5)$ & $43(27.8)$ & 0.768 \\
\hline NSTEMI & $156(62.4)$ & $58(61.1)$ & 98 (63.2) & 0.731 \\
\hline Unstable Angina & $23(9.2)$ & $9(9.5)$ & $14(9.1)$ & 0.919 \\
\hline \multicolumn{5}{|l|}{ Laboratory data } \\
\hline Creatinine (umol/L) & $88(76-102)$ & $89(77-102)$ & $88(74-102)$ & 0.357 \\
\hline $\begin{array}{l}\text { Platelet Count } \\
\left(10^{9} / \mathrm{L}\right)\end{array}$ & $\begin{array}{l}220(187- \\
267)\end{array}$ & $\begin{array}{l}223(190- \\
278)\end{array}$ & $\begin{array}{l}217(187- \\
264)\end{array}$ & 0.135 \\
\hline $\begin{array}{l}\text { PPI use, n (\%) } \\
\text { Clopidogrel Dosing } \\
\text { High } \\
\text { Intermediate } \\
\text { Low }\end{array}$ & $\begin{array}{l}65(26.0) \\
41(16.4) \\
55(22.0) \\
154(61.6) \\
\end{array}$ & $\begin{array}{l}22(23.2) \\
11(11.6) \\
14(14.7) \\
70(73.7) \\
\end{array}$ & $\begin{array}{l}4(27.7) \\
30(19.4) \\
41(26.5) \\
84(54.2)\end{array}$ & $\begin{array}{l}0.423 \\
0.107 \\
0.30 \\
0.003\end{array}$ \\
\hline
\end{tabular}




\subsubsection{Predictors of HOTPR}

To investigate the predictors of HOTPR, baseline demographics, clinical characteristics and laboratory data were tested against rates of HOTPR in a univariate analysis. Ethnicity, diabetes and clopidogrel dosing were found to be predictors of HOTPR. Maori and Pacific Islanders had higher residual platelet reactivity (50 AU (35-70) versus $38 \mathrm{AU}(250-570), \mathrm{p}=0.014)$ and a higher rate of HOTPR compared to Europeans (57\% versus $35.9 \%, p=0.013$, Table $3-2$ and Figure $3-1$ ).

Table 3-2 Patient Ethnicity and platelet reactivity

\begin{tabular}{|l|l|l|l|}
\hline & $\begin{array}{l}\text { Maori and Polynesian } \\
(35)\end{array}$ & European (203) & P value \\
\hline HOTPR (\%) & $20(57.0)$ & $71(35.9)$ & 0.013 \\
\hline Platelet reactivity (AU) & $50(34-77)$ & $38(25-57)$ & 0.014 \\
\hline
\end{tabular}

A

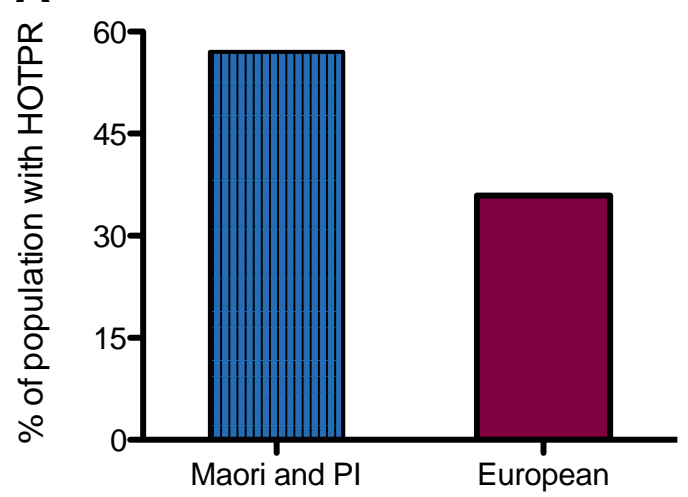

B

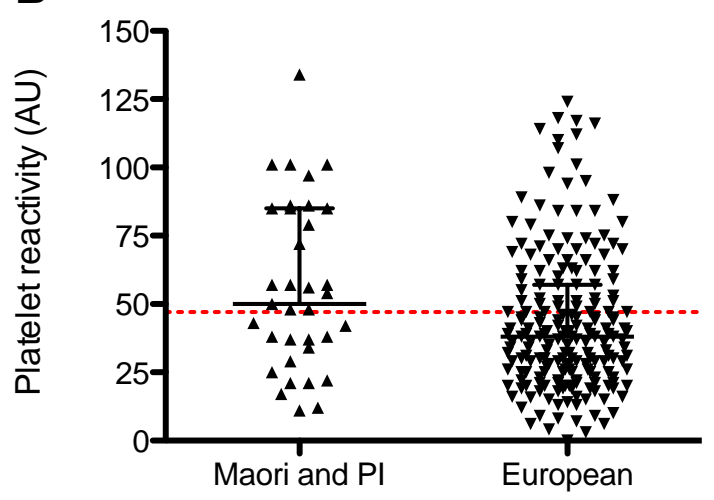

Figure 3-1 Patient ethnicity and platelet reactivity.

The rate of HOTPR $(A)$ analysed by chi-square test $(p=0.013)$ and the absolute levels of platelet reactivity (B) analysed by Kruskal Wallis test $(p=0.01)$ in Maori and Pacific Islanders compared to Europeans. The red dotted line represents the 47 AU HOTPR cut-point. Median and IQR absolute platelet reactivity are displayed. 
Patients with diabetes were also found to have higher residual platelet reactivity (43 $\mathrm{AU}(29-77)$ versus $38 \mathrm{AU}(25-55), \mathrm{p}=0.029)$ and higher rates of HOTPR compared to non-diabetics (50\% versus 34.8\%, $p=0.045$, Table 3-3 and Figure 3-2).

Table 3-3 Diabetes and platelet reactivity

\begin{tabular}{|l|l|l|l|}
\hline & Diabetic (52) & Non-Diabetic (198) & P Value \\
\hline HOTPR (\%) & $26(50.0)$ & $69(34.8)$ & 0.045 \\
\hline Platelet reactivity (AU) & $43(29-77)$ & $38(25-55)$ & 0.029 \\
\hline
\end{tabular}

A

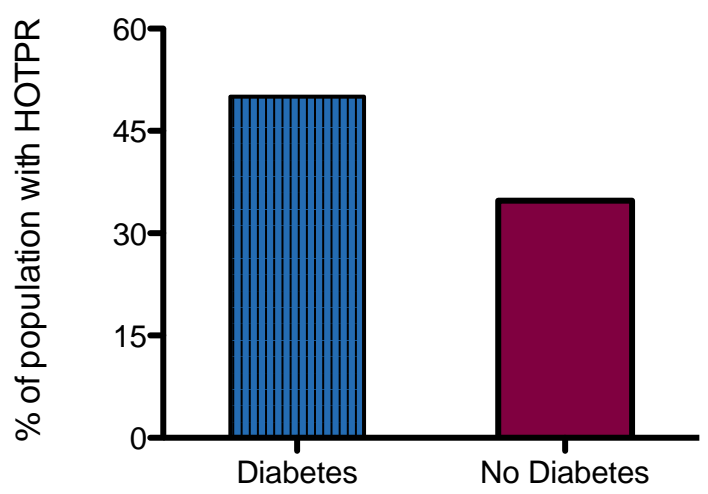

B

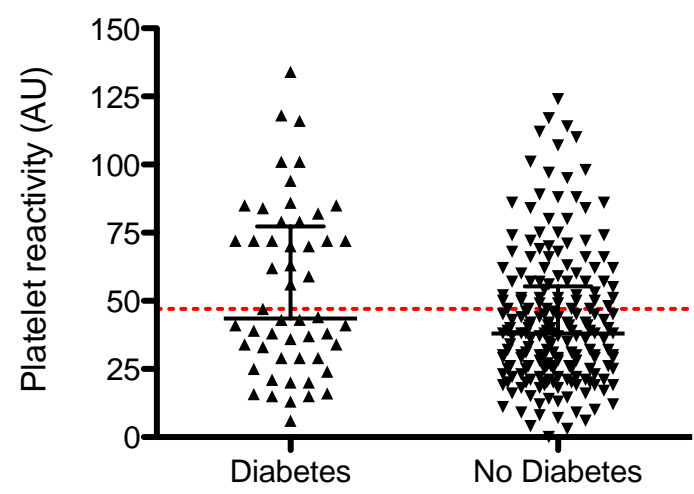

Figure 3-2 Diabetes and platelet reactivity

The rate of HOTPR (A) analysed by chi-square test $(p=0.045)$ and the absolute levels of platelet reactivity (B) analysed by Kruskal Wallis test $(p=0.029)$ in diabetics and non-diabetics. The red dotted line represents the 47 AU HOTPR cut-point. Median and IQR absolute platelet reactivity are displayed.

The rate of diabetes was higher in Maori and Pacific Islanders compared to Europeans (38.8\% versus $15.3 \%, p=0.001)$. Maori and Pacific Islanders with diabetes also had the highest absolute levels of platelet reactivity 75AU (41 - 90) versus European diabetics $40 \mathrm{AU}(23$ - 70), non-diabetic Maori and Pacific Islanders $40 \mathrm{AU}(24-56)$ and non-diabetic Europeans (38 AU (25 - 54), p = 0.004, Table 3-4, Figure 3-3B). The rates of HOTPR displayed a similar trend with the highest in Maori and Pacific Islander diabetics (71\%), followed by European diabetics (42\%), non-diabetic Maori and Pacific Islanders (45\%) and non-diabetic Europeans (33\%, $p=0.03$, Table 3-4, Figure 3-3A). 
Table 3-4 Diabetes, ethnicity and platelet reactivity

\begin{tabular}{|l|l|l|l|l|l|}
\hline & $\begin{array}{l}\text { Diabetes and } \\
\text { Maori and PI } \\
(14)\end{array}$ & $\begin{array}{l}\text { Diabetes and } \\
\text { European (38) }\end{array}$ & $\begin{array}{l}\text { No Diabetes } \\
\text { and Maori } \\
\text { and PI (22) }\end{array}$ & $\begin{array}{l}\text { No Diabetes } \\
\text { European } \\
(176)\end{array}$ & P value \\
\hline HOTPR (\%) & $10(71)$ & $16(42)$ & $10(45)$ & $59(33)$ & 0.03 \\
\hline $\begin{array}{l}\text { Platelet } \\
\text { reactivity (AU) }\end{array}$ & $75(41-90)$ & $40(23-70)$ & $40(24-56)$ & $38(25-54)$ & 0.004 \\
\hline
\end{tabular}

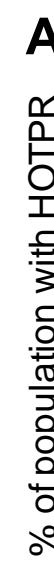

A

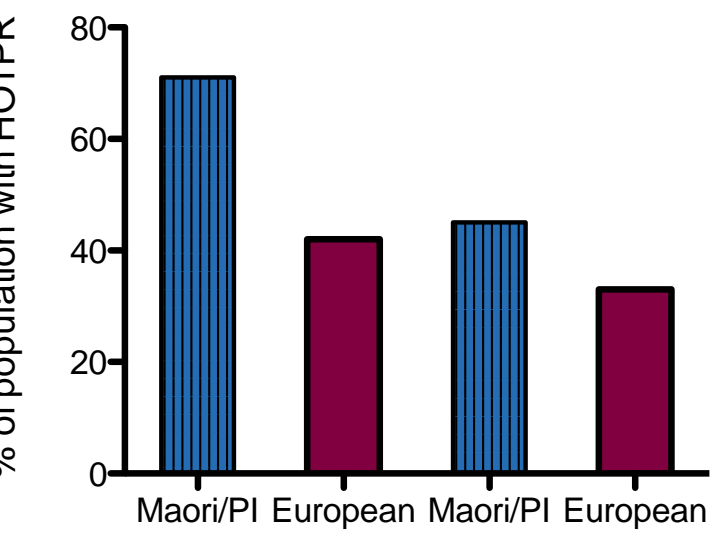

Diabetes

No Diabetes

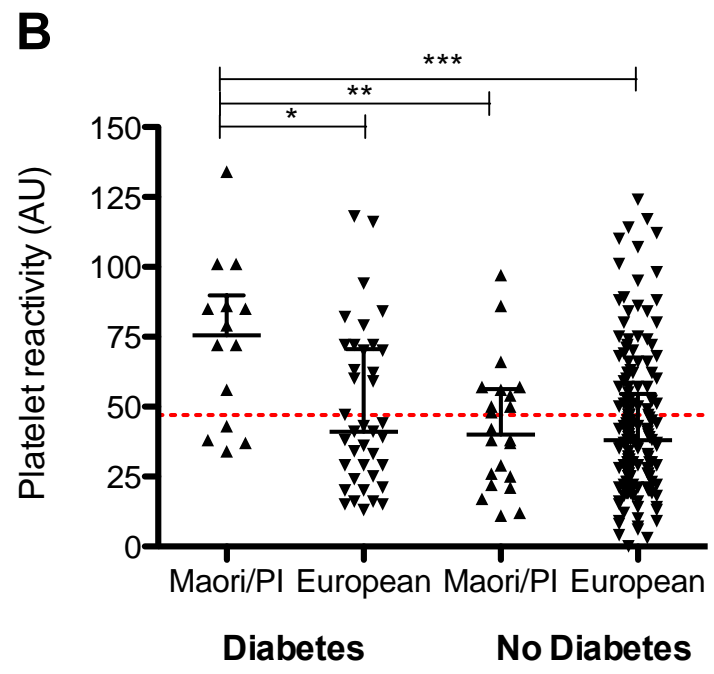

Figure 3-3 Diabetes, ethnicity and platelet reactivity

The rate of HOTPR $(A)$ analysed by one way ANOVA $(p=0.004)$ and the absolute levels of platelet reactivity (B) analysed by one way ANOVA $(p<0.05)$ and Kruskal Wallis test ${ }^{*} p<0.05$, ${ }^{* *} p<0.01,{ }^{* * *} p<0.001$ in Maori and Pacific Islanders compared to Europeans with and without diabetes. The red dotted line represents the 47 AU HOTPR cut-point. Median and IQR absolute platelet reactivity are displayed.

Although all patients were pre-treated with aspirin and clopidogrel, a variety of clopidogrel dosing regimens were used with the majority of patients $(61.6 \%)$ being treated with a low dose regimen. To understand the relationship between clopidogrel dose and residual platelet reactivity, we compared the rates of HOTPR in patients who received low, intermediate, and high dose regimens (Table 3-5). Our findings show that patients treated with a low dose regimen had significantly higher rates of HOTPR compared to those treated with intermediate or high dose regimens $(p=0.009$, Figure 3-4A). Additionally, the mean residual platelet reactivity decreased with increasing clopidogrel dose $(p=0.007$, Figure $3-4 B)$. There was no significant correlation between $\mathrm{BMI}$ and residual platelet reactivity (rho $=0.1, \mathrm{p}=0.1$ ). Mean residual platelet reactivity and rates of HOTPR were also not affected by concomitant treatment with a proton pump inhibitor or the type of ACS at presentation. 
Table 3-5 Clopidogrel dose and platelet reactivity

\begin{tabular}{|l|l|l|l|l|}
\hline Clopidogrel Dose & Low (154) & $\begin{array}{l}\text { Intermediate } \\
(55)\end{array}$ & High (41) & P value \\
\hline HOTPR (\%) & $70(45.4)$ & $14(25.4)$ & $11(26.8)$ & 0.009 \\
\hline $\begin{array}{l}\text { Platelet reactivity } \\
\text { (AU) }\end{array}$ & $43(30-66)$ & $37(22-49)$ & $29(20-52)$ & 0.007 \\
\hline
\end{tabular}

A

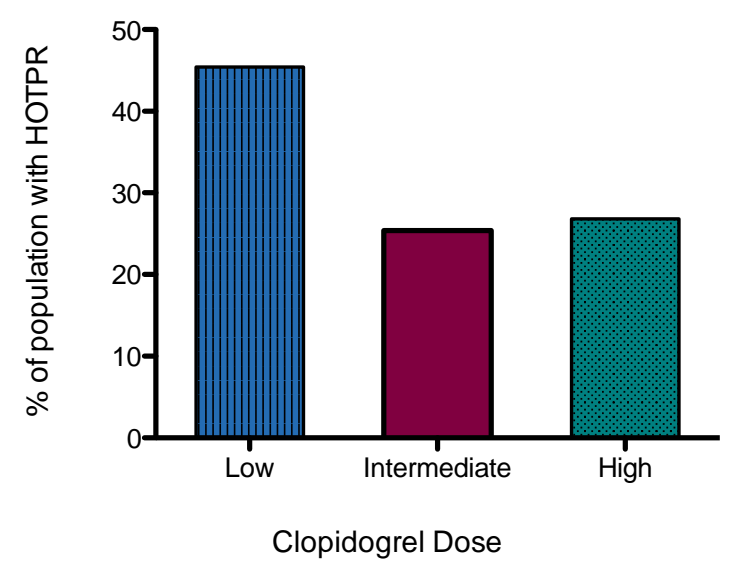

B

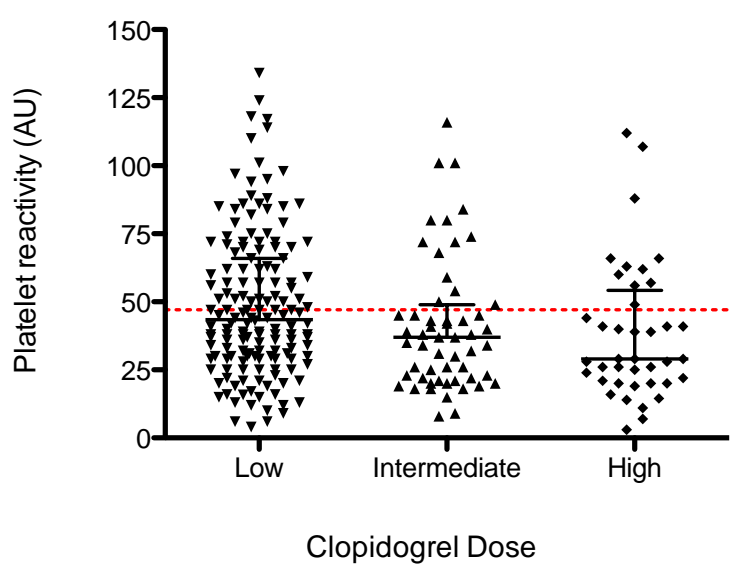

Figure 3-4 Clopidogrel dose and platelet reactivity.

The rate of HOTPR $(A)$ analysed by one-way ANOVA $(p=0.009)$ and the absolute levels of platelet reactivity $(B)$ analysed by one-way ANOVA $(p=0.007)$ in patients receiving low, intermediate and high doses of clopidogrel. The red dotted line represents the 47 AU HOTPR cut-point. Median and IQR absolute platelet reactivity are displayed. 


\subsubsection{Effectiveness of prasugrel on HOTPR}

Of the 95 patients with HOTPR, 29 patients were treated with prasugrel and underwent platelet function testing at least 2 hours after loading with 60mg. The remaining 66 patients did not receive prasugrel due to contraindications or because they were being medically managed, undergoing $C A B G$, received glycoprotein $\mathrm{Ilb} / \mathrm{Illa}$ inhibitors during their angiogram or the managing clinician chose not to use prasugrel. Residual platelet reactivity was lowered markedly by prasugrel treatment (66 AU (5677) before versus $23 \mathrm{AU}(11-34)$ after, $p<0.001)$. Residual platelet reactivity was lowered below the HOTPR cut-point in 26 (89.7\%) patients. The 3 patients whose reactivity remained above the $47 \mathrm{AU}$ cut-point still had a dramatic lowering of residual platelet reactivity following prasugrel administration (Figure 3-5). In 10 patients (34.5\%), treatment with prasugrel resulted in a platelet reactivity level below the cutpoint defining an enhanced response.

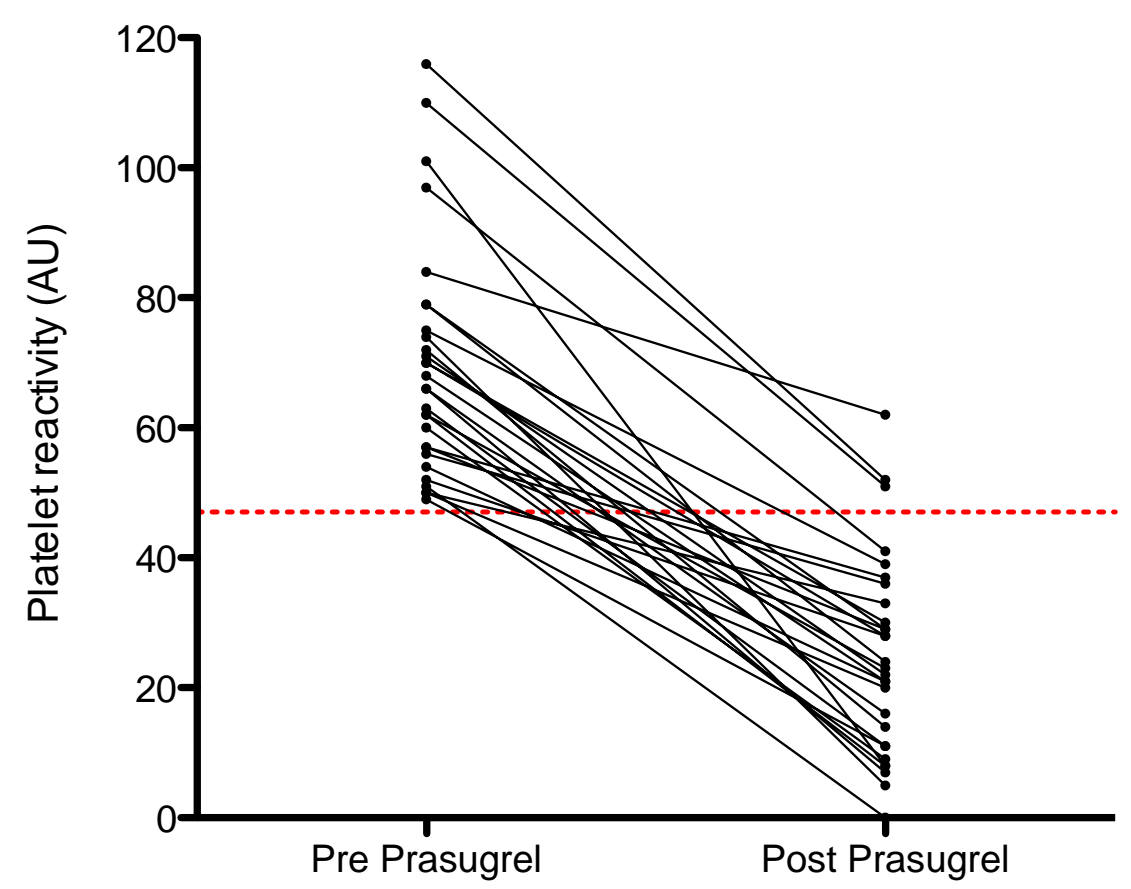

Figure 3-5 Platelet reactivity pre and post prasugrel administration

Platelet reactivity $(\mathrm{AU})$ of patients with HOTPR pre and post $60 \mathrm{mg}$ prasugrel loading. The red dotted line represents the 47 AU HOTPR cut point.

All patients involved in the study underwent coronary angiography. Of these 42 (16.8\%) had minor coronary disease, 99 (39.6\%) single vessel disease, 63 (25.2\%) 2 
vessel disease and 46 (18.4\%) 3 vessel disease. Sixty-nine patients (27.6\%) were managed medically, 154 (61.6\%) treated with $\mathrm{PCl}$ and 27 (10.8\%) referred for CABG. In-hospital there were no cases of death, stent thrombosis, target vessel revascularisation or major bleeding. In the patients undergoing $\mathrm{PCl}$, periprocedural enzyme rises occurred in 32 (20.8\%), while clinical periprocedural myocardial infarction occurred in 7 (2.8\%). Periprocedural enzyme elevation occurred in 13 (26.5\%) of those with HOTPR compared to 19 (18.3\%) without HOTPR $p=0.23 .3 .4$

\subsection{Discussion}

Our study is the first to look at the frequency of HOTPR following treatment with clopidogrel in a NZ population. HOTPR was common in our population and importantly there was an ethnic disparity with Maori and Pacific Islanders having a much higher rate of HOTPR. Diabetes was also associated with higher rates of HOTPR. Although treatment with high dose clopidogrel was associated with a reduction in residual platelet reactivity, $26 \%$ of patients treated with the high dose regimen still had HOTPR. To our knowledge this study was the first to examine the effect of prasugrel on platelet reactivity in ACS patients with HOTPR. We found that prasugrel therapy in those with HOTPR resulted in a consistent and marked further reduction in platelet reactivity

The current study is the first to include Maori and Pacific Islanders in significant numbers. Both of these ethnic groups have disproportionately high rates of cardiovascular disease and adverse outcomes following cardiovascular events (306). The very frequent occurrence of HOTPR despite adequate clopidogrel therapy in this group may contribute to their poor outcomes following cardiovascular events and is a potential target for intervention with more potent antiplatelet agents.

One contributing factor to the observed high rates of HOTPR in Maori and Pacific Islanders may be the increased carriage of loss of function SNPs in the CYP2C19 gene [15]. These SNPs have been shown to result in reduced formation of clopidogrel's active metabolite, reduced platelet inhibition and an increased risk of thrombotic events following $\mathrm{PCl}(306)$. The most frequent and important of these polymorphisms is the CYP2C19*2. It has been previously demonstrated that the allele CYP2C19*2 
occurs more frequently in Maori (24\%) compared to NZ Europeans (15\%)(230). Another polymorphism CYP2C19*3 that results in reduced function also occurs more frequently in Maori (1.8\%) than Europeans (0.4\%)(230). These findings suggest that CYP2C19 polymorphisms may be contributing to the phenotypic differences found in this study. However, it is likely that multiple genetic and environmental factors play a role. Previous studies have suggested that CYP2C19 loss of function polymorphisms only account for a small minority of the inter-individual variability in the response to clopidogrel therapy found in other populations $(236,309)$.

Patients with diabetes mellitus are at high risk for recurrent cardiovascular events after an ACS (246). Multiple studies have suggested that platelets of diabetic patients are larger, hyper-reactive and show increased adhesion and aggregation $(250,255)$. It is therefore not surprising that in our study we observed a higher rate of HOTPR in patients with diabetes mellitus. This finding is also consistent with those of a number of other studies that have also demonstrated increased rates of HOTPR in diabetic patients using different platelet function tests (310-312). The combination of increased platelet reactivity and reduced responsiveness to clopidogrel may explain the increased benefit seen in randomized trials with more potent antiplatelet agents, such as prasugrel and glycoprotein IIb/IIla inhibitors in diabetic patients $(313,314)$. We also observed that Maori and Pacific Islanders had higher rates of diabetes compared to Europeans, which has also been reported in previous studies (315) and may be contributing to the ethnicity disparity in the response to clopidogrel. Our results noting Maori and Pacific Island diabetics having the highest levels of absolute platelet reactivity and HOTPR rates suggests that both ethnicity and diabetes are important contributors to platelet reactivity. However, with the small subgroup sizes we were underpowered to determine whether these are independent predictors, although this trend highlights an interesting question to pursue further.

Treatment with a high dose clopidogrel regimen resulted in greater platelet inhibition and a reduction in the number of patients with HOTPR when compared to a standard (low) dose regiment in our study. However, the absolute reduction in platelet inhibition achieved with high dose clopidogrel was modest and a considerable portion of patients still had HOTPR despite use of a high dose. The ARMYDA-150 mg and 
GRAVITAS randomized controlled trials compared the effect of high and low maintenance dose clopidogrel on platelet inhibition. Consistent with our findings both of these studies demonstrated greater platelet inhibition with high dose clopidogrel $(316,317)$. However, use of high dose clopidogrel has had little impact on clinical events. In the GRAVITAS study treatment with high dose clopidogrel did not reduce adverse events following PCI. The large CURRENT-OASIS 7 study found that short-term treatment with a high dose clopidogrel regimen did not result in a reduction in the primary end point (cardiovascular death or nonfatal $\mathrm{MI}$ ) but did find a significant reduction in stent thrombosis in those undergoing $\mathrm{PCI}$ (318).

This study is also the first to report the effect of prasugrel on platelet function in ACS patients treated with aspirin and clopidogrel who have HOTPR. We found that prasugrel resulted in a consistent and marked reduction in platelet reactivity in this group with an average reduction in residual platelet reactivity of $47 \mathrm{AU}$. Following prasugrel loading, residual platelet reactivity was lowered below the HOTPR cut-point in $89.7 \%$ of patients. Of note, in $34.5 \%$ of patient's residual platelet reactivity was inhibited below the level associated with an increased risk of bleeding. The fact that the majority of HOTPR can be overcome by prasugrel suggests that the variability in the response to clopidogrel is largely due to variability in active metabolite generation. The additional inhibition achieved with prasugrel was of a much greater magnitude than that associated with high dose clopidogrel. However, we can not rule out the potential for a natural decline in platelet reactivity over time independent of prasugrel loading as testing was not repeated on patients that did not receive prasugrel. Although the results of high dose clopidogrel to date have been disappointing, the use of prasugrel as an alternative agent in patients with HOTPR looks promising and the clinical safety and efficacy of this approach is currently being evaluated in clinical trials.

\subsection{Limitations}

As the study was observational, the dose of clopidogrel as well as the timing and use of prasugrel was at the operator's discretion rather than systematically determined by a protocol. The testing of platelet reactivity was not at a standard time after 
symptom onset, or after commencement of dual antiplatelet therapy, and differences in the level of platelet reactivity over time cannot be excluded. Samples were not collected for genetic testing and therefore the role of genetic polymorphism in the phenotypic differences observed could not be assessed. Due to the size of the cohort and the relatively low proportion of Maori and Pacific Islanders, multivariate analysis could not be carried out to determine whether ethnicity, diabetes and clopidogrel dose were independent predictors of HOTPR. Finally, this study was not powered to look at the effect of HOTPR or prasugrel therapy on clinical events.

\subsection{Conclusion}

This study demonstrates that HOTPR is common in a NZ population and occurs more frequently in Maori and Pacific Islanders. The use of high dose clopidogrel resulted in a modest reduction in residual platelet reactivity but a suboptimal response frequently remained. However, use of prasugrel in those with HOTPR following clopidogrel therapy resulted in a consistent and marked reduction in platelet reactivity, which was lowered below the threshold associated with ischaemic risk in the vast majority. This study highlights further questions that exist surrounding the HOTPR phenotype in a NZ ACS population and abroad. Firstly, which predictors of HOTPR are independent risk factors and to what extent is HOTPR in Maori and Pacific Islanders driven by diabetes and CYP2C19 loss of function carriage. Furthermore, is the association between HOTPR and poor outcomes in ACS patients independent of diabetes and ethnicity or is it simply a marker of risk. Our findings suggest that HOTPR can be overcome with more potent P2Y12 inhibitors, but before interventional studies are conducted guided by platelet function testing, a better understanding of this complex and multifactorial phenotype is essential for success. 
CHAPTER 3 
4 Chapter 4: Platelet reactivity genotype and phenotype 


\subsection{Introduction}

The previous chapter identified several clinical predictors of high residual platelet reactivity including ethnicity, diabetes and clopidogrel dose in a NZ ACS population. However, we were not able to investigate the impact of the previously described CYP2C19 genetic variants on clopidogrel response. The prevalence of the CYP2C19*2 allele, resulting in a reduced clopidogrel metabolism, has been reported to be more common in Maori and Pacific Islanders compared to Europeans (230), although the prevalence of this polymorphism is unknown in an NZ ACS population. Furthermore, the prevalence of the CYP2C19*17 allele, associated with significantly lower levels of ADP-induced platelet reactivity (237), is unknown in a NZ ACS population. It is also unknown how these genetic variants impact on residual platelet reactivity and to what extent in the presence of other influencing factors such as diabetes and clopidogrel dose.

In this chapter the overall aim was to further investigate the clinical predictors of high residual platelet reactivity as well as the prevalence and impact of genetic variants in the CYP2C19 gene on platelet reactivity. In addition, multivariate analysis was conducted to determine whether these predictors were independent of each other and determine the most significant drivers of high residual platelet reactivity.

The specific aims of the study were:

1. To determine the clinical, demographic and laboratory parameters that are correlated with high residual platelet reactivity.

2. To identify the prevalence and impact of the CYP2C19* 2 and * 17 alleles on residual platelet reactivity.

3. To investigate the relationship between predictors of residual platelet reactivity using multivariate analysis. 


\subsection{Methods}

\subsubsection{Study Population}

Patients presenting to Wellington Regional Hospital with ACS between January and December 2012 were eligible for inclusion in the study if there was an invasive approach (coronary angiography $\pm \mathrm{PCl}$ ) planned and they were adequately pre-treated with aspirin and clopidogrel. To ensure the necessary statistical power to assess the impact of ethnicity we recruited 282 ACS patients (232 Caucasian, 40 Maori and Pacific Islanders) and enriched the population with an additional 30 Maori and Pacific Islander ACS patients. By enriching the population to have double the Maori and Pacific Islander patients compared to the cohort in Chapter 3 we increased our power to detect a difference in the rate of HOTPR between ethnicities of $15 \%$ or more. An ACS was defined as symptoms suggestive of myocardial ischaemia lasting > 10 minutes and either troponin elevation or $\geq 1 \mathrm{~mm}$ of new ST segment deviation or T wave inversion on an electrocardiogram in at least 2 contiguous leads (289). Adequate pretreatment was defined as chronic therapy with aspirin $(\geq 75 \mathrm{mg})$ and clopidogrel ( $\geq 75 \mathrm{mg}$ ) and / or loading with aspirin $\geq 300 \mathrm{mg}$ at least 2 hours and clopidogrel $\geq 300$ $\mathrm{mg}$ at least 6 hours prior to enrolment. Exclusion criteria included a platelet count less than $100 \times 10^{9} / \mathrm{L}$, known platelet function disorder, administration of a fibrinolytic agent within 24 hours of enrolment or administration of an oral antiplatelet agent other than aspirin of clopidogrel within 2 weeks enrolment. The study was reviewed and approved by the Lower Regional South Ethics Committee (LRS/11/09/035).

\subsubsection{Data Collection}

Patient demographics, clinical characteristics, medications including antiplatelet therapy, clinical management and procedural variables were obtained prospectively from review of the medical records and cardiac catheterization database. Ethnicity was self-identified by the patient. All aspects of clinical management, including prescription of antiplatelet therapy was at the discretion of the attending physicians. 


\subsubsection{Blood Collection and Platelet function testing}

Blood for platelet function testing was collected for testing as described in section 2.2.2. High sensitivity Troponin $\mathrm{T}$ (hs- $\mathrm{TnT}$ ) and electrocardiograms were performed as part of clinical practice by cardiac care nurses prior to cardiac catheterization and following $\mathrm{PCl}$.

\subsubsection{Isolation of Genomic DNA}

Genomic DNA was extracted from EDTA anticoagulated whole blood using the Promega Wizard Genomic DNA Purification Kit (Promega, WI, USA). Following the manufacturer's instructions, $3 \mathrm{~mL}$ of whole blood was added to $9 \mathrm{~mL}$ of red cell lysis buffer and incubated with rocking for 8 minutes at RT. After incubation, cell suspension was centrifuged at $5000 \mathrm{rpm}$ for 5 minutes. Supernatant was discarded leaving a white cell pellet, which was washed in excess sodium chloride $0.9 \%$. The washed white cell pellet was resuspended in nuclei lysis solution $(3 \mathrm{~mL})$ before the addition of protein precipitation solution $(1 \mathrm{ml})$ and vortexed for 30 seconds. Samples were centrifuged at $5000 \mathrm{rpm}$ for 5 minutes and the clear supernatant was transferred in to a separate tube, avoiding the transfer of cellular debris. $3 \mathrm{~mL}$ of isopropanol was added to supernatant and mixed until DNA precipitated out of solution, evident by white threads. Samples were centrifuged for 1 minute at $5000 \mathrm{rpm}$ and the supernatant was decanted to leave a DNA pellet. The DNA pellet was carefully washed with $3 \mathrm{~mL}$ of $70 \%$ ethanol and the remaining pellet was dried in the $37^{\circ} \mathrm{C}$ incubator for 1 hour or until the pellet was clear. Following drying, the pellet was resuspended in $200 \mu \mathrm{L}$ DNA rehydration solution and placed in the $37^{\circ} \mathrm{C}$ incubator overnight to dissolve. DNA suspension was then stored at $4^{\circ} \mathrm{C}$ for genetic analysis.

Genotyping of CYP2C19*2 and *17 was performed by Eng Wee Chau and Leon Smyth under the supervision of Prof. Martin Kennedy from the University of Otago, Christchurch. All DNA samples were normalized to 20-50 ng/ $\mu \mathrm{L}$. However, even after normalisation DNA concentrations were found to be quite variable. Subsequent PCR analysis was performed with greater caution and, as will be described in the following section, ambiguous genotype calls were resolved by various other methods. 


\subsubsection{Validation of CYP2C19*2 and CYP2C19*17 HRM Assays}

All genetic work Both CYP2C19*2 and CYP2C19*17 were genotyped by high-resolution melting (HRM) assays. The genotyping assay for CYP2C19*2 was mainly based on a method previously published by Temesvári et al. (2011) (319), and was further validated in-house. CYP2C19*17 HRM assay was developed and validated on 27 samples whose $-806 \mathrm{C}>\mathrm{T}$ genotypes were determined via a sequencing method described elsewhere in this thesis.

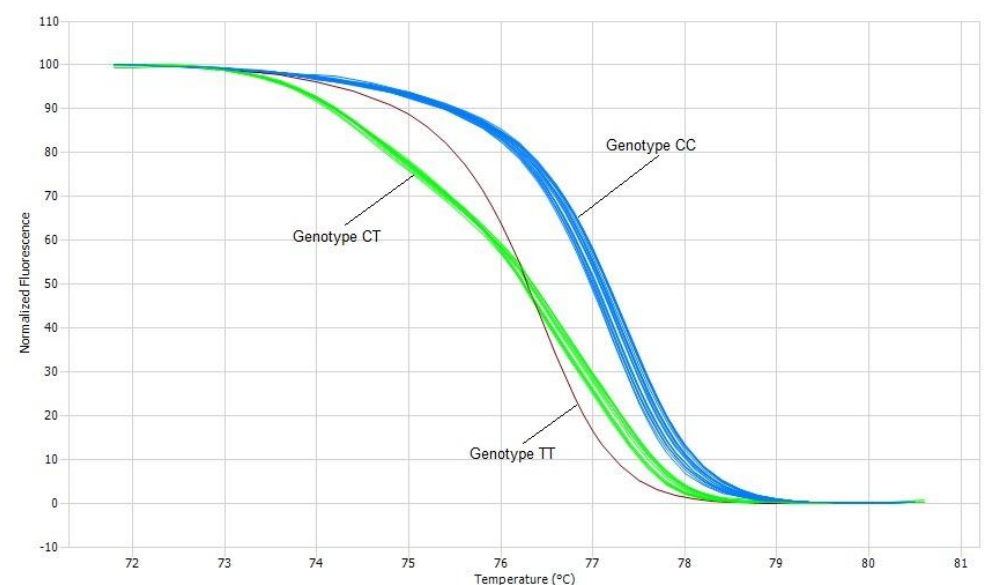

Figure 4-1 High resolution melting curve assay

The defining variant for CYP2C19*17, $-806 \mathrm{C}>\mathrm{T}$ located in the promoter region, was genotyped by a high-resolution melting assay, where three groups of genotypes were defined by clearly distinctive melting profiles: CC (blue), CT (green), and TT (red-brown).

\subsubsection{Genotyping of CYP2C19*2 and CYP2C19*17}

Generally, $10 \mu \mathrm{L}$ PCR reactions were set up and cycled in the Eco Real-Time PCR System (Illumina Inc., California, USA). A no-template control was included in every genotyping run. For CYP2C19*2, a 100-bp fragment was amplified in the following reaction mix: 1X Reaction Buffer $B 1,1.5 \mathrm{mM} \mathrm{Mg}^{2+}, 0.2 \mathrm{mM}$ of each dNTP, $0.2 \mu \mathrm{M}$ of 2C19*2 HRM-F, $0.2 \mu \mathrm{M}$ of 2C19*2 HRM-R, $1.5 \mathrm{mM}$ of SYTO ${ }^{\circledR} 9$ Green Fluorescent Nucleic Acid Stain (Life Technologies, California, USA), and $0.5 \mathrm{U}$ of HOT FIREPol ${ }^{\circledR}$ DNA Polymerase (Solis BioDyne, Estonia). The thermal profile consisted of an initial activation step of $95{ }^{\circ} \mathrm{C}$ for $10 \mathrm{~min}$, then 40 cycles of $95^{\circ} \mathrm{C}$ for 10 seconds (s), $54{ }^{\circ} \mathrm{C}$ for $30 \mathrm{~s}$, and $72{ }^{\circ} \mathrm{C}$ for $30 \mathrm{~s}$. For CYP2C19*17, a 62-bp fragment was amplified with $1 \mathrm{X}$ Reaction Buffer B1, 1.5 mM Mg${ }^{2+}, 0.2 \mathrm{mM}$ of each dNTP, $0.2 \mu \mathrm{M}$ of 2C19*17 HRM LitF, $0.2 \mu \mathrm{M}$ of $2 \mathrm{C} 19 * 17$ HRM LongR, $1.5 \mathrm{mM}^{*}$ SYTO $^{\circledR} 9$ Green Fluorescent Nucleic Acid Stain 
(Life Technologies, California, United States), and $0.5 \mathrm{U}$ of HOT FIREPol ${ }^{\circledR}$ DNA Polymerase (Solis BioDyne, Estonia). The cycling conditions were $95{ }^{\circ} \mathrm{C}$ for $10 \mathrm{~min}$, then 45 cycles of $95{ }^{\circ} \mathrm{C}$ for $10 \mathrm{~s}, 52{ }^{\circ} \mathrm{C}$ for $15 \mathrm{~s}$, and $72{ }^{\circ} \mathrm{C}$ for $15 \mathrm{~s}$. To generate a HRM profile, all PCR products were completely denatured by heating at $95{ }^{\circ} \mathrm{C}$ for $15 \mathrm{~s}$, allowed to re-anneal at $55{ }^{\circ} \mathrm{C}$ for $15 \mathrm{~s}$, and denatured again by gradual heating to 95 ${ }^{\circ} \mathrm{C}$, during which fluorescence data was collected every $0.1{ }^{\circ} \mathrm{C}$. Ambiguous genotype calls i.e., samples which had an unusual melting curve visually, were resolved by Sanger sequencing or restriction digest.

Table 4-1 Primer Sequences

\begin{tabular}{|l|l|}
\hline Primer name & Primer sequence (5'-3') \\
\hline 2C19*17 HRM-F & CTTAGATATGCAATAATTTTCCCAC \\
\hline 2C19*17 HRM-R & CTTTCCATAAAAGCAAGGTT \\
\hline 2C19*17 HRM LitF & AAATTTGTGTCTTCTGTTCTCAAA \\
\hline 2C19*17 HRM LongR & TGCCCATCGTGGCGCATTAT \\
\hline 2C19Ex5F & CAACCAGAGCTTGGCATATTG \\
\hline 2C19Ex5R & TGATGCTTACTGGATATTCATGC \\
\hline 2C19Prom4F & GCCCTTAGCACCAAATTCTCT \\
\hline 2C19Prom4R & CACCTTTACCATTTAACCCCC \\
\hline
\end{tabular}

\subsubsection{Restriction Fragment Length Polymorphism (RFLP) and Sanger Sequencing}

For RFLP analysis of CYP2C19*2, a 409-bp fragment was amplified in a $10 \mu \mathrm{L}$ reaction containing 1X Reaction Buffer, $1.5 \mathrm{mM} \mathrm{Mg}{ }^{2+}, 0.2 \mathrm{mM}$ of each dNTP, $0.2 \mu \mathrm{M}$ of 2C19Ex5F, $0.2 \mu \mathrm{M}$ of 2C19Ex5R, and 0.25 U of TAQ-TI Heat-Activated DNA Polymerase (Fisher Biotec, Western Australia). The thermal profile consisted of an initial denaturation step at $94{ }^{\circ} \mathrm{C}$ for $2 \mathrm{~min}$, followed by 35 cycles of $94{ }^{\circ} \mathrm{C}$ for $15 \mathrm{~s}, 56^{\circ} \mathrm{C}$ for $15 \mathrm{~s}$, and $72{ }^{\circ} \mathrm{C}$ for $30 \mathrm{~s}$, and a final elongation step of $72{ }^{\circ} \mathrm{C}$ for $1 \mathrm{~min}$. Restriction digest was carried out by directly incubating the PCR mix with $10 \mathrm{U}$ of Smal (New England BioLabs Inc., Massachusetts, USA) at room temperature for two hours. Digested PCR products were resolved by $1.5 \%$ agarose gel electrophoresis. The presence of CYP2C19*2 abolishes Smal recognition site; as a result the PCR fragment 
would remain undigested. A 483-bp fragment encompassing the $-806 \mathrm{C}>\mathrm{T}$ variant was similarly amplified with the primer pair 2C19Prom4F and 2C19Prom4R; the reaction volume was increased to $20 \mu \mathrm{L}$ and annealing temperature to $65^{\circ} \mathrm{C}$. The resulting PCR products were purified through the AcroPrep ${ }^{\mathrm{TM}} 384$ Filter Plate, Omega 30K (Pall Corporation, Michigan, USA), and subsequently Sanger-sequenced using the BigDye ${ }^{\circledR}$ Terminator v3.1 Cycle Sequencing Kit as per the manufacturer's instructions.

\subsubsection{Definitions}

The clopidogrel dose was defined as "high" if patients had received a $600 \mathrm{mg}$ loading dose followed by a $150 \mathrm{mg}$ daily maintenance dosing. Intermediate dose was defined as either a $600 \mathrm{mg}$ loading dose followed by $75 \mathrm{mg}$ daily maintenance dose or a $300 \mathrm{mg}$ loading dose coupled with 150 mg maintenance dose. Low dose was defined as a 300 mg loading dose followed by $75 \mathrm{mg}$ daily maintenance dose or chronic therapy with 75 mg daily of clopidogrel. Patients with a platelet count over $300 \times 10^{9} / \mathrm{L}$ were defined as having a high platelet count. Obesity was defined as a BMI $\geq 30$ and the classification of loss of function (LOF) allele and gain of function (GOF) allele was either heterozygous or homozygous for CYP2C19*2 SNP and CYP2C19*17 respectively.

\subsubsection{Statistical analysis}

In the previous study, the continuum of residual platelet reactivity was categorised in a binary fashion into HOTPR and non-HOTPR using the cut-point of $47 \mathrm{AU}$ as previously described by the Sibbing group (184). This method was used to allow us to identity the prevalence of HOTPR in a NZ population and compare the rate with other reported studies using the same methods. The limitation with using a defined cut point is you lose information and miss potentially important relationships. In this study we wanted to further investigate the relationship between clinical and genetic factors with high and low residual platelet reactivity. To have the most sensitivity we decided to analyse the relationship between these factors with residual platelet reactivity as a continuous variable. 
Continuous variables are expressed as median and interquartile range (IQR). Categorical variables are expressed as frequencies and percentages. We compared the proportion of Maori and Pacific Islanders or Europeans with diabetes, LOF and GOF alleles using the Chi-Squared test. Platelet reactivity distribution did not pass the D'Agostino \& Pearson omnibus normality test. Therefore all univariate analysis was carried out using non-parametric tests. Absolute values for residual platelet reactivity (AU) were compared by ethnicity, diabetes, dose groups, high platelet count, obesity and LOF and GOF genotypes using the Mann-Whitney test. The relationship between age, renal function and residual platelet reactivity was assessed by Spearman's correlation coefficient ( $r h o)$. To perform parametric multivariate analysis, platelet reactivity was log transformed and compared against significant univariate predictors of residual platelet reactivity using a multiple linear regression model. Regression analysis and Chi-Squared tests were performed using PASW 18.0 (IBM, NY, USA), all other analysis and graphs were generated using GraphPad prism 5 (GraphPad Software Inc., CA, USA). 


\subsection{Results}

\subsubsection{Demographics}

During the study period 312 patients were enrolled in the study. The baseline demographics for the population are displayed in Table 4-2. This study cohort had a higher proportion of Maori and Pacific Islanders (22.4\%) with the additional selected recruitment of these patients compared to the study population in Chapter 3, whereas all other demographics were comparable.

Table 4-2 Patient baseline demographics

\begin{tabular}{|l|l|}
\hline & $\mathrm{N}=312$ \\
\hline Age (years) & $63(31-89)$ \\
\hline Male, $\mathrm{n}(\%)$ & $225(72)$ \\
\hline BMI & $28(17-50)$ \\
\hline Ethnicity, $\mathrm{n}(\%)$ & \\
\hline European & $232(74.3)$ \\
\hline Maori and Pacific Islander & $70(22.4)$ \\
\hline Other & $10(3.2)$ \\
\hline Risk Factors, $\mathrm{n}(\%)$ & \\
\hline Hypertension & $213(68)$ \\
\hline Dyslipidaemia & $209(66.9)$ \\
\hline Diabetes & $79(25.3)$ \\
\hline Current Smoker & $60(19.2)$ \\
\hline Medical History, $\mathrm{n}(\%)$ & \\
\hline Previous MI & $93(29.8)$ \\
\hline Previous PCl & $52(16.6)$ \\
\hline Previous CABG & $21(6.7)$ \\
\hline Clinical Presentation, $\mathrm{n}(\%)$ & \\
\hline STEMl & $58(18.5)$ \\
\hline NSTEMI & $239(76.6)$ \\
\hline Unstable Angina & $15(4.8)$ \\
\hline Laboratory data & \\
\hline Creatinine (umol/L) & $84(51-1058)$ \\
\hline Platelet Count (10\%) $/ \mathrm{L})$ & $220(109-626)$ \\
\hline
\end{tabular}




\subsubsection{Predictors of platelet reactivity}

To investigate the impact of different predictors on platelet reactivity, demographics, clinical characteristics and laboratory data were tested against absolute levels of platelet reactivity in univariate analysis. Patients with diabetes had significantly higher levels of platelet reactivity compared to non-diabetics (44 AU (4-140) vs. 34 AU (4148), $p=0.002$, Figure 4-2A. Platelet reactivity was also compared between patients that received different doses of clopidogrel. As with the last cohort there was significant variance in the dose of clopidogrel that patients were allocated, with $50 \%$ of patients receiving low dose clopidogrel, $22 \%$ on a medium dose and $28 \%$ on a high dose. Patients receiving low doses of clopidogrel had significantly higher levels of platelet reactivity compared to the medium or high dose patients (43 AU (6-143) vs. $31 \mathrm{AU}(6-140), \mathrm{p}=0.013$, and $31 \mathrm{AU}(4-104), \mathrm{p}=0.002$, Figure 4-2B). There was no difference in platelet reactivity between medium and high dose patient groups.

Platelet count was measured in all patients, and patients with a high platelet count had a level of platelet reactivity 1.8 times higher than patients with a normal platelet count (53 AU $(6-148)$ vs. $34 \mathrm{AU}(4-104), p=0.001$, Figure $4-2 C)$. Similarly, patients who were obese as defined by a BMI $\geq 30$, had significantly higher levels of platelet reactivity compared to patients with a $\mathrm{BMI}<30(40 \mathrm{AU}(4-148)$ vs. $33(4-141), \mathrm{p}=$ 0.01 , Figure 4-2D) 

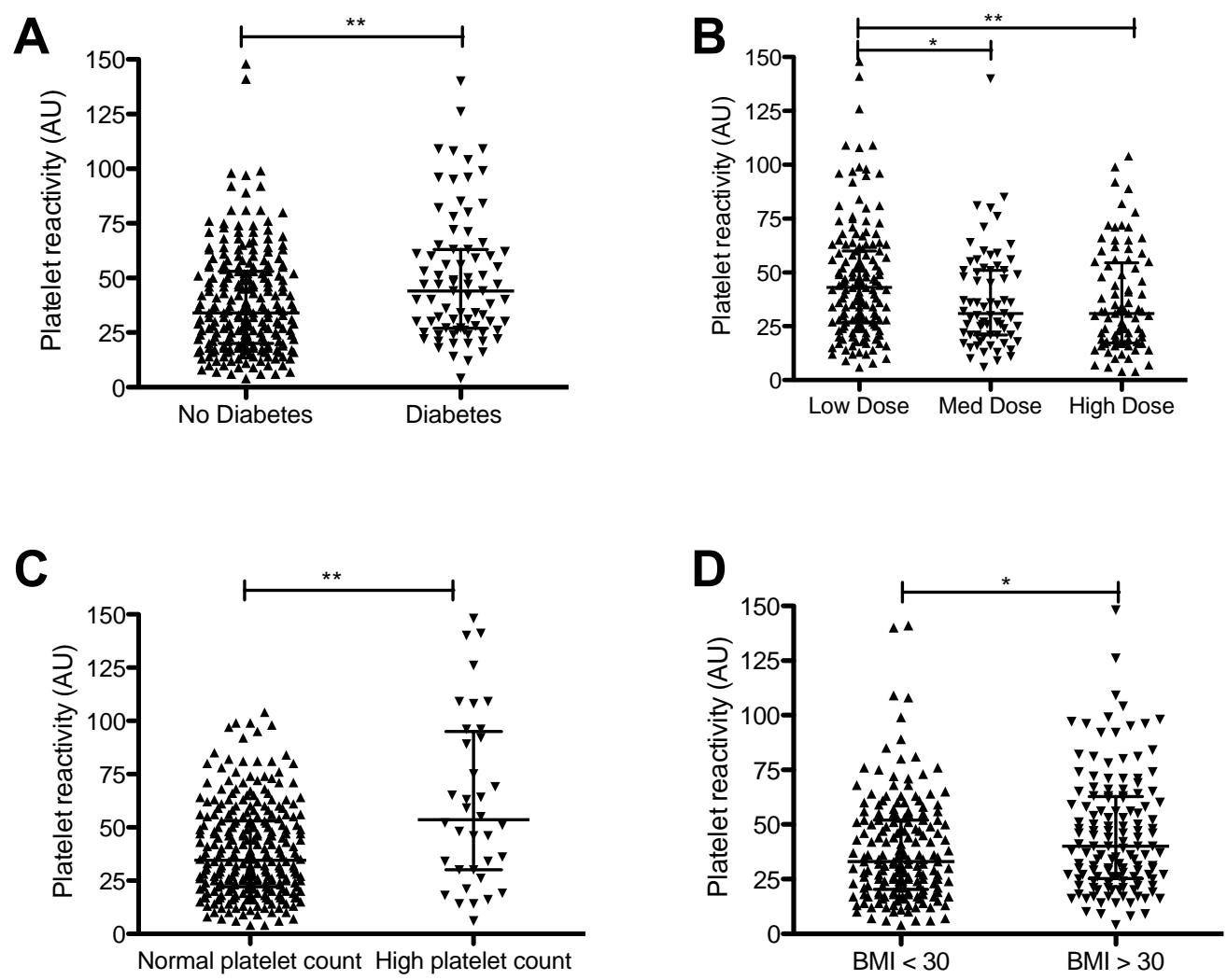

Figure 4-2 Clinical predictors of platelet reactivity

Absolute levels of platelet reactivity (AU) as measured by the Multiplate in patients with diabetes and no diabetes (A), by clopidogrel dose group including low, medium and high doses (B). Platelet reactivity was also compared by high platelet count $\left(>300 \times 10^{9} / \mathrm{L}\right)$ vs. normal platelet count $\left(<300 \times 10^{9} / \mathrm{L}\right)(\mathrm{C})$ and by BMI (D). Data presented as Median and IQR, analysed using the Mann-Whitney test. ${ }^{*} p<0.05,{ }^{* *} p<0.01$. 
The impact of the SNPs CYP2C19*2 and CYP2C19*17 on platelet reactivity was also investigated. The prevalence of CYP2C19*2 was high in the cohort with $32 \%$ (100) of patients having at least 1 of the loss of function (LOF) allele, 28.5\% (89) were heterozygous for the LOF allele and 3.5\% (11) were homozygous for the LOF allele. The carriage of at least one LOF allele (Figure 4-3A) resulted in 1.3 times higher levels of platelet reactivity compared to wild type (w/t) (44 AU $(6-126)$ vs. $33(4-148), p=$ 0.02). However, when the different genotypes were separated (Figure 4-3B), the highest levels of platelet reactivity were seen in LOF homozygotes $\left(*^{*} 2 /{ }^{*} 2\right)$ and were significantly higher compared to w/t (59 AU (20-126) vs. $33 \mathrm{AU}(4-148), \mathrm{p} 0.03)$. The heterozygotes had a numerically higher level of platelet reactivity (43 AU (6 - 109), but this was not significantly different to $w / t$ (33 Au (4-148), $p=0.07$ ) or homozygotes (59 AU $(20-126), p=0.2)$.
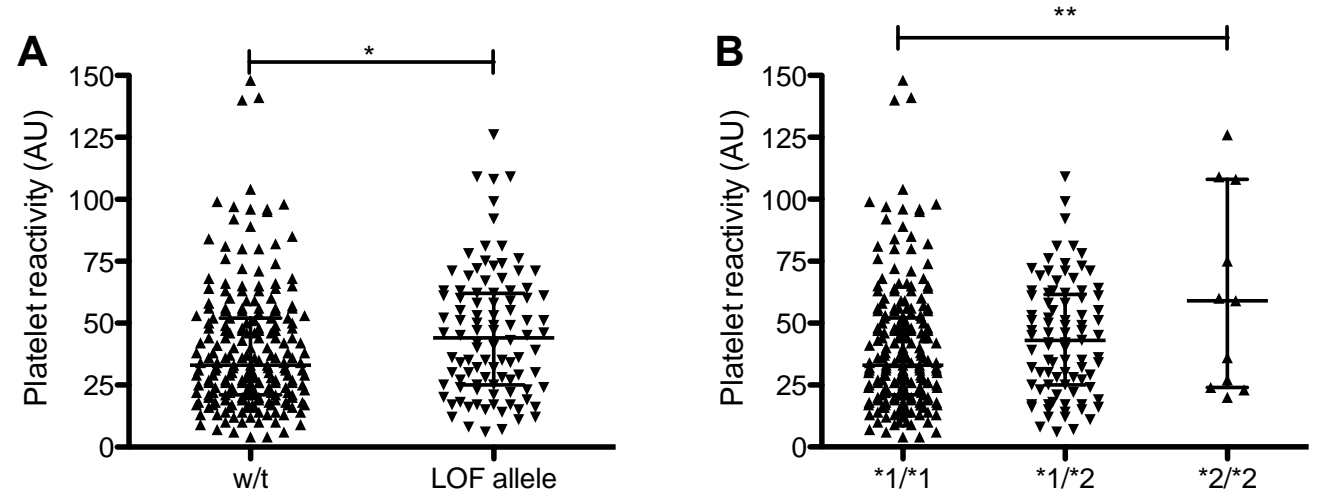

Figure 4-3 CYP2C19*2 loss of function allele (LOF) and platelet reactivity

Absolute level of platelet reactivity $(A U)$ as measured by Multiplate, in patients wild type (w/t) and at least 1 loss of function (LOF) allele (A). Platelet reactivity was also compared between the different CYP2C $19^{*} 2$ genotypes, $1^{*} / 1^{*}$ - wild type, $1^{*} / 2^{*}$ heterozygote and ${ }^{*} 2{ }^{*} 2$ homozygote (B). Data presented as Median and IQR, analysed using the Mann-Whitney test. ${ }^{*} p<0.05$, ${ }^{* *} p$ $<0.01$. 
The gain of function SNP CYP2C19*17 was also identified in patients and the levels of platelet reactivity were compared between genotypes. The prevalence of CYP2C19*17 was also high in the cohort with 34\% (106) of patients having at least 1 of the gain of function (GOF) allele, 30.4\% (95) were heterozygous for the GOF allele and 3.5\% (11) were homozygous for the GOF allele. The carriage of at least $1 \mathrm{GOF}$ allele did not have any noticeable impact on platelet reactivity levels (Figure 4-4A, 33 $\mathrm{AU}(4-141)$ vs. $36 \mathrm{AU}(4-148), \mathrm{p}=0.3)$. Furthermore, analysis of the different genotypes (Figure 4-4B) also displays little difference between wild type, heterozygous and homozygote genotypes on platelet reactivity (36 AU (4 - 148), 36 $\mathrm{AU}(6-140), 31(4-141), p=0.47)$.
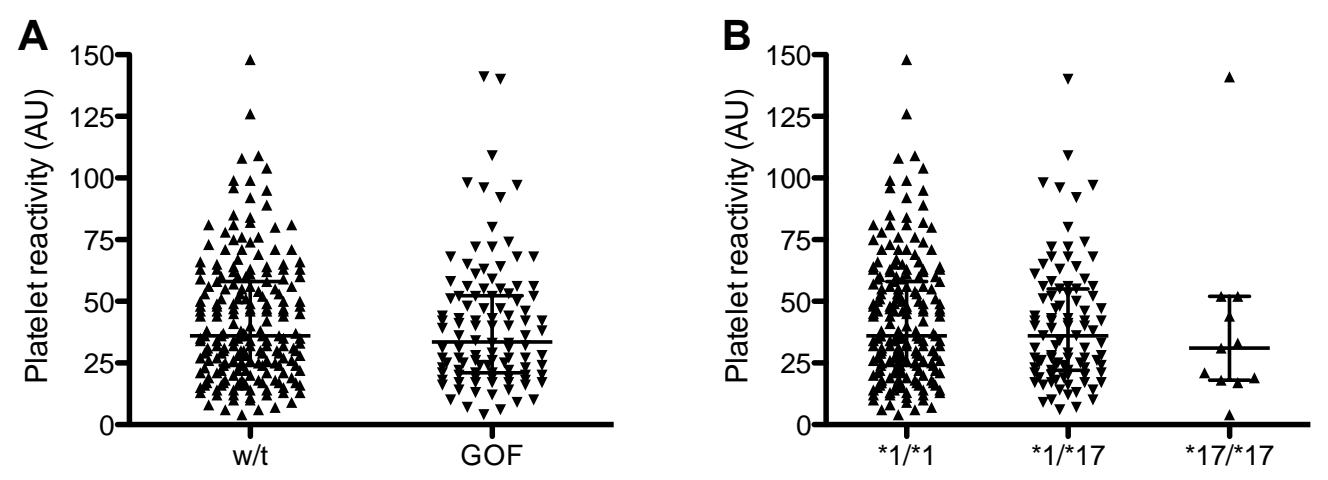

Figure 4-4 CYP2C19*17 Gain of Function (GOF) allele and platelet reactivity

Absolute level of platelet reactivity ( $A U)$ as measured by the Multiplate, in patients wild type $(\mathrm{w} / \mathrm{t})$ and at least 1 gain of function (GOF) allele (A). Platelet reactivity was also compared by CYP2C $19^{*} 17$ genotype, $1^{*} / 1^{*}$ - wild type, $1^{*} / 17^{*}$ heterozygote and ${ }^{*} 17 /^{*} 17$ homozygote (B) Data presented as Median and IQR, analysed using the Mann-Whitney test

The level of platelet reactivity was not significantly different between ethnicities (Figure 4-5A), although numerically Maori and Pacific Islanders had slightly higher levels of platelet reactivity compared to Europeans (38 AU (4 - 145) vs. $34 \mathrm{AU}(4-$ 141), $p=0.14)$. Of note, Maori and Pacific Islanders had both higher rates of diabetes (35.7\% vs. $21.5 \%, \mathrm{p}=0.032$, Figure $4-5 \mathrm{~B})$ and a higher prevalence of the CYP2C19*2 LOF allele ( $47 \%$ vs. $26 \%, p=0.001$, Figure $4-5 C$ ) compared to Europeans. Interestingly, Maori and Pacific Islanders also had a significantly lower prevalence of the CYP2C19*17 GOF allele ( $11.4 \%$ vs. $41.2 \%, p=0.0001$, Figure $4-5 D)$ compared to Europeans. 

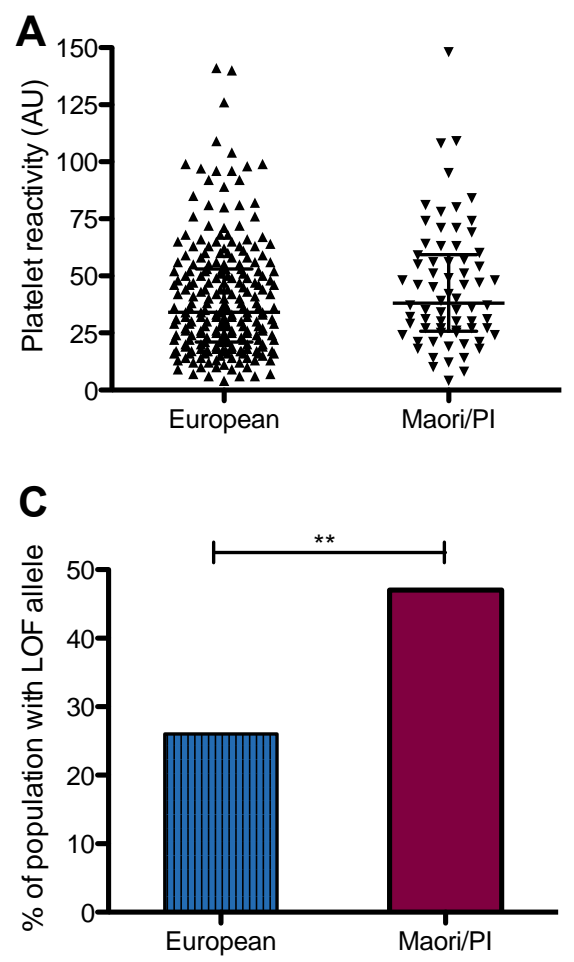

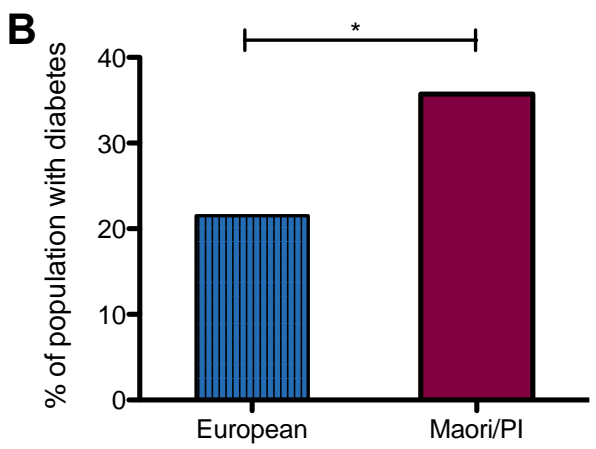

D

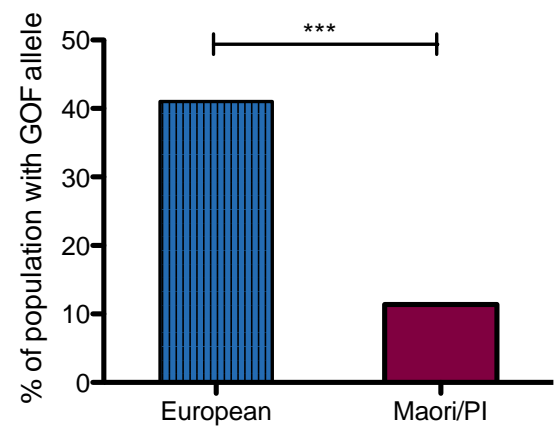

Figure 4-5 Ethnicity and platelet reactivity

Absolute levels of platelet reactivity (AU) as measured by the Multiplate in European and Maori and Pacific Island patients (A). Data presented as medians and IQR. The rate of patients with diabetes (B) and the proportion of patients positive for the LOF allele (C) and GOF allele (D) in European vs. Maori and Pacific Islander ethnicities, proportion of population (\%) shown for each. Analysis performed by student $t$ test. ${ }^{*} p<0.05,{ }^{* *} p<0.01,{ }^{* * *} p<0.001$.

There was no significant correlation between age ( $r$ ho $=0.002, p=0.2$ ) or renal function ( $r h o=0.0001, p=0.8$ ) as measured by creatinine level and residual platelet reactivity. Mean residual platelet reactivity was also not affected by concomitant treatment with a proton pump inhibitor or the type of ACS at presentation. There were no clinical, demographic or laboratory variables such as renal function, age and sex, that significantly correlated with low levels of platelet reactivity in this cohort. 


\subsubsection{Multivariate analysis of platelet reactivity predictors}

To test whether predictors that were found to be significant indicators of high levels of platelet reactivity in univariate analysis were independent predictors, multivariate linear regression analysis was performed on the following variables: obesity, diabetes, high platelet count, LOF allele carriage and clopidogrel low dose (Table 4-3). The model suggested these variables accounted for $11 \%$ (adjusted $R^{2} 0.110$ ) of the variance in platelet reactivity. Of these variables, diabetes, high platelet count and low clopidogrel dose were found to be independent predictors of high platelet reactivity. Obesity and LOF allele carriage did not independently predict platelet reactivity. High platelet count had the greatest impact on platelet reactivity with a $\beta$ coefficient of $0.151 \pm 0.05(p=0.002)$, followed by diabetes $0.094 \pm 0.03(p=0.009)$ and clopidogrel low dose $0.084 \pm 0.03(p=0.006)$.

Table 4-3 Multivariate Linear Regression Coefficients

\begin{tabular}{|l|l|l|l|l|}
\hline Variable & Coefficient $(\beta)$ & Std. Error & Standardized $\beta$ & $p$ Value \\
\hline Intercept & 1.588 & 0.05 & & 0.0001 \\
\hline Obesity & 0.043 & 0.03 & 0.075 & 0.179 \\
\hline Diabetes & 0.094 & 0.03 & 0.145 & 0.009 \\
\hline High platelet count & 0.151 & 0.05 & 0.172 & 0.002 \\
\hline LOF allele carriage & 0.051 & 0.03 & 0.084 & 0.126 \\
\hline Clopidogrel low dose & 0.084 & 0.03 & 0.152 & 0.006 \\
\hline
\end{tabular}




\subsection{Discussion}

This study sought to further investigate the complex nature of residual platelet reactivity in ACS patients and the different clinical, demographic, genetic and laboratory parameters that influence platelet reactivity. Of the different parameters that were collected, diabetes, clopidogrel dose, high platelet count, obesity and carriage of the LOF allele were all correlated with high residual platelet reactivity in univariate analysis. The prevalence of both the LOF allele and GOF allele was high in the NZ ACS population, with particularly high rates of CYP2C19*2 in Maori and Pacific Islanders. In multivariate analysis, only diabetes, high platelet count and clopidogrel dose were independently associated with higher levels of residual platelet reactivity.

As described in the previous chapter, diabetes is a state of increased platelet activity and aggregation and in this cohort; diabetes continues to be a strong predictor of high residual platelet reactivity. The presence of diabetes resulted in a 3-fold increase in platelet reactivity compared to patients without diabetes. In this cohort we were able to perform multivariate analysis and determined that diabetes is an independent and significant risk factor for high platelet reactivity. Other studies have also found absolute platelet reactivity levels to be higher and the rate of non-responders to be more common in patients with diabetes $(310,320,321)$. Previous studies have demonstrated that platelets are hyper-reactive in patients with diabetes and result in a poor response to P2Y12 inhibitors. Proposed mechanisms include: increased glycation of platelet membrane proteins, which may alter P2Y12 functioning; overexpression of platelet integrins, such as glycoprotein Ilb/IIla; disturbed calcium homeostasis and enhanced inflammatory status $(322,323)$. Some studies propose that high platelet reactivity can be attributed to a higher BMI in diabetic patients (326). However our data demonstrates diabetes as a risk factor independent of BMI.

Furthermore, as observed in the previous cohort, clopidogrel dose was a significant independent predictor of high platelet reactivity and the administration of low doses correlated with significant increases in platelet reactivity. The administration of medium or high doses of clopidogrel resulted in a modest decrease in platelet 
reactivity compared to low doses. Although there was no difference between medium and high doses suggesting a threshold of platelet inhibition that clopidogrel can achieve.

The factor that had the greatest impact on high residual platelet reactivity in the multivariate model was a high platelet count. This was not identified as a significant contributor to platelet reactivity in the previous cohort but high platelet count has been reported in earlier studies to correlate with high residual platelet reactivity (236). There may be several reasons as to why higher platelet counts are contributing to higher levels of platelet reactivity. It is reasonable to argue that increases in platelet numbers and therefore an increase in P2Y12 receptor targets, would lead to higher rates of residual platelet reactivity due to the consequential insufficient dose of clopidogrel. Other studies have also hypothesized that an increase in platelet turnover can contribute to higher levels of residual platelet reactivity $(325,326)$. A high platelet turnover is often observed in ACS when platelets are being consumed by thrombus and is associated with a high platelet count, which results in an increase in a population of immature reticulated platelets (329). Reticulated platelets represent younger, more hyperactive platelets, with an increased volume and greater number of dense granules than older circulating platelets (328). They also have a greater prothrombotic potential; they aggregate more rapidly with collagen and have higher levels of intracellular thromboxane A2 as well as expression of P-selectin and Gpllb/IIla $(329,330)$. These factors combined may reduce the ability of antiplatelet therapies to effectively inhibit aggregation and result in higher levels of residual platelet reactivity. Lastly, it has been found that low platelet count can influence Multiplate testing independent of platelet function, but this has not been the case for high platelet count (331) (332). Further investigation is required to determine if high platelet count is a risk factor for high residual platelet reactivity, independent of the platelet function assay being used.

One of the central findings in this study is the high prevalence of the CYP2C19*2 LOF allele displayed in a NZ ACS population and the significant difference in expression between ethnicities. The presence of at least 1 LOF allele was also a significant 
univariate predictor of high residual platelet reactivity, which was largely driven by the high platelet reactivity levels found in patients homozygous for the CYP2C19*2 polymorphism. Studies conducted in the general NZ population have shown a higher prevalence of $* 2$ alleles at $24 \%$ in Maori and Pacific Islanders compared to NZ Europeans at $13 \%(229,333)$. However, our study in ACS patients found a much higher prevalence of the CYP2C19*2 LOF compared to previous findings, with $32 \%$ of the overall population and $47 \%$ of Maori and Pacific Islanders positive for the allele. Other studies have found significant differences in the reported rate of CYP2C19 polymorphisms in the same race (227). For example, in two studies of Caucasian ethnicity, CYP2C19*3 frequency was reported at 0 and $6.5 \%(334,335)$ and in two studies of African American ethnicity, CYP2C19*2 frequency was reported at 18 and $25 \%(336)$. These differences are most likely due to the effect of low sample numbers on reported rates. This may also explain the discrepancy between our reported rate of LOF allele carriage in Maori and Pacific Islanders and that reported by Lea et al. (230) who also had a small cohort size of 60 Maori and Pacific Islanders. This is not the first study to find differences in the rate of CYP2C19 polymorphisms between ethnicities. However, in comparison Maori and Pacific Islanders have a high prevalence of the CYP2C19*2 LOF allele. A cumulative meta-analysis comparing the frequency of the CYP2C19 polymorphisms found African American, American and Europeans have the lowest average prevalence of CYP2C19*2 at 15\%, followed by East Asian at $29 \%$ and South/central Asian at 35\% (227).

Reduced clopidogrel response in CYP2C19*2 carriers has been established from pharmocokinetic and pharmodynamic studies, which lead to the FDA black box warning to prescribe clopidogrel based on CYP2C19 genotype (337). The impact of CYP2C19*2 carriage on MACE risk is debatable in the literature with some metaanalyses reporting an increase in cardiovascular risk, particularly stent thrombosis in patients undergoing percutaneous coronary intervention (PCI) $(232,233)$. However other studies have directly challenged this link and report a marginal increase in MACE for patients with the LOF allele $(234,235)$. The reported inconsistencies linking CYP2C19*2 and MACE are most likely due to the small influence (5-12\%) the LOF allele has on the variability in clopidogrel response (236). In addition, our study found the 
LOF allele was not an independent predictor of high residual platelet reactivity and is not significantly contributing to the variability seen in platelet reactivity in this ACS cohort. As discussed in section 1.4.11, genetic polymorphisms also affect other genes that are central to active clopidogrel metabolite production and a combination of these polymorphisms may have significant influence on residual platelet reactivity beyond CYP2C19*2.

The gain of function (GOF) CYP2C19*17 polymorphism has been associated with significantly lower levels of ADP-induced platelet aggregation or Low on treatment platelet reactivity (LOWPR) in previous studies (237). In contrast, our study demonstrated no meaningful change in platelet reactivity between patients who were wild type, heterozygotes or homozygotes for the *17 polymorphism. However, we did find a significant difference in GOF allele prevalence between ethnicities. The carriage of the GOF allele has been reported to be more prevalent in Caucasians (21\%) compared to other ethnicities (228) and our study further supports this with an even higher rate at $41 \%$ of Europeans positive for the GOF allele. Our study is also the first to report the prevalence of the CYP2C19*17 polymorphism in Maori and Pacific Islanders which was found to be significantly lower than Europeans at $11 \%$. This rate is similar to what is seen in African American populations (16\%) and higher than reports from Asian populations (4\%) (228).

LOWPR or hyper-response to clopidogrel measured by platelet function assays have been linked to increased major bleeding in both $\mathrm{PCl}$ treated patients and those going on to cardiac surgery (338-340). There has also been enhanced interest in using platelet function testing to guide antiplatelet therapy treatment particularly before CABG surgery as a number of studies have shown LOWPR to be predictive of an increased transfusion requirement post-surgery $(341,342)$. A consensus regarding the impact of the GOF allele on platelet reactivity and clinical outcomes has yet to be reached and our findings would suggest the GOF allele does not impact residual platelet reactivity significantly. Interestingly, we also did not encounter any other clinical or demographic parameter that was significantly correlated with lower levels of residual platelet reactivity. 
In this cohort we did not find a significant difference in residual platelet reactivity between ethnicities. This finding is surprising given our results from the previous chapter, showing a significant increase in HOTPR in Maori and Pacific Islanders. This finding was also one of the key drivers to explore the HOTPR phenotype further and was one of the reasons to selectively recruit a larger proportion of Maori and Pacific Islanders. Furthermore, Maori and Pacific Islanders did have significantly higher rates of diabetes and the LOF allele, which were both correlated with higher levels of platelet reactivity. However, a small trend of increased platelet reactivity was demonstrated in this cohort and this finding in a larger group of participants suggest that ethnic differences are only having a minor influence on platelet reactivity. Maori and Pacific Islanders are a high-risk population with considerably poor cardiovascular outcomes compared to the general population. Further investigation into the factors contributing to cardiovascular risk is essential to tackling the health inequalities seen in these ethnicities.

The finding that only a small percentage of the variability in platelet reactivity can be attributed to diabetes, clopidogrel dose and platelet count, indicates there still remains unidentified factors that are having a significant impact on platelet reactivity and highlights the complexity of this phenotype. One potential factor that will undoubtedly be having a large influence on platelet reactivity is the heightened inflammatory state and abundance of platelet activating factors that are released during an ACS. By measuring platelet function during this time, the background noise of inflammation may be overshadowing the impact of other predictors on residual platelet reactivity, particularly those that remain in a constant state such as genetic polymorphisms. Diabetes and platelet count are known to be dynamic factors that respond to changes in inflammation and oxidative stress and may track with ACS progression and resolution. This may explain why diabetes and platelet count are the most significant predictors of residual platelet reactivity in our ACS cohort.

The optimal time to measure platelet function is at the centre of significant debate and an important issue when considering personalising antiplatelet therapy based on 
platelet function assays. Some studies have suggested that platelet function testing at the time of an ACS is an unreliable predictor of the antiplatelet response during maintenance therapy (343). This finding again highlights the dynamic nature of platelet reactivity and suggests clinical factors that are influencing residual platelet reactivity at the time of infarct, may not be the same factors or impacting to the same degree on platelet reactivity during maintenance therapy. However, it is also important to point out that the key purpose of platelet function testing is to identify patients at increased risk, both ischaemic and bleeding risk. This risk is highest directly after an ACS (343) and potentially the most benefit from altering antiplatelet therapy is in this early period. Therefore, it is important to investigate platelet function as well as other clinical and genetic factors and determine which variables or combination of variables is the most predictive of ischaemic and bleeding outcomes.

\subsection{Limitations}

As the study was observational, the dose and loading time of clopidogrel was at the operator's discretion rather than systematically determined by a protocol. The testing of platelet reactivity was not at a standard time after symptom onset, or after commencement of dual antiplatelet therapy, and differences in the level of platelet reactivity over time cannot be excluded. However, all blood samples were taken before angiography and no longer than 7 days post admission to limit this variance. The inclusion/exclusion criteria were also designed to ensure all patients would experience the maximal effect from antiplatelet therapy at the time of testing.

\subsection{Conclusion}

This study highlights the complex nature of residual platelet reactivity during an ACS, with many components influencing the response to clopidogrel. Diabetes, high platelet count and clopidogrel dose were all significant and independent predictors of high residual platelet reactivity. However, only a small percentage of the variability in platelet reactivity could be attributed to these predictors suggesting unidentified factors are having considerable influence on residual platelet reactivity. The large inter-individual variability in the response to clopidogrel can be considered as a matter of systems pharmacology rather than a phenomenon determined by a single-gene or 
clinical factor, and prediction of an individual's response to clopidogrel may require integration of information on genetics and clinical variables, both captured in an individual's on-treatment platelet reactivity status. Furthermore, determining the ability of this phenotype to predict patient risk, potentially integrated with other patient parameters, is central to progressing platelet function testing as a viable clinical tool. 
${ }_{5}$ Chapter 5: Platelet reactivity and outcomes in Acute Coronary Syndromes 


\subsection{Introduction}

In the preceding chapter we demonstrated that several clinical factors contributed to the observed variability in residual platelet reactivity. In particular, diabetes, high platelet count and a low clopidogrel dose were all significant independent predictors of high residual platelet reactivity while the CYP2C19*2 genotype had only a minor impact on the platelet reactivity phenotype. Previous studies have demonstrated separately that the HOTPR phenotype $(344)$ or LOF genotype $(232,233)$ are associated with an increased risk of major adverse cardiovascular (MACE) events in various CAD populations. However, outcome studies in these populations have not demonstrated whether the increased risk attributed to either phenotype or genotype are independent of each other. Furthermore, it has not been investigated whether the HOTPR phenotype predicts MACE risk independently of important clinical risk factors such as diabetes, which we know contributes to high residual platelet reactivity.

Therefore the aims of this chapter were:

1. To investigate the relationship between phenotype, genotype and predictors of phenotype with MACE and bleeding events in an ACS population and whether these various predictors were independent of one another.

2. In addition to investigating risk in an ACS population, to also explore the relationship between platelet reactivity and risk in the patients undergoing $\mathrm{PCl}$, as this population may benefit the most from personalized antiplatelet therapy.

\subsection{Methods}

\subsubsection{Study population}

From the recruited cohort in Chapter 4, 294 (95\%) of the patients had completed follow up to 1 year. Of the remaining 18 patients, 10 patients had medical documents that could not be accessed and 8 patients were unreachable by phone. 


\subsubsection{Data collection}

Patient demographics, clinical characteristics, medications including antiplatelet therapy, clinical management and procedural variables were obtained prospectively from review of the medical records and cardiac catheterisation database. Cardiology registrars at Wellington Hospital collected procedural variables and in-hospital outcomes. The cardiology research nurses collected follow-up data with telephone calls at 30 days and 1 year. Where necessary, a review of case notes was performed and the appropriate general practitioner contacted to further classify clinical outcomes.

\subsubsection{Platelet function testing and Genotyping}

Blood for platelet function testing was collected for testing as described in section 2.2.2. Genotyping for of CYP2C19*2 and CYP2C19*17 were performed on isolated genomic DNA using the RFLP and sanger sequencing techniques as described in section 4.2.7.

\subsubsection{End points and definitions}

The primary endpoint was a composite of major adverse cardiovascular events (MACE) including death, nonfatal myocardial infarction, nonfatal ischaemic stroke and stent thrombosis.

\subsubsection{Major adverse cardiac event (MACE)}

Death encompassed all-cause mortality including cardiovascular death. Acute MI was defined using the third universal definition of myocardial infarction (289). This included the detection of a significant hs-TnT rise and the presence of symptoms suggestive of myocardial ischaemia, new or presumed new ST-segment-T wave changes or new LBBB, development of new pathological $Q$ waves, or imaging showing new myocardial loss. Evaluation of definite stent thrombosis was performed according to the Academic Research Consortium criteria (345). Acute ischaemic cerebrovascular accident (CVA) was defined as signs of CVA confirmed with imaging studies. 


\subsubsection{Bleeding}

The Bleeding Academic Research Consortium Definition for Bleeding (BARC) (346) was used to define bleeding. Major bleeding was determined as fatal bleeding or BARC type 3 or type 4 CABG bleeds. Minor bleeding included BARC type 2 bleeds.

Type 4 CABG-related bleeds include perioperative intracranial bleeding, reoperation for bleeding after closure of sternotomy, transfusion of $\geq 5 \mathrm{U}$ of whole blood or packed red blood cells within 48 hour period and a chest tube output $\geq 2 \mathrm{~L}$ within a 24 hour period. Type 3 bleeds include any overt bleeding with the need for transfusion or a drop in hemoglobin of more than $30 \mathrm{~g} / \mathrm{L}$ due to the bleed. Cardiac tamponade, bleeding requiring surgical intervention, bleeding requiring intravenous vasoactive agents, intracranial hemorrhage or intraocular bleeds are also included.

Type 2 bleeds are any overt bleeding events that do not fit the criteria for type 3 or 4 but still require either nonsurgical, medical intervention by a healthcare professional or hospitalisation.

\subsubsection{Hospital readmission}

All hospital readmissions were noted and notes were called on each visit to further classify events.

\subsubsection{Statistics}

Categorical variables are expressed as frequencies and percentages. Univariate analysis of demographic and biomarker variables as categories with outcome data was performed using Chi-Squared tests. Multivariate analysis of significant univariate predictors of outcome was performed using a multinominal logistic regression analysis. Cut-points derived from ROC curve analysis was determined as the multiplate $A U$ value with the highest sensitivity and highest specificity-1. Regression analysis, ChiSquared tests and ROC curve analysis were performed using PASW 18.0 (IBM, NY, USA), all other analysis and graphs were generated using GraphPad prism 5 (GraphPad Software Inc., CA, USA). 


\subsection{Results}

All patients in the study underwent coronary angiography and their management is summarised in Table 5-1. Radial access was the predominant route of access used in $79 \%$ of cases. $\mathrm{PCl}$ was performed in $53.8 \%$ of patients at the time of their index angiogram and the remaining patients being either medically managed (34\%) or referred for CABG (12.2\%).

Table 5-1 Coronary angiogram procedure variables

\begin{tabular}{|l|c|}
\hline & $\mathrm{N}=294$ \\
\hline Underwent coronary angiography & $294(100)$ \\
\hline Radial access & $234(79)$ \\
\hline Stenosis $>50 \%$ in $\geq 1$ coronary artery & $255(86.7 \%)$ \\
\hline Management & \\
\hline Medical Management & $100(34)$ \\
\hline PCl & $158(53.8)$ \\
\hline CABG & $36(12.2)$ \\
\hline
\end{tabular}

\subsubsection{Patient outcome}

At 1 year follow up, 31 patients (10.5\%) experienced MACE. This included 10 deaths (3.4\%), of which 9 were identified to be due to cardiovascular causes and 1 due to cancer. Nonfatal myocardial infarction occurred in 17 patients $(5.8 \%)$ and ischaemic CVA in $6(2 \%)$ patients. The rate of stent thrombosis was relatively low, occurring in 4 patients (1.4\%) during the follow-up period.

Bleeding ( $\geq$ BARC 2 ) occurred in 40 patients (13.6\%) at 1 year follow up. There were 9 major bleeds (3.0\%) made up of 5 BARC type $3(1.7 \%)$ and 4 BARC type 4 CABG bleeding (1.4\%). The remaining 31 patients (10.5\%) had minor bleeds classified as BARC type 2 bleeds. Of note, 14 of the non-CABG bleeds were related to coronary angiogram access site complications.

A further 83 patients were readmitted to hospital who did not experience MACE or bleeding (28.2\%). Of interest, 12 patients were admitted with unstable angina (4.0\%) 
and 5 of these patients underwent revascularization (1.7\%). A further 7 patients were admitted with congestive heart failure (2.4\%).

\subsubsection{ROC curve analysis of residual platelet reactivity and outcome}

To assess the predictive value of residual platelet reactivity to identify patients at increased risk of MACE and bleeding, ROC curve analysis was conducted. Residual platelet reactivity was found to be a moderate predictor of MACE with an area under the curve of $0.631(0.542-0.744)$ (Figure $5-1)$. The ROC curve demonstrated the lack of a single cut-point that was predictive of MACE risk, including the previously established $47 \mathrm{AU}$. A cut point of $35 \mathrm{AU}$ for HOTPR was derived from the platelet reactivity value with the highest sensitivity and smallest loss of specificity (Table 5-2), which has a sensitivity of 0.710 and specificity of 0.51 .

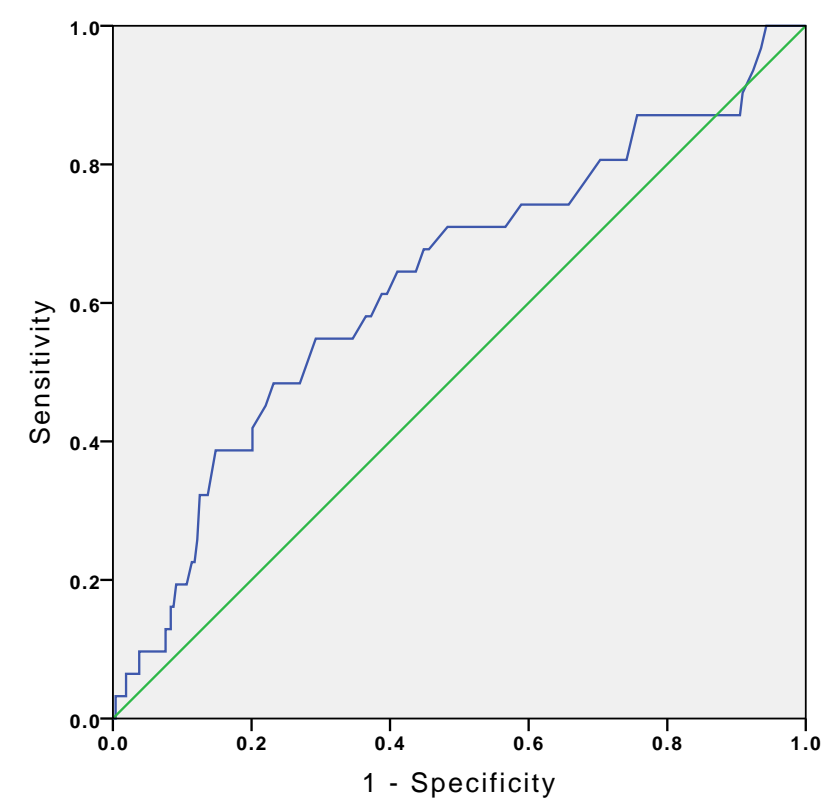

Figure 5-1 ROC curve analysis of residual platelet reactivity and MACE 
Table 5-2 ROC curve coordinates

\begin{tabular}{|c|c|c|}
\hline MEA value & Sensitivity & Specificity \\
\hline 31 & 0.743 & 0.463 \\
\hline 32 & 0.743 & 0.482 \\
\hline 33 & 0.743 & 0.494 \\
\hline 34 & 0.743 & 0.514 \\
\hline 35 & 0.743 & 0.529 \\
\hline 36 & 0.686 & 0.553 \\
\hline 37 & 0.686 & 0.560 \\
\hline 38 & 0.657 & 0.572 \\
\hline 39 & 0.657 & 0.576 \\
\hline 40 & 0.657 & 0.591 \\
\hline 41 & 0.657 & 0.599 \\
\hline 43 & 0.629 & 0.623 \\
\hline 45 & 0.600 & 0.646 \\
\hline 46 & 0.543 & 0.661 \\
\hline 47 & 0.514 & 0.677 \\
\hline
\end{tabular}

With the new HOTPR cut-point of $35 \mathrm{AU}$, an additional 42 patients were included in the HOTPR group compared to categorizing using the $47 \mathrm{AU}$ cut-point (Figure 5-2). This change resulted in a HOTPR rate of $51.7 \%$ compared to $37.5 \%$ with the previous classification. 


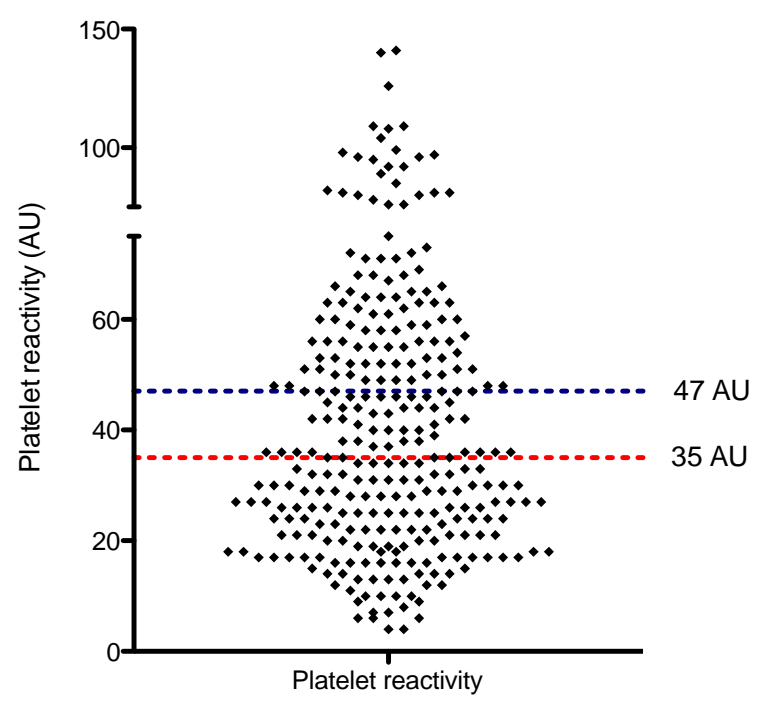

Figure 5-2 Residual platelet reactivity and HOTPR cut-points

Residual platelet reactivity as measured by the Multiplate (AU) plotted for each patient. The blue dotted line represents the 47 AU HOTPR cut-point as described by Sibbing et al (310). The red dotted line represents the 35 AU HOTPR derived from ROC curve analysis in this study.

In ROC curve analysis of major bleeding (Figure 5-3) there was no relationship between residual platelet reactivity and outcome with an area under the curve of 0.492. ROC curve analysis was then performed with all bleeding as the outcome (Figure 5-4) and no relationship was observed between residual platelet reactivity and bleeding The area under the curve was 0.499 indicating residual platelet reactivity is not a significant predictor of bleeding, major or minor.

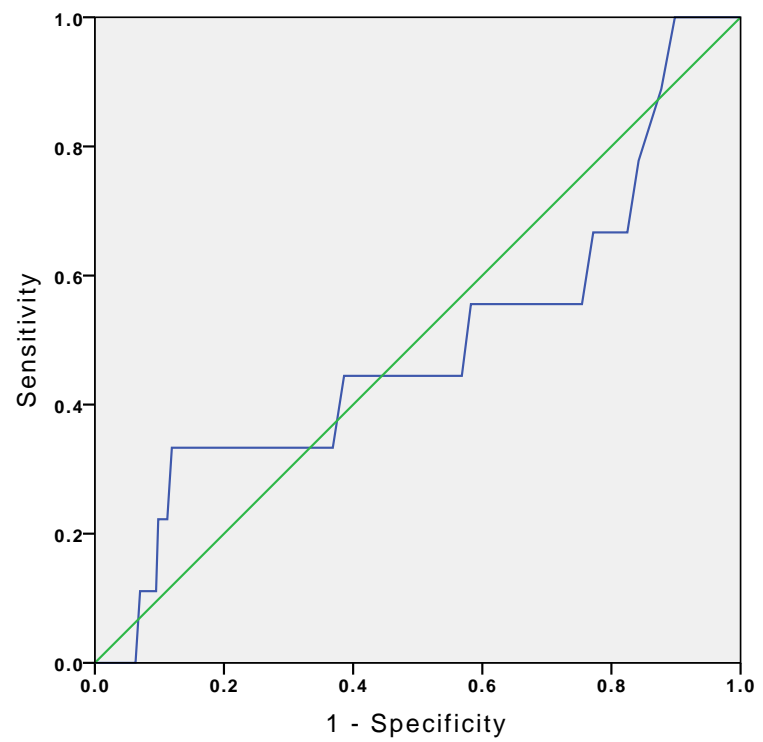

Figure 5-3 ROC curve analysis of residual platelet reactivity and major bleeding 


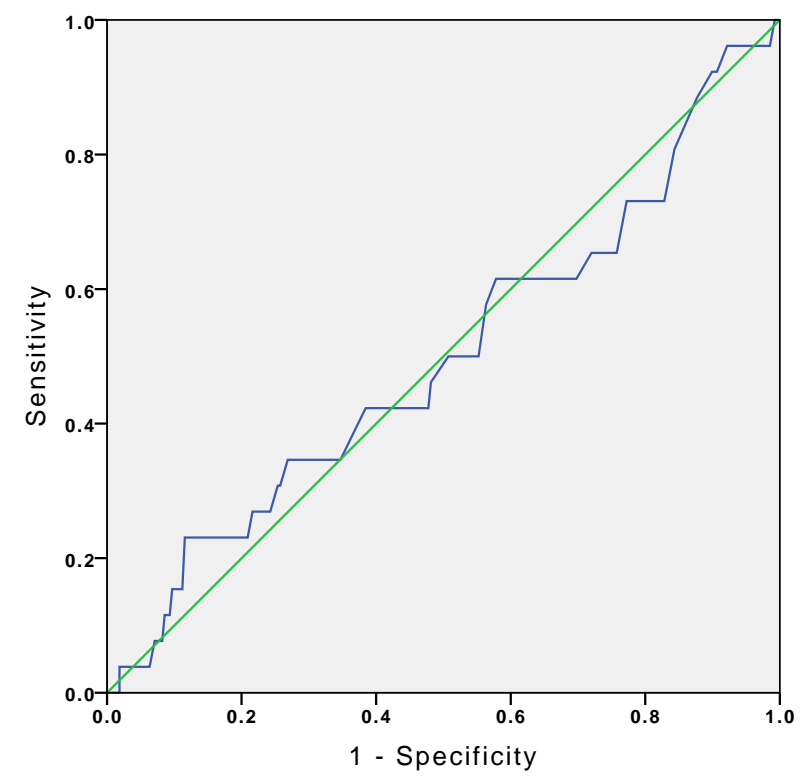

Figure 5-4 ROC curve analysis of residual platelet reactivity and all bleeding

\subsubsection{Predictors of MACE}

Univariate analysis was conducted to assess whether the factors that were predictive of high residual platelet reactivity were also predictive of outcome (Figure 5-5). HOTPR with the cut-point of $35 \mathrm{AU}$ resulted in a 2.5 fold increase risk of $\operatorname{MACE}(2.5,1.1$ - 5.6, $p=0.023$ ) this is compared to the historical HOTPR cut-point of $47 \mathrm{AU}$ which identified patients with a 2.2 fold increased risk $(2.2,1.1-4.7, p=0.034)$. Diabetes was also found to be a significant predictor of $\operatorname{MACE}(3.2,1.5-6.8, p=0.002)$ whereas obesity $(\mathrm{BMI}>30,0.9,0.4-2.1, \mathrm{p}=0.9)$, LOF allele $(0.74,0.31-1.7, \mathrm{p}=0.4)$, homozygous LOF allele $\left(* 2 /{ }^{*} 2\right)$ carriers $(1.06,0.12-7.0, p=0.9)$, low clopidogrel dose $(1.3,0.6-$ 2.8, $p=0.4)$ and high platelet count $(1.2,0.4-3.7, p=0.7)$ were not significantly correlated with increased MACE risk. In multivariate analysis both diabetes and HOTPR >35 AU were found to be significant independent predictors of MACE (Figure 5-6). 


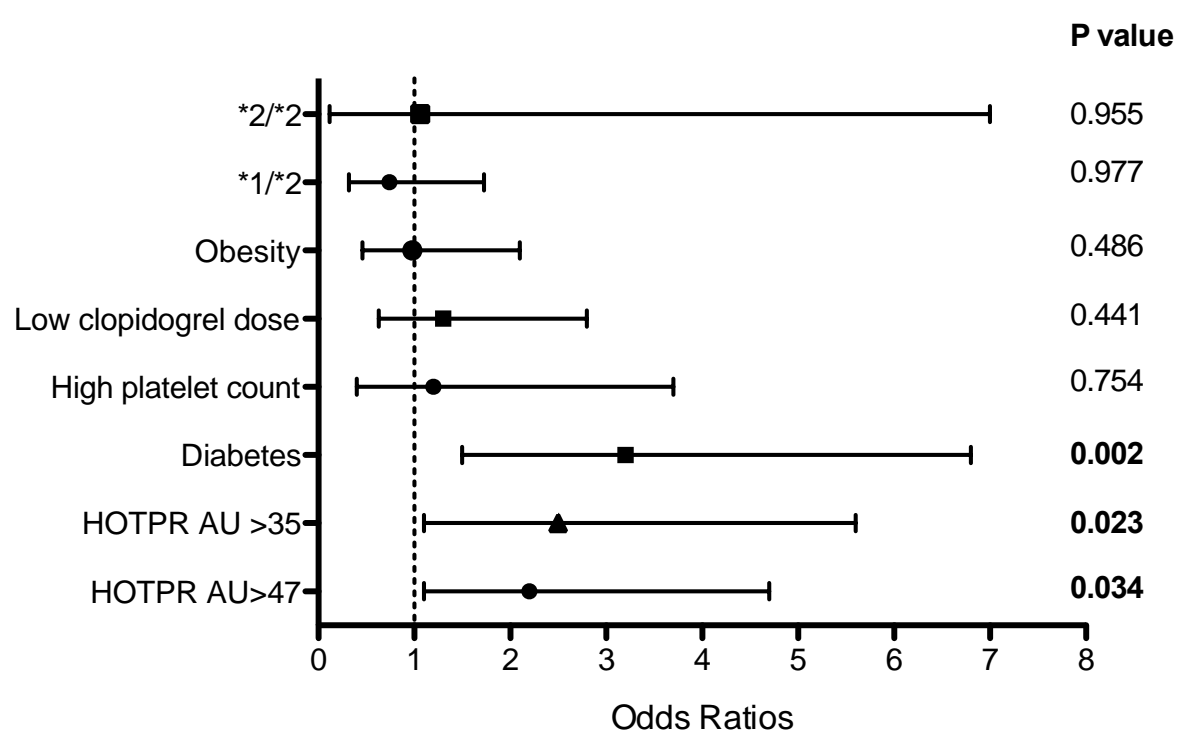

Figure 5-5 Univariate predictors of MACE

Clinical and demographic variables represented as odds ratios for predictors of MACE at 1 year and $95 \%$ confidence intervals.

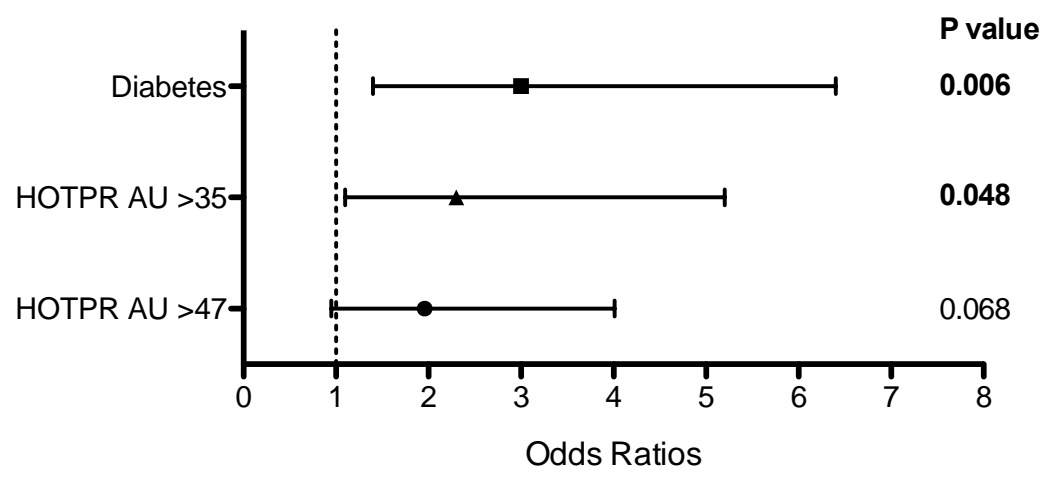

Figure 5-6 Multivariate predictors of MACE

Clinical and demographic variables represented as odds ratios for predictors of MACE at 1 year and $95 \%$ confidence intervals. 


\subsubsection{Predictors of bleeding}

Univariate analysis was also conducted to determine predictors of bleeding. None of the factors analysed were found to be predictive of bleeding (Figure 5-7). These included femoral access $(1.8,0.8-3.6, \mathrm{p}=0.155)$, low weight $(\mathrm{BMI}<20,0.6,0.2-1.5$, $p=0.2)$, low platelet count $\left(<150 \times 10^{9} / L, 1.5,0.4-4.8, p=0.5\right)$, diabetes $(0.9,0.4-$ 2.0), high clopidogrel dose $(1.4,0.7-2.8, p=0.3)$, heterozygous $\left({ }^{*} 1 /{ }^{*} 17\right)$ GOF allele $(0.9,0.2-3.7, p=0.7)$ and patients homozygous $\left({ }^{*} 17 / * 17\right)$ for the GOF allele $(0.6,0.1$ $-5.0, p=0.6$ ). As previously demonstrated in ROC curve analysis, residual platelet reactivity was not predictive of bleeding.

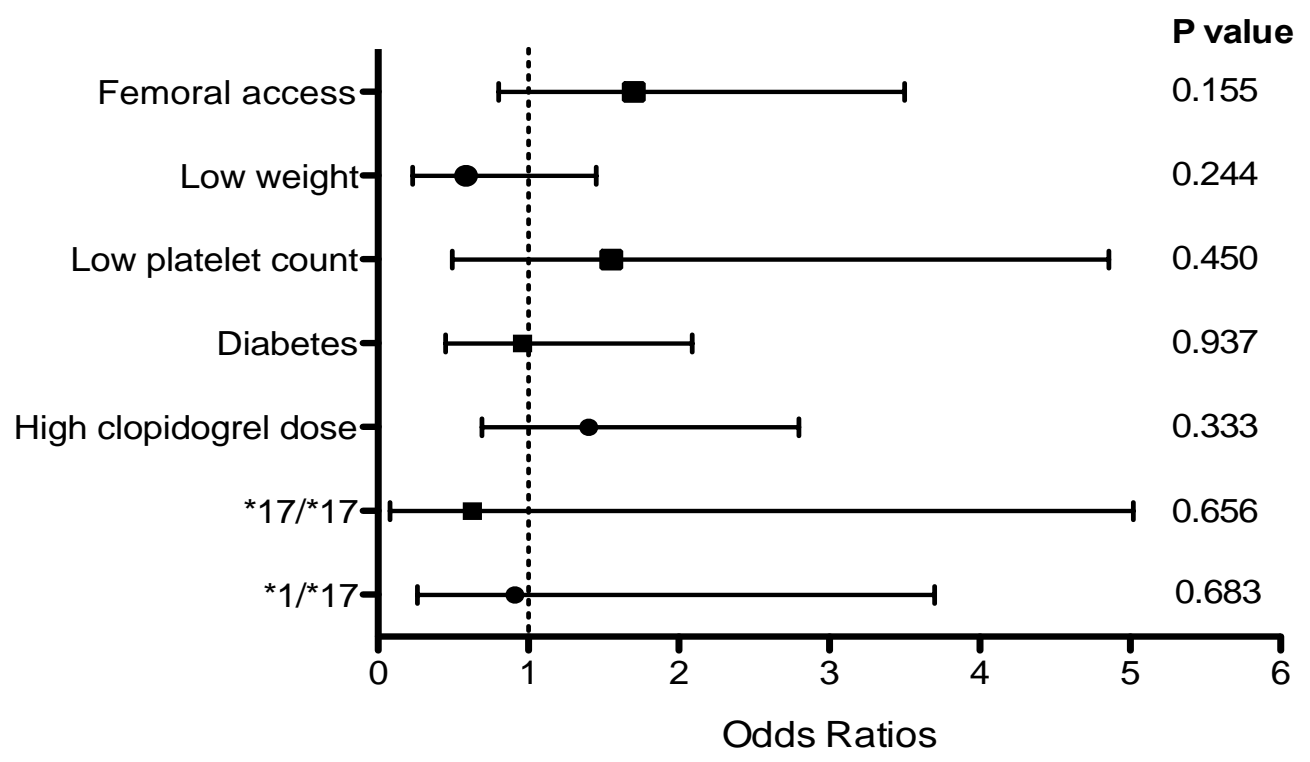

Figure 5-7 Univariate predictors of bleeding

Clinical and demographic variables represented as odds ratios for predictors of bleeding at 1 year and $95 \%$ confidence intervals. 


\subsubsection{Subgroup analysis of $\mathrm{PCl}$ cohort}

Further analysis was performed in the $\mathrm{PCl}$ subgroup to investigate whether residual platelet reactivity was a stronger predictor in this population. Procedure variables for the cohort are summarized in Table 5-3.

Table 5-3 PCl procedure variables

\begin{tabular}{|l|c|}
\hline & $\mathrm{N}=158$ \\
\hline Average number of lesions treated & $1.3 \pm 0.5$ \\
\hline Average number of vessels treated & $1.2 \pm 0.4$ \\
\hline Average number of stents & $1.4 \pm 0.7$ \\
\hline Proportion of stents implanted DES & $76 \%$ \\
\hline Average stent length $(\mathrm{mm})$ & $24.4 \pm 13.6$ \\
\hline Average stent diameter $(\mathrm{mm})$ & $3.0 \pm 0.9$ \\
\hline
\end{tabular}

Within the $\mathrm{PCl}$ cohort 15 patients experienced MACE, which resulted in a similar MACE rate $(9.7 \%)$ compared to the total population (10.5\%). MACE events in the $\mathrm{PCl}$ cohort were comprised of 4 cardiovascular deaths (2.5\%), 8 acute MI (5.2\%), 2 ischaemic CVA (1.3\%) and 4 stent thrombosis (2.6\%).

ROC curve analysis of residual platelet reactivity and MACE in the $\mathrm{PCl}$ cohort displayed a modest relationship with a similar area under the curve of $0.649(0.470-0.828)$ to the ROC curve in the total population. A HOTPR cut-point was also derived from the platelet reactivity value with the highest sensitivity and smallest loss of sensitivity (Table 5-4), which equated to an AU of 43 , sensitivity of 0.733 and specificity of 0.594 . 


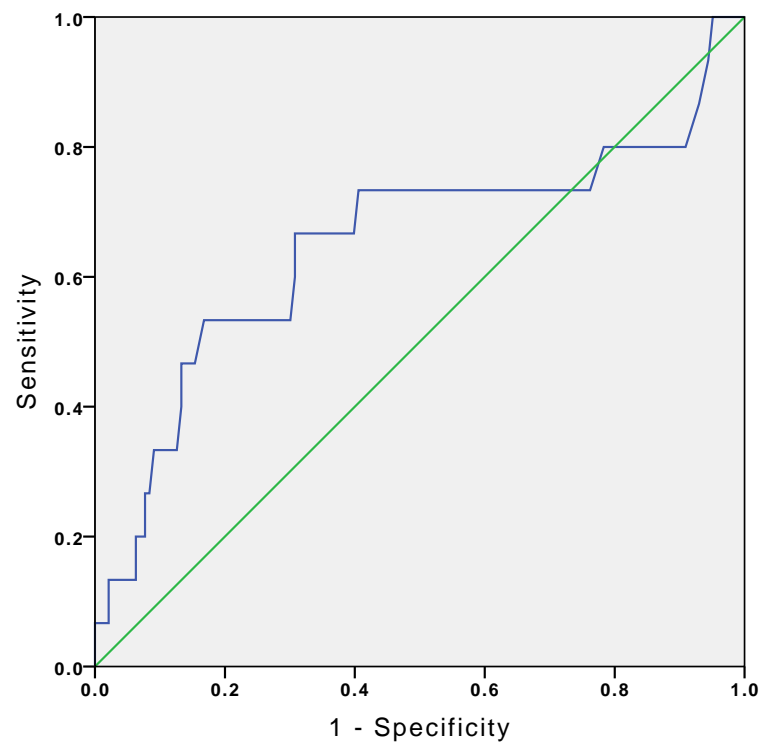

Figure 5-8 ROC curve analysis of residual platelet reactivity and MACE in PCI subgroup

Table 5-4 PCI ROC curve coordinates

\begin{tabular}{|l|l|l|}
\hline MEA value & Sensitivity & Specificity \\
\hline 33 & 0.733 & 0.434 \\
\hline 34 & 0.733 & 0.469 \\
\hline 35 & 0.733 & 0.490 \\
\hline 36 & 0.733 & 0.538 \\
\hline 37 & 0.733 & 0.545 \\
\hline 38 & 0.733 & 0.559 \\
\hline 39 & 0.733 & 0.566 \\
\hline 40 & 0.733 & 0.573 \\
\hline 41 & 0.733 & 0.580 \\
\hline 43 & 0.733 & 0.594 \\
\hline 45 & 0.667 & 0.601 \\
\hline 46 & 0.667 & 0.629 \\
\hline 47 & 0.667 & 0.657 \\
\hline
\end{tabular}

Univariate analysis to investigate the predictors of MACE in the $\mathrm{PCl}$ cohort produced similar results as seen in the full population (Figure 5-9). Diabetes was associated with an increased risk of MACE $(6.4,2.1-19.6, p=0.001)$ as well as the HOTPR $\geq 43$ AU cutpoint $(3.8,1.2-12.5, p=0.02)$ and $\operatorname{HOTPR} \geq 47 \mathrm{AU}(3.2,1.1-9.8, p=0.03)$ in the $\mathrm{PCl}$ 
cohort. The HOTPR >35AU cut-point was no longer a significant predictor of MACE in the $\mathrm{PCl}$ cohort as well as genotype, obesity, high platelet count and low clopidogrel dose. Evident from the wide confidence intervals displayed for the univariate predictors, conducting subgroup analysis reduces the number of MACE events and therefore statistical power. Multivariate analysis was conducted and found diabetes continued to be a significant predictor of $\operatorname{MACE}(5.1,1.6-16.2, p=0.005)$ whereas both HOTPR cut-points of $47 \mathrm{AU}$ or $43 \mathrm{AU}$ were not independent predictors (Figure 5-10).

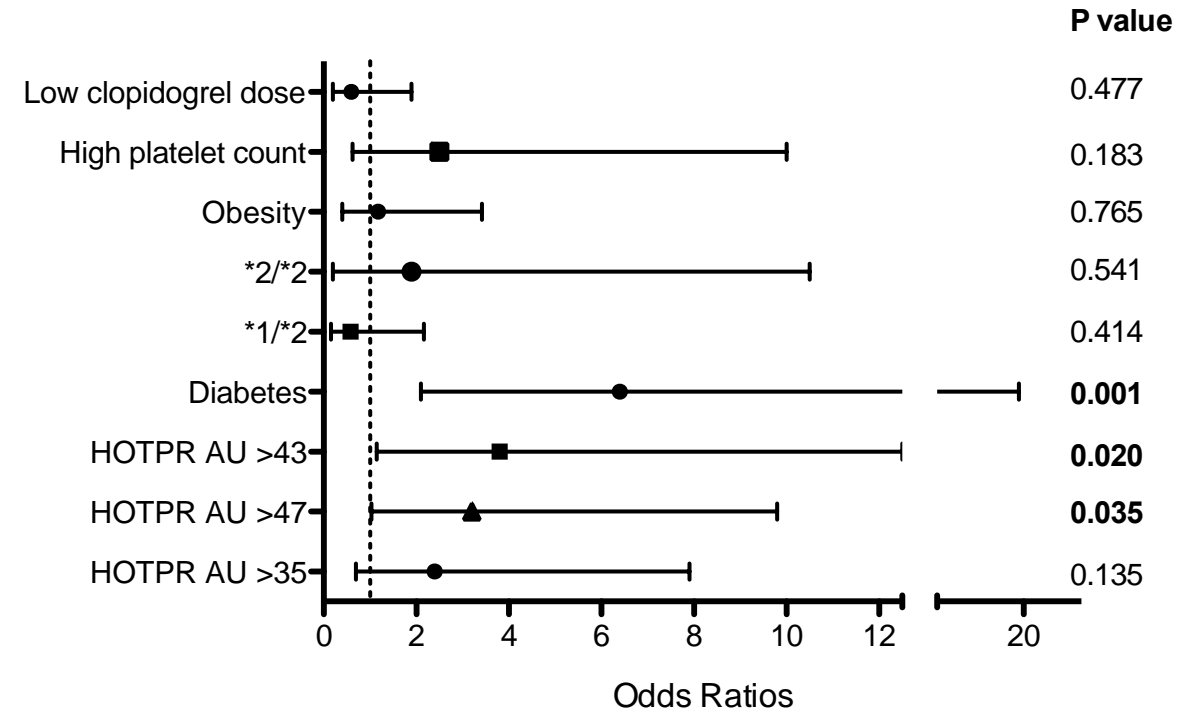

Figure 5-9 Univariate predictors of MACE in the $P C l$ subgroup

Clinical and demographic variables represented as odds ratios for predictors of MACE at 1 year and $95 \%$ confidence intervals.

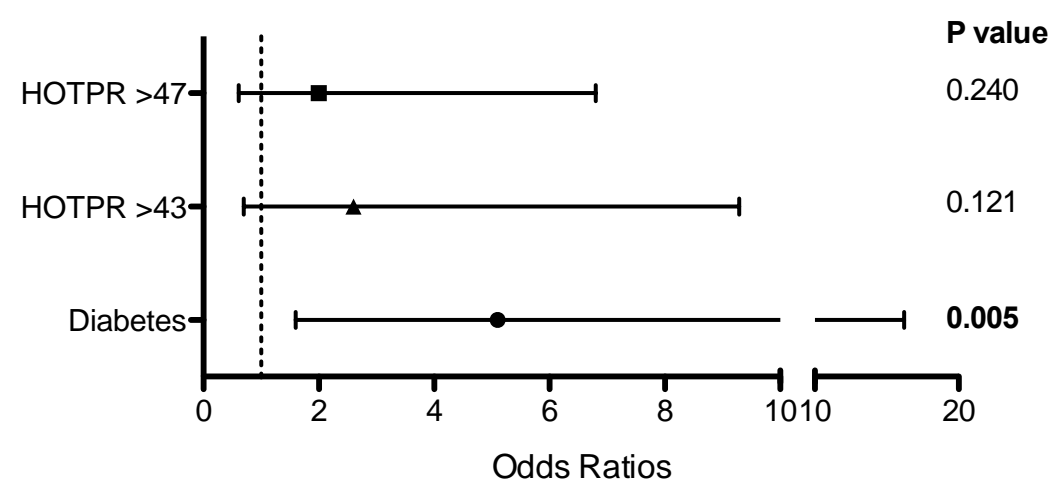

Figure 5-10 Multivariate analysis of univariate MACE predictors in the PCI
subgroup

Clinical and demographic variables represented as odds ratios for predictors of MACE at 1 year and $95 \%$ confidence intervals. 


\subsection{Discussion}

This was the first study to investigate the relationship between phenotype, genotype and other clinical risk factors with outcome and identify which factors were independent predictors in the same cohort. We found the HOTPR phenotype and not the LOF genotype predicted MACE at 1 year. Furthermore, diabetes, which was shown to be a predictor of the HOTPR phenotype, was also a strong predictor of MACE independent of HOTPR. Bleeding rates were low in this cohort and were not predicted by low residual platelet reactivity or other clinical factors.

Several studies including those conducted by Gurbel et al, Price et al, Sibbing et al and Matetzky et al., have demonstrated the HOTPR phenotype as measured by various platelet function tests is a strong predictor of MACE in CAD patients $(181,182,185$, 347). Similarly, meta-analyses of outcome trials predominantly made up of patients undergoing $\mathrm{PCl}$ on clopidogrel, have demonstrated an increased risk of MACE in patients homozygous and heterozygous for the LOF allele (232). However, our study suggests that phenotype and not genotype predicts risk of MACE in an ACS population. As previously identified in Chapter 4, carriage of the LOF allele has a moderate impact on high residual platelet reactivity and is only 1 of many factors that influence this phenotype. We also investigated whether other factors that influenced residual platelet reactivity were also predictors of MACE. Patients with diabetes are known to have poor cardiovascular outcomes compared to non-diabetic cardiac patients (246) and as our study and others have shown diabetes is a significant contributor to HOTPR. However, to our knowledge this is the first study to demonstrate that HOTPR and diabetes are independent predictors of MACE risk. Therefore platelet function testing could offer additional information on a patients risk beyond a patients' diabetes status.

We also examined the other identified HOTPR predictors and found obesity (BMI >30), low clopidogrel dose and high platelet count did not predict MACE in our population. We did not investigate the predictive value of an exhaustive list of demographic and 
clinical factors in this cohort, as we did not have the power to make multiple comparisons in this cohort.

This study also highlighted that defining HOTPR by a single cut-point on a continuum of platelet reactivity is somewhat problematic. Undeniably, high residual platelet reactivity is associated with an increased risk of MACE, but where the optimal cutpoint is remains unknown. The ROC curve derived in the whole population identified a HOTPR cut-point of $35 \mathrm{AU}$, which is significantly lower than the HOTPR definition used previously and would result in half of the patients being classified at higher risk. Furthermore, the ROC curve derived in the $\mathrm{PCl}$ cohort sub analysis was different again, although confidence intervals where wide, with an optimal cut-point at $43 \mathrm{AU}$, which is more in line with the established multiplate cut-point (47 AU) determined by Sibbing and co (282). The issues with identifying the optimal cut-point are due to several factors that either increase the variability in the measurement of platelet reactivity or skew the risk of MACE.

One issue in the current study is the relatively small population size, which reduces the level of confidence around the derived cut-point compared to the Sibbing group, which derived their cut-point from a much larger cohort of 1608 patients. Furthermore, as we have demonstrated in chapter 2 , the assay itself has an inherent variability of $10 \%$ in the measurement of platelet reactivity. In addition to this, several pre-measurement factors such as anticoagulant and time can compound this variability and widen the confidence around a platelet function measurement identifying someone at risk. The adverse events we include to identify the optimal cutpoint are not homogenous. The degree in which high residual platelet reactivity is driving the risk of death, stroke, $\mathrm{Ml}$ and stent thrombosis will vary and including these events as a composite endpoint may reduce our power to define the most sensitive cut-point. However, there are also limitations to only using a single outcome such as stent thrombosis at 30 days used by Sibbing et al. The resulting applicability of a cutpoint defined only by stent thrombosis may be inadequate as a tool to personalize antiplatelet therapy and reduce adverse events. There also exists variability in the risk of patients experiencing adverse events that we perform platelet function testing in. 
The Sibbing study (344) derived their HOTPR threshold in a mixed population of stable and acute CAD patients where their risk of MACE would vary considerably. Our study exclusively examined an acute population as these patients have the highest risk of recurrent thrombotic events and therefore may benefit the most from adequate antiplatelet therapy.

Bleeding complications in patients with ACS have been associated with an increased risk of subsequent adverse outcomes including $\mathrm{MI}$, stroke, stent thrombosis and death (348-352). Recognition of this relationship between bleeding, mortality and morbidity has lead to a new emphasis on identifying those at risk of bleeding and strategies to minimize bleeding. In our cohort residual platelet reactivity did not predict major bleeding or all bleeding and no other significant predictors of major or all bleeding were identified. Although the link between HOTPR and ischaemic events is well established, the relationship between low platelet reactivity and bleeding is less clear. Although a number of previous studies in patients undergoing $\mathrm{PCl}$ have demonstrated an association between low platelet reactivity and bleeding $(338,353-355)$ this relationship was not observed in a number of other large-scale platelet function studies including the POPULAR, GRAVITAS and ARCTIC studies (287, 356-358).

The rate of major bleeding in the cohort was low (3\%). The high rate of radial access and the low rates of GP IIb/IIla antagonist use in our study are likely to have contributed to this. The standardized definition recently proposed by the Bleeding Academic Research Consortium was used in the study (346). Previous observational studies and clinical trials have used a wide variety of other bleeding definitions making comparisions of bleeding rates across trials difficult (346). As a result of the low bleeding rate our study was underpowered to look at the relationship between bleeding and platelet reactivity, genotype or clinical factors. Major bleeding in our study included a combination of non-CABG and CABG related bleeding. The role that residual platelet function plays in these different types of bleeding might vary but because of the low number of events we were not able to examine these subgroups. 
Based on the observations linking HOTPR with an increased risk of ischaemia and LOWPR with an increased risk of bleeding, the concept of a therapeutic window for platelet reactivity has been proposed $(206,281)$. Following this observation, several studies have aimed to improve patient outcome by targeting the therapeutic window using platelet function testing or genotyping. Two small studies by Bonelle et al. randomised patients undergoing $\mathrm{PCl}$ to a guided antiplatelet using the VASP assay or standard antiplatelet therapy. Those who were found to have HOTPR in the guided arm were given an additional 600mg loading of clopidogrel. This treatment was successful in reducing on treatment platelet reactivity and also decreased the rate of early stent thrombosis without an increase in major bleeding compared to the nonguided approach $(283,284)$. The selective addition of GPIIb/IIla antagonists in patients with HOTPR as identified with the VerifyNow assay, has also been shown to improve patient outcomes without increasing bleeding in the 3T/2R trial (359).

However, the three largest randomized trials using platelet function testing to tailor antiplatelet therapy failed to demonstrate any benefit from this approach. There were several issues with the study design of these trials that may have contributed to the negative results. The GRAVITAS trial was the first large scale trial $(n=2214)$ that utilized the VerifyNow P2Y12 assay to identify patients with HOTPR and subsequently doubled clopidogrel dosing (287). High-dose clopidogrel treatment was ineffective in reducing the 6-month composite ischemic event occurrence (cardiovascular death, nonfatal $\mathrm{MI}$, and stent thrombosis); both treatment groups had an unexpectedly low event rate (2.3\%). Similarly, the ARCTIC study enrolled 2440 patients undergoing PCI to receive guided therapy with the VerifyNow or non-guided therapy. Several different treatments were available to clinicians in the guided arm including reloading with clopidogrel (600mg), switching to prasugrel or administration of a GPIIb/IIla inhibitor during $\mathrm{PCl}$. The study was also neutral with no improvement in clinical outcomes for patients receiving guided therapy compared to standard clopidogrel therapy without monitoring (287). The TRIGGER-PCI trial, which compared prasugrel vs. clopiodgrel in patients with HOTPR as identified by the VerifyNow, was stopped by the steering committee prematurely after randomization of 423 patients (out of the planned 2150) due to an interim analyses indicating a lower than expected incidence in the primary 
endpoint. Given the low rate of ischemic events the clinical utility of this strategy could not be demonstrated (288).

The limitations in these clinical trials are due to the treatment strategy, the study population and the method of identified high-risk patients. Firstly the treatment strategy utilized in the GRAVITAS study of single re-loading $(600 \mathrm{mg})$ and double maintenance therapy $(150 \mathrm{mg}$ ) was not potent enough to overcome HOTPR in a large proportion of those tested. A dramatic $40 \%$ of patients in the guided therapy arm with HOTPR remained non-responders after receiving additional clopidogrel. In the ARCTIC study, prasugrel was a treatment option for clinicians, although this treatment only became available towards the end of the enrolment period and of the cohort only 3.3\% were switched to the more potent $\mathrm{P} 2 \mathrm{Y} 12$ inhibitor. The large majority of patients (80.2\%) received increased doses of clopidogrel, which has been shown to be ineffective in lowering platelet reactivity in those with HOTPR. Both pharmacokinetic studies and large clinical trials have demonstrated ticagrelor and prasugrel therapy, result in lower on treatment platelet reactivity levels and a decrease in MACE compared to clopidogrel $(203,217,360,361)$. Furthermore, in chapter 3 we demonstrated the treatment of patients with HOTPR with prasugrel resulted in a marked reduction in residual platelet reactivity in all patients. Therefore it is not surprising that this inadequate personalized therapy of additional clopidogrel, failed to show clinical benefit.

The populations in all of these studies were largely stable patients undergoing elective $\mathrm{PCl}$ and high-risk patients (STEMI) were excluded. The recent ADAPT-DES registry indicates that intensified antiplatelet therapy may not benefit low-risk patients but might improve patient outcomes in high-risk patients; high risk ACS or patients with a high risk for stent thrombosis; diabetes, multiple stents and multivessel disease (364). Therefore exclusion of high-risk patients in these large trials could have contributed to the lack of benefit in the personalized therapy arm. The GRAVITAS and TRIGGER$\mathrm{PCl}$ trials were also statistically underpowered to a point where TRIGGER-PCI was stopped prematurely and GRAVITAS has a much lower primary endpoint rate than expected (calculated as $5 \%$ vs. observed $2.3 \%$ ). This could also be due to the low-risk 
population that was recruited. It has been suggested that as for studies with tailored therapy of prasugrel or ticagrelor, a much higher sample size (at least 17,000 patients) would be required to show statistical differences (365).

Lastly, the assay and cut-point used to identify HOTPR needs to be considered in these studies. The three trials employed the VerifyNow P2Y12 assay with a threshold of 235 PRU to identify HOTPR. Subsequent analysis of the GRAVITAS trial hypothesized that the original threshold may have been too high with a clustering of events above and below the threshold and conversely, a PRU $<208$ was strongly associated with an event free survival at 60 days (364). This adjusted threshold of 208 PRU was also confirmed in the ADAPT-DES, a study investigating ischaemic outcomes and clinically relevant bleeding at 1-year follow-up, in more than 8500 patients treated with clopidogrel and undergoing $\mathrm{PCl}$. A PRU>208 resulted in a 2.5 fold increase risk of stent thrombosis and myocardial infarction (364). Therefore the method used to identify patients at risk may not have been sensitive enough.

Several key issues around the measurement of platelet reactivity and personalisation of antiplatelet therapy remain and require further investigation before platelet function testing can be considered for clinical use. As this study has demonstrated, finding the optimal cut-point for HOTPR is challenging but key to identifying those who will benefit the most. As identified in chapter 2, the standardised measurement of platelet reactivity is important to reduce analytical variation and the use of a wide sampling to testing time frame may have significantly reduced the sensitivity and specificity of the cut-point derived by Sibbing et al (344). The appropriate population to test residual platelet reactivity is also unknown. As previously discussed, there is limited evidence to show personalised antiplatelet therapy is beneficial in stable CAD patients from the ADAPT-DES registry. However, previous studies in ACS populations have largely focused on platelet function testing in $\mathrm{PCl}$ patients and the predictive value of HOTPR in medically managed populations or patients undergoing CABG need to be established. An extension of this study to address these limitations is planned to identify the optimal cut-point for HOTPR using a consistent Multiplate methodology in a larger cohort of 700 ACS patients. 
In New Zealand there is a significant increase in the use of ticagrelor, due to government funding of this therapy in ACS patients from 1 July 2013. As the antiplatelet therapy landscape evolves, the use of platelet function testing to tailor therapy must also evolve. Although ticagrelor was shown to be superior to clopidogrel at reducing MACE in ACS patients it also increases the risk of non-CABG related major bleeding (365). Therefore, instead of the routine use of ticagrelor in all patients who present with an ACS, it may be possible to use platelet function testing to identify a group of patients who would have adequate platelet inhibition and protection from ischaemic events on clopidogrel without an increased bleeding risk. This strategy may lead to equivalent outcomes at a considerably lower cost, although such a strategy has not yet been proven to work.

\subsubsection{Limitations}

As the study was observational and all aspects of clinical management were at the consultant's discretion, we cannot exclude the possible influence of other medications or clinical and procedure variables on our results. The study was underpowered to investigate the relationship between multiple demographic and clinical factors with the risk of MACE and bleeding. It would have been interesting to determine the presence of other clinical risk factors that could potentially inform a modified risk score in ACS patients. However, to conduct a study that included a large number of patients with 1 year follow up in the time frame of the thesis was not possible.

\subsubsection{Conclusion}

In this study, HOTPR phenotype and not CYP2C19*2 genotype is a predictor of ischaemic risk in an ACS population. However, identifying the optimal cut-point for classifying HOTPR remains complex and challenging. Diabetes was also found to be a strong predictor of ischaemic risk. Importantly this study demonstrated that HOTPR and diabetes independently predict MACE at one year. In the PCl cohort, HOTPR remained a predictor of MACE at one year on univariate but not on multivariate analysis. The bleeding rate was low in our cohort and low residual platelet reactivity 


\section{CHAPTER 5}

was not predictive of these events. Identifying the optimal cut-point for classifying HOTPR and whether residual platelet reactivity can be used improve the predictive power of existing risk stratification models or personalise antiplatelet therapy requires further investigation. 


\section{Chapter 6: Platelet reactivity, biomarkers and outcome following vascular surgery}




\subsection{Introduction}

Postoperative $\mathrm{MI}$ and major cardiac complications occur in more than $4 \%$ of the patients with either an established diagnosis of CAD or risk factors for CAD, who undergo major non-cardiac surgery (366). Patients undergoing vascular surgery appear to be at particularly high risk of perioperative cardiac complications such as death and nonfatal myocardial infarction. Previous studies suggested that myocardial injury (troponin elevation) occurred in $21-24 \%$ of patients undergoing vascular surgery and that this elevation of post-operative troponin levels is strongly associated with an increased mortality (367-369). Treatment with aspirin has been shown to substantially reduce this risk (59). However, as seen in the previous chapters, the biological response to antiplatelet therapy varies significantly between ACS patients and it is logical to assume that this variance would also exist in vascular patients.

In ACS patients our previous studies have demonstrated that residual platelet reactivity is predictive of outcome. To date, the relationship between residual platelet reactivity and outcomes in patients undergoing major elective vascular surgery has not been established, despite their high risk for cardiac complications. Furthermore, the utility of other biomarkers such as high sensitivity troponin (hs-TnT), C-reactive protein (CRP) and NT-proBNP (brain naturetic protein), that have shown to be highly predictive of poor outcome in a CAD population, have not been thoroughly examined in vascular patients. There remains a lack of evidence based risk stratification tools to apply in vascular populations that can identify those at increased risk of ischaemic and bleeding events both perioperatively and long term.

Therefore the aim of this chapter was to:

1. To investigate the relationship between residual platelet reactivity on aspirin as well as the cardiac biomarkers hs-TnT, NT-proBNP and CRP with adverse cardiovascular and bleeding outcomes in patients undergoing major vascular surgery.

2. To examine the relationship between these biomarkers with perioperative and long-term outcomes. 


\subsection{Methods}

\subsubsection{Study Population}

Patients presenting to Wellington Regional Hospital for elective major vascular surgery between April 2011 and December 2012 were eligible for inclusion in the study if they were adequately pre-treated with aspirin. Elective major vascular surgery included carotid endarterectomy, open abdominal aortic aneurysm repair and infrainguinal procedures including femoral-popliteal bypass and femoral enadarterectomy. Adequate pre-treatment was defined as chronic therapy with aspirin $(100 \mathrm{mg} /$ day for more than 7 days) or loading with aspirin $\geq 300 \mathrm{mg}$ at least 2 hours prior to enrolment. Exclusion criteria included a recent ACS (<3 months), platelet count less than $100 \mathrm{x}$ $10^{9} / \mathrm{L}$, known platelet function disorder, treatment with an antiplatelet agent other than aspirin, acute limb ischaemia, symptomatic AAA, current active infection and those unable to give informed consent. The study was reviewed and approved by the Central Regional Ethics Committee (NTX-11-03-018).

\subsubsection{Data Collection}

Patient demographics, clinical characteristics, medications including antiplatelet therapy, clinical management and procedural variables were obtained prospectively from review of the medical records. All aspects of clinical management were at the discretion of the attending physicians. All ischaemic and bleeding complications occurring during the hospital admission were recorded. Patients were also contacted by phone at 30 days and 1 year by a cardiology research nurse, to assess clinical outcomes. Where necessary, review of case notes and contact with the general practitioner was performed to further classify clinical outcomes.

\subsubsection{Study design, ECG and blood collection}

The study was a prospective observational study of patients undergoing major elective vascular surgery. Electrocardiograms (ECGs) and venous blood samples were collected from all patients at baseline, before surgery and on postoperative days 1 and 2 . 
Further blood samples for hs-TnT measurement and electrocardiograms were performed where clinically indicated. Blood for platelet function testing was collected as described in section 2.2.2. Blood for high sensitivity troponin T (hs-TnT), C-reactive protein (CRP) and N terminus pro-B type Naturetic Peptide (NT-ProBNP) was collected into a serum-separator collection tube (BD Diagnostics, NJ, USA)

\subsubsection{Platelet function testing and biomarker analysis}

Platelet function testing was performed as described in section 2.2.2. Whole blood based impedence aggregometry was performed using the Multiplate analyser using $0.5 \mathrm{mM}$ arachadonic acid to stimulate platelets and test the thromboxane pathway. The upper and lower quintiles of residual platelet reactivity were empirically defined as HOTPR and LOWPR respectively.

All hs-TnT, NT-proBNP and CRP measurements were performed in the Wellington Regional Hospital Medical Laboratory using the Cobas CE modular system (Roche Diagnostics, Basel, Switzerland). A hs-TnT level $\geq 14 \mathrm{ng} / \mathrm{L}$ was considered elevated. Elevated NT-proBNP was age adjusted and a positive value was defined as $>53 \mathrm{pmol} / \mathrm{L}$ for age $<50$ years, $>106 \mathrm{pmol} / \mathrm{L}$ for age $50-75$ years and $>212 \mathrm{pmol} / \mathrm{L}$ for age $>75$ years. The lower limit of detection for CRP was $3 \mathrm{mg} / \mathrm{L}$. For CRP the upper quintile was emperically defined as an elevated value.

\subsubsection{End points and definitions}

\subsubsection{Primary end point}

The primary endpoint was major adverse cardiovascular events (MACE) at one year. MACE was defined as a composite of cardiovascular death, nonfatal myocardial infarction, nonfatal ischaemic stroke, periprocedural myocardial injury and graft occlusion at one year. Secondary endpoints included MACE at 30 days, bleeding at 30 days and 1 year and rehospitalisation. 


\subsubsection{Ischaemic events}

Cardiovascular death was considered as any death with a demonstrable cardiovascular cause or any death that is not clearly attributable to a noncardiovascular cause. Acute myocardial infarction was defined using the third universal definition of myocardial infarction (289), including a detection of a significant hs-TnT rise in the presence symptoms suggestive of myocardial ischaemia, new or presumed new ST-segment-T wave changes or new LBBB, development of new pathological Q waves, or imaging evidence of new myocardial loss. An isolated significant elevation in hs-TnT of more than $50 \%$ compared to baseline hs-TnT levels and an absolute rise of at least $>20 \mathrm{ng} / \mathrm{L}$ with no other evidence of myocardial ischaemia was considered as periprocedural myocardial injury. Acute ischaemic cerebrovascular accident (CVA) was defined as signs of CVA confirmed with imaging studies. Graft Occlusion was defined as any graft occlusion confirmed by duplex ultrasound or angiography.

\subsubsection{Bleeding events}

Major bleeding was defined as fatal bleeding, intracranial bleeding, drop in postoperative hemoglobin of $>50 \mathrm{~g} / \mathrm{L}$ perioperatively, transfusion of $\geq 2$ units of Red Blood Cells ( $\mathrm{RBC}$ ), bleeding requiring reoperation or bleeding requiring readmission to hospital.

\subsubsection{Statistical analysis}

In this chapter a different statistical approach has been used to investigate the relationship between biomarkers, clinical factors and patient outcome compared to the statistical analysis in the ACS cohort. In the preceding chapters the number of comparisons has been deliberately limited to reduce the probability of false positive results. However, in the current study, due to a limited recruitment (see limitations section 6.5), a limited comparison approach would potentially lead to a greater problem with false negative findings (372). Therefore, the multiple comparisons are displayed as forest plots for completeness and potential incorporation into larger meta-analyses and to also display the variability due to the reduced power in the 
cohort. Interpretation of all of these results needs to be undertaken with care, mindful of the large confidence intervals observed within these forest plots.

Categorical variables are expressed as frequencies and percentages. Continuous variables not normally distributed following D’Agostino \& Pearson normality testing are expressed as median and IQR. Surgical variables were found to be normally distributed and are expressed as mean and standard deviation and analsyed using 1 way ANOVA. Univariate analysis of demographic and biomarker variables as categories with outcome data was performed using Chi-Squared tests. Multivariate analysis of significant univariate predictors of outcome was performed using a multinominal logistic regression analysis. Regression analysis, Chi-Squared tests and ROC curve analysis were performed using PASW 18.0 (IBM, NY, USA), all other analysis and graphs were generated using GraphPad prism 5 (GraphPad Software Inc., CA, USA). 


\subsection{Results}

\subsubsection{Demographics}

During the study period 106 patients were enrolled in the study. Of those enrolled 6 patients were subsequently excluded for the following reasons, 3 patients decided to have their surgery performed in a private hospital due to the waiting time in the public hospital, 2 patients presented acutely prior to their scheduled elective surgery and one was initially planned to have an open AAA but subsequently the vascular team decided to use an endovascular approach. One year follow-up was completed in all of the remaining 100 patients and the baseline demographics are displayed in Table 6-1.

Table 6-1 Patient baseline demographics

\begin{tabular}{|l|l|}
\hline & $\mathrm{N}=100$ \\
\hline Age (years) & $72(45-88)$ \\
\hline BMle, $\mathrm{n}$ & 61 \\
\hline Ethnicity, $\mathrm{n}$ & $27(16-38)$ \\
\hline European & \\
\hline Maori and Pacific Islander & 91 \\
\hline Other & 7 \\
\hline Risk Factors, $\mathrm{n}$ & 2 \\
\hline Hypertension & \\
\hline Dyslipidaemia & 86 \\
\hline Diabetes & 80 \\
\hline Current Smoker & 33 \\
\hline Previous Smoker & 15 \\
\hline Previous TIA/Stroke & 59 \\
\hline Previous Vascular Surgery & 31 \\
\hline Ischaemic Heart Disease & 30 \\
\hline Laboratory data & 43 \\
\hline Creatinine (umol/L) & \\
\hline Platelet Count (109/L) & $96(47-270)$ \\
\hline Haemoglobin (g/L) & $229(124-479)$ \\
\hline
\end{tabular}


Admission medications were recorded for each patient (Table 6-2). As per the inclusion criteria, all patients were on aspirin therapy. There was also a high administration of statin therapy (86\%) and the majority of patients were on a form of hypertension medication.

Table 6-2 Admission medications

\begin{tabular}{|l|l|}
\hline Medication & $\mathrm{N}=100$ \\
\hline Aspirin & 100 \\
\hline Beta Blocker & 50 \\
\hline Statin & 86 \\
\hline ACE inhibitor & 44 \\
\hline Diuretic & 25 \\
\hline Warfarin & 3 \\
\hline Nitrate & 7 \\
\hline Calcium Channel Blocker & 37 \\
\hline
\end{tabular}

Baseline measurements of the biomarkers, platelet reactivity, hs-TnT, NT-proBNP and CRP were performed in every patient and there was a wide range of baseline values for all 4 biomarkers (Table 6-3, Figure 6-1). Platelet reactivity ranged from 2-142 AU with the upper quintile ( $>24 \mathrm{AU}$ ) and lower quintile ( $<7 \mathrm{AU})$ empirically defined as HOTPR and LOWPR respectively. Hs-TnT also varied greatly at admission, with the highest value at $152 \mathrm{ng} / \mathrm{L}$ and $35 \%$ of patients had a baseline hs-TnT that was elevated above the upper limit of normal ( $\geq 14 \mathrm{ng} / \mathrm{L}$ ). NT-proBNP was determined in all patients and elevated levels were determined by adjusting for age, resulting in the identification of 13 patients with elevated levels. CRP was detectable in $41 \%$ of patients at baseline (>3 mg/L). As nearly $59 \%$ of patients had an undetectable level of CRP, elevated levels were also empirically defined as the upper quintile of measurements (>6 mg/L). 
Table 6-3 Baseline measurement of biomarkers

\begin{tabular}{|l|l|}
\hline Multiplate platelet reactivity $(\mathrm{AU})$ & $16.5(2-142)$ \\
\hline Hs-TnT (ng/L) & $9.5(3-153)$ \\
\hline Elevated hs-TnT & $35 \%$ \\
\hline NT-proBNP (pmol/L) & $33.5(1-521)$ \\
\hline Elevated NT-proBNP & $13 \%$ \\
\hline CRP (mg/L) & $3(3-46)$ \\
\hline Elevated CRP & $20 \%$ \\
\hline
\end{tabular}
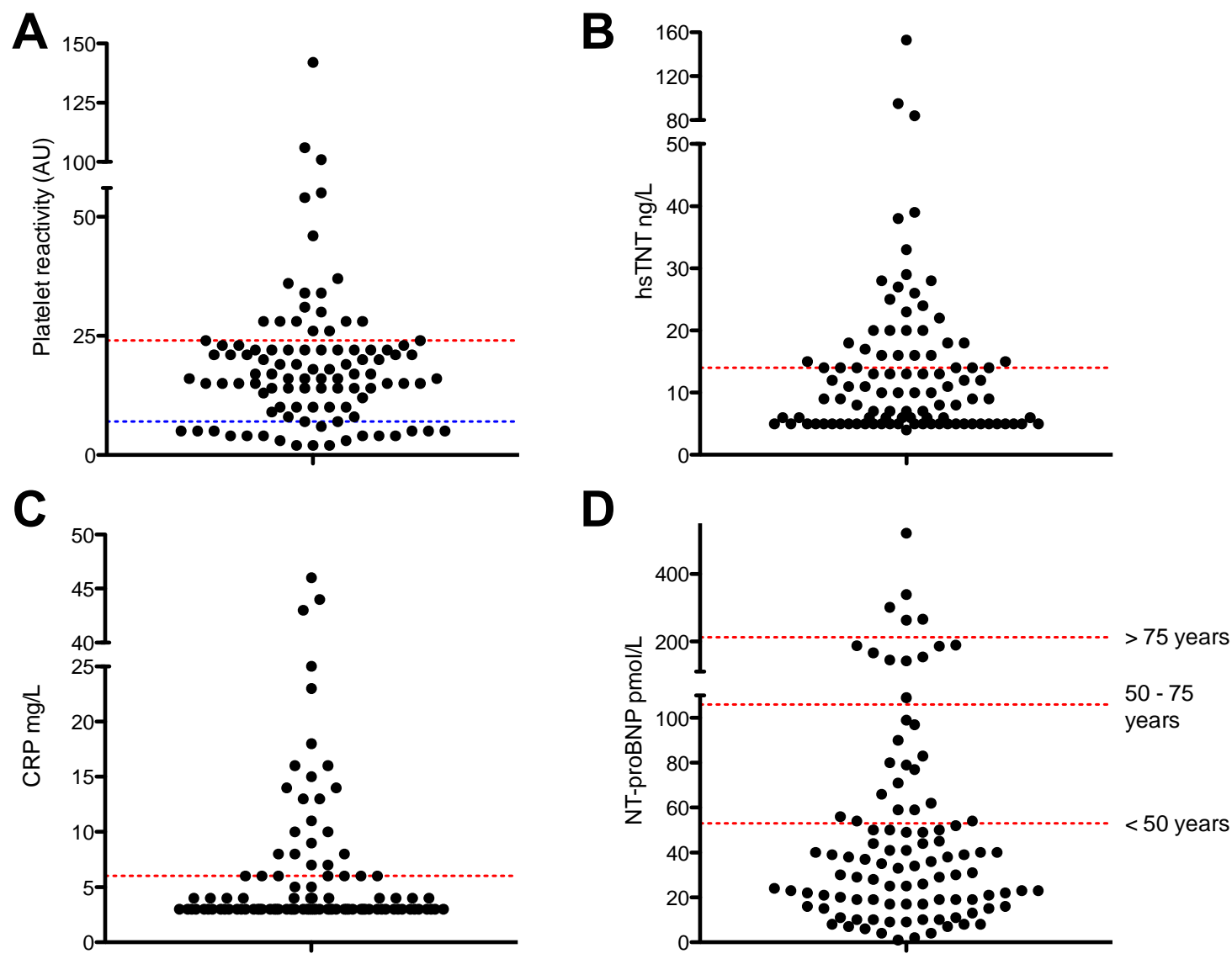

Figure 6-1 Variability of baseline biomarkers

Baseline residual platelet reactivity (A), hs-TnT (B), CRP (C) and NT-proBNP (D) were measured in all patients. The red and blue dotted line for residual platelet reactivity represents the upper ( $>24 \mathrm{AU})$ and lower quintiles $(>7 \mathrm{AU})$ respectively $(\mathrm{A})$. The red dotted line for hs-TnT represents the upper limit of normal $(>14 \mathrm{ng} / \mathrm{L})$ and an undetected level was lower than $4 \mathrm{ng} / \mathrm{L}$. The red dotted line for CRP represents the upper quintile $(>6 \mathrm{mg} / \mathrm{L})$ and an undetected level was lower than $3 \mathrm{mg} / \mathrm{L}$. The three dotted lines for NT-proBNP represent the age-adjusted cutpoint for elevated levels. 
The type of anesthesia, duration of surgery and estimated blood loss was recorded during surgery by the anesthetist and vascular surgeon for each patient (Table 6-4). The majority of patients received a general anesthetic (84\%). Epidural alone was used solely in patients undergoing infra-inguinal surgery and some patients undergoing an AAA received a combined approach. The mean procedure time across all surgeries was 161 mins \pm 69 and differed significantly across the three surgery sub groups ( $p<$ 0.0001 ). The longest surgery times were in the AAA group (224 mins \pm 51 ) and shortest in patients undergoing carotid endarterectomy surgery (108 mins \pm 31 ). Similarly, estimated blood loss was significantly higher in the group undergoing AAA at $1045 \mathrm{~mL}$ \pm 973 compared to infra-inguinal surgery $(236 \mathrm{~mL} \pm 462)$ or carotid endarterectomy (41 mL $\pm 103, p<0.0001)$.

Table 6-4 Surgery details

\begin{tabular}{|l|l|l|l|l|l|}
\hline & $\begin{array}{l}\text { All patients } \\
\mathrm{n}=100\end{array}$ & $\begin{array}{l}\text { Carotid } \\
\mathrm{n}=37\end{array}$ & $\begin{array}{l}\text { AAA } \\
\mathrm{n}=22\end{array}$ & $\begin{array}{l}\text { Infra-inguinal } \\
\mathrm{n}=41\end{array}$ & $\begin{array}{l}\text { P } \\
\text { value }\end{array}$ \\
\hline Anesthesia & & & & & \\
\hline General & 84 & 37 & 15 & 32 & \\
\hline Epidural & 4 & 0 & 0 & 4 & \\
\hline Combined & 12 & 0 & 7 & 5 & 0.0001 \\
\hline $\begin{array}{l}\text { Procedure time } \\
\text { (mins) }\end{array}$ & $161 \pm 69$ & $108 \pm 31$ & $224 \pm 51$ & $174 \pm 68$ & $\begin{array}{l}< \\
0.0001\end{array}$ \\
\hline $\begin{array}{l}\text { Blood loss } \\
\text { during surgery } \\
\text { (mL) }\end{array}$ & $342 \pm 662$ & $41 \pm 103$ & $\begin{array}{l}1045 \pm \\
973\end{array}$ & $236 \pm 462$ & \\
\hline
\end{tabular}

\subsubsection{Perioperative measurement of hs-TnT}

As $35 \%$ of patients had elevated hs-TnT levels at baseline, a significant hs-TnT rise perioperatively was determined by the change in hs-TnT between baseline and postoperative measurements. As displayed in Figure 6-2A, 17 patients had a significant rise in hs-TnT compared to baseline. However, as depicted in Figure 6-2B, where patients did not have a significant rise in hs-TnT post operatively compared to baseline, if hsTnT were only measured on day 1 or day $2,31 \%$ of the total population would appear to have elevated levels which would indicate a possible acute $\mathrm{MI}$ and lead to further unnecessary investigations. 
A

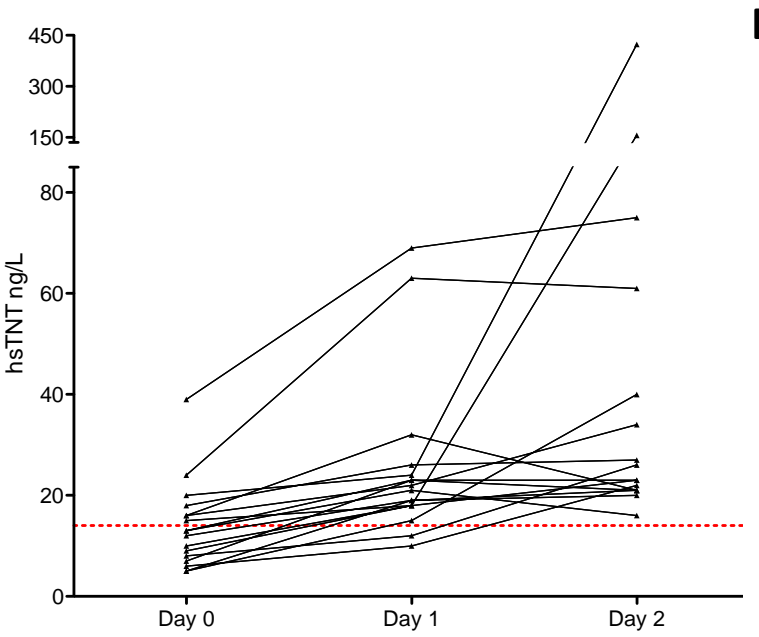

B

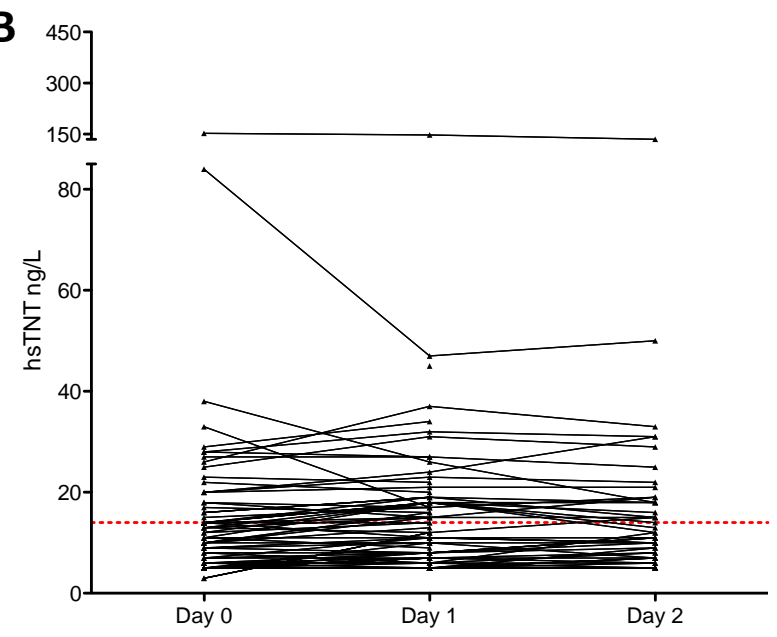

Figure 6-2 Hs-TnT measurement perioperatively

$\mathrm{Hs}$-TnT measurements at baseline (Day 0 ) and Day 1 and 2 post-operatively in patients with a significant rise in hs-TnT (A) and patients who did not have a significant rise compared to baseline (B). The red dotted line represents the upper limit of normal (14ng/L).

\subsubsection{Patient outcomes following vascular surgery}

To look at predictors of patient outcome following vascular surgery, data was collected in-hospital, at 30 days and at 1 year following surgery. Overall 34 patients experienced MACE with a total of 39 events occurring in these patients. Furthermore, major bleeding occurred in 29 patients with a total of 32 events occurring in these patients. Patient outcome data was also divided into short ( 0 - 30 days post surgery) and long-term ( 30 days -1 year post surgery) time periods as the nature of events short term differed compared to events occurring after 30 days.

\subsubsection{Short term outcomes}

During the time period from surgery up to day 30, 23 patients experienced MACE. Some patient's experienced more than one event, these included 9 periprocedural myocardial injury, 8 acute $\mathrm{MI}$ events, 1 CVA and 5 graft occlusions. The majority of these events occurred on day 1 or day 2 post-operatively (Figure 6-3). During the same time period, 29 patients experienced major bleeding events. In the 24 patients, 15 patients received transfusions of $\geq 2$ RBC units, 3 patients had hematomas requiring reoperation, 8 patients had a haemoglobin drop of more than $50 \mathrm{~g} / \mathrm{L}$ and 5 patients 
were readmitted with major bleeding. Of the patients who had bleeding or experienced MACE in this time period, 13 patients experienced both outcomes. Post discharge there was a further 12 patients who re-presented to hospital with varied problems including; wound infection, gastroenteritis, fever, exacerbation of COPD, non cardiac chest pain and bowel issues.

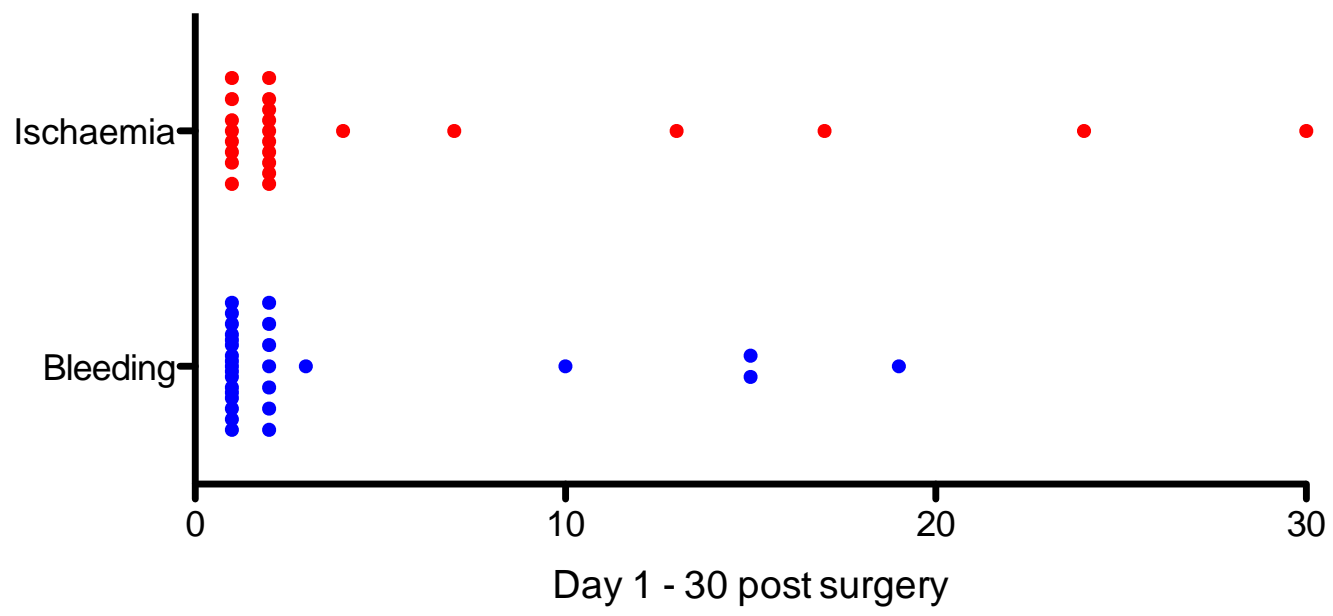

Figure 6-3 Short term timing of events post surgery

Each dote represents an individual MACE (red) or bleeding (blue) event during the patients hospital admission. Mean and SEM are displayed.

\subsubsection{Long term outcomes}

In the time period between 30 days and 1 year post surgery, a further 12 patients experienced MACE. Five patients died in the follow up period, 1 cardiovascular death and 4 documented as non-cardiac deaths, 5 patients were also readmitted with graft occlusion as confirmed by duplex. Acute Ml occurred in 2 patients and 2 patients were readmitted with an ischaemic CVA. There was a consistent incidence of MACE events over this time period (Figure 6-4). During this period, 3 patients experienced major bleeding complications resulting in a readmission to hospital. A further 23 patients had re-admissions to hospital during the follow up period. These included 16 elective vascular procedures (angioplasty or surgery) for progression of vascular disease and 7 other minor elective procedures. An additional 16 patients presented to ED with varying complaints, but these presentations did not require admission. 


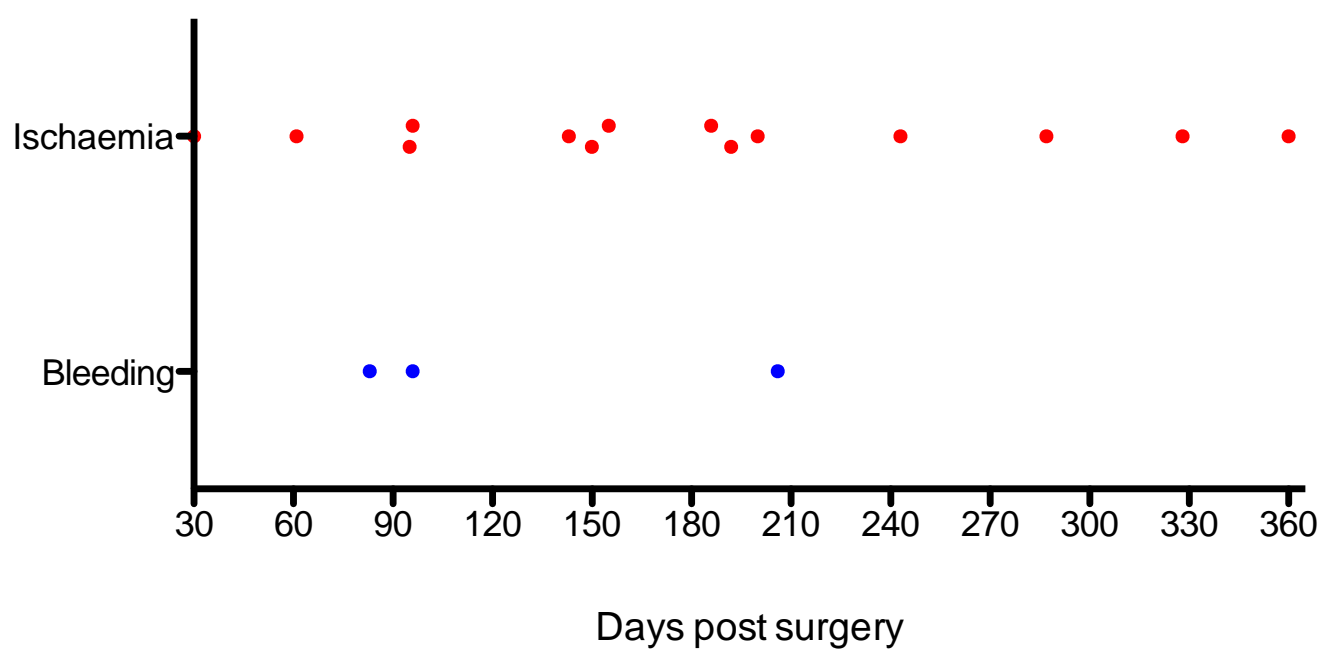

Figure 6-4 Timing of events from 30 days to 1 year post discharge

Each dote represents an individual MACE (red) or bleeding (blue) event between 30 days and 1 year post discharge from hospital.

\subsubsection{Predictors of adverse outcomes in vascular patients}

\subsubsection{Adverse outcomes at 1 year}

Several clinical factors predicted an increased risk of MACE at 1 year in univariate analysis (Figure 6-5). These included a $\mathrm{BMI}<25$ (3.4 (1.5-8.3), $\mathrm{p}=0.005)$, elevated baseline hs-TnT (3.6 $(1.5-8.6), p=0.003)$, elevated CRP $(2.3(1.0-5.3), p=0.047)$ and renal impairment $(2.3(0.98-5.4), p=0.05)$. Of these factors an elevated baseline hs$\operatorname{TnT}(3.7(1.4-10.1), p=0.008)$ and $\mathrm{BMI}<25$ (4.1 (1.5 - 11.1), $\mathrm{p}=0.005)$ were found to be independent predictors of MACE at 1 year in multivariate analysis (Figure 6-6). HOTPR and elevated NT-proBNP did not significantly correlate with MACE at 1 year. 


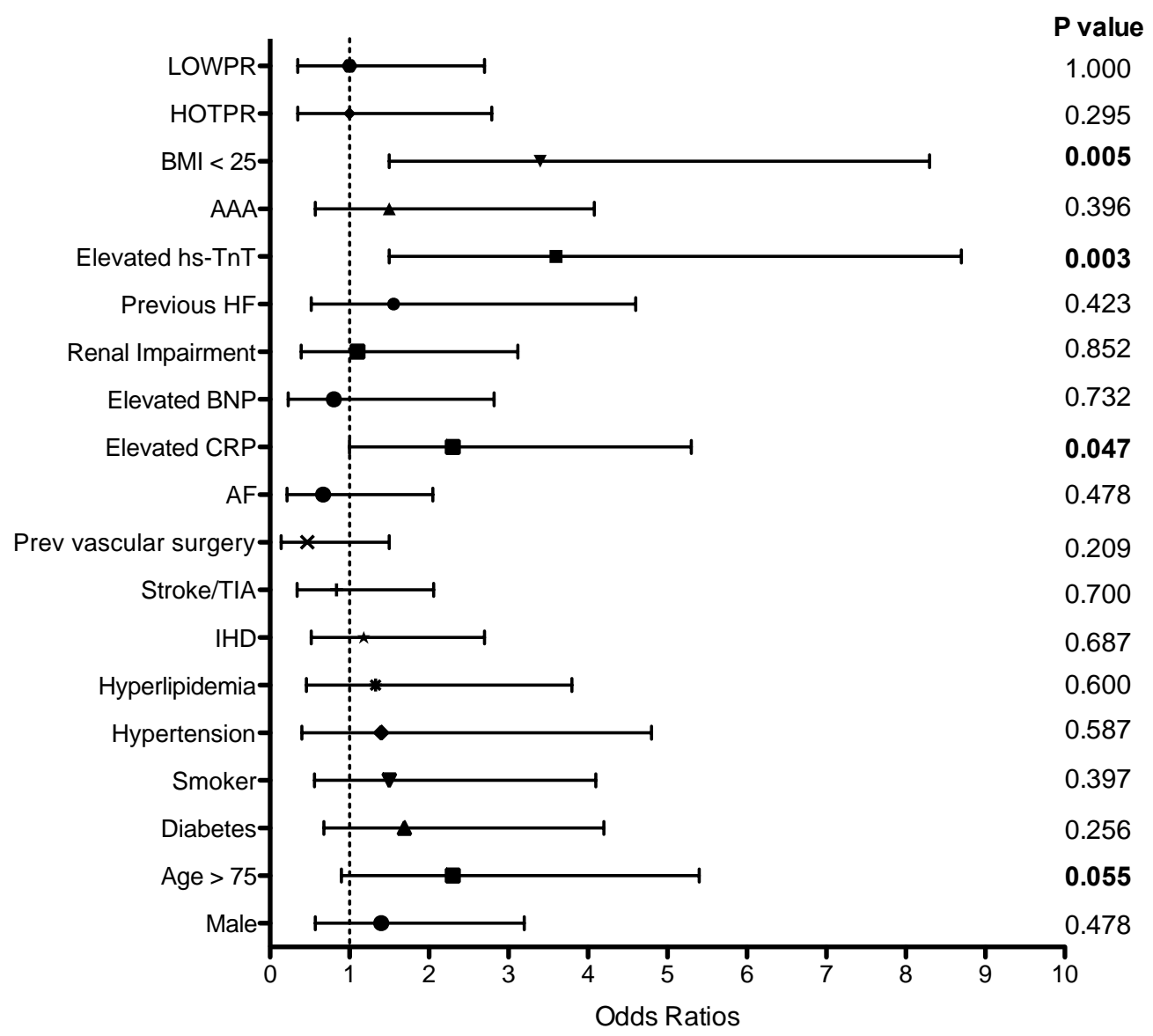

Figure 6-5 Predictors of MACE at 1 year

Clinical and demographic variables represented as odds ratios for predictors of MACE at 1 year and $95 \%$ confidence intervals.

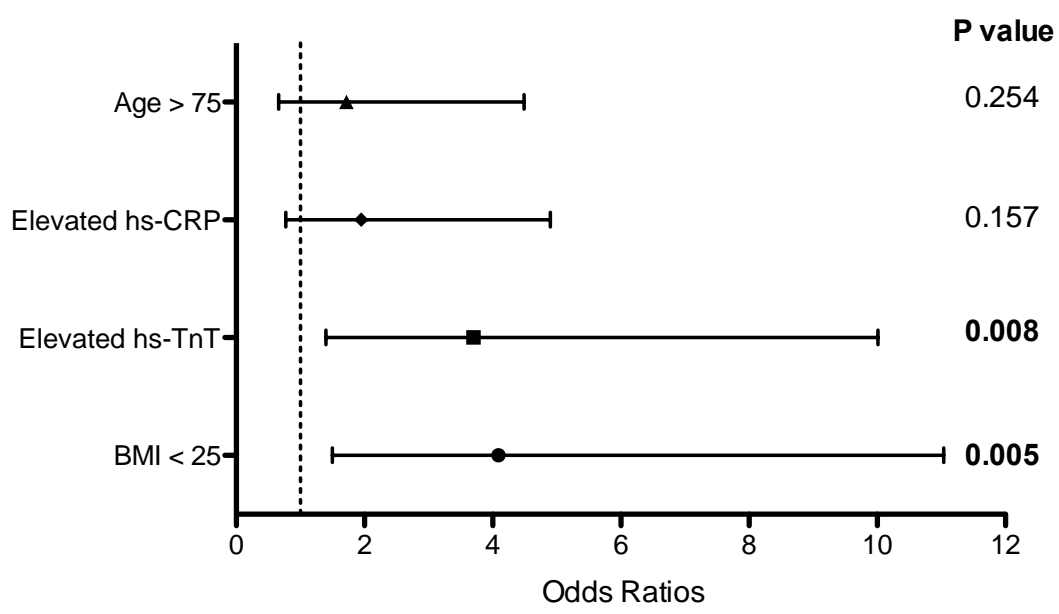

Figure 6-6 Multivariate analysis of predictors of all MACE

Clinical and demographic variables represented as odds ratios for predictors of MACE at 1 year and $95 \%$ confidence intervals. 
ROC curve analysis was also conducted to investigate if there was any relationship between residual platelet reactivity and MACE at 1 year (Figure 6-7). The ROC curve displayed no relationship with an area under the curve 0.482 and no discernable cutpoint that would identify patients at increased risk of MACE.

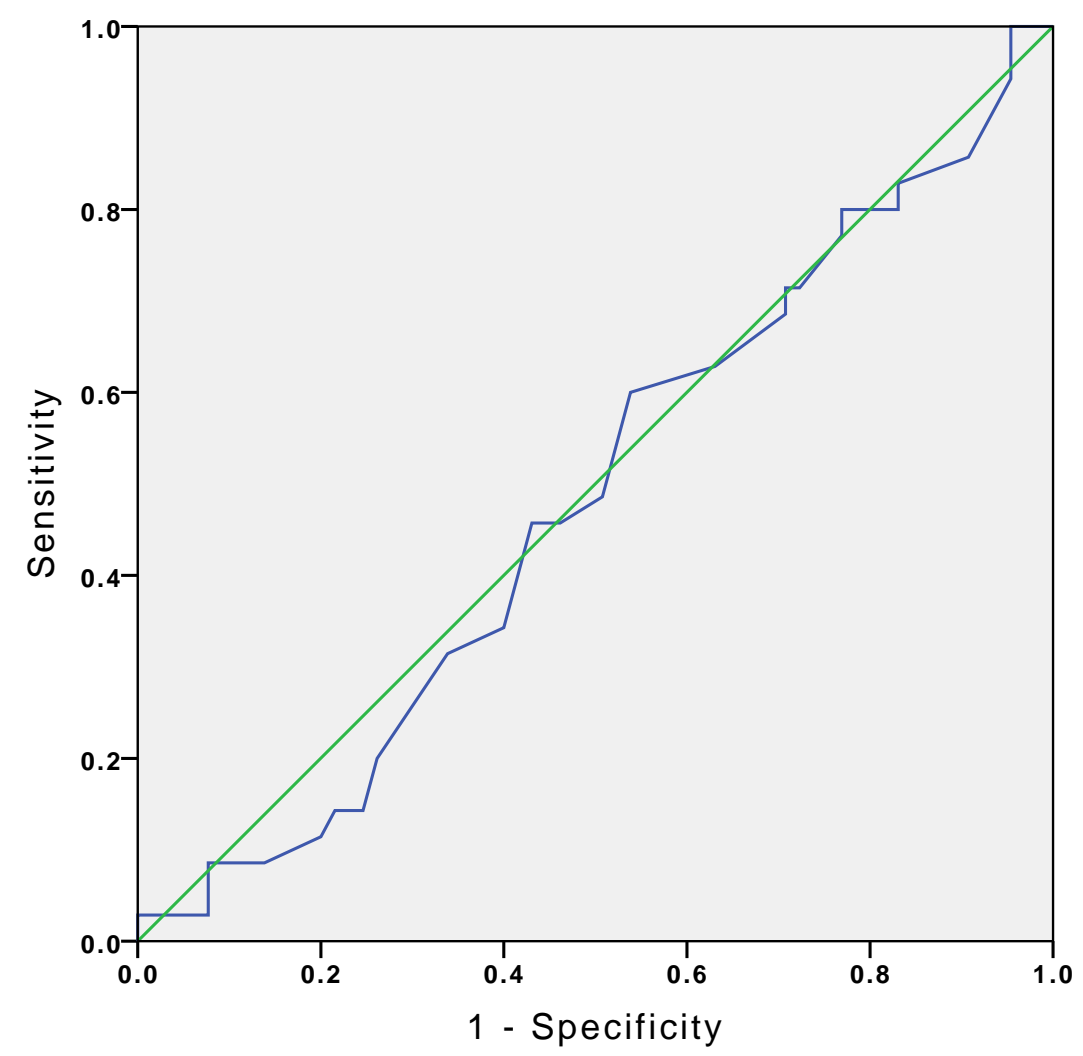

Figure 6-7 ROC curve analysis of residual platelet reactivity and MACE 
Major bleeding was also examined at 1 year and the only predictor of increased bleeding risk was AAA surgery $(8.5(2.9-24.8), p=0.001)$. LOWPR, elevated hs-TnT, elevated BNP and elevated CRP did not predict bleeding risk

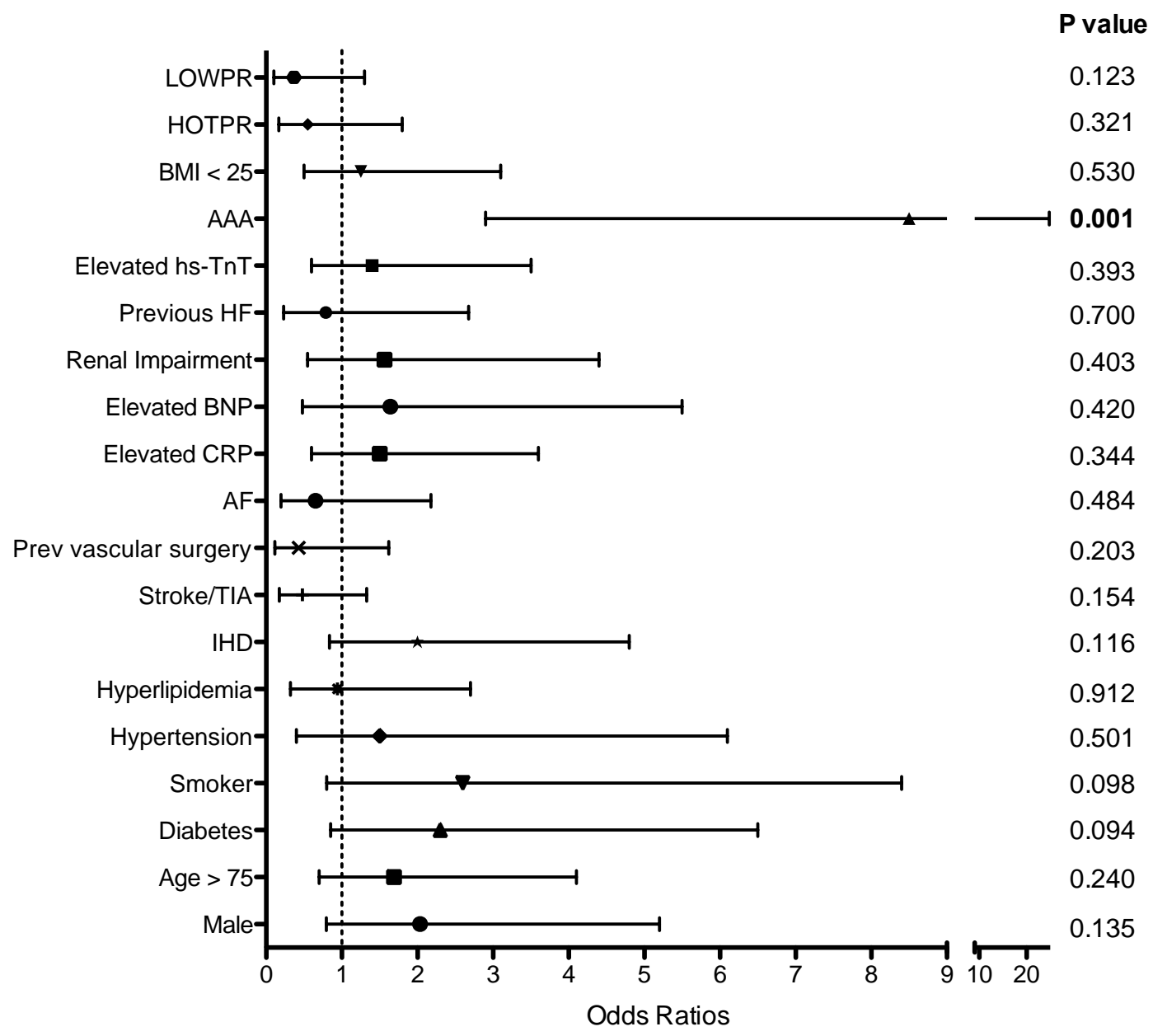

Figure 6-8 Predictors of bleeding at 1 year

Clinical and demographic variables represented as odds ratios for predictors of bleeding at 1 year and $95 \%$ confidence intervals.

ROC curve analysis was also conducted to investigate the relationship between residual platelet reactivity and bleeding at 1 year (Figure 6-9). The ROC curve displayed no relationship with an area under the curve 0.563 and no discernable cut-point that would identify patients at increased risk of bleeding. 


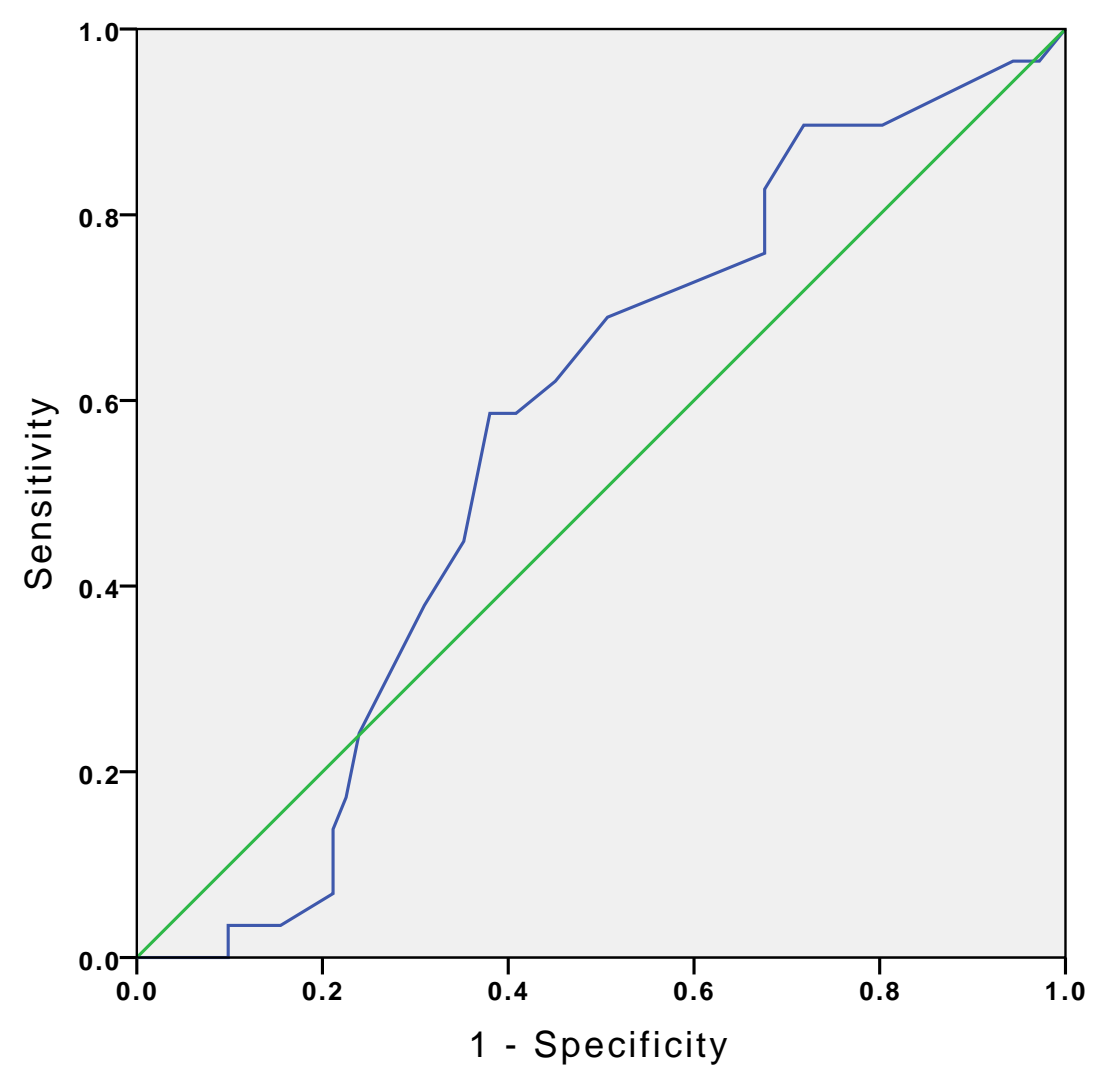

Figure 6-9 ROC curve analysis of residual platelet reactivity and bleeding

\subsubsection{Adverse outcomes short term}

Several clinical and demographic factors were identified to be predictive of MACE at 30 days in univariate analysis (Figure 6-10). These included a BMI <25 (4.3 (1.6-11.3), $p=0.002)$, elevated baseline hs-TnT $(2.5(1.0-6.7), p=0.05)$, and previous HF (2.8 $(0.96-8.7), p=0.05)$. All of these factors were found to be independent predictors of MACE at 30 days in multivariate analysis (Figure 6-11). HOTPR, elevated NT-proBNP and elevated CRP did not significantly correlate with short term MACE. 


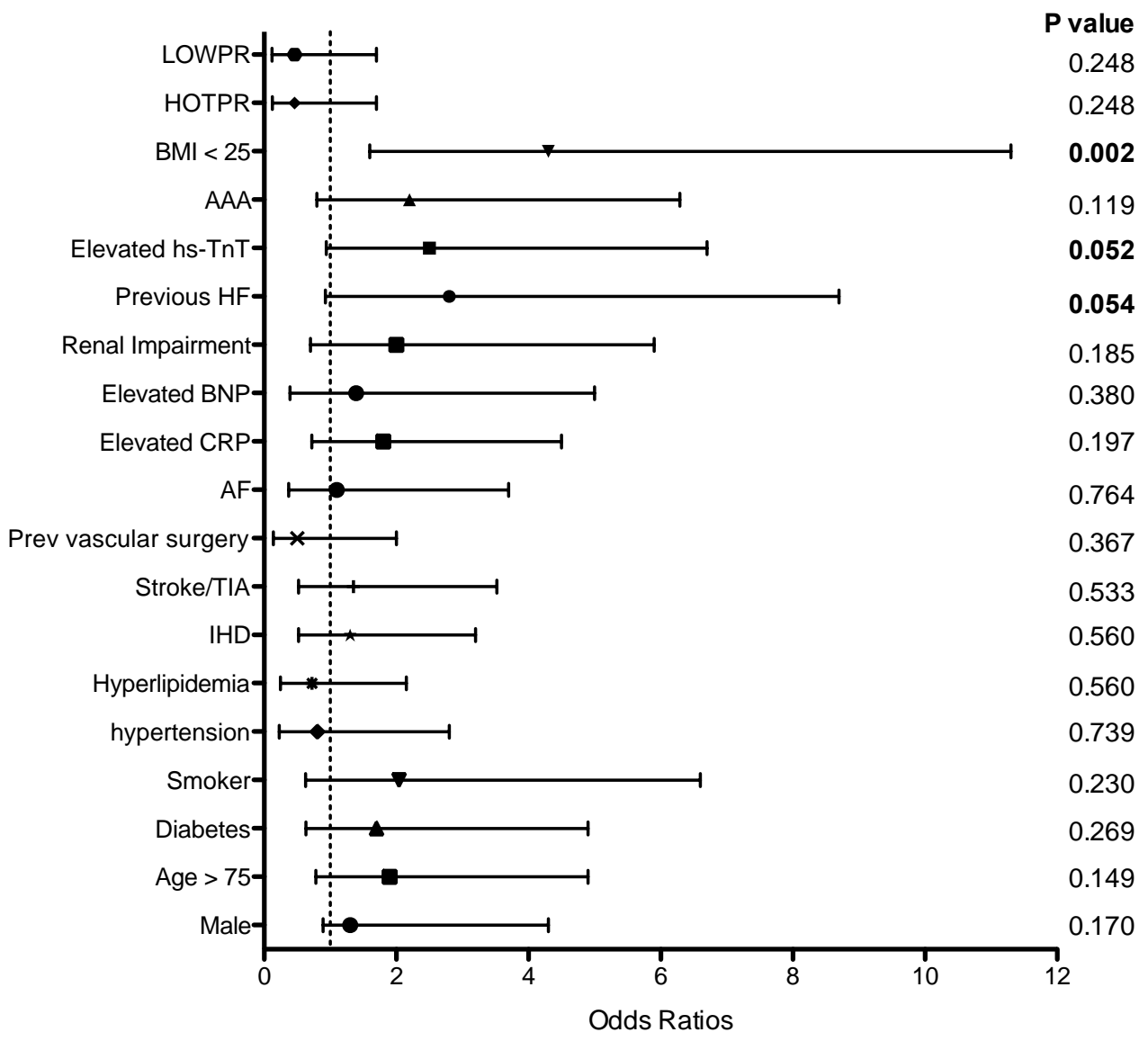

Figure 6-10 Predictors of short term MACE

Clinical and demographic variables represented as odds ratios for predictors of MACE at 30 days and $95 \%$ confidence intervals.

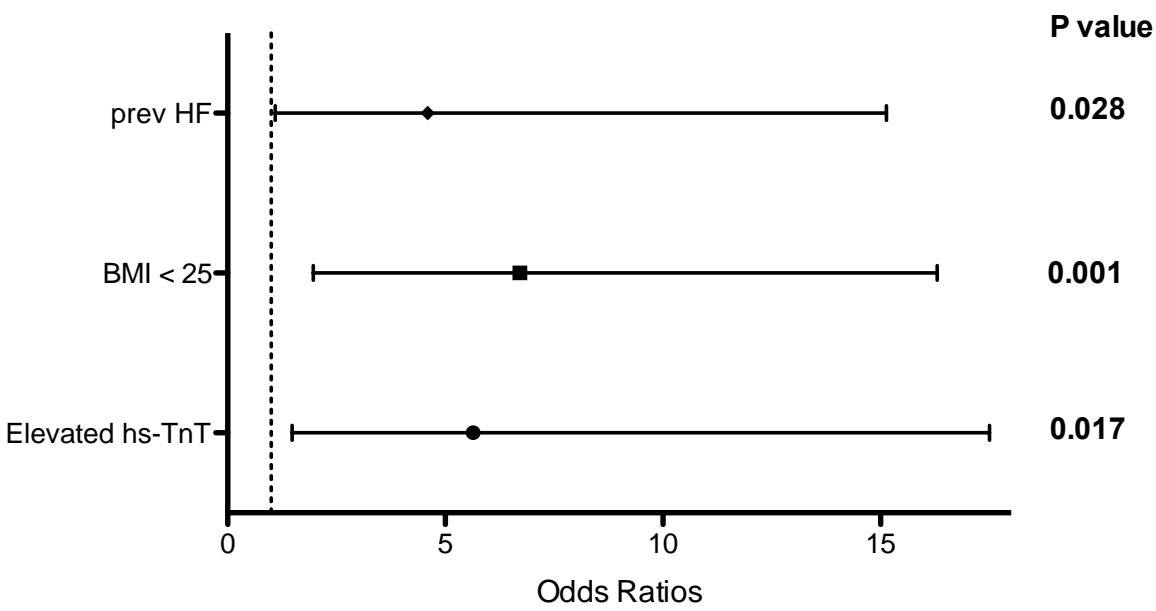

Figure 6-11 Multivariate analysis of short term MACE predictors

Clinical and demographic variables represented as odds ratios for predictors of MACE at 30 days and $95 \%$ confidence intervals. 
Short term bleeding was also examined and the only variable that was found to be significantly associated with an increased risk of bleeding was undergoing AAA surgery (Figure 6-12). Patients in this group had a 10 times higher risk of bleeding during their hospital admission (10.0 (3.3 - 29.7), $p=0.001)$. There was no correlation between baseline biomarkers and short term bleeding.

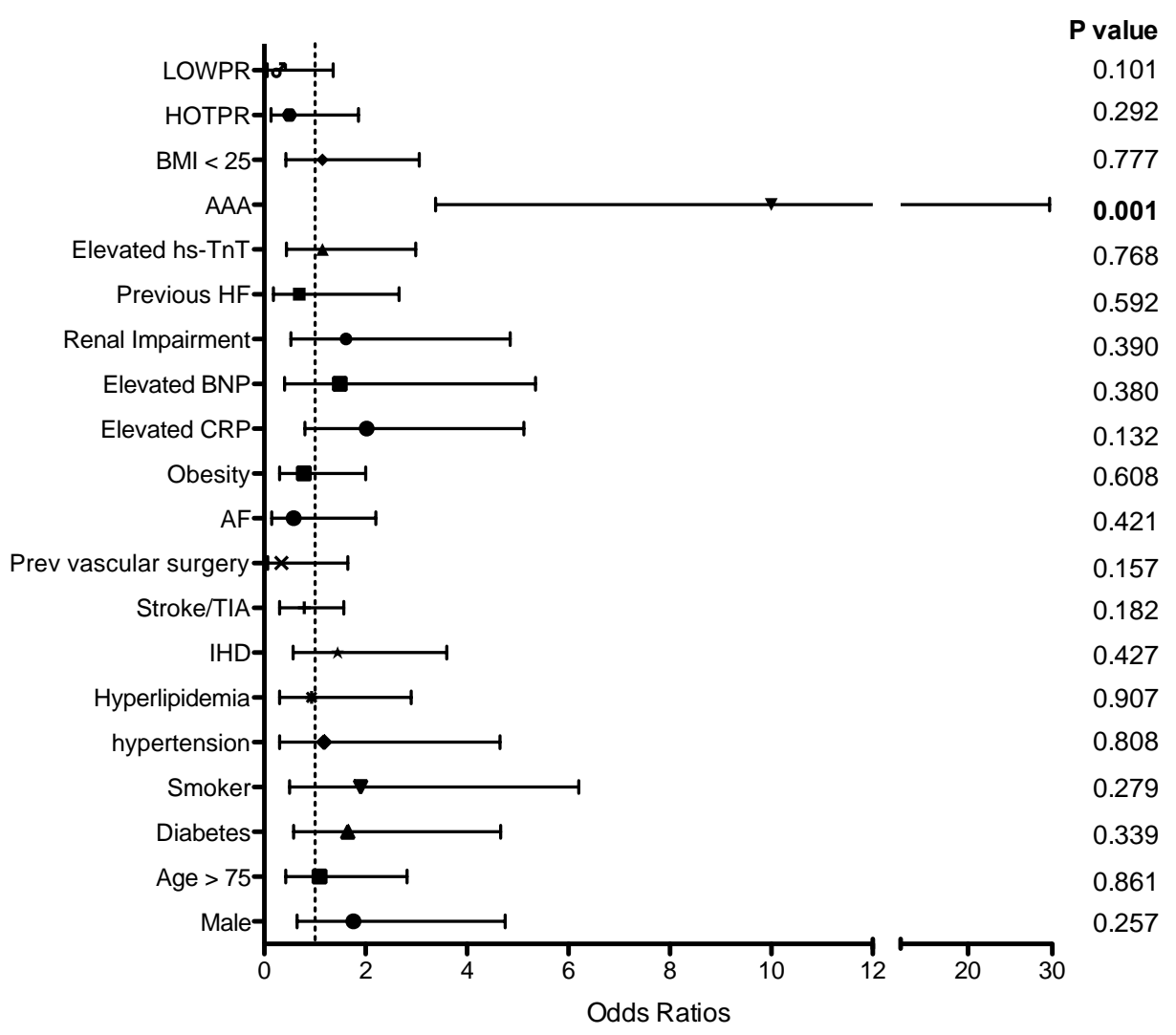

Figure 6-12 Predictors of short term bleeding

Clinical and demographic variables represented as odds ratios for predictors of bleeding at 30 days and $95 \%$ confidence intervals

Platelet reactivity was plotted for each bleeding and MACE from day 1 up to day 30 post surgery (Figure 6-13). This identified a group of patients that had co-localisation of both MACE and bleeding events. 


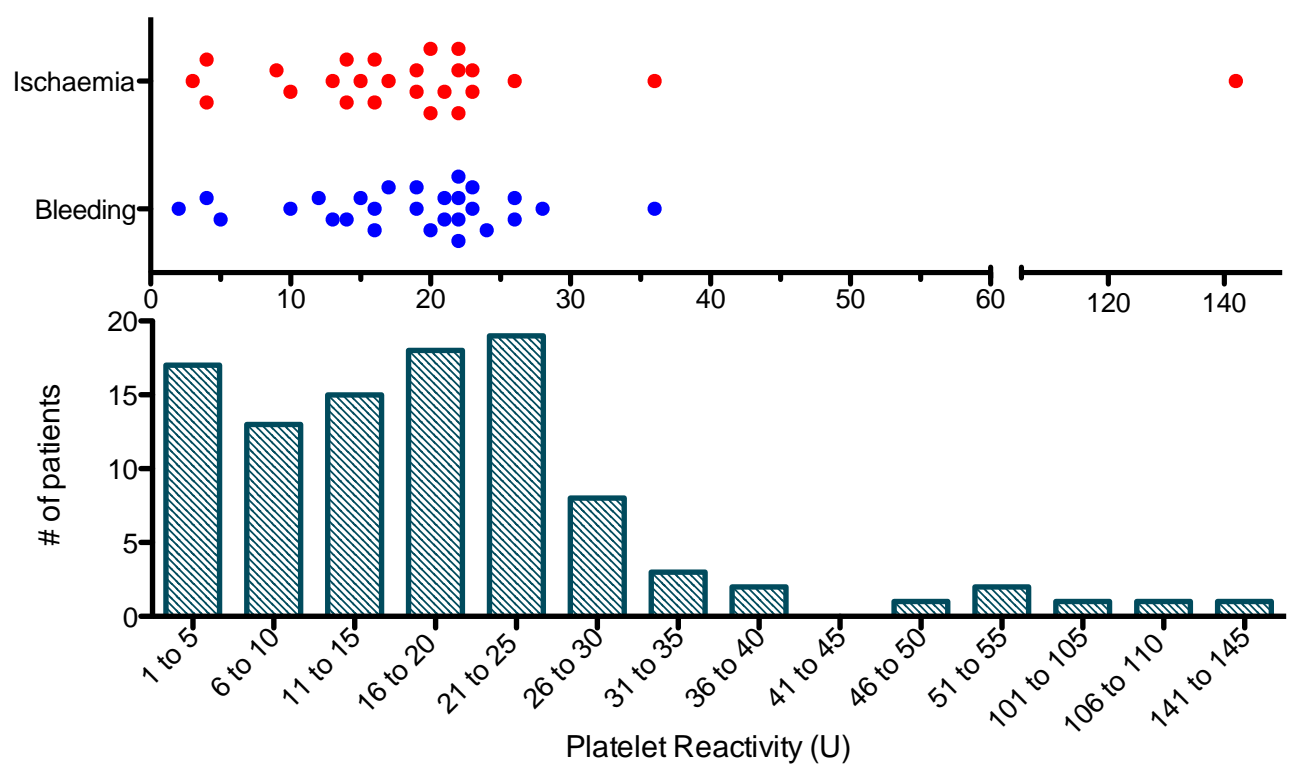

Figure 6-13 Platelet reactivity and short term events

The distribution of platelet reactivity $(U)$ compared to bleeding and MACE during the hospital admission. Each dot represents an individual MACE (red) or bleeding (blue) event in-hospital. Both $\mathrm{X}$ axis display platelet reactivity.

To assess whether clinical and demographic parameters could identify patients at risk of both major bleeding and MACE in this period, univariate analysis was conducted with the endpoint of bleeding plus MACE (Figure 6-14). In this analysis, patients undergoing AAA surgery (4.1 (1.2 - 13.9), $p=0.017)$ and patients with an elevated baseline hs-TnT (3.5 (1.1 - 11.8), p = 0.03) had an increased risk of experiencing both bleeding and MACE in the short term. An elevated baseline NT-proBNP (2.87 (0.989.52), $p=0.07$ ) resulted in a trend for more bleeding and MACE. Multivariate analysis identified AAA surgery (10.9 (2.0 - 59.5), p = 0.005) and elevated hs-TnT (6.9 (1.2 39.5), $p=0.02$ ) as independent predictors of the combination of bleeding and MACE in the time period up to 30 days post surgery (Figure 6-15). 


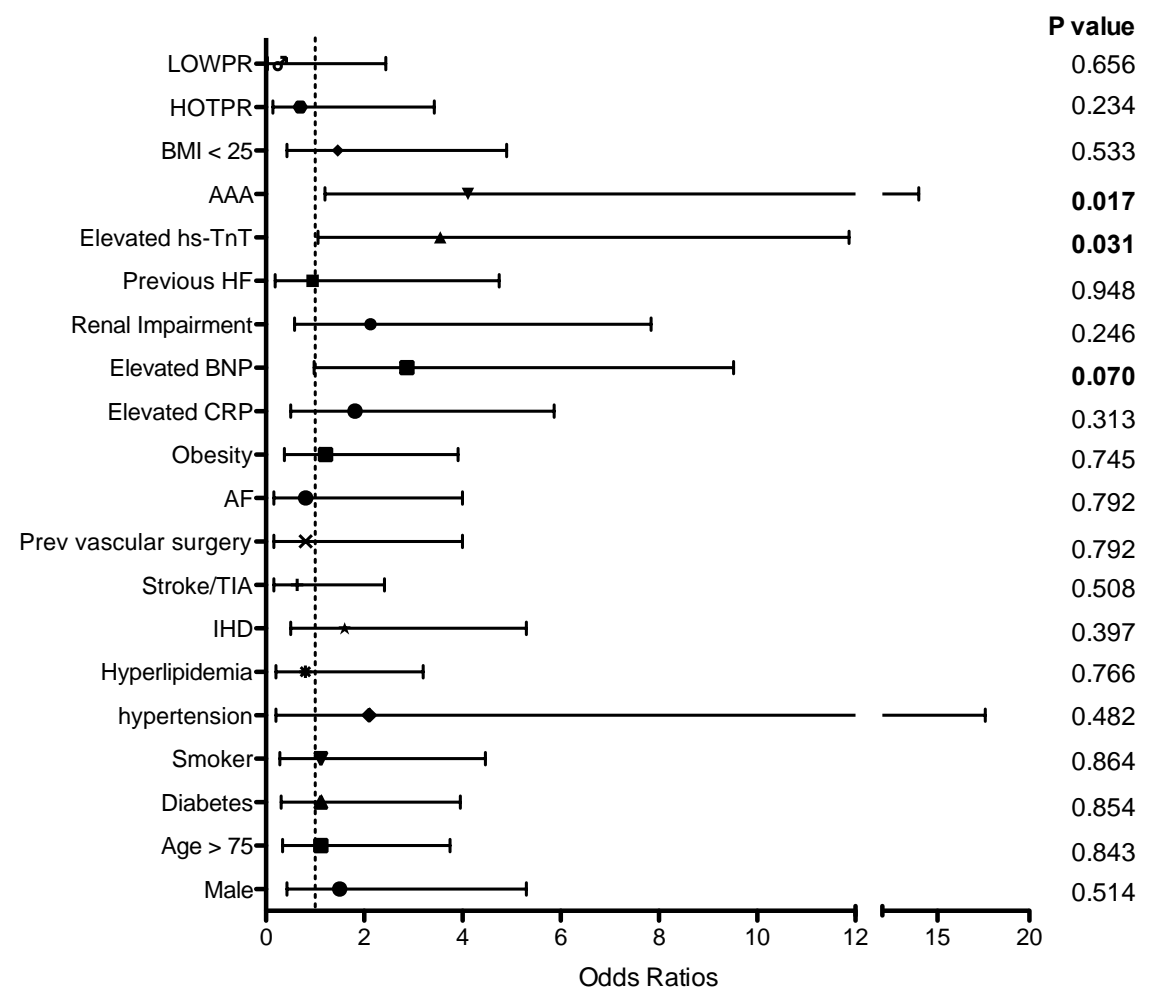

Figure 6-14 Predictors of patients at risk of both short term MACE and bleeding

Clinical and demographic variables represented as odds ratios for predictors of MACE and bleeding at 30 days and $95 \%$ confidence intervals

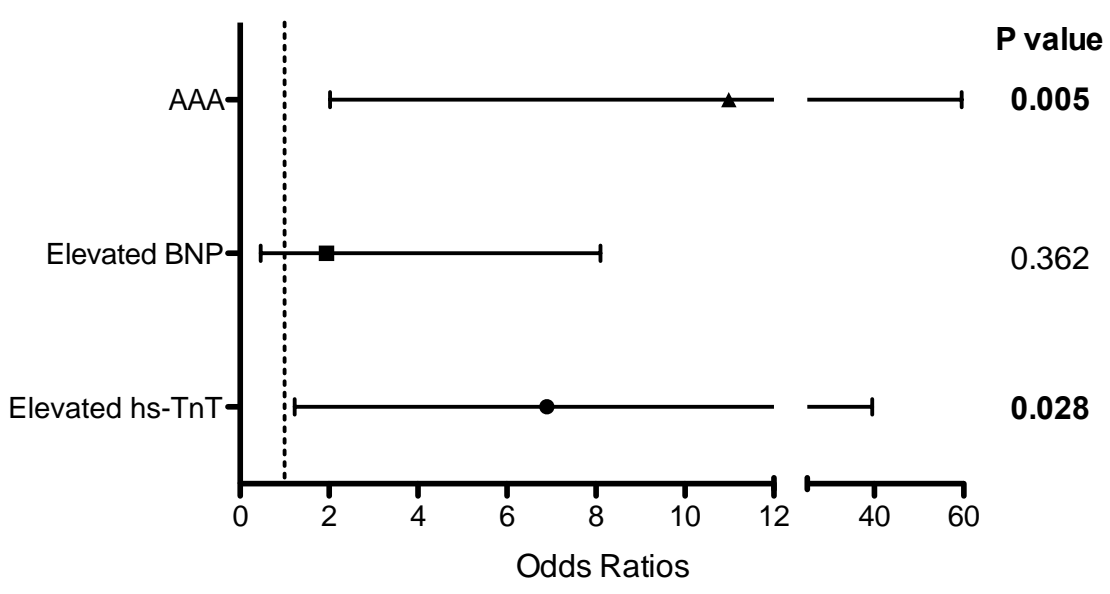

Figure 6-15 Multivariate analysis of predictors of patients at risk of short term MACE and bleeding

Clinical and demographic variables represented as odds ratios for predictors of MACE and bleeding at 30 days and $95 \%$ confidence intervals 


\subsubsection{Long term adverse outcomes}

Predictors of bleeding and MACE between 30 days and one year were also investigated. Only elevated hs-TnT $(4.1(1.2-13.6), \mathrm{p}=0.013)$ and elevated CRP $(3.3$ $(1.1-10.3), p=0.047)$ were associated with an increased risk of MACE during this time period (Figure 6-16). However, elevated hs-TnT was the only significant independent predictor in multivariate analysis resulting in a $3.7(1.1-12.3, p=0.034)$ increased risk of MACE between 30 days and 1 year (Figure 6-17).

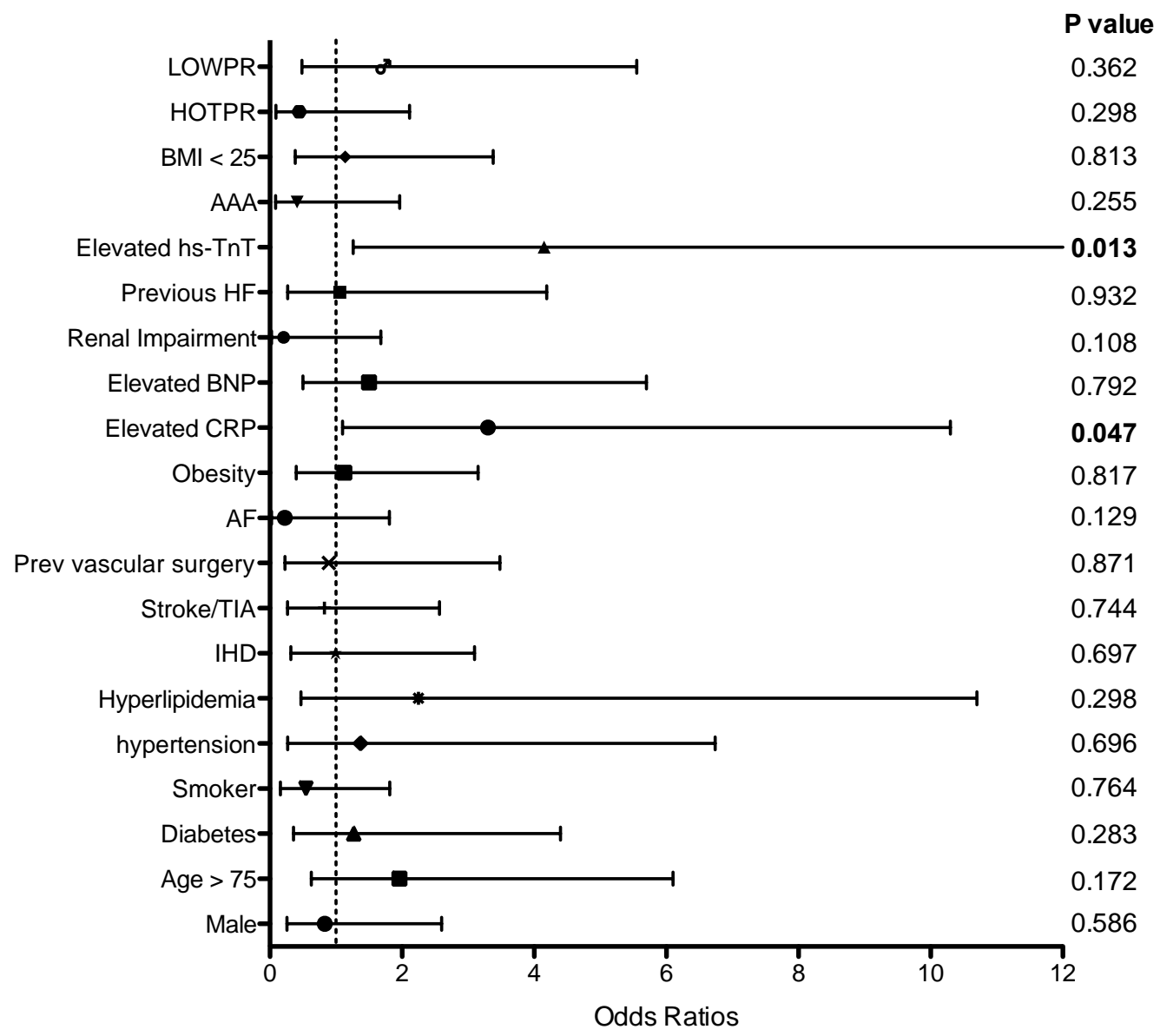

Figure 6-16 Predictors of long term MACE

Clinical and demographic variables represented as odds ratios for predictors of MACE between 30 days and 1 year and $95 \%$ confidence intervals 


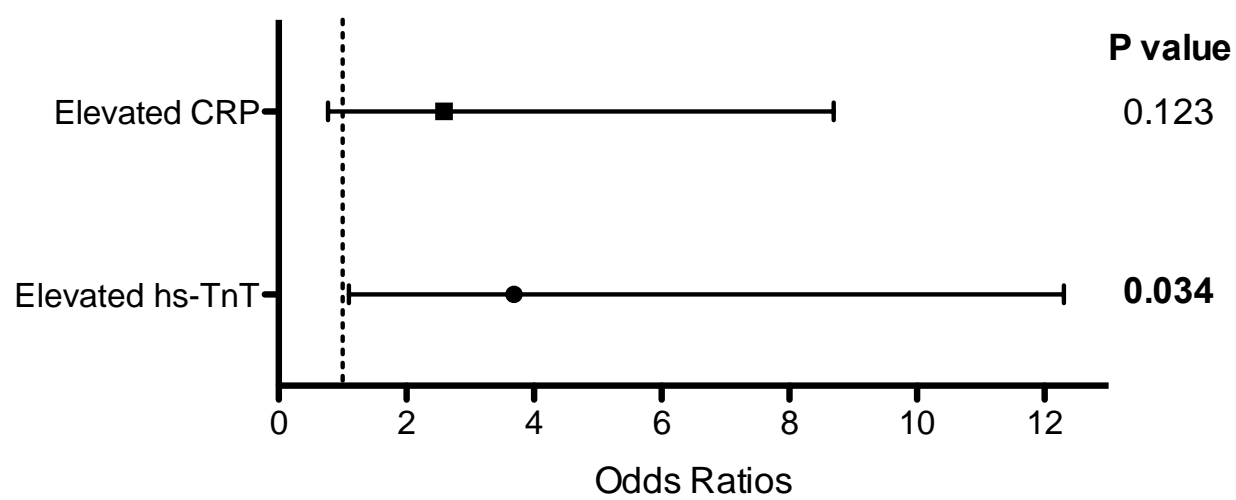

Figure 6-17 Multivariate analysis of predictors of long term MACE

Clinical and demographic variables represented as odds ratios for predictors of MACE between 30 days and 1 year and $95 \%$ confidence intervals

There were no baseline clinical factors or biomarker that significantly predicted an increased risk of long term bleeding (Figure 6-18).

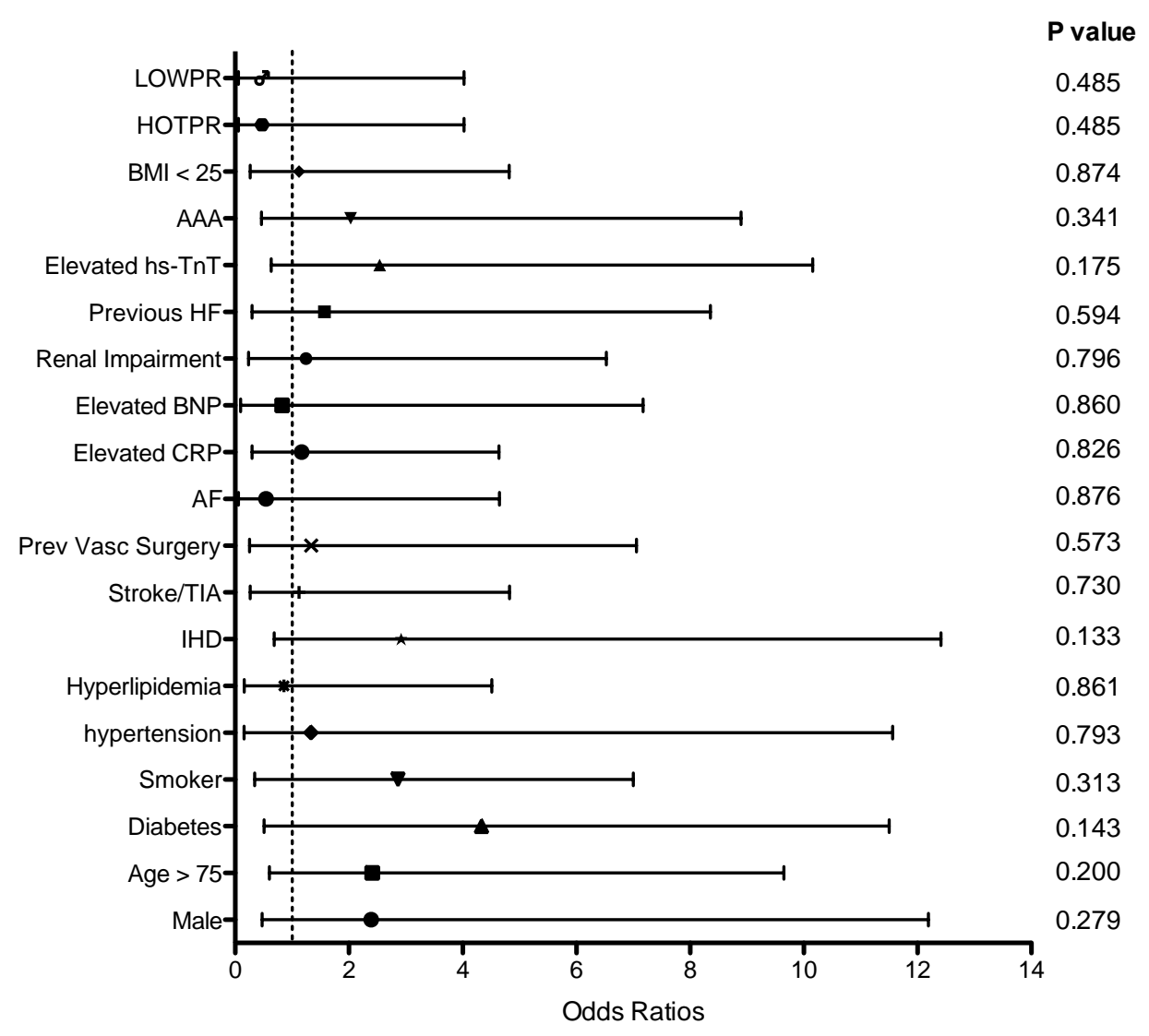

Figure 6-18 Predictors of long term bleeding

Clinical and demographic variables represented as odds ratios for predictors of bleeding between 30 days and 1 year and 95\% confidence intervals 
Platelet reactivity was plotted for each bleeding and MACE event from 30 days up to 1-year post surgery (Figure 6-19). In contrast to short-term events, there was no colocalisation of MACE and bleeding in this time period. Furthermore, platelet reactivity levels were not significantly different in patients experiencing MACE compared to those having major bleeds.

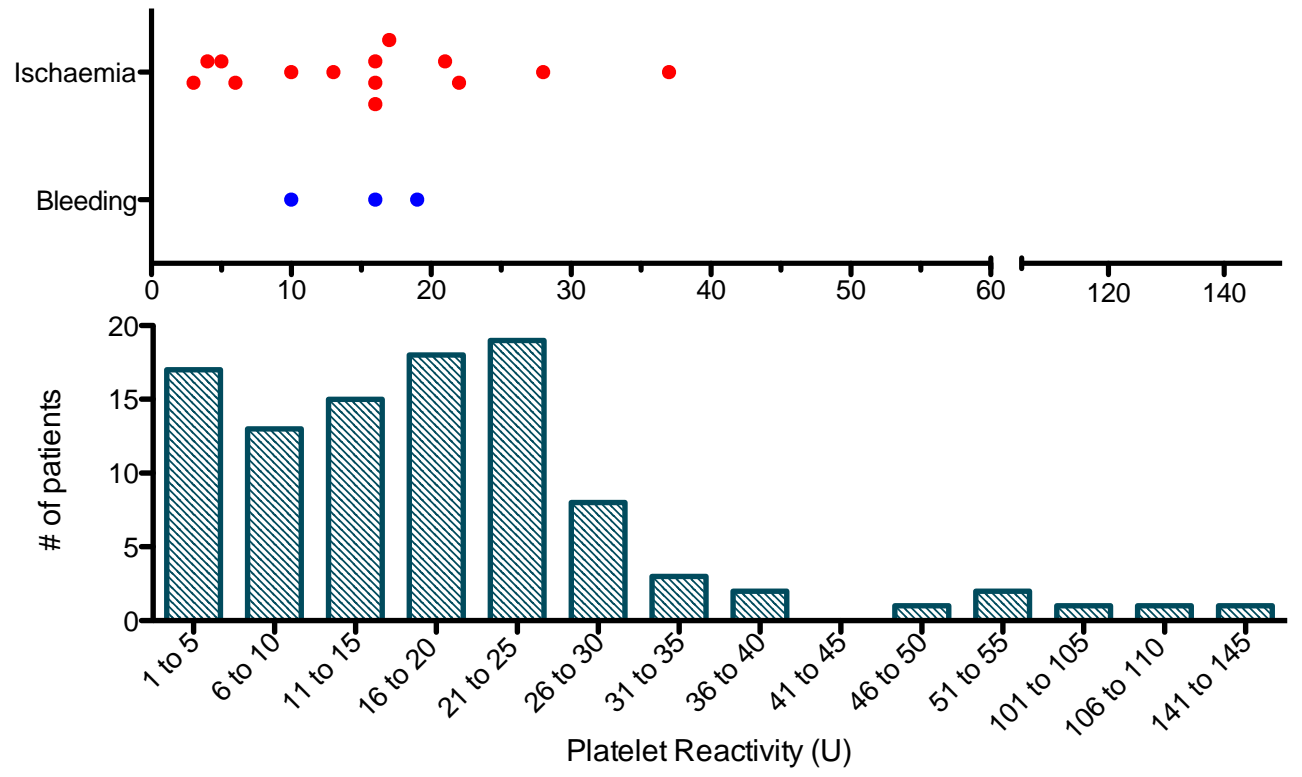

Figure 6-19 Platelet reactivity and long term events

The distribution of platelet reactivity (AU) compared to bleeding and MACE from 30 days up to 1 year post surgery. Each dote represents an individual MACE (red) or bleeding (blue) event at 1 year. Both $\mathrm{X}$ axis display platelet reactivity. 


\subsection{Discussion}

This is the first study to investigate the relationship between residual platelet reactivity and other baseline cardiac biomarkers with outcomes following elective major vascular surgery. Residual platelet reactivity both high and low was not found to be significantly associated with MACE or bleeding following surgery in this cohort. Elevated baseline hs-TnT appeared to be the most predictive biomarker and was significantly associated with the risk of MACE in both the short-term and long-term. Bleeding occurred early following surgery in the majority of patients and AAA surgery was the only predictor of bleeding. Interestingly, we found a number of MACE and bleeding events were co-located, which may indicate that mechanisms other than a thrombotic process could be driving ischaemic events in these patients.

We found the response to aspirin in our vascular cohort to be highly variable ranging between $2 \mathrm{AU}$ to $142 \mathrm{AU}$. The level of on treatment residual platelet reactivity did not correlate with adverse outcomes in this vascular cohort and we could not derive a cutpoint for HOTPR from ROC curve analysis. Using the previously recommended cut point for aspirin HOTPR of $30 \mathrm{AU}$, which has been derived in a healthy population (295), only $10 \%$ of patients in our study would be classified as having a suboptimal response to aspirin. Our findings are consistent with a previous report, which estimated that high on treatment platelet reactivity was present in between $5 \%$ and $27.5 \%$ of patients with PVD depending on the assay used (371).

To date the few studies that have examined the relationship between residual platelet reactivity and adverse events in those undergoing vascular surgery have demonstrated mixed findings. Rajagopalan and colleagues assessed platelet function with the VerifyNow arachidonic acid assay in 136 aspirin treated patients undergoing elective surgery for subcritical limb ischemia or infrarenal AAA repair. They found that postoperative but not baseline arachidonic acid stimulated platelet aggregation was higher in those with a postoperative troponin rise (153). Another study of patients on aspirin therapy undergoing elective vascular surgery found that when platelet function was assessed using whole blood impedance aggregometry with arachidonic 
acid stimulation those with residual platelet reactivity in the upper quartile had almost twice the rate of in-hospital cardiovascular events (154). Finally, a study conducted in patients with peripheral arterial vascular disease undergoing transluminal angioplasty found a high level of residual platelet reactivity on aspirin as measured by light transmission aggregometry, did not correlate with the rate of stent thrombosis/restenosis (372).

There are a number of possible explanations as to why residual platelet reactivity didn't correlate with ischaemic outcomes in our study. It is likely that a significant proportion of perioperative myocardial infarctions are due to an imbalance between myocardial oxygen supply and demand (type $2 \mathrm{MI}$ ). Multiple factors including high circulating catecholamine levels, vasospasm, endothelial dysfunction, hypotension and hypoxia in the perioperative period have the potential to cause a type $2 \mathrm{MI}(375)$. Consistent with this concept a recent study demonstrated that coronary plaque rupture was present in slightly less than half of those suffering periprocedural MI following non-cardiac surgery (374). Whilst platelet activation plays a central role in type $1 \mathrm{MI}$, it may not play the same role in perioperative type $2 \mathrm{MI}$ and as a result may not be predictive of these events.

Another potential explanation for the failure of residual platelet reactivity to predict ischaemic events is that we studied a population with stable PVD. Although we found the response to aspirin to be highly variable, only $10 \%$ of patients had platelet reactivity values $>30 \mathrm{AU}$ or high on treatment platelet reactivity. Studies in patients presenting with ACS have clearly shown high on treatment platelet reactivity to be predictive of future ischaemic events (375). However, studies of platelet function in those with stable coronary artery disease been less convincing and have failed to demonstrate an incremental predictive value over common risk factors for the occurrence of major adverse cardiovascular events $(375,376)$. Previous studies have shown patients presenting with critical limb ischaemia to have very high levels of platelet activation (377). Therefore it is possible that a poor response to aspirin may occur more frequently in a population presenting acutely for vascular surgery and may be more predictive of outcome. 
The introduction of high-sensitivity cardiac troponin assays, which have a 10 times greater sensitivity compared with previous assays, has allowed the accurate determination of very low levels of circulating troponin $(378,379)$. As a result the elderly, and those with other comorbidities such as renal impairment, stable coronary disease and heart failure frequently have detectable circulating troponin, which may be elevated above the upper limit of normal (382). It is therefore not surprising $35 \%$ of our cohort had elevation of baseline hs-TnT above the upper limit or normal. Measurement of cardiac troponin has become the cornerstone for the diagnosis of myocardial infarction. Because of the possibility of background chronic elevations a rising or falling pattern of troponin levels is key to the diagnosis of MI. In our study there were 28 patients who had an elevated baseline hs-TnT that remained stable and elevated post operatively that potentially could have been misclassified as having a periprocedural event if post operative hs-TnT alone had been measured.

Baseline hs-TnT was the biomarker most predictive of ischaemic events in our study. An elevated baseline hs-TnT was independently predictive of MACE at both 30 days and 1 year. Other studies have reported that an elevated post-operative troponin or periprocedural enzyme rise is predictive of long term outcomes $(366,367)$. To our knowledge this is the first study to demonstrate the utility of preoperative high sensitivity troponin in predicting risk of cardiovascular events in patients undergoing vascular or non-cardiac surgery. Elevated baseline hs-TnT has consistently been demonstrated to be a strong predictor of poor cardiovascular outcomes in a range of other patient populations. These include but are not limited to; patients with acute (383) and chronic coronary disease (382), in patients with congestive heart failure (383), in hemodialysis patients (386) and even healthy older patients (385). Our findings suggest that further investigation is warranted to confirmed the uitility of preoperative hs-TnT for risk stratification and to determine whether this is additive to existing risk stratification models.

Elevated baseline CRP was associated with a higher risk of MACE at 1 year in univariate analysis but was not found to be a significant predictor in multivariate analysis. CRP is 
an acute-phase protein and appears in the circulation in response to inflammatory cytokines, such as IL-6 and can act as a biomarker for systemic inflammation (386). Elevated levels of CRP in patients experiencing an ACS have shown to be a predictor of poor cardiovascular outcomes both short and long term $(387,388)$. The biomarker has also found to predictive of mortality in end-stage renal disease patients, independent of other classic biomarkers (389). Elevated CRP as a predictor of longterm MACE in vascular patients, may reflect a higher level of background inflammation and indicate a more unstable atherosclerotic process, as seen in CAD populations (392).

NT-proBNP is one of a family of cardiac peptides secreted almost exclusively by the myocardium in a nonspecific response to wall stress (391). Several studies have identified strong associations between elevated preoperative levels of NT-proBNP with adverse cardiac events both perioperatively and long term following non-cardiac surgery (392-394). Our study found a weak relationship between elevated baseline NT-proBNP and the occurrence of both bleeding and MACE in-hospital. Due to reduced power and large confidence intervals around the elevated risk, this association was not significant. However, previous HF was found to be a significant predictor of short-term MACE. A history of HF has also been identified as a cardiac risk factor for patients undergoing non-cardiac major surgery and proposed as an important component to be incorporated in to preoperative risk scores (397). It remains unclear how much additive prognostic value NT-proBNP levels can offer to stratify risk in vascular surgery patients.

The other clinical factors that were found to be independently associated with increased MACE risk were a BMI $<25$ and AAA surgery. A lower BMI has previously been associated with a higher risk of death following major cardiac surgery compared to normal weight or obese patients (396). However, this may also be a reflection of a patients' frailty, which has also been linked to poor outcome and has increasing interest as an important factor to integrate into pre-operative risk scores for cardiac surgery (397). Open AAA surgery was associated with an increased risk of short term MACE and co-located with bleeding. This finding is not surprising as it is well 
established that AAA surgery is the type of vascular surgery associated with the highest risk of ischaemic events (398). Interestingly, AAA surgery was not associated with long-term MACE risk in our cohort. This is consistent with the large randomised trials investigating open AAA repair versus endovascular approaches, which found that the risk of open AAA occurs predominantly in the perioperative setting with a large decline in adverse events past 30 days (399).

Major bleeding was found to be common with the vast majority of events occurring within the first 30 days. The AAA surgery group had 10 times higher risk of major bleeding and was the only significant factor identified. Several previous trials of therapy for AAA patients have also observed high rates of major bleeding. In the Dutch Randomized Endovascular Aneurysm Management (DREAM) trial, 39\% of the 345 patients submitted to aortic aneurysm repair received blood transfusions (398). Furthermore, in the Open Versus Endovascular Repair (OVER) trial, the authors observed a median of $1000 \mathrm{~mL}$ blood loss during surgery in the 429 open AAA patients enrolled (399). This is similar to the observed $1045 \mathrm{~mL}$ average blood loss in our AAA surgery patient group. 


\subsection{Limitations}

Firstly, in order to have the power to detect a relationship between biomarkers and patient outcome, the study was designed to recruit 240 patients undergoing major vascular surgery. Although this power calculation was based on a predicted adverse cardiovascular outcome rate of $21-24 \%$ and we experienced a much higher rate at $34 \%$, a cohort size of 100 patients leaves the study underpowered. When the inclusion/exclusion criteria were being determined, we did not fully appreciate the way in which the vascular service operated, particularly how they managed surgery waiting times and operation theatre scheduling. A management scheme which appears to be somewhat unique to the Wellington service, resulted in a large proportion of planned elective cases being pushed through on a semi-acute theatre list to get around limited vascular theatre access and long waiting lists. This resulted in a significantly reduced pool of eligible patients to recruit from. With a larger patient group we may have been able to detect significant relationships between some of the biomarkers and other clinical factors with cardiovascular and bleeding outcomes. The heterogeneity of the vascular procedures in the study could be considered a limitation. However, many previous studies have recruited similar population groups (367-369). All patients have atherosclerotic vascular disease and underwent major surgery. Our study population is somewhat restrained compared to other studies that have included a mix of those undergoing vascular surgery with endovascular interventions $(154,400)$ and elective and acute patients which arguably results in more variance in levels of perioperative risk between patients. 


\subsection{Conclusion}

Patients undergoing major vascular surgery remain at a high risk of experiencing perioperative and long-term adverse events. We have demonstrated for the first time that elevated hs-TnT at baseline is an independent risk factor for short and long term MACE in patients undergoing vascular surgery. Our findings suggest further investigation of the utility of baseline hs-TnT for pre-operative risk stratification is warranted. Although an elevated CRP was predictive of MACE at one year on univariate analysis, in multivariate analysis it was no longer predictive. Residual platelet reactivity on aspirin was not associated with either MACE or bleeding outcomes in our cohort. Major bleeding was frequent with the vast majority occurring early. This observation and the observation that bleeding and MACE frequently occurred concurrently suggests that the use of more potent antiplatelet therapy in the perioperative period in an attempt to reduce adverse events may not be a successful strategy. The majority of bleeding occurred in-hospital and AAA surgery was the only significant determinant of a major bleed occurring. This study supports further investigation of the incorporation of biomarkers, particularly hs-TnT into risk models that will enable the identification of patients that will benefit from additional preoperative evaluation. 
CHAPTER 6

164 
7 Chapter 7: Platelet-T cell interactions 


\subsection{Introduction}

Historically, platelets have been noted for their significant thrombotic role in atherosclerotic plaque rupture. The previous chapters have focused on residual platelet reactivity as a driver or primarily thrombotic risk in patients with coronary artery disease and vascular disease. More recently, platelets have been established as cells that directly contribute to plaque progression (as reviewed in Section 1.3.2), which may also contribute to risk. This involvement has been demonstrated in several atherosclerotic mouse and in vitro human development models, revealing platelets bind to the endothelium and leukocytes, primarily through P-selectin, resulting in enhanced leukocyte recruitment and migration into the plaque (75). Additionally the high expression of CD40L (CD154) on platelet membranes and the release of soluble CD40L contribute significantly to the pro-inflammatory milieu of atherosclerotic plaques (94).

Platelet-monocyte interactions have been thoroughly investigated in atherosclerosis including ACS. Platelet-monocyte conjugates have been shown to be increased in ACS patients, with platelet interactions inducing a more pro-inflammatory phenotype in circulating monocytes (403). High levels of the CD14++CD16+ monocyte subtypeplatelet conjugates has been correlated with a higher troponin release ACS and is predictive of poor outcome as indicated by a decreased left ventricular ejection fraction (404). Interestingly, loading ACS patients with clopidogrel decreased plateletmonocyte conjugates, monocyte activation and the plasma levels of pro-inflammatory mediators RANTES and CD4OL (405); further adding to the hypothesis that platelets drive pro-inflammatory environments.

The interactions between platelets and lymphocytes are less well understood. It is confirmed that platelets and lymphocytes can bind and form conjugates which are preferentially formed with T cells. This connection is established via platelet P-selectin and lymphocyte P-selectin glycoprotein ligand-1 (PSGL-1) leading to enhanced migration of lymphocytes to plaques (406). In contrast, CD40-CD40L cross-linking enhances $\mathrm{T}$ cell cytokine production (407). Platelets also release several soluble 
factors that may influence $T$ cell activation, including platelet factor 4 (PF4), transforming growth factor- $\beta$ (TGF- $\beta$ ) and RANTES. However work done to date has been conducted in predominantly mouse models of disease or in healthy individuals and may not be relevant to how platelets function in patients with atherosclerosis. Therefore the focus of this chapter was to investigate differences in platelet-T cell interactions in patients with atherosclerotic vascular disease compared to healthy controls.

The specific aims of this study were:

1. To determine the level of platelet-T cell conjugates in healthy and vascular patients.

2. To investigate differences in platelet conjugates with $\mathrm{T}$ cell subgroups (CD4, CD8 and natural killer T cells) between the two patient groups.

3. To assess alterations in the activation phenotype of CD4 and CD8 T cells between healthy and disease state and any changes in platelet interactions with these cells.

\subsection{Methods}

\subsubsection{Study population}

Two patient groups were included to investigate platelet - $\mathrm{T}$ cell conjugates. All patients provided written informed consent and the study was reviewed and approved by the Upper South A Regional Ethics Committee (URA/11/05/016).

\subsubsection{Healthy volunteers}

Participants enrolled in the study were between the ages of $18-50$ years with no known cardiovascular disease, or other acute illness within the preceding six weeks. Exclusion criteria included diabetes, smokers, pregnancy, intercurrent illness and those who were on regular cardiovascular or immune modulating medication or had been treated with antiplatelet therapies or non-steroidal anti-inflammatory drugs (NSAID) within the last 7 days. 


\subsubsection{Vascular patients}

Patients in whom the presence of peripheral atherosclerotic vascular disease had been confirmed angiographically were recruited into the vascular cohort. Exclusion criteria included treatment with an antiplatelet agent or NSAID within 7 days of enrolment, a platelet count less than $100 \times 10^{9} / \mathrm{L}$, a known platelet function or bleeding disorder or intercurrent illness.

\subsubsection{Blood collection and antibody staining}

Blood for platelet-lymphocyte conjugate analysis was collected into a single hirudin tube as previously described (section 2.2.2). Whole blood was stained in duplicate with the four antibody panels made up in FACS buffer (Table 7-1) at 30 minutes post blood sampling. Appropriate isotype controls were stained alongside panel samples (Appendix 2). A total staining volume of $50 \mu \mathrm{L}$ (1:1 dilution of blood to antibody mix) was incubated in $1.5 \mathrm{~mL}$ eppendorfs for 20 minutes at room temperature (RT) in the dark. Following staining, $450 \mu \mathrm{L}$ of FACS Lyse containing 1\% paraformaldehyde (PFA) (BD Biosciences, NJ, USA) was added and gently mixed to lyse red blood cells and fix the sample for acquisition.

\subsubsection{Flow cytometry}

Flow cytometry was used to identify $T$ cell and platelet populations and acquired using the Millipore Guava easyCyte 8HT system (Merck Millipore, Darmstadt, Germany). Samples were diluted with FACS buffer before acquisition to achieve an optimal cell concentration between 300-500 cells/ $\mu \mathrm{L}$. Anti-mouse Ig, $\mathrm{k} /$ compensation beads (BD Biosciences, NJ, USA) were acquired the same time as samples for compensation during analysis. Data was analysed using FlowJo software (Tree star, Ashland, OR, USA).

At least $5000 \mathrm{~T}$ cell events were collected in every sample by gating on the CD3 positive cells. Platelet-Tcell conjugates with the different T cell subgroups were determined by the gating strategies outlined in Figures 7-1, 7-2 and 7-3. 


\section{CHAPTER 7}

Table 7-1 Platelet-T cell conjugates antibody panels

\begin{tabular}{|c|c|c|c|c|c|c|c|c|}
\hline & Panel 1 & & Panel 2 & & Panel 3 & & Panel 4 & \\
\hline Channel & Antibody & Final Conc & Antibody & Final Conc & Antibody & Final Conc & Antibody & Final Conc \\
\hline FITC & CD3 & $500 \mathrm{ng} / \mathrm{mL}$ & CD3 & $500 \mathrm{ng} / \mathrm{mL}$ & CD3 & $500 \mathrm{ng} / \mathrm{mL}$ & CD3 & $500 \mathrm{ng} / \mathrm{mL}$ \\
\hline $\mathrm{PE}$ & CD8 & $15 \mathrm{ng} / \mathrm{mL}$ & CD44 & $1 \mu \mathrm{g} / \mathrm{mL}$ & CD44 & $1 \mu \mathrm{g} / \mathrm{mL}$ & CD56 & $250 \mathrm{ng} / \mathrm{mL}$ \\
\hline PerCP & CD42a & $1.25 \mu \mathrm{g} / \mathrm{mL}$ & CD42a & $1.25 \mu \mathrm{g} / \mathrm{mL}$ & CD42a & $1.25 \mu \mathrm{g} / \mathrm{mL}$ & CD42a & $1.25 \mu \mathrm{g} / \mathrm{mL}$ \\
\hline PE-Cy7 & CD4 & $500 \mathrm{ng} / \mathrm{mL}$ & CD4 & $500 \mathrm{ng} / \mathrm{mL}$ & CD8 & $250 \mathrm{ng} / \mathrm{mL}$ & CD4 & $500 \mathrm{ng} / \mathrm{mL}$ \\
\hline APC & CD25 & $250 \mathrm{ng} / \mathrm{mL}$ & CD62L & $500 \mathrm{ng} / \mathrm{mL}$ & CD62L & $500 \mathrm{ng} / \mathrm{mL}$ & CD25 & $250 \mathrm{ng} / \mathrm{mL}$ \\
\hline
\end{tabular}

* A list of all antibody formats, clones, isotypes and manufactures can be found in Appendix 2. 

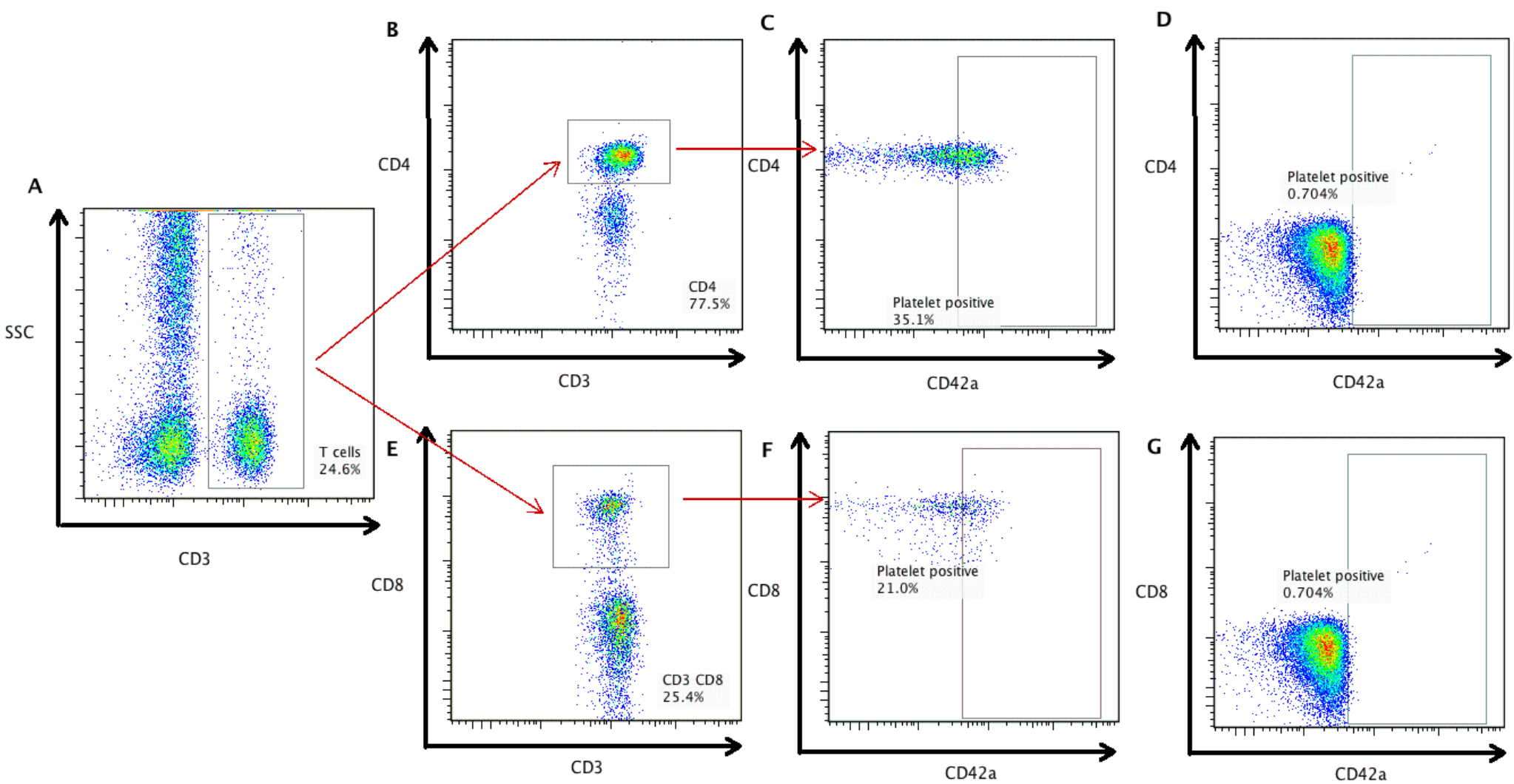

Figure 7-1 Gating strategies to identify CD8 and CD4 platelet-T cell conjugates.

T cells were identified by their SSC properties and expression of CD3 (A). CD4 and CD8 T cells were then identified by their expression of CD4 (B) and CD8 (E) respectively. Gating on these separate T cell population, CD42a could be used to identify platelet positive populations (C \& F) as compared to the isotype control (D \& G). 


\section{CHAPTER 7}

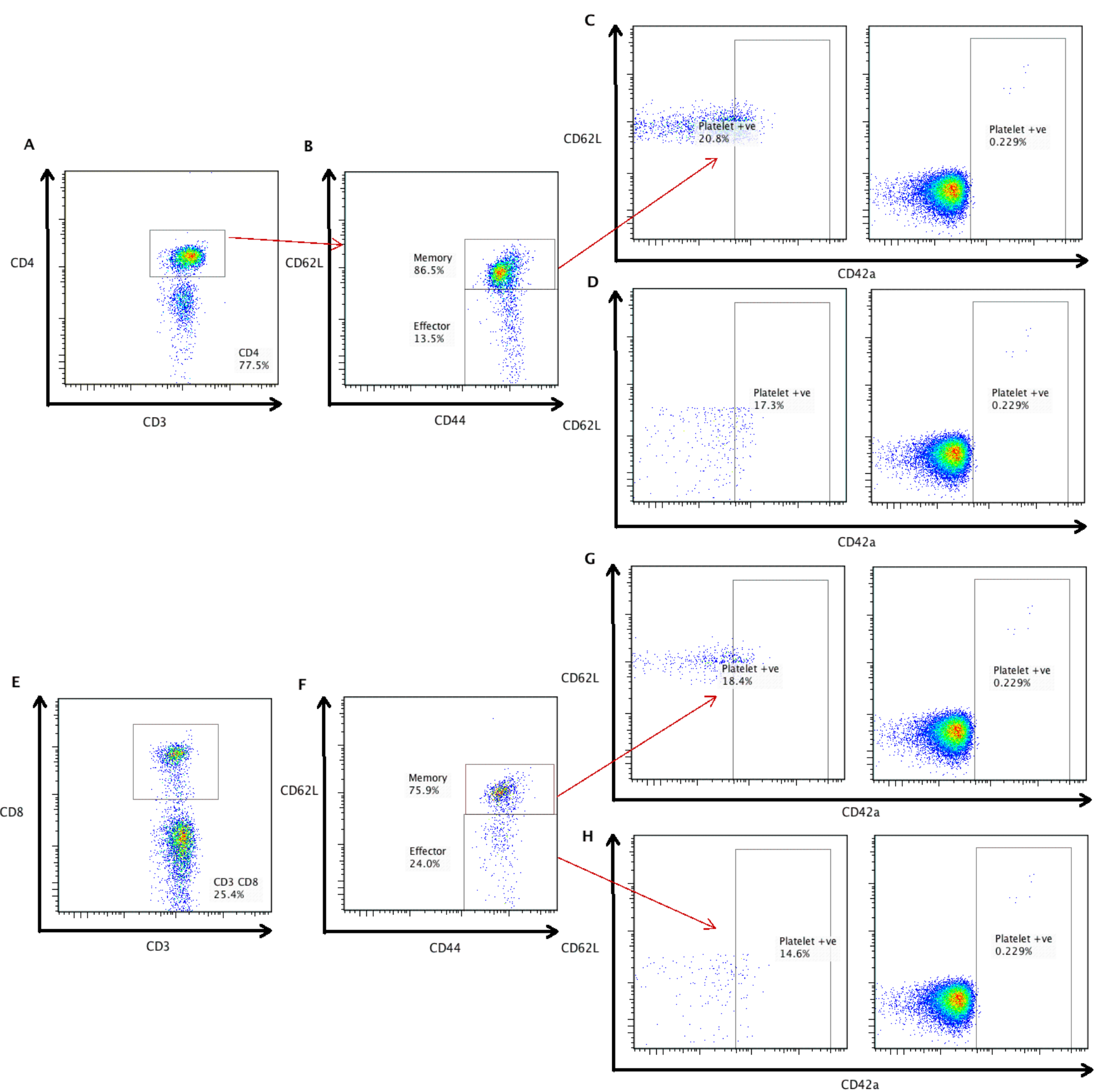

Figure 7-2 Gating strategy to identify activation phenotype of CD4 and CD8 T cells

CD4 and CD8 T cells were then identified by their expression of CD4 (A) and CD8 (E) respectively The cell markers $\mathrm{CD} 62 \mathrm{~L}$ and $\mathrm{CD} 44$ were used to identify memory and effector $\mathrm{T}$ cells on pre gated CD4 (B) and CD8 populations (F). The platelet marker CD42a was used to identify memory $T$ cell- platelet conjugates $(C \& G)$ and effector T cell-platelet conjugates ( $D$ \& $\mathrm{H})$ as compared to isotype control (right panel). 


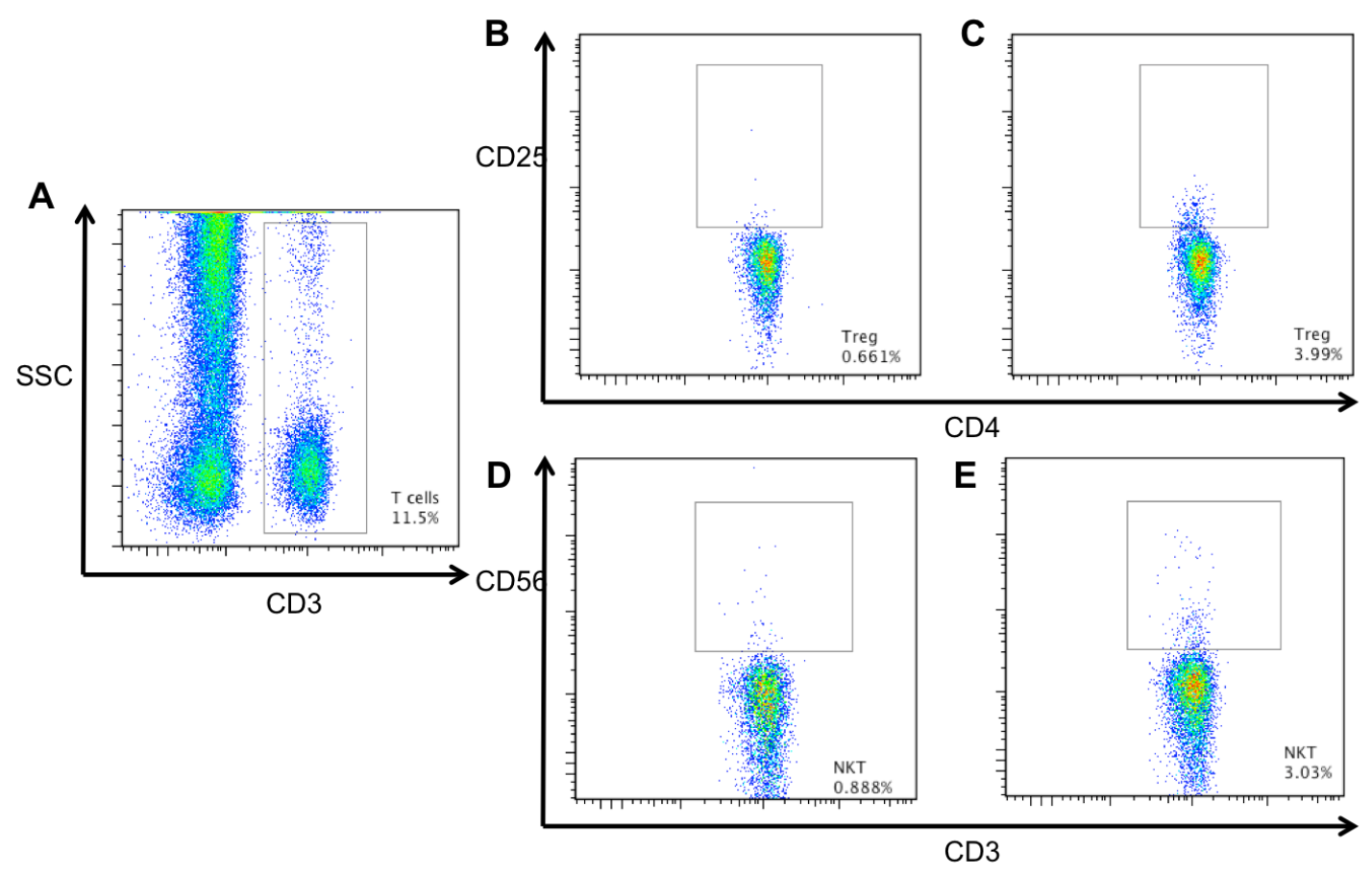

Figure 7-3 Gating strategies to identify T regulatory (Treg) cells and Natural Killer T cells (NKT).

T cells were identified by their SSC properties and expression of CD3 (A). Treg cells were identified by their expression of CD4 and CD25 (C) as compared to isotype control (B). NKT cells were identified by the expression of CD56 (D) as compared to the isotype control (C).

\subsubsection{Statistics}

Platelet-T cell conjugate data was normally distributed as confirmed by D'Agostino \& Pearson normality testing. Platelet-T cell conjugates are expressed at mean percentages \pm the standard deviation. Platelet-T cell conjugates were compared between healthy and vascular cohorts using student $t$ test. Statistical analysis and graphs generated using GraphPad prism 5 (GraphPad Software Inc., La Jolla, CA, USA). 


\subsection{Results}

\subsubsection{Demographics}

The healthy volunteer group was relatively young with a mean age of 31 and did not have any known cardiovascular disease or risk factors as per the inclusion/exclusion criteria (Table 7-2). The vascular patient group was more than twice the age of the healthy volunteers at 77 and displayed multiple cardiovascular risk factors and 3 of the patients had documented ischaemic heart disease or previous stroke. Only 2 patients were receiving statin therapy and several were on blood pressure lowering agents. The two populations were sex matched with 4 males in each group.

Table 7-2 Demographics of healthy volunteers and vascular patients

\begin{tabular}{|l|l|l|}
\hline & $\begin{array}{l}\text { Healthy volunteers } \\
(\mathrm{n}=10)\end{array}$ & $\begin{array}{l}\text { Vascular patients } \\
(\mathrm{n}=10)\end{array}$ \\
\hline Male, $\mathrm{n}$ & 4 & 4 \\
\hline Age & $31 \pm 9$ & $77 \pm 8$ \\
\hline Current Smoker & 0 & 4 \\
\hline Diabetes & 0 & 4 \\
\hline Hypertension & 0 & 6 \\
\hline Hyperlipidaemia & 0 & 2 \\
\hline Ischaemic heart disease & 0 & 2 \\
\hline Previous stroke & 0 & 1 \\
\hline Medications & \multicolumn{2}{|l|}{} \\
\hline Statin & 0 & 2 \\
\hline Beta blocker & 0 & 3 \\
\hline ACE inhibitor & 0 & 2 \\
\hline Calcium channel blocker & 0 & 1 \\
\hline Diuretic & 0 & 3 \\
\hline
\end{tabular}

\subsubsection{Platelet-T cell conjugates}

Firstly, the overall T cell population was analysed and total platelet-T cell conjugates were compared between the two participant groups. The percentage of platelet-T cell conjugates was significantly increased in patients with vascular disease $(37.7 \% \pm 10 \%)$ compared to healthy controls $(23.9 \% \pm 8.7 \%, p=0.005$, Figure $7-4)$. 


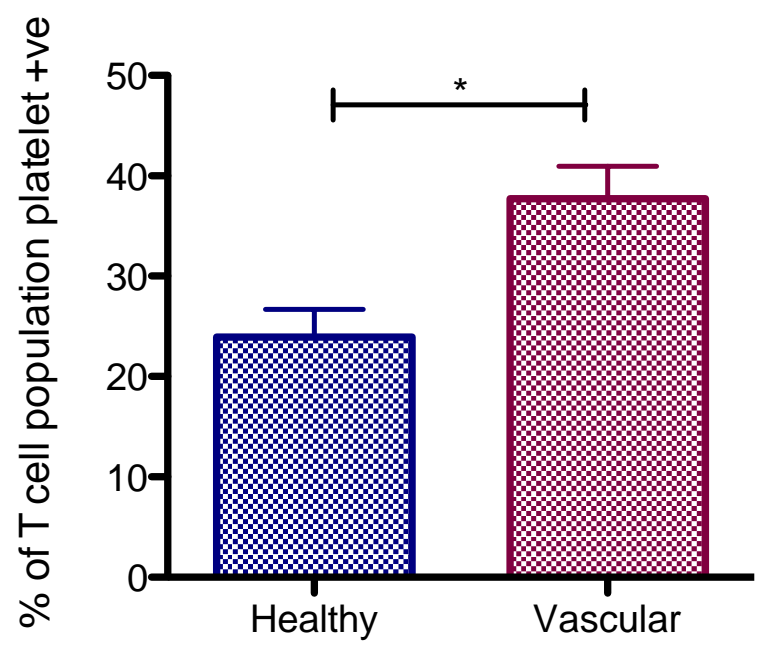

Figure 7-4 Platelet-T cell conjugates

Whole blood from healthy volunteers $(n=10)$ and vascular patients $(n=10)$ were stained with anti-CD42a and anti-CD3 antibodies to identify platelet-T cell conjugates using flow cytometry. Platelet-T cell conjugates are presented as the \% of T cells positive for the CD42a marker. Data is plotted for all 10 patients in each group as mean \pm SD in healthy volunteers and patients with vascular disease. Student T test * $\mathrm{p}=0.005$.

\subsubsection{CD4 T cell interactions}

To investigate differences in platelet interactions with T cell subgroups, we initially looked at CD4 T cell populations and found significant changes in this subgroup between patient groups. The majority of $\mathrm{T}$ cells in both healthy volunteers and patients with vascular disease expressed CD4, comprising approximately $70 \%$ of the $T$ cells (Figure 7-5A). Although there was no significant difference in the proportion of CD4 T cells between healthy and vascular patients, the level of platelet-CD4 T cell conjugates was significantly higher in the vascular patients (Figure 7-5B). Platelet-CD4 T cell conjugates increased by $40 \%$ from $26.6 \% \pm 5.9 \%$ in healthy volunteers to $37.6 \%$ $\pm 7.9 \%$ in vascular patients, $p=0.004$. There were also differences in the activation phenotype of the CD4 T cells between the two patients groups. In the healthy volunteers, the majority of CD4 T cells were of a memory phenotype with a smaller proportion exhibiting effector phenotype markers (Figure 7-5C). In the vascular patients the majority of CD4 T cells were of an effector phenotype resulting in a 2 fold increase from healthy levels $(31.8 \% \pm 7 \%$ to $64.8 \% \pm 11 \%, p<0.0001)$. Accordingly there was a substantial drop in memory CD4 cells in vascular patient samples compared to healthy volunteers $(61.3 \% \pm 11 \%$ to $33.3 \% \pm 13 \%$. $\mathrm{P}<0.0001)$. Although 
there was a large shift in the activation state of CD4 T cells between patient groups, there was no significant difference in the level of platelet-T cell conjugates between these different activation phenotypes (Figure 7-5D).

A

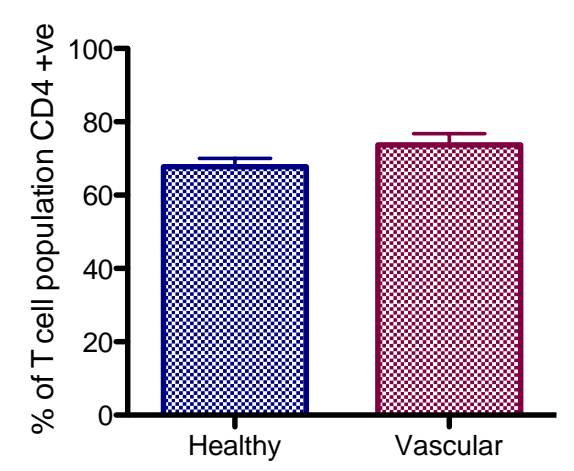

C

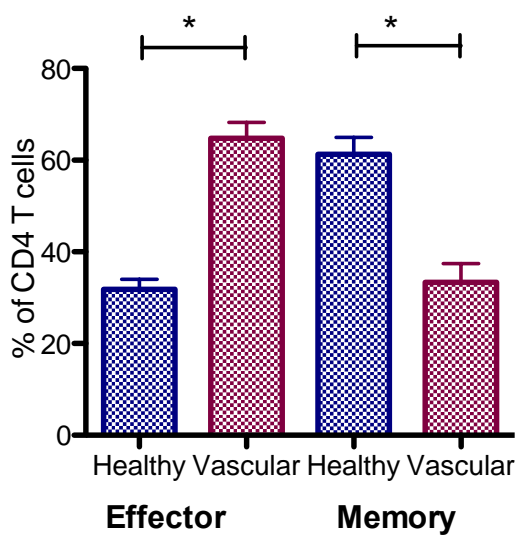

B

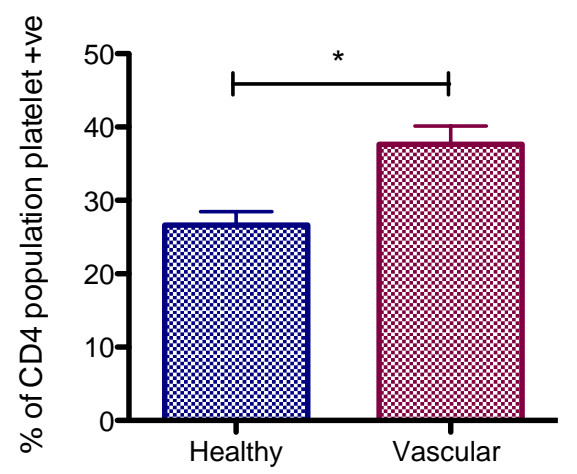

D

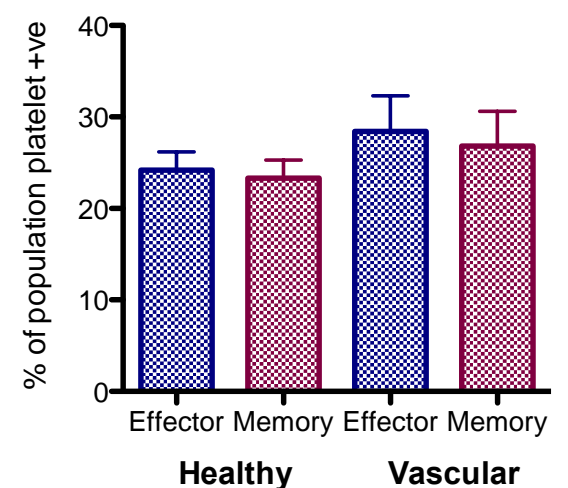

Figure 7-5 CD4 T cell interactions with platelets

Whole blood from healthy volunteers $(n=10)$ and vascular patients $(n=10)$ were stained with anti-CD42a, anti-CD3, anti-CD4, anti-CD62L and anti-CD44 antibodies to identify platelet-CD4 $T$ cell conjugates and CD4 activation phenotypes using flow cytometry. These antibodies were used to specifically identify: the proportion of T cell population expressing CD4 (A), plateletCD4 T cell conjugates (B), the proportion of CD4 T cells displaying effector and memory activation phenotypes $(C)$ and the proportion of effector and memory CD4 cells that are platelet bound (D) in healthy volunteers and vascular patients. Data is plotted for all 10 patients in each group as mean \pm SD Student T test ${ }^{*} p<0.001$.

A subset of CD4 T cells; T regulatory (Treg) cells were investigated and found to be in low circulating numbers making up on average $1.1 \% \pm 0.7 \%$ of CD4 positive cells. In vascular patients the proportion of Treg cells doubled to a mean of $2.1 \% \pm 1.2 \%(p=$ 0.04, Figure 7-6A). However, the level of platelet conjugates in the Treg population did not significantly change between the healthy volunteers and vascular patients $(28.3 \% \pm 9.2 \%$ vs. $35.84 \% \pm 12.5 \%, p=0.14$, Figure $7-6 \mathrm{~B})$ 
A

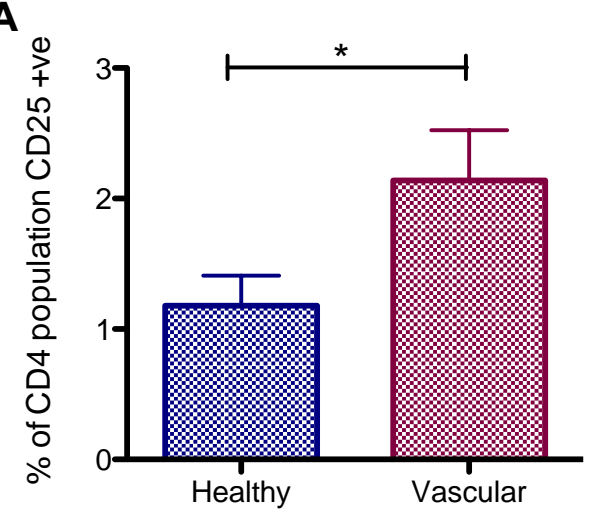

B

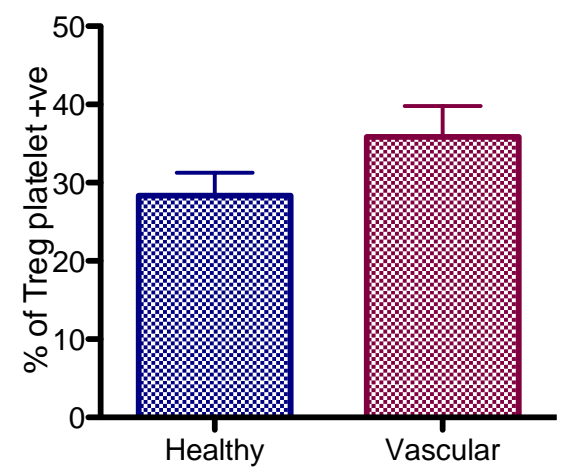

Figure 7-6 T regulatory (Treg) cell interactions with platelets

Whole blood from healthy volunteers $(n=10)$ and vascular patients $(n=10)$ were stained with anti-CD42a, anti-CD3, anti-CD4 and anti-CD25 antibodies to identify Treg cells and plateletTreg conjugates using flow cytometry. Data shows the proportion of CD4 T cells expressing the Treg marker CD25 (A) and the level of platelet-Treg conjugates $(B)$ in healthy volunteers and vascular patients. Data is plotted for all 10 patients in each group as mean \pm SD. Student $T$ test ${ }^{*} p=0.04$

\subsubsection{CD8 T cell interactions}

Platelet interactions were also investigated in CD8 T cells. Similar to the CD4 T cell population, the proportion of CD8 T cells did not change between healthy volunteers and vascular patients but made up a smaller proportion at 22\% (Figure 7-7A). In contrast to platelet-CD4 T cell interactions, the level of platelet-CD8 T cell conjugates were not significantly elevated in vascular patients ( $33 \% \pm 9 \%)$ compared to healthy volunteers (31\% $\pm 8 \%$, Figure 7-7B). The activation phenotypes were also investigated in CD8 T cells. Similar to the observed activation phenotypes of CD4 cells, there was a 1.4 fold increase in the effector phenotype in vascular patient samples $(35.4 \% \pm 10 \%)$ compared to healthy volunteers $(25 \% \pm 9 \%, p=0.03)$. However, there was no difference in the memory CD8 cells between the two groups and these remained the predominant activation phenotype in both healthy volunteers and vascular patients (Figure 7-7C). Furthermore, the level of platelet-CD8 conjugates did not differ between effector/memory phenotypes or patient population (Figure 7-7D). 
A

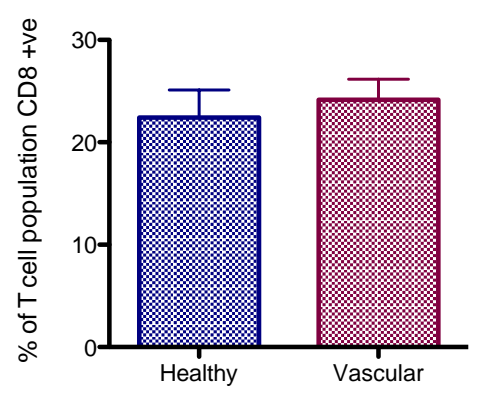

C

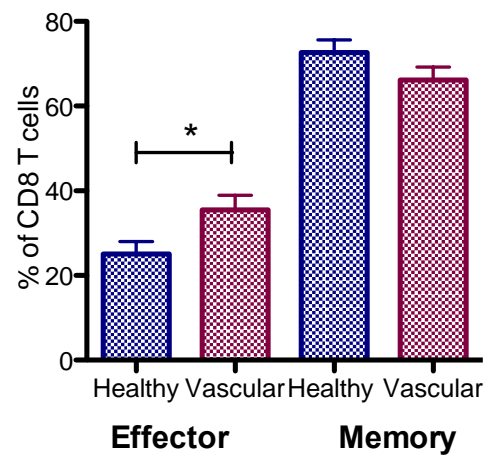

B

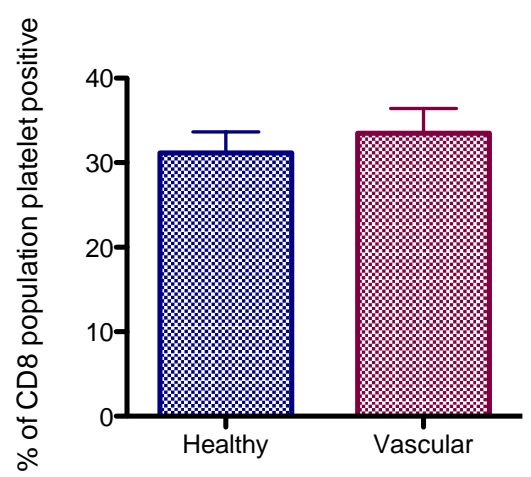

D

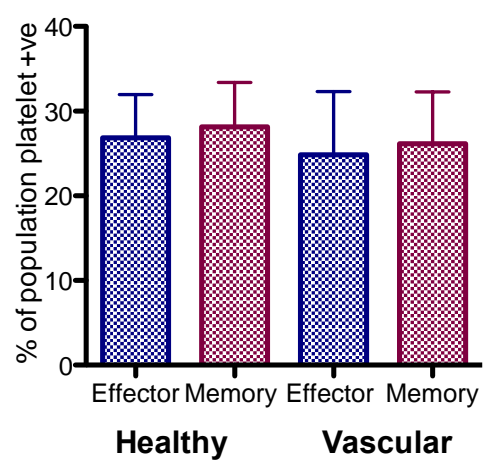

Figure 7-7 CD8 T cell interactions with platelets.

Whole blood from healthy volunteers $(n=10)$ and vascular patients $(n=10)$ were stained with anti-CD42a, anti-CD3, anti-CD8, anti-CD62L and anti-CD44 antibodies to identify platelet-CD8 $T$ cell conjugates and CD8 activation phenotypes using flow cytometry. These antibodies were used to specifically identify: the proportion of the T cell population expressing CD8 (A), plateletCD8 T cell conjugates (B), the proportion of CD8 T cells displaying effector and memory activation phenotypes $(C)$ and the proportion of effector and memory CD8 cells that are platelet bound $(D)$ in healthy volunteers and vascular patients. Data is plotted for all 10 patients in each group as mean \pm SD. Student $T$ test ${ }^{*} p<0.05$.

\subsubsection{Natural Killer T cells}

Natural killer T (NKT) cells were found to constitute approximately $2.8 \% \pm 1.9 \%$ of $\mathrm{T}$ cells in healthy volunteers. NKT cells were more populous in vascular patients with an increase of $40 \%$ compared to healthy levels $(4.5 \% \pm 2 \%)$, however this difference was not statistically significant $(P=0.06$, Figure $7-8 A)$. Furthermore, there was a trend of increased platelet binding to NKT cells in vascular patients compared to healthy controls ( $25 \% \pm 7 \%$ vs. $35 \% \pm 15 \%, p=0.08$, Figure $7-8 B)$. 
A

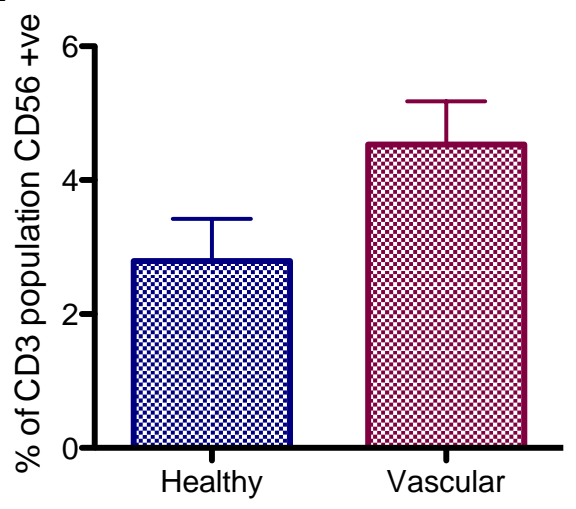

B

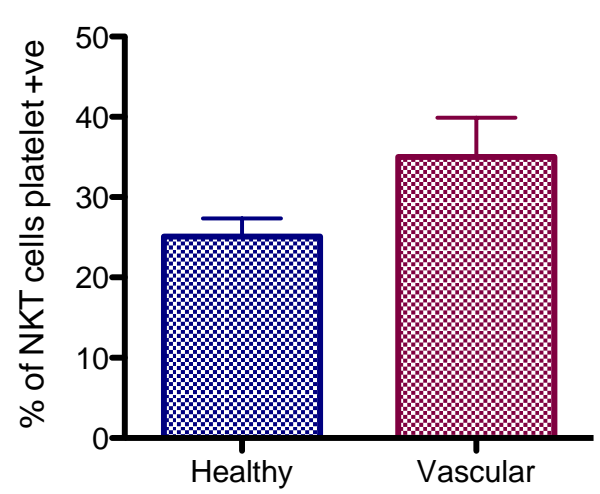

Figure 7-8 Natural Killer T (NKT) cell interactions with platelets

Whole blood from healthy volunteers $(n=10)$ and vascular patients $(n=10)$ were stained with anti-CD42a, anti-CD3 and anti-CD56 antibodies to identify NKT cells and platelet-NKT conjugates using flow cytometry. Data shows the proportion of $T$ cells expressing the NKT marlker CD56 (A) and the level of platelet-NKT conjugates (B) in healthy volunteers and vascular patients. Data is plotted for all 10 patients in each group as mean \pm SD. Student $T$ test - ns. 


\subsection{Discussion}

This study investigated platelet-T cell interactions in a healthy and an atherosclerotic inflammatory disease state and found significant differences between the two groups. Platelet-T cell conjugates were found to be more prevalent in vascular patients. Platelet binding to CD4 T cells also occurred more frequently in vascular patients when compared to healthy volunteers. However, there was no significant change in platelets binding to CD8 or NKT cells between the two groups. The activation phenotypes of CD4 T cells also changed dramatically, with a predominant memory phenotype seen in healthy volunteers switching to an effector response in vascular patients. This switch in activation was not observed in the CD8 T cells. For both the CD4 and CD8 T cell subgroups, there was no change in platelet binding to the different activation phenotypes or change between the healthy volunteers and vascular patients.

We observed a high level of platelet-T cell conjugates in vascular patients at an average of $37 \%$ compared to $20 \%$ in healthy volunteers. This is the first study to look at platelet-T cell conjugates in atherosclerotic vascular disease in humans compared to healthy volunteers specifically. Previous studies have investigated other immune cells interacting with platelets such as circulating platelet-neutrophil, plateletmonocyte and overall platelet-leukocyte conjugates in atherosclerosis, diabetes, cerebral stroke and other inflammatory states $(406,407)$. These studies also observed a significant increase in platelet-immune cell conjugates but did not investigate the particular differences in the T cell population.

The higher level of platelet-T cell conjugates found in the vascular disease population may be due to several factors influencing both the platelets and T cells reactivity. Resting platelets do not express P-selectin or CD40, but upon activation these two ligands are quickly trafficked to the surface. In advanced atherosclerosis such as vascular disease, platelets display a higher activity and express significant levels of both ligands which are the main linkers to attach to $T$ cells and potentially influence function and differentiation (78). Stimulation of platelets with classical agonists such as ADP and thromboxane $A_{2}$ has been shown to increase platelet-leukocyte 
conjugates (408) in healthy and ACS populations however this has not been confirmed in T cells.

The communication between platelets and T cells is most likely bidirectional and the activation state of the $\mathrm{T}$ cell will also influence its ability to bind platelets. In vitro stimulation of human $T$ cells with anti-CD3/anti-CD28 showed an enhanced CD40L surface expression on T cells leading to more potential communication with platelets through the CD40-CD40L system (409). In a further study done in healthy volunteers, expression of this ligand on T cells resulted in contact mediated activation of platelets via the CD40 dependent pathway resulting in RANTES release from platelets, which can influence T cell trafficking (410). Additionally, a study conducted in healthy volunteers found T cells that were larger in size and more granular coupled with high expression levels of the activation marker CD69, had more platelets attached compared to the smaller less active T cells (411). As this study looked purely at the level of interaction between platelets and T cells and we did not block the cells activity we cannot determine which cell is determining the interaction. Although, we would hypothesise that activation of either cell will enhance platelet-T cell conjugation.

Other disease states, such as diabetes can also influence platelet and T cell function. A high proportion of patients with atherosclerosis, particularly those with lower extremity vascular diseases, have diabetes and as reviewed in section 1.4.4.3, this highly inflamed state can produce abnormally reactive and pro-inflammatory platelets. This vascular cohort also had other co-morbidities and underlying illness, including hyperlipidaemia, hypertension, and smoking which could be nurturing both pro and anti-inflammatory states. Due to these other conditions, many of the patients were on several drug therapies, which may also be influencing platelet and $\mathrm{T}$ cell responses. Surprisingly, at the time of enrolment only 2 of the vascular cohort were receiving statin therapy, which as a front line therapy for vascular disease has been shown to have profound effects on not only lipid levels but the immune system as well (412). 
The level of platelet-T cell conjugates observed in our healthy donor population was relatively high at $20 \%$ compared to other reports of platelet-lymphocyte conjugates in healthy volunteers $(3-15 \%)(411,413)$. This can be partially explained by the different parameters other studies expressed their platelet-leukocyte populations and the experimental conditions used. Several studies have expressed plateletlymphocyte conjugates based on size and CD45 expression, which includes both T and B cells. As B cells have shown to have a low propensity to bind with platelets (414), this may decrease overall averages reported. Several studies have used sodium citrate as their anticoagulant for blood collection and as shown in Chapter 2, citrate can cause a decrease in platelet reactivity due to the depletion of calcium, which may potentially result in an artificially low platelet-T cell conjugate level. Studies investigating platelet-leukocyte binding in different anticoagulants, found a dramatic reduction in citrated blood compared to bivalirudin and heparin, due the calcium dependent nature of P-selectin binding with PSGL-1 (417).

Within the T cell subgroups, we found interesting changes in the T helper cells that were not displayed in the cytotoxic CD8 cells. Platelets binding with CD4 cells occurred more frequently in vascular patients compared to CD8 or NKT cells. CD4 T helper cells are abundant in atherosclerotic plaques and their important role in atherogenesis has been highlighted by earlier animal studies showing that transfer of CD4 T cells aggravates (418), whereas CD4 deficiency attenuates (417) development in ApoE knockout mice. This significant increase in platelet-CD4 T cell binding observed in patients with vascular disease, could indicate important communications occurring between these two cell types in atherosclerosis.

The predominant activation phenotype of CD4 T cells shifted from a memory phenotype in healthy donors to an effector phenotype in vascular patients. CD4 T effector cells are antigen-experienced cells that offer immediate effector functions and migrate into plaques to sustain inflammation (418). A study looking at CD4 naïve, effector and memory cells found high levels of effector cells were associated with a greater intima-media thickness of the common carotid artery and were more common in patients with chronic angina or ACS compared to healthy controls (418). However, 
with this change in activation we did not see a significant difference in platelet binding between the different activation states or patient groups. Furthermore we observed an increase in Tregs in vascular patients but no increase in platelet binding. This difference may be partially due to the smaller cell numbers and statistical power when analyzing platelet binding within CD4 subgroups. It also could indicate the platelets' effect on CD4 T cells is mediated through mechanisms other than platelet binding.

Platelets can exert their effects on immune cells not only through cell-to-cell contact but also through powerful soluble mediators. Platelets release many soluble factors, which can have mitogenic effects on a diverse range of cells including lymphocytes. In vitro human and animal models have demonstrated the soluble factors RANTES and platelet-derived growth factor (PDGF) promote proliferation of CD4 T cells $(419,420)$. Other soluble mediators such as Platelet factor 4 (PF4), TGF $\beta$ and thromboxane A2 have more complex actions on proliferation and either promote or inhibit proliferation depending on the local environment and the CD4 subset that is being targeted (423). Similarly, platelets release a multitude of cytokines, chemokines and growth factors that effect CD4 T cell differentiation and cytokine production. As with proliferation, these interactions are complex and each CD4 T cell subset is affected differently. Th1 cells appear to be highly stimulated by platelets via IL-1ß (422), RANTES (423) and monocyte chemoattractant protein-1 (MCP-1) (424) whereas Th2 are either stimulated, barely affected or inhibited by platelet contact depending on the environment (423). Interestingly, Tregs are also stimulated by platelets particularly through TGF $\beta(423,425)$ and PF4 $(426)$. Th17 are a relatively new subtype of CD4 T cells to be investigated and platelets act to promote differentiation and cytokine production through IL-1 $\beta$ and TGF $\beta$ (422). But as many of these studies that have investigated the effect of isolated mediators on $\mathrm{T}$ cell differentiation and function have been conducted in vitro, crucial in vivo information is lacking on how platelets act on CD4 T cells within the complex milieu of atherosclerosis.

As this study looked at the overall CD4 T cell population and not the individual subsets, we cannot determine whether there were significant differences in binding between the subsets in a healthy state or atherosclerosis. As highlighted in section 1.2.2, Th1 
cells are abundant in plaques and promote a pro-inflammatory environment. Whereas Tregs suppress inflammation and their absence is linked to progressive and unstable plaques. However, all CD4 T cell subsets are present in plaques with varying numbers. Therefore understanding how platelets interact with these cells, the consequences of these interactions on the immune system and how this is modified by existing antiplatelet agents is important as it will allow us to understand potential mechanisms of action of existing drugs and may allow us to identify additional targets for novel therapies.

Unlike the CD4 T cell population there were few changes in the CD8 cytotoxic T cells between healthy donors and vascular patients. There was a $40 \%$ increase in effector CD8 T cells, but this activation phenotype did not predominate. There was no increase in platelet binding to CD8 T. CD8 T cells are present in atherosclerotic plaques and have been identified in early lesions in mouse models, yet their role in atherosclerosis remains unresolved. A recent study looking at CD8 T cells in a mouse model of atherosclerosis concluded $\mathrm{CD}^{+} \mathrm{T}$ lymphocytes promote the development of vulnerable atherosclerotic plaques by perforin- and granzyme B-mediated apoptosis of macrophages, smooth muscle cells, and endothelial cells that, in turn, leads to necrotic core formation and further augments inflammation by TNF- $\alpha$ secretion (427). However, there is limited evidence to confirm this finding in humans. The increase in CD8 effector $T$ cells observed in the vascular group may represent more active CD8 T cells in atherosclerosis promoting inflammation. Platelets have been reported to interact with and enhance CD8 T activity; particularly in adenovirus infections, causing IFN- $\gamma$ production and increasing the cytolytic capacity of CD8 T cells (407). Although our study would suggest that platelets are not influencing CD8 T cell function in vascular disease.

Our study observed a $40 \%$ increase in Natural Killer T (NKT) cells in vascular disease however this trend was not statistically significant. NKT cells are a unique subset of T lymphocytes that share surface markers from both conventional T cells and NK cells. Although NKT cells are associated with decreasing inflammation and immune responses in autoimmune disease and cancer (430), their role in atherosclerosis has 
been found to be pro-atherogenic especially in early lesional development. Unlike conventional T cells that only recognize peptide antigens, NKT cells can recognize glycolipid antigens as well and it is this ability that has been hypothesized to promote the pro-atherogenic response of NKT cells in a typically high ox-LDL environment (431). With the increase in NKT cells in our vascular cohort we also observed a trend to increased platelet binding. The potential for platelets and NKT to interact are practically unknown in either healthy donors or patients with atherosclerosis. Studies investigating Natural Killer (NK) function have shown platelets can inhibit NK cell's anti-tumor cytolytic ability, thereby increasing tumor metastasis in mouse models (432) and as discussed previously platelets exert their influence on T cells in a host of different ways. Therefore, this finding could signify a novel interaction of platelets and NKT cells in atherosclerosis that warrants further investigation.

\subsection{Limitations}

As we designed this study to look specifically at platelet-T cell interactions, we did not include markers of platelet-monocyte or platelet-neutrophil conjugates which have been well documented and investigated in these two populations and could have acted as a positive control for the assay in our cohorts. However, as we were interested in the changes between the two populations using the same standardised procedure and not at platelet-T cell conjugates as a biomarker for disease, the need for this positive control is reduced. The study population was relatively small with 10 people per group and a larger cohort would have allowed more statistical power for subgroup analysis. However we did find significant differences between the healthy donors and vascular group, which has highlighted areas of interest to pursue further. The average age of the healthy volunteer group was significantly lower than the vascular population, which may have impacted on our findings. However, to ensure the healthy group were free of risk factors and were unlikely to have occult atherosclerosis, this difference in age was necessary to investigate our aims. Similar to other vascular disease populations, several of the patients had other co-morbidities such as diabetes, hyperlipidaemia and hypertension and receiving treatments that we could not rule out as factors influencing platelet-T cell conjugates irrespective of atherosclerosis. But as this disease is complex and the interactions between platelets 
and immune cells change dramatically depending on the physiological and pathological microenvironment, studying these interactions in a real world in vivo setting is imperative to advancing our understanding of atherosclerosis.

\subsection{Conclusion}

In this study we observed an increase in platelet-T cell conjugates in vascular patients compared to healthy donors. Platelets also preferentially bound to CD4 T and NKT cells in vascular disease, which was not observed in the CD8 T cell subgroup. The CD4 T cell subgroup also exhibited changes in the activation phenotype, shifting from a predominantly memory phenotype in healthy patients to an effector response in vascular disease, which again was not observed in the CD8 cells. These changes in the CD4 T cell population coupled with our existing knowledge of the important role of CD4 T cells in atherosclerosis, raises questions on how platelets may be influencing the different subtypes of helper $T$ cells to influence disease progression and what impact inhibiting these interactions has on the immune system. 
CHAPTER 7 


\section{${ }_{8}$ Chapter 8 - The effect of platelets on CD4 $\mathrm{T}$ cell differentiation}




\subsection{Introduction}

As discussed in Chapter 7, CD4 T cells are major orchestrators of atherosclerotic lesion progression, and platelets may provide key interactions with CD4 T cells that influence their response. However, because different subsets of CD4 T cells may have either pro-inflammatory or regulatory functions in atherosclerosis, it is unknown how platelets may impact each of these different functional subsets. The inhibition of platelets with clopidogrel has been associated with reductions in CRP levels and decreased expression of CD40L and P-selectin in a variety of disease states, including cardiovascular disease, cerebrovascular disease, diabetes, and renal transplantation (433). This anti-inflammatory response may potentially be due to modulation of leukocyte responses by platelet inhibition, and in particular, could involve the inhibition of platelet interactions with pro-inflammatory $\mathrm{T}$ cells or possibly augmentation of the anti-inflammatory activity of Tregs.

Antiplatelet therapy is a cornerstone treatment in patients with CAD and PVD due to their known ability to prevent platelet aggregation and reduce thrombotic risk. This has been our main focus in the first series of studies. However, antiplatelet therapy may be providing protection from adverse events through other mechanisms other than thrombosis.

Therefore this chapter investigates the impact of platelet interactions on CD4 T cell differentiation and the effect of antiplatelet therapy on platelet-T cell interactions. This study was designed to investigate 1 ) how platelets could modify CD4 T cell subset differentiation and 2) if and how this differentiation was changed after antiplatelet therapy. Therefore, to exclude any variance in the immune response due to disease state, concurrent medication use and increasing age that is often present in patients with atherosclerosis, we decided to conduct the study in healthy volunteers. 
The specific aims of the study were:

1. To examine the impact of CD4 T cell-platelet interactions on IFN- $\gamma$ production.

2. To investigate the impact of CD4 T cell-platelet interactions on CD4 T cell differentiation, specifically Th1, Th17 and Treg subsets.

3. To investigate the effect of inhibiting platelet-T cell interactions in-vivo on IFN- $\gamma$ production and $\mathrm{T}$ cell differentiation using the $\mathrm{P} 2 \mathrm{Y} 12$ antagonist, prasugrel.

\subsection{Methods}

\subsubsection{Study population}

Participants enrolled in the study were between the ages of $18-50$ years with no known cardiovascular disease. Exclusion criteria included diabetes, smokers, an acute illness within the preceding six weeks and those who were treated with cardiovascular medication including antiplatelet therapies, non-steroidal anti-inflammatory drugs (NSAID) or immune modulating medications. All participants provided written informed consent and the study was reviewed and approved by the Central Regional Ethics Committee (12/CEN/59). The study was also registered with the Australian New Zealand Clinical Trials Registry (registration number ACTRN12612001157864).

\subsubsection{Study design and drug treatment}

This was a prospective, double-blind, placebo-controlled, randomized crossover trial. Subjects were randomized to receive either $60 \mathrm{mg}$ of prasugrel (Group A) followed by $10 \mathrm{mg} /$ day prasugrel for 6 days or placebo (Group B) (Figure 8-1). After the first treatment there was a washout period of 21 days and participants were crossed over to the other study drug. An independent cardiology research nurse conducted randomization and drug administration. 


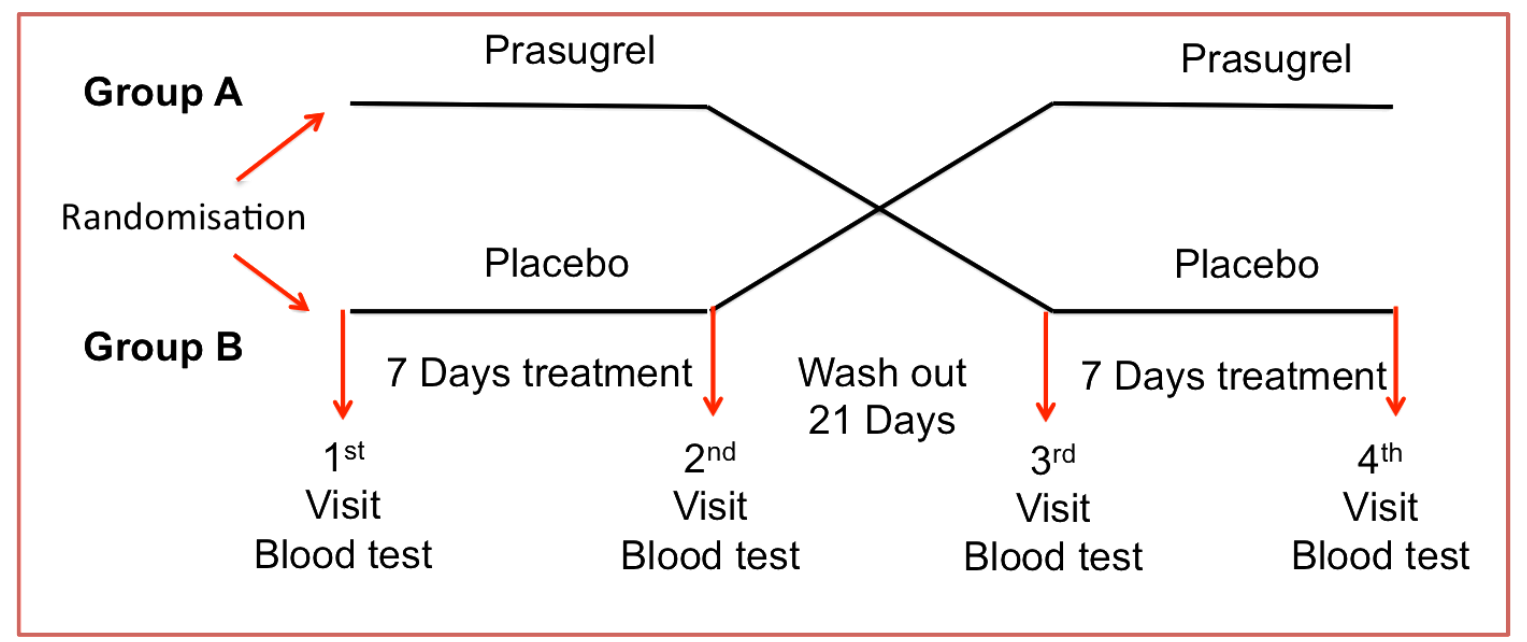

Figure 8-1 Study cross over protocol

\subsubsection{Blood sampling and Multiplate testing}

Blood was drawn before and after each drug treatment from a peripheral arm vein as described previously in Chapter 2.2.2. Thirty-five $\mathrm{ml}$ of blood was drawn into sodium heparin tubes (158 USP unites, BD Biosciences, NJ, USA) for CD4 T cell isolation. A single hirudin tube $(25 \mu \mathrm{g} / \mathrm{ml}$, Dynabyte, Munich, Germany) was also obtained for isolation of autologous platelets and platelet function testing using the Multiplate, as described in Chapter 2.2.2 to assess adequate antiplatelet therapy was achieved.

\subsubsection{Peripheral blood mononuclear cell (PBMC) isolation}

All cell work was carried out in a class II purifier biological safety cabinet (LABCONCO, MO, USA) with HEPE air filters. Before use, sterilization with ultraviolet (UV) light (1390 mW/m2) for $15 \mathrm{~min}$ was carried out and all surfaces were wiped down with $70 \%$ ethanol. Blood was diluted 2.3 fold in sterile PBS and carefully layered on top of RT Histopaque-1077 Hybri Max (Sigma, MO, USA) in 15 ml Falcon tubes (BD Biosciences, NJ, USA) with a 1:1 ration of Histopaque to whole blood. The tubes were then spun at $400 \times \mathrm{g}$ for 30 minutes at RT. The upper plasma layer was aspirated and discarded to within $0.5 \mathrm{~cm}$ of the opaque interface containing the peripheral blood mononuclear cells (PBMCs). The buffy coat layer was then carefully transferred to a separate tube and washed twice with $10 \mathrm{ml}$ of dPBS, mixed with inversion and centrifuged at $250 \mathrm{x}$ 
g for 5 minutes. Cells were counted using a hemocytometer and $0.4 \%$ tryphan blue (Sigma, MO, USA) and resuspended at $5 \times 10^{7}$ cells $/ \mathrm{ml}$ in isolation buffer (Appendix 1 ).

\subsubsection{CD4 T cell isolation}

CD4 T cells were isolated using the Dynabeads Untouched Human CD4 T cell kit (Life Technologies, CA, USA). Following the manufacturers instructions, $100 \mu \mathrm{L}$ of FCS and $100 \mu \mathrm{L}$ of antibody mix was added to the aliquot of isolated PBMCs and incubated for 20 minutes at $4^{\circ} \mathrm{C}$. Cells were then washed with $4 \mathrm{ml}$ of isolation buffer and mixed by inversion several times before being centrifuged at $350 \times \mathrm{g}$ for 8 minutes at $5^{\circ} \mathrm{C}$. The washed pellet of cells was resuspended in $500 \mu \mathrm{L}$ of IB. $500 \mu \mathrm{L}$ of pre-washed Dynabeads (Life Technologies) were added to the cell suspension and the sample was incubated for 15 minutes at RT with gentle tilting and rotation. Following incubation, $4 \mathrm{ml}$ of isolation buffer was added to the bead-cell mix and the bead-bound cells were resuspended by pipetting the sample thoroughly with a $100 \mu \mathrm{L}$ tipped pipette whilst avoiding foaming. The tube was then placed in the Dynamag magnet (Life Technologies) for 2 minutes and the supernatant containing the isolated CD4 T cells was transferred to a fresh tube. A further $4 \mathrm{ml}$ of isolation buffer was added to the tube containing the bead-bound cells, repeating the resuspension and aspiration of remaining CD4 T cells. The CD4 T cells were counted and washed before resuspending in dPBS at $2 \times 10^{6}$ cells $/ \mathrm{ml}$.

\subsubsection{Isolation of autologous platelets}

Platelets were isolated from hirudin anticoagulated blood by centrifuging at $200 \mathrm{xg}$ for 20 minutes at RT. The upper 2/3 of platelet-rich-plasma (PRP) was collected and a platelet count was determined using the XE-2100 hematology analyser (Sysmex, $\mathrm{GmbH}$, Germany) operated and located in the Wellington Hospital Medical Laboratory. PRP was centrifuged at 1,000 $\mathrm{x}$ for 15 minutes at RT and resuspended in CTCM at $2 \times 10^{8}$ cells $/ \mathrm{ml}$. 


\subsubsection{Cell culture}

T cells were resuspended in CTCM and plated in 96-well tissue culture $U$ bottom plates (BD Biosciences, NJ, USA) with $2 \times 10^{6} \mathrm{~T}$ cells per well. For stimulated samples, the T cells were plated in 96 well anti-human CD3 T cell activation plates (BD Biosciences, NJ, USA) with the addition of $0.3 \mathrm{ug} / \mathrm{ml}$ of purified NA/LE mouse anti-human CD28, clone CD28.2 (BD Biosciences, NJ, USA). For platelet-T cell co-cultures, $1 \times 10^{7}$ platelets resuspended in CTCM were added to sample wells to give a final ratio of 1:250 of T cells to platelets. All samples were cultured in a Sanyo $\mathrm{CO}_{2}$ incubator (Global Science, $\mathrm{PA}$, USA) for 48 hours in a humid environment at $37^{\circ} \mathrm{C}$, supplemented with $5 \% \mathrm{CO}_{2}$. For the last 5 hours of incubation BD GolgiStop (BD Biosciences, NJ, USA) was added to all samples and anti-CD3/CD28-stimulated CD4 T cells were challenged with PMA (50 ng/ml)/lonomycin $(1 \mu \mathrm{g} / \mathrm{ml})$.

\subsubsection{CD4 T cell phenotyping}

Samples were transferred to eppendorf tubes after 48 hours of incubation and cells were spun out of media at $250 \times \mathrm{g}$ for 5 minutes. Supernatant from cell cultures was aspirated and frozen for IFN- $\gamma$ quantification. Cells were washed in $500 \mu \mathrm{L}$ of PBS, spun at $250 \times \mathrm{g}$ for 5 minutes and the supernatant removed to leave $50 \mu \mathrm{L}$ of resuspended cells. Fixable viability dye eFlour 780 (eBioscience, CA, USA) was diluted 1:5000 and added to cells to give a final concentration of 1:10000, and used to gate out dead cells during analysis. Cells were incubated with the viability dye on ice in the dark for 30 minutes followed by the addition of $500 \mu \mathrm{L}$ FACS buffer (Appendix 1). Samples were spun at $250 \times \mathrm{g}$ for 5 minutes, the supernatant was removed, and an additional FACS wash was performed before staining with the extracellular CD4 antibody. Cells were stained with a final concentration of $500 \mathrm{ng} / \mathrm{ml}$ anti-human CD4 PE-Cy7 (clone OKT4, Biolegend, CA, USA) in $100 \mu \mathrm{L}$ of FACS buffer for 20 minutes in the dark at RT.

After staining, cells were washed twice with $500 \mu \mathrm{L}$ of FACS buffer and centrifuged at $250 \times \mathrm{g}$ for 5 minutes, leaving a cell suspension of $100 \mu \mathrm{L}$ for intracellular staining. The intracellular staining of the CD4 T cells was performed using the Human Th17/Treg Phenotyping Kit (BD Biosciences, NJ, USA) following manufacturers guidelines. Cells 
were fixed with $500 \mu \mathrm{L}$ of Human FoxP3 Buffer $A$ and incubated for 15 minutes in the dark at RT. The fixative was removed by spinning samples at $500 \mathrm{xg}$ for 5 minutes and the supernatant aspirated carefully to not disturb the buoyant pellet. To permeablize cells, $400 \mu \mathrm{L}$ of FoxP3 Buffer $\mathrm{C}$ was incubated with samples for 30 minutes in the dark at RT. Cells were washed twice with $500 \mu \mathrm{L}$ of FACS buffer and centrifuged at $500 \times \mathrm{g}$ for 5 minutes, leaving a cell suspension of $100 \mu \mathrm{L}$ for intracellular staining. FITClabelled anti-human IFN- $\gamma$ (clone 25723.11, BD Biosciences, NJ, USA) at a final concentration of $7.5 \mu \mathrm{g} / \mathrm{ml}$ as well as $20 \mu \mathrm{L}$ of antibody cocktail containing PE-labelled anti-human IL-17 and Alexa Flour 647-labelled anti-FoxP3 antibodies were added to the appropriate samples. Isotype controls (Appendix 2) and appropriate single stains were included along side phenotyping samples. All samples were stained for $\mathbf{4 0}$ minutes in the dark at RT. At the end of staining, cells were washed twice with $500 \mu \mathrm{L}$ of FACS buffer and spun at $500 \times \mathrm{g}$ for 5 minutes and resuspended in $200 \mu \mathrm{L}$ of FACS buffer for sample acquisition.

\subsubsection{Flow cytometry}

Samples were collected on the FACS Canto II using Diva software (BD Biosciences, NJ, USA). At least 40,000 CD4 positive gated events were collected to allow for a minimum of 20,000 viable CD4 $\mathrm{T}$ cells to be analysed per sample. Anti-mouse Ig, $\mathrm{K} /$ compensation beads ( $\mathrm{BD}$ Biosciences, NJ, USA) were acquired the same time as samples for compensation during analysis. Data was analysed using FlowJo software (Tree star, Ashland, OR, USA). CD4 T cell subgroup populations were determined by the gating strategies as outlined in Figures 8-2 to 8-6 
A

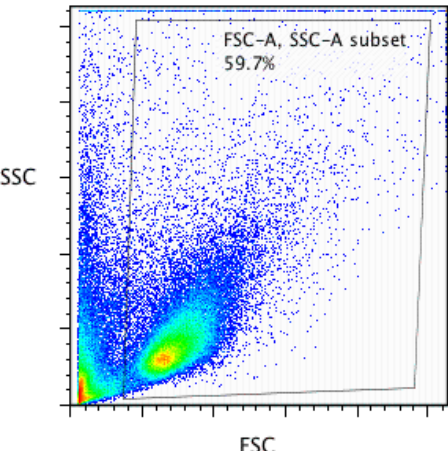

FSC

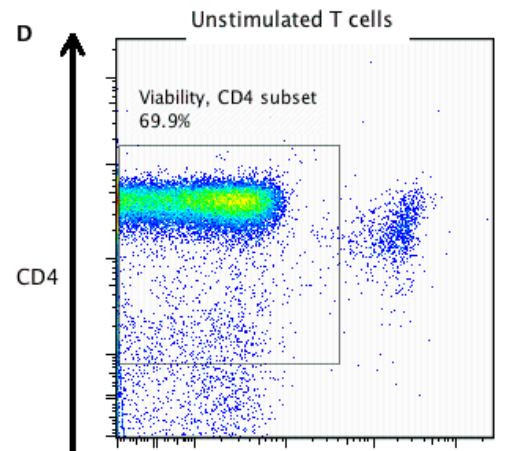

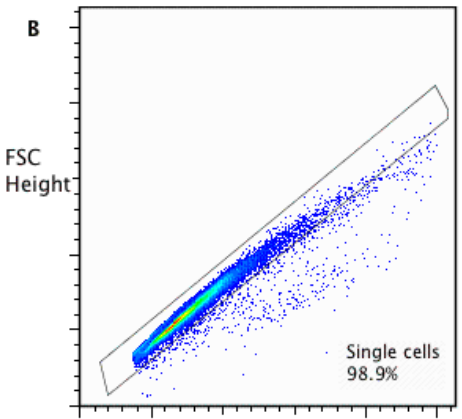

FSC - Area

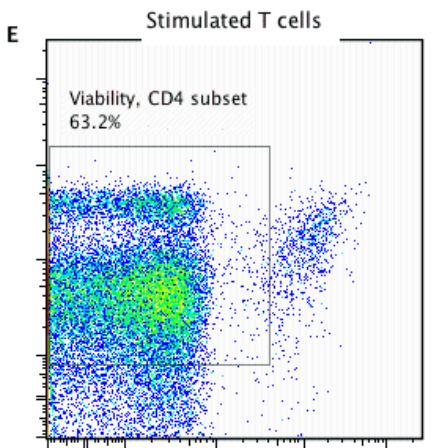

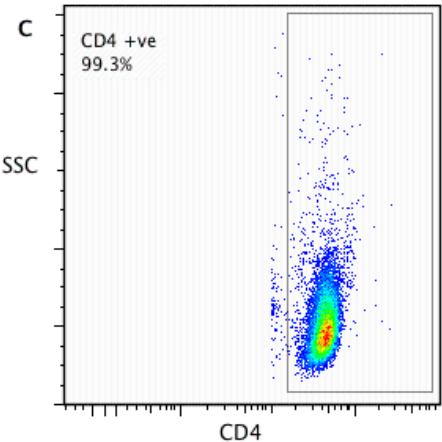

CD4

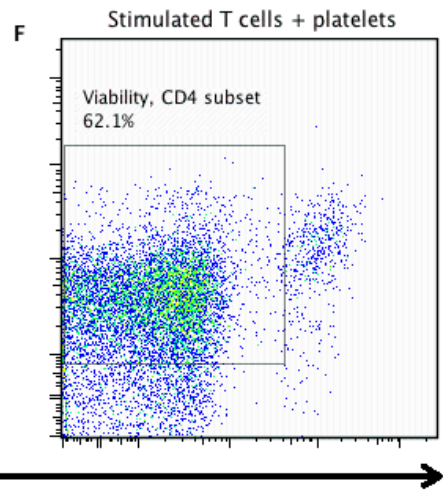

Viability

Figure 8-2 Identification of CD4 T cells

CD4 T cells were identified by excluding debris based on the Forward (FSC) and Side Scatter (SSC) properties (A) and including only single cells (B). With stimulation (E \& F), the expression of CD4 was down regulated and as the majority of cells were CD4 positive in culture (C) as checked after isolation, the high and low CD4 population was included for analysis of proliferation and CD4 T cell sub groups. 


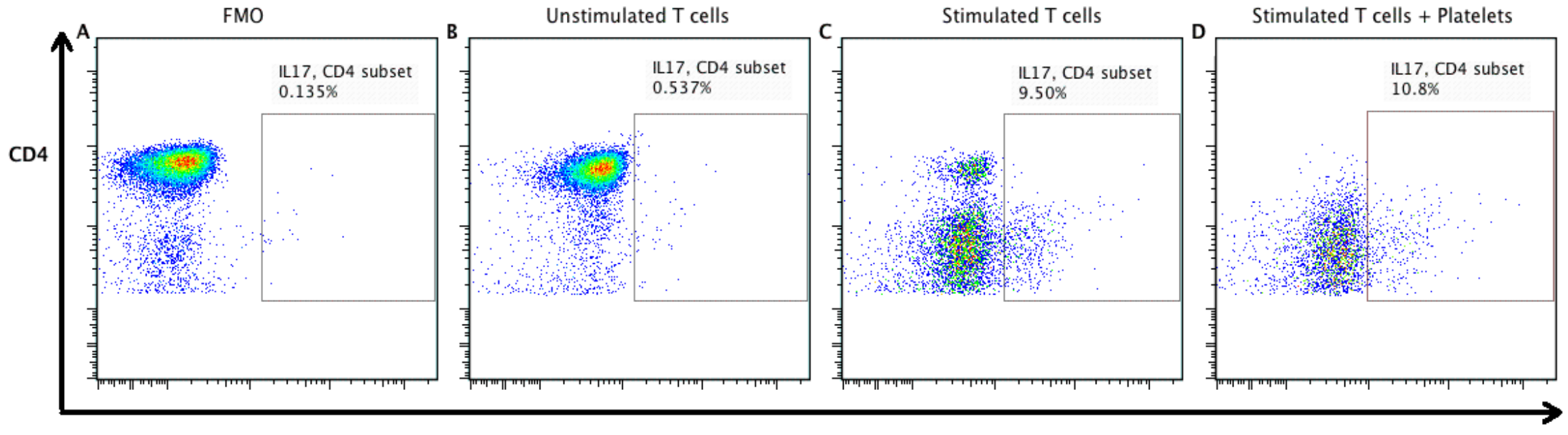

IL-17A

\section{Figure 8-3 Identification of Thelper (Th)1 cells}

T helper 1 (Th1) cells were identified by the expression of IFN-y compared to the isotype control (A). An example of the Th1 population in unstimulated CD4 T cells (B), anti-CD3/CD28 stimulated CD4 T cells (B) and anti-CD3/CD28 stimulated CD4 T cells + platelets (D) are shown. 


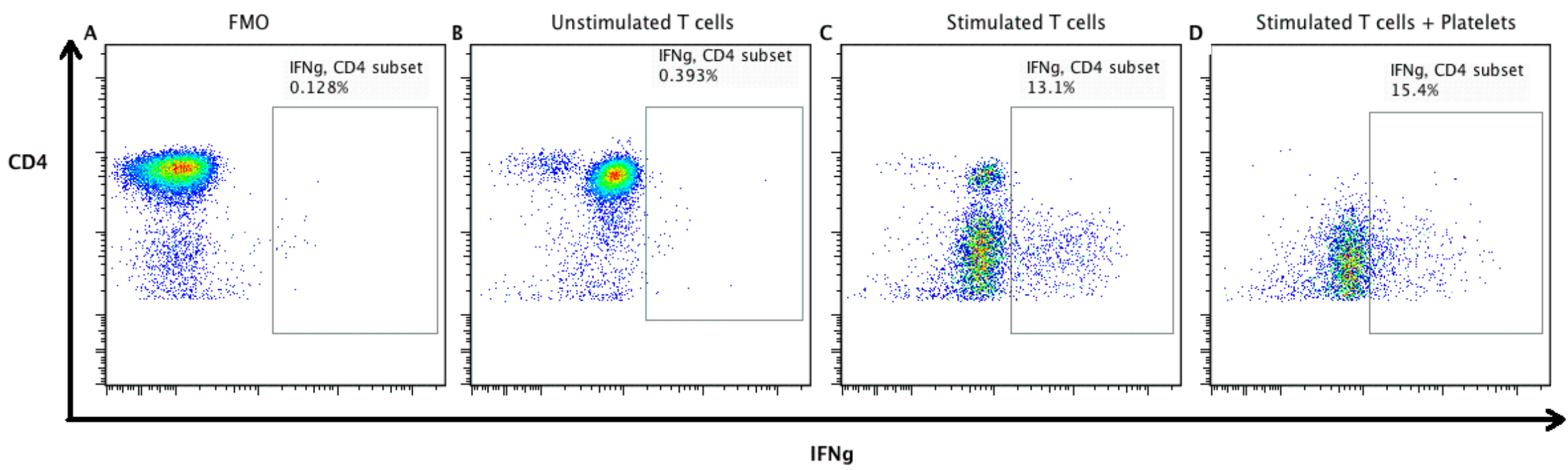

Figure 8-4 Identification of Thelper (Th)17 cells

T helper 17 (Th17) cells were identified by the expression of IL-17A compared to isotype control (A). An example of the Th17 population in unstimulated CD4 T cells (B), anti-CD3/CD28 stimulated CD4 T cells (C) and anti-CD3/CD28 stimulated CD4 T cells plus platelets (D) are shown. 


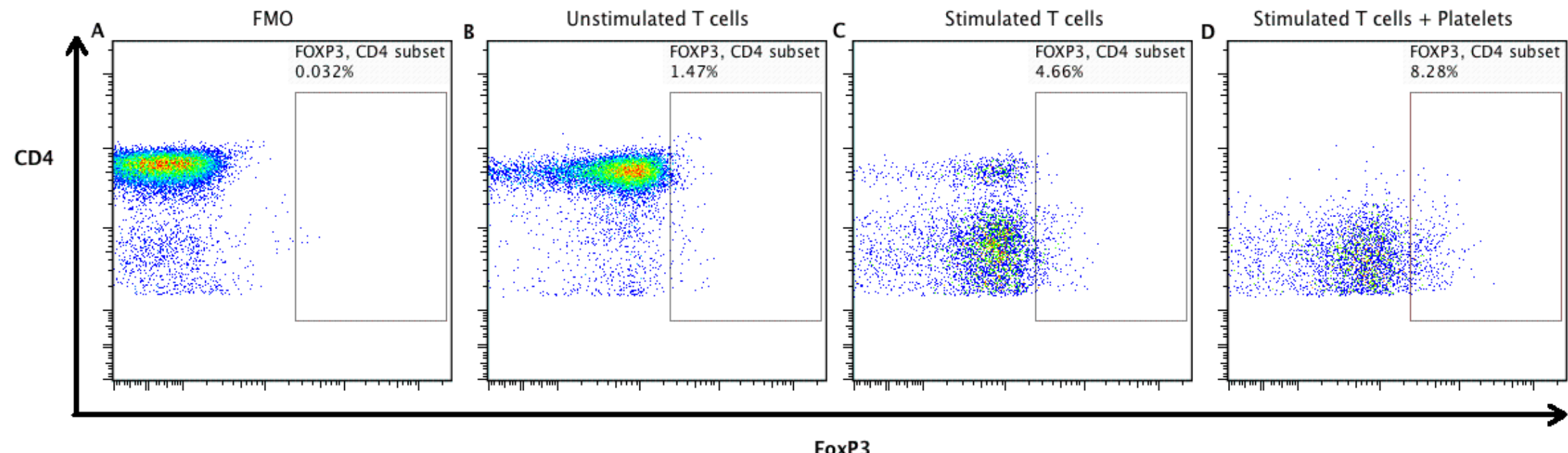

Figure 8-5 Identification of T regulatory (Treg) cells

T regulatory (Treg) cells were identified by the expression of FoxP3 compared to isotype control (A). An example of the Treg population in unstimulated CD4 T cells (B), anti-CD3/CD28 stimulated CD4 T cells (C) and anti-CD3/CD28 stimulated CD4 T cells plus platelets (D) are shown. 


\subsubsection{Quantification of IFN-y production by ELISA}

The production of IFN- $-\gamma$ in the cell culture of unstimulated CD4 T cells, anti-CD3/CD28 stimulated CD4 T cells and stimulated CD4 T cells + platelets was detected using the Legend Max ${ }^{\mathrm{TM}}$ Human IFN- $\gamma$ ELISA kit (Biolegend, CA, USA) following the manufacturers guidelines. Briefly, all reagents were brought to room temperature and the lyophilized Human IFN- $\gamma$ standard was reconstituted in Assay buffer A to give a 20 $\mathrm{ng} / \mathrm{ml}$ standard stock solution. The standard was serially diluted from $1000 \mathrm{pg} / \mathrm{ml}$, to $15.6 \mathrm{pg} / \mathrm{ml}$. The plate was washed and $50 \mu \mathrm{L}$ of sample or standard were added to wells. Unstimulated samples were added undiluted and stimulated and stimulated + platelet samples were diluted 100 fold first and all samples were tested in duplicate. The plate was sealed and incubated at RT for 2 hours while shaking at $200 \mathrm{rpm}$. The plate was washed and incubated in the IFN- $\gamma$ detection antibody solution 1 hour while shaking. Avidin-HRP solution was then added to each well after washing and incubated for 30 minutes. Substrate solution $\mathrm{F}$ was added and incubated for 15 minutes in the dark at RT. The reaction was then stopped and the absorbance was read at $570 \mathrm{~nm}$ and $450 \mathrm{~nm}$ on a Versamax Microplate Reader (Molecular Devices, CA, USA). The OD value at $570 \mathrm{~nm}$ was subtracted from $450 \mathrm{~nm}$ and the level of IFN- $\gamma$ in samples was determined from the standard curve.

\subsubsection{Statistics}

Due to the cross over design of the study, analysis of a potential period effect and treatment-period interaction were performed before comparisons could be done between treatment groups. This analysis was performed following the method outlined by Altman (434). For each parameter to be tested, the difference $\left(d_{i}\right)$ and the average $\left(a_{i}\right)$ for each subject was calculated and averaged in Group A and B. The period effect was tested by a two-sample $t$ test to compare the difference between the periods in the two groups of participants. This test determines whether a group benefits more from receiving the drug first or second. To investigate the treatmentperiod interaction, a two-sample $t$ test comparing the average responses between group $A$ and $B$ was performed. This analysis determines whether there is any carry- 
over of treatment effect from one period to the next and the effectiveness of the wash out period.

Based on the variance of IFN- $\gamma$ in the literature, we calculated a cohort size of 10 patients would give us $80 \%$ power to observe a significant reduction in IFN- $\gamma$ levels following prasugrel of more than $33 \%$. We decided on a group size of 12 to slightly overpower the study

Continuous variables were expressed in mean \pm SEM. Comparisons between stimulation treatments and drug treatments were performed using 1-way ANOVA with post-hoc Bonferroni testing of selected pairs. Statistical analysis and graphs were generated using GraphPad Prism 5 (GraphPad Software Inc., CA, USA).

\subsection{Results}

\subsubsection{Period effect and treatment-period interaction analysis}

To assess how platelet interactions with $\mathrm{T}$ cells could shape $\mathrm{T}$ cell phenotype and subset differentiation, a double blind, placebo controlled, cross over trial was performed on 12 healthy volunteers with a median age of $31(21-45)$. Due to the cross over trial design, there was a possibility of period effects or treatment-period interactions. However, we found no statistically significant period effect or treatmentperiod interaction between group $A$ and group $B$. For example, the difference in $T$ helper 1 cells for the drug and placebo period are calculated for each individual in group A (Table 8-1) and group B (Table 8-2). There was no difference between $đ_{1}$ and $đ_{2}(p=0.34)$ and therefore no period effect. There was also no significant difference between $\bar{a}_{1}$ and $\bar{a}_{2}(p=0.25)$, which indicates the wash out period was sufficient and there was no treatment-period interaction. This analysis was repeated for every parameter and no period effect or treatment-period interaction was found. Therefore comparisons between baseline and treatments and between the two different treatments could be undertaken without adjustment. 
Table 8-1 Group A: Drug (prasugrel) followed by placebo $\mathrm{n}=6$

\begin{tabular}{|l|l|l|l|l|}
\hline & $\begin{array}{l}\text { Period 1 } \\
\text { Drug }\end{array}$ & $\begin{array}{l}\text { Period 2 } \\
\text { Placebo }\end{array}$ & $(1)-(2)$ & $(1)+(2) / 2$ \\
\hline 1 & $-499 \%$ & $-42 \%$ & -4.57 & -2.71 \\
\hline & $-72 \%$ & $27 \%$ & -0.99 & -0.23 \\
\hline & $-92 \%$ & $-96 \%$ & 0.04 & -0.94 \\
\hline & $-104 \%$ & $43 \%$ & -1.47 & -0.30 \\
\hline & $-29 \%$ & $23 \%$ & -0.51 & -0.03 \\
\hline Mean & $-44 \%$ & $22 \%$ & -0.66 & -0.11 \\
\hline SD & $-140 \%$ & $-4 \%$ & $-136 \%\left(\mathbb{đ}_{1}\right)$ & $-72 \%\left(\overline{\mathrm{a}}_{1}\right)$ \\
\hline
\end{tabular}

Table 8-2 Group B: Placebo followed by drug (prasugrel) $n=6$

\begin{tabular}{|l|l|l|l|l|}
\hline & $\begin{array}{l}\text { Period 1 } \\
\text { Placebo }\end{array}$ & $\begin{array}{l}\text { Period 2 } \\
\text { Drug }\end{array}$ & $(1)-(2)$ & $(1)+(2) / 2$ \\
\hline & $84 \%$ & $-87 \%$ & 1.72 & -0.01 \\
\hline & $-110 \%$ & $-155 \%$ & 0.44 & -1.32 \\
\hline & $-40 \%$ & $-45 \%$ & 0.05 & -0.42 \\
\hline & $-67 \%$ & $-114 \%$ & 1.81 & -0.23 \\
\hline & $-15 \%$ & $-113 \%$ & 0.98 & -0.64 \\
\hline Mean & $-3 \%$ & $-115 \%$ & 1.12 & -0.59 \\
\hline SD & $71 \%$ & $-105 \%$ & $102 \%\left(\mathbb{d}_{2}\right)$ & $-54 \%\left(\overline{\mathrm{a}}_{2}\right)$ \\
\hline
\end{tabular}

${ }^{1}$ Difference in T helper 1 cells for the drug and placebo period are calculated for each individual and are expressed as \% change between baseline and measurement following drug treatment. 


\subsubsection{Platelet function following placebo and prasugrel therapy}

To assess whether the treatments were having the desired impact on platelet function, platelet reactivity was measured with the Multiplate before and after each therapy (Figure 8-6). Treatment with placebo resulted in no change in platelet function between baseline and placebo treatment. However, following prasugrel therapy, all participants had a marked reduction in platelet reactivity (86.8 AU $\pm 7.7 \mathrm{AU}$ vs. 27.3 $A U \pm 5.2 \mathrm{AU}, \mathrm{p}<0.0001$ ). This finding confirms that prasugrel was having a significant and expected antiplatelet effect in the study participants.

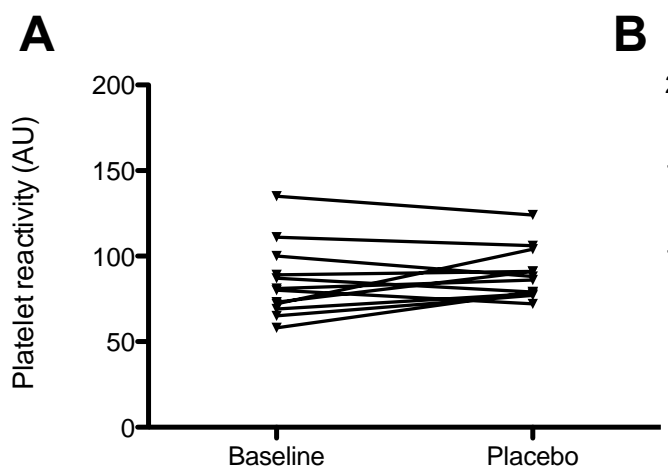

B

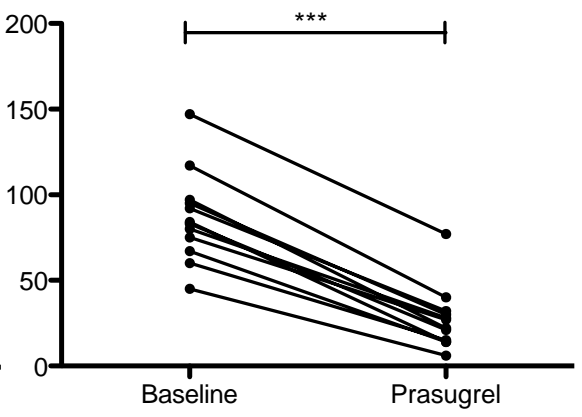

Figure 8-6 Marked reduction in platelet function following prasugrel therapy

Platelet function $(\mathrm{AU})$ as measured by Multiplate in whole blood, before and after placebo therapy (A) and prasugrel therapy (B). Each bar represents a participant's response before and after each treatment $n=12 .{ }^{* * *} \mathrm{p}<0.0001$ by paired Student's $t$ test.

\subsubsection{IFN-y production and CD4 T cell differentiation at baseline}

To investigate the impact of platelets on CD4 $\mathrm{T}$ cell IFN- $\gamma$ production and $\mathrm{T}$ cell differentiation, IFN- $\gamma$ production and the Th1, Th17 or Treg phenotype was assessed on purified, anti-CD3/CD28 stimulated CD4 T cells (isolated at baseline) co-cultured with or without platelets. As expected, unstimulated cultures exhibited a low level of IFN- $\gamma$ production and only a small proportion of CD4 T cells expressing the Th1, Th17 or Treg phenotype (Figure 8-7). With the addition of anti-CD3/CD28 T cell stimulation there was a significant increase in all measured parameters. IFN- $\gamma$ production significantly increased to an average production of $258 \mathrm{ng} / \mathrm{ml} \pm 60 \mathrm{ng} / \mathrm{ml}$ from undetectable in unstimulated cultures ( $p<0.01$, Figure 8-7A). The proportion of CD4 T cells positive for the Th1 phenotype (i.e. IFN- $\gamma$ production) significantly increased to 
$7.8 \% \pm 0.8 \%$ with stimulation of $\mathrm{T}$ cells compared to $0.5 \% \pm 0.05 \%$ in unstimulated controls ( $p<0.001$, Figure 8-7B). The proportion of CD4 T cells positive for the Th17 phenotype (i.e. IL-17 production) significantly increased stimulation of T cells to $3.4 \%$ $\pm 0.4 \%$ of CD4 T cells compared to $0.8 \% \pm 0.2 \%$ in unstimulated ( $p<0.001$, Figure $8-7 C$ ). The proportion of CD4 T cells positive for the Treg phenotype (i.e. FoxP3 expression) significantly increased with stimulation of T cells, accounting for $11.2 \% \pm 1.3 \%$ of CD4 T cells compared to $1.4 \% \pm 0.4 \%$ in unstimulated cultures $(p<0.01$, Figure $8-7 D)$.

In co-cultures of anti-CD3/CD28 stimulated T cells and autologous platelets there was further increase in the IFN- $\gamma$ production and Th1 and Th17 subgroups. Co-culture resulted in a significant enhancement of IFN- $\gamma$ production with an average production of $454 \mathrm{ng} / \mathrm{ml} \pm 78 \mathrm{ng} / \mathrm{ml}(\mathrm{p}=0.019$, Figure $8-7 \mathrm{~A})$. The addition of platelets further enhanced the Th1 population at baseline to $10.8 \% \pm 0.8 \%(p=0.01$, Figure $8-7 \mathrm{~B})$. Similarly, co-culture resulted in the increase of the Th17 population to $5.4 \% \pm 0.7 \%$ (p $=0.01$, Figure $8-7 \mathrm{C})$. In contrast, the addition of platelets did not significantly alter the Treg subpopulation compared to stimulated alone $(p=0.07$, Figure 8-7D). These results indicate that stimulation with anti-CD3/CD28 drives the production of IFN- $\gamma$ and promotes the differentiation of Th1, Th17 and Treg phenotypes and that the presence of platelets further enhances IFN- $\gamma$ as well as Th1 and Th17 differentiation. 
A

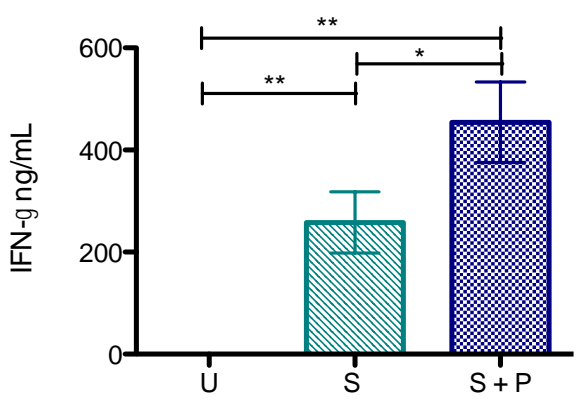

C

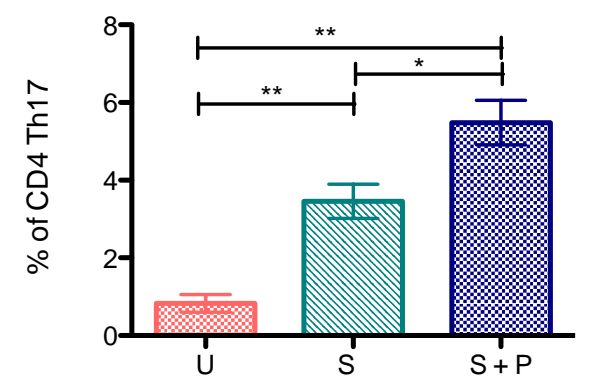

B

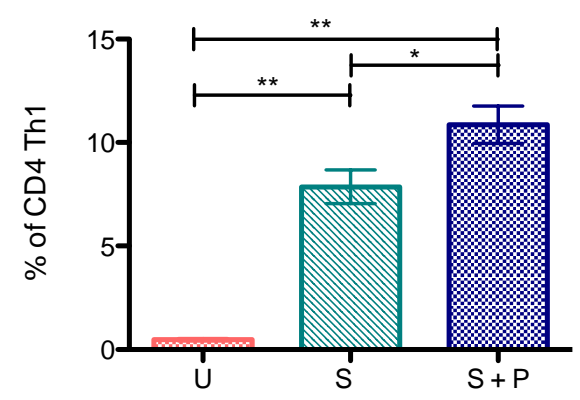

D

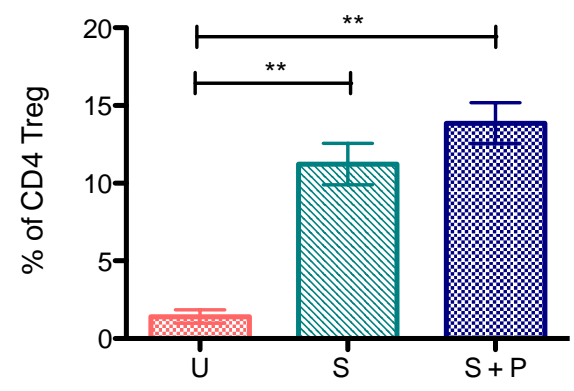

Figure 8-7 The addition of platelets to anti-CD3/CD28 stimulated CD4 T cells enhances the production of IFN- and Th1 and Th17 differentiation.

Isolated CD4 T cells at baseline were cultured without stimulation (U), with anti-CD3/CD28 stimulation (S) and stimulation plus autologous platelets $(S+P)$. IFN- $\gamma$ production was measured in the supernatant of cultures with ELISA and the CD4 T cell subsets were measured by intracellular cytokine staining for IFN-y (Th1), IL-17A (Th17) and FoxP3 (Treg) using flow cytometry. IFN-y production is expressed in $\mathrm{ng} / \mathrm{ml}(\mathrm{A})$ and the subsets are expressed as the proportion of CD4 T cells expressing the Th1 phenotype (B), Th17 phenotype (C) and Treg phenotype (D) in each culture condition. Data is displayed as mean \pm SEM for every measurement. The data represents all 12 patients. One way ANOVA was performed for each parameter and was found to be significant $(p<0.05)$ allowing paired Student's $t$ tests to be performed $\left({ }^{*} p<0.05,{ }^{* *} p<0.01\right)$. 


\subsubsection{The effect of prasugrel on IFN-y production and CD4 T cell differentiation}

To determine if treatment with a placebo alone altered IFN- $\gamma$ production and the CD4 $\mathrm{T}$ cell subset differentiation from baseline measurements, these parameters were measured following placebo treatment in all participants and compared to the baseline values. No significant change was seen in IFN- $\gamma$ production (Figure 8-8) or the CD4 T cell subset differentiation (see Appendix 3) compared to baseline. Additionally, there was no significant change for any of the parameters in unstimulated cultures from the prasugrel group compared to placebo or baseline. Therefore, given that baseline and placebo responses were the same and the unstimulated cultures were not affected by any treatment, the stimulated CD $4 \mathrm{~T}$ cell responses following prasugrel treatment were directly compared to the placebo treatment to more specifically assess the effect of prasugrel treatment on platelet-T cell interactions.

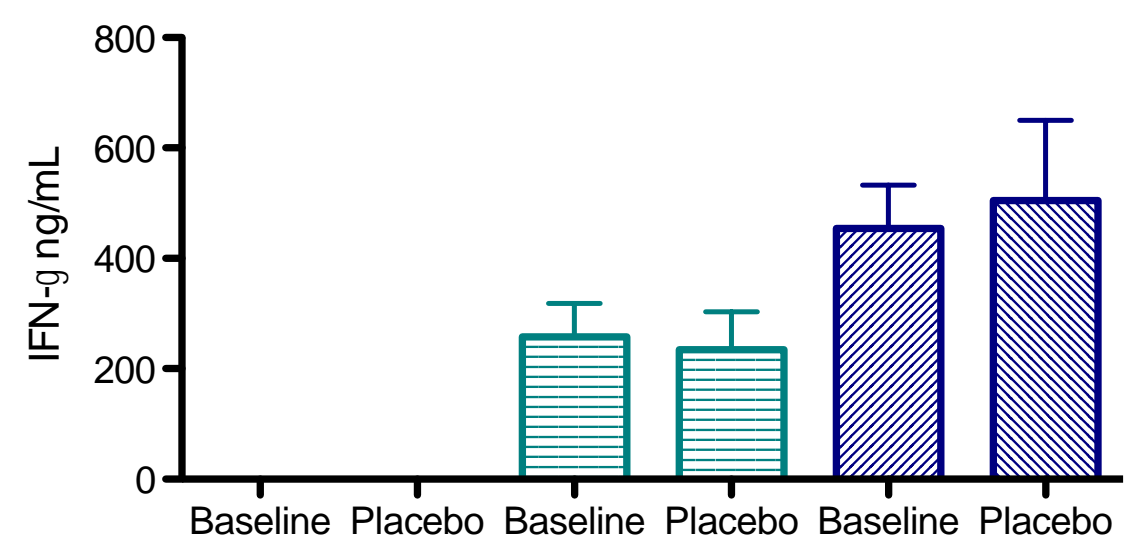

U

S

$S+P$

\section{Figure 8-8 No difference in isolated CD4 T cell IFN-y production following placebo therapy compared to baseline in all culture conditions}

Isolated CD4 T cells at baseline and after placebo therapy were cultured without stimulation $(\mathrm{U})$, with anti-CD3/CD28 stimulation (S) and stimulation plus autologous platelets (S + P). IFNY production was measured in the supernatant of cultures with ELISA and expressed in $\mathrm{ng} / \mathrm{ml}$. Data is displayed as mean \pm SEM for every measurement. The data represents all 12 patients. One way ANOVA was performed for each parameter and was found to be significant $(p<0.05)$ allowing paired $\mathrm{T}$ tests to be performed of culture conditions.

Focusing on this comparison, there were several significant changes in the co-culture effects following prasugrel treatment. The level of IFN- $\gamma$ in stimulated T cell-platelet cultures was significantly lower at $89 \mathrm{ng} / \mathrm{ml} \pm 17 \mathrm{ng} / \mathrm{ml}$ compared to co-culture 
samples following placebo treatment $(\mathrm{p}<0.01$, Figure 8-9A) and the stimulatory effect of platelets on T cells was abolished. This change was also evident in the Th1 subgroup with a significant decrease in the proportion of CD4 T cells that was Th1 positive compared to co-cultures in the placebo group $(5.9 \% \pm 0.8 \%$ vs $10.2 \% \pm 1.1 \%, p<0.01$, Figure 8-9B). Furthermore, the Th17 response was not enhanced with the addition of platelets to stimulated $\mathrm{T}$ cell cultures following prasugrel therapy resulting in comparable levels to when T cells were stimulated alone $(2.7 \% \pm 0.4 \%$ vs. $2.7 \% \pm 0.2 \%$, Figure 8-9C). As seen in the placebo group, the addition of platelets to stimulated $T$ cells did not alter the proportion of Tregs following prasugrel therapy (Figure 8-9D). Taken together these findings suggest that following prasugrel treatment, the enhancement of the pro-inflammatory CD4 T cell phenotype by platelets following anti-CD3/CD28 stimulation is abolished. 

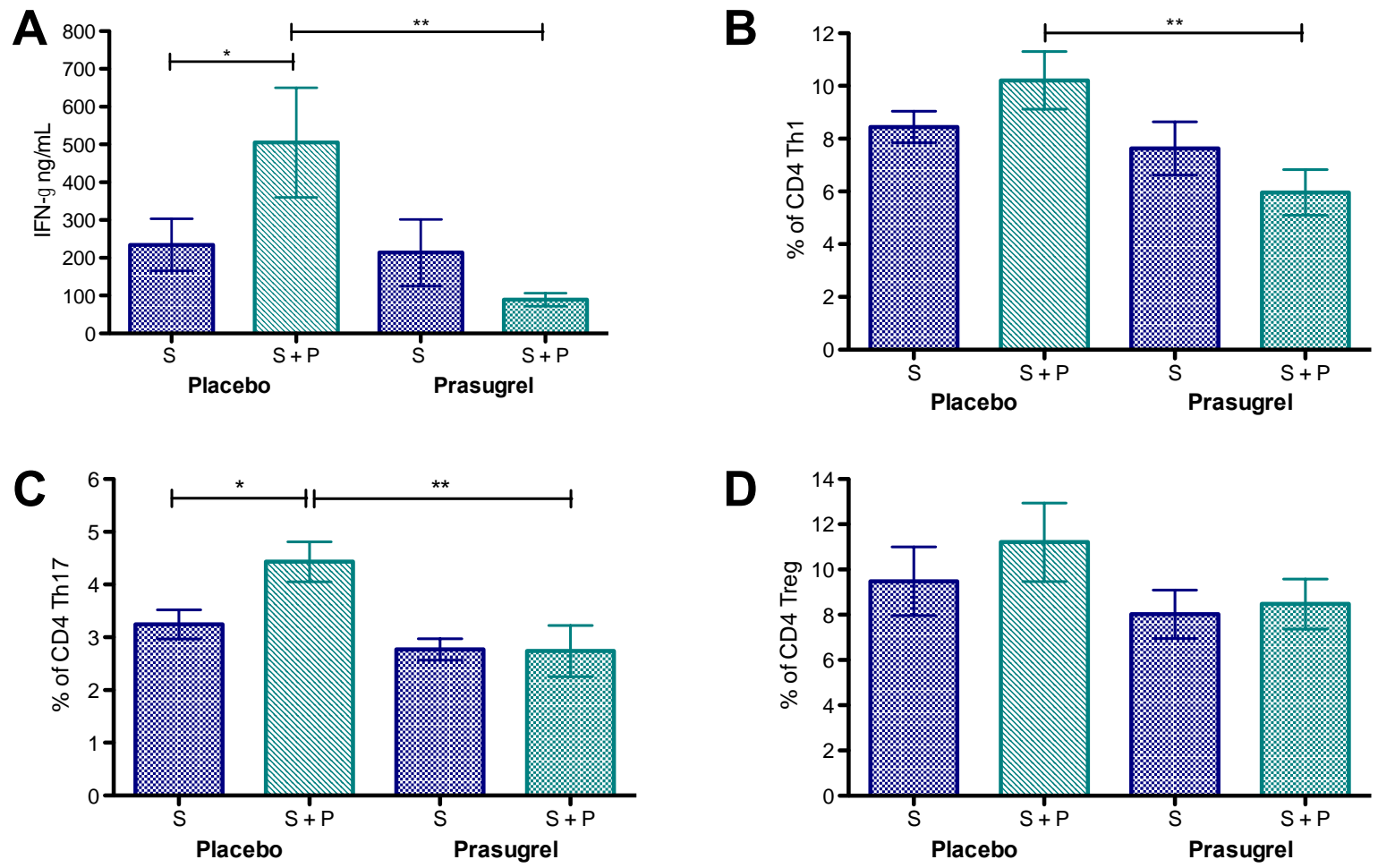

\section{Figure 8-9 Prasugrel therapy abolishes the enhancement of isolated CD4 T cell IFN-y production and Th1 and Th17 differentiation by platelets}

Isolated CD4 T cells following placebo and prasugrel treatment were cultured with antiCD3/CD28 stimulation (S) and stimulation plus autologous platelets ( $+\mathrm{P})$. IFN-y production was measured in the supernatant of cultures with ELISA and the CD4 T cell subsets were measured by intracellular cytokine staining for IFN-Y (Th1), IL-17A (Th17) and FoxP3 (Treg) using flow cytometry. IFN-y production is expressed in $\mathrm{ng} / \mathrm{ml}(\mathrm{A})$ and the subsets are expressed as the proportion of CD4 T cells expressing the Th1 phenotype (B), Th17 phenotype (C) and Treg phenotype (D) in each culture condition. Data is displayed as mean \pm SEM for every measurement. The data represents all 12 patients. One way ANOVA was performed for each parameter and was found to be significant $(p<0.05)$ allowing paired Student's tests to be performed ( $\left.{ }^{*} p<0.05,{ }^{* *} p<0.01\right)$. 


\subsection{Discussion}

This study investigated the impact of platelet-T cell interactions on CD4 T cell IFN- $\gamma$ production and Th subset differentiation. Stimulation of T cells with the known T cell stimulant, anti-CD3/CD28, induced a significant production of IFN- $\gamma$ and an increase in Th1, Th17 and Treg subsets confirming that our experimental model was functional. The addition of platelets to stimulated T cells caused a further enhancement of IFN- $\gamma$ production and greater proportion of Th1 and Th17 subsets. However, Tregs were less affected by platelet co-culture. The effect of inhibiting platelet activation in vivo with prasugrel on $\mathrm{T}$ cell responses was also determined and indicated that prasugrel treatment abolished the platelets' ability to augment IFN- $\gamma$ production and Th1 and Th17 differentiation.

Platelet function testing confirmed prasugrel induced a marked inhibition in platelet activity in all subjects. However, prasugrel administration did not affect IFN- $\gamma$ production or $\mathrm{T}$ cell differentiation in unstimulated or anti-CD3/CD28 stimulated $T$ cell cultures in the absence of platelets. These two observations along with our findings from co-culture stimulations, suggest that platelets are potent regulators of CD4 T cell differentiation and cytokine production and that the impact of prasugrel on cytokine production and differentiation is due to the drug's effects on platelet function.

The addition of platelets to stimulated T cells resulted in a $340 \%$ increase in IFN- $\gamma$ levels in the supernatant of cell cultures and a $40 \%$ increase in Th1 cell populations compared to stimulated alone. Th1 cells are the primary subset of CD4 T cells found in atherosclerotic lesions and secrete the pro-inflammatory cytokines, IFN- $\gamma$, IL-2 and TNF- $\alpha$, which can activate other lesional cells to promote inflammation and augment lesion development (433). From various in vitro experimental settings, platelets and their derived mediators have shown to regulate Th1 differentiation and function. The platelet chemokines RANTES and MCP-1 have been demonstrated to enhance the production of IL-2, an important cytokine for T cell survival, from Th1 cells stimulated with anti-CD3/CD28, (424), and the neutralization of RANTES inhibited IL-2 and TNF- $\alpha$ production in CD4 T cell-platelet co-culture (434). Another platelet derived 
chemokine, PF4 has demonstrated regulatory effects on CD4 T cells, although not always stimulatory depending on the environment. With cultured total T cells, PF4 inhibited IL-2 and IFN- $\gamma$ production in response to both antigenic and polyclonal stimulation (435). However, a recent study demonstrated PF4 enhanced Th1 differentiation and cytokine production when CD4 T cells were cultured with autologous platelets, which the authors attributed to Th1 effector and memory cell stimulation (434).

Other platelet-related inflammatory mediators, such as thromboxane $A_{2}$, and plateletactivating factor (PAF) also display Th1 cell-regulatory effects. Activated platelets produce a significant amount of $\operatorname{TxA}_{2}$, which inhibits Th1 proliferation and cytokine production (436). Activated T cells express PAF receptor (437), and PAF can enhance Th1 IFN- $\gamma$ production of cultured T cells $(437,438)$. Finally, IL-1 $\beta$ a known potent promoter of Th1 differentiation from naïve CD4 T cells and this cytokine can be synthesized in platelets upon activation and deposited into fibrin nests of thrombi (439). Hence, a thrombus may serve as a reservoir of highly concentrated plateletderived cytokines and mediators that enhance platelet-dependent regulation of T cells in thrombi. Many of the studies have investigated Th1 differentiation and cytokine production with the addition of an isolated platelet derived mediator, but this study is the first to investigate the impact of platelets on Th1 differentiation and IFN- $\gamma$ production ex vivo with the involvement of all platelet-derived mediators. Our findings suggest that overall platelets have a stimulatory effect on cytokine production and Th1 differentiation.

Furthermore, this is the first study to ascertain the impact of inhibiting platelet-T cell interactions with a $\mathrm{P} 2 \mathrm{Y} 12$ inhibitor in vivo, on $\mathrm{T}$ cell differentiation, cytokine production and proliferation. After prasugrel administration in participants, the addition of platelets to $T$ cell cultures dramatically reduced the production of IFN- $\gamma$ and Th1 differentiation to levels comparable to stimulated alone. Prasugrel eliminated the platelets' stimulatory affects on Th1 cells. In animal models, it has been consistently demonstrated that the inhibition of Th1 cell differentiation or deficiency of Th1 cytokine receptors markedly reduces lesion formation $(440,441)$. As IFN- $\gamma$ is an 
important activator of monocytes and macrophages to become highly inflammatory, which are key inflammatory cells in atherosclerotic lesions, inhibiting this cytokine can reduce inflammation. Therefore, antiplatelet therapy may be providing therapeutic benefits by indirectly modulating Th1 activity and reducing pro-inflammatory cytokine production.

Co-cultures of stimulated CD4 T cells and platelets also promoted a significant enhancement in Th17 differentiation. Th17 cells are a recently recognized CD4 T cell lineage in atherosclerotic lesions and previously have been acknowledged for their critical role in host defense and autoimmunity. Th17 cells secrete its signature cytokine IL-17, which acts on other cells to produce pro-inflammatory cytokines and chemokines and is the clear pro-inflammatory role of Th17 cells in autoimmune disease (444). The impact of Th17 cells in atherosclerosis however remains controversial. Patients with ACS have more circulating Th17 cells and higher plasma levels of IL-17, IL-6 and IL-23 (20) and mouse models of IL-17 deficiency show a reduction in atherosclerotic lesions (443). In contrast to these studies, which suggest the Th17 phenotype is pathogenic, other studies have found increased Th17 differentiation and IL-17 in human atherosclerotic plaques are associated with a stable plaque phenotype (22).

Despite this controversy, several platelet-derived mediators are thought to regulate Th17 differentiation and cytokine production. Platelets have abundant stores of TGF $\beta$, which is released and acts with IL- 6 and IL-21 to promote Th17 differentiation. TGF $\beta$ promotes Th17 differentiation in the presence of IL- 6 whereas TGF $\beta$ alone drives Treg differentiation (425). As already mentioned, platelets can synthesise IL-1 $\beta$ and the release of this causes Th17 polarisation through inducing RORyt expression, a key transcription factor in Th17 differentiation (444). This cytokine is also critical in maintaining the steady status of Th17 cells and IL-6 acts to enhance IL-1 $\beta$ activity (444). Moreover, platelet activation leads to PAF synthesis and release, which can promote Th17 differentiation and cytokine production (445). 
Prasugrel administration abolished the enhancement of Th17 differentiation by platelets in co-culture with T cells and produced similar Th17 levels to stimulated alone. Our results would suggest that platelets have a potent affect on Th17 differentiation and antiplatelet treatment can inhibit this stimulus. However, it is unknown what affect and to what extent this inhibition would impact on atherosclerosis as the role of Th17 cells in atherogenesis remains controversial.

CD4 T regulatory (Treg) cells are key anti-inflammatory cells in the immune system and operate to govern immunological tolerance and the terminate immune responses. With anti-CD3/CD28 stimulation, a significant increase in Treg differentiation was achieved at all time points. However, the addition of platelets did not significantly enhance the Treg response compared to stimulated alone. Other studies have found platelets can have a marked effect on Treg differentiation via platelets abundant stores of TGF $\beta$ (434). TGF $\beta$ is essential for Treg differentiation and high local concentrations selectively promote this CD4 phenotype (425). However, neutralization of this growth factor did not prevent platelet-enhanced Treg differentiation, indicating there are other platelet-derived factors that may be impacting on Treg differentiation. Platelet factor 4 (PF4) and IL-1 $\beta$ have both been indicated in in vitro models to enhance anti-CD3/CD28-stimulated Treg differentiation (446). However, the influence of these mediators on Treg development can be augmented by the presence of IL-2 and by the level of TGF $\beta$, indicating the regulation of Treg in vivo is likely complex. In our present study, the net impact of platelets on Treg differentiation appears to be small and variable between healthy individuals.

Not surprisingly, platelet inhibition with prasugrel did not reduce Treg differentiation significantly. The presence of Treg at atherosclerotic lesions can act to suppress immune responses in vulnerable plaques. Many strategies have been investigated to increase Treg activity in atherosclerosis with little success. Potentially the inhibition of platelets through the P2Y12 pathway can impact on Th1 and Th17 cells without altering Treg differentiation significantly, although further investigation would be needed to confirm this. 
Overall, as this study has demonstrated, platelets are not solely anucleate aggregating bodies driving thrombosis but can also communicate with cells including CD4 T cells to influence differentiation and cytokine production. While this study has primarily considered the role of platelet-T cell interactions in atherosclerosis, our findings have implications that extend beyond atherosclerosis and which may suggest a role for platelet-lymphocyte interactions in other diseases such as but not limited to; sepsis, rheumatoid arthritis, cancer, asthma and cystic fibrosis to $(75,449)$. Thus the novel finding that prasugrel can abolish the ability of the platelet to augment CD4 T cell stimulation highlights the potential use of antiplatelet therapy in other inflammatory conditions.

\subsection{Limitations}

One of the limitations of this study was the size of the study population and therefore the statistical power to detect subtle changes in the various parameters. As originally anticipated, with 12 participants we had the power to detect a $>30 \%$ reduction in IFN$\gamma$ levels following prasugrel therapy compared to placebo. However, with higher numbers of participants, we would have had the power to detect more subtle changes, such as the change in Treg differentiation and the interesting decrease in IFN- $\gamma$ levels in stimulated samples following prasugrel treatment. Nevertheless, with 12 participants we were able to detect significant and interesting effects of platelets on IFN- - production and Th1 and Th17 subsets.

Additionally, we looked at a limited number of CD4 T cell subsets and markers of cell function. It would have been interesting to look at Th2 subsets and other important populations of $T$ cell such as natural killer T cells (i.e. NKT). However, from the literature it has been shown that Th1, Th17 and Treg subsets have a significant influence in atherosclerosis and therefore the impact of platelets on these cells was more likely to offer the most relevant results for this thesis. Finally, IFN- $\gamma$ was the only cytokine to be quantified in culture supernatants due to the cost of ELISA kits, but in the future, it would be vaulable to measure other cytokines, both inflammatory and regulatory, to provide a fuller picture of the effect of platelets on CD4 T cell effector functions. 


\subsection{Conclusion}

Our findings suggest that platelets are potent regulators of CD4 T cell differentiation and IFN- $\gamma$ production. The addition of platelets to stimulated T cells enhanced the production of IFN- $\gamma$ and the differentiation of Th1 and Th17 subsets compared to single cultures of anti-CD3/CD28 stimulated $T$ cells in healthy individuals. The administration of prasugrel abolished the platelets ability to boost Th1 and Th17 differentiation and the production of IFN- $\gamma$ suggesting that antiplatelet therapy may provide therapeutic benefits through the inhibition of the platelets' thrombotic ability and an indirect modulation of the immune response. 
- Chapter 9 - Summary and future directions 


\subsection{Summary}

In this thesis, the first aim of investigation was to understand the reproducibility and methodological factors that influenced the Multiplate's measurement of residual platelet reactivity. The Multiplate system had a moderate CV at $10-11 \%$ and the preanalytical factors of time delay to testing and anticoagulant blood tubes, affect the assessment of platelet aggregation and need to be standardised for all measurements. Using the Multiplate to measure residual platelet reactivity in a NZ ACS population treated with aspirin and clopidogrel, the study found HOTPR was common and occurred more frequently in Maori and Pacific Islanders. Patients with diabetes or receiving low clopidogrel doses also had higher rates of HOTPR. The use of prasugrel in patients with HOTPR resulted in a marked reduction in residual platelet reactivity for all patients.

Further investigation of the relationship between genetic and clinical factors with residual platelet reactivity was conducted to identify independent predictors of this phenotype. This study revealed that diabetes, low clopidogrel dose and high platelet count were all strong independent predictors of high residual platelet reactivity. Genetic variation in the CYP2C19 gene only moderately contributed to the observed variation in the clopidogrel response. In this study there were no clinical or genetic factors that were driving a low residual platelet reactivity response to clopidogrel.

In addition to understanding the drivers of the on treatment phenotype, the relationship between phenotype, genotype and predictors of phenotype with MACE and bleeding events were also examined following an ACS. It was demonstrated that both phenotype and diabetes were independent predictors of MACE and genotype was not a significant driver of risk. Bleeding was low in the cohort and was not predicted by phenotype, genotype or clinical factors.

Another population at high risk of thrombotic complications are patients undergoing major vascular surgery. The aim was to investigate whether biomarkers including platelet reactivity and other established cardiac risk markers had utility at predicting 
thrombotic and bleeding outcomes in this population. Baseline elevated hs-TnT was the most predictive biomarker and was significantly associated with an increased risk of ischaemia following surgery. Residual platelet reactivity did not predict ischaemia or bleeding in this population.

The interactions of platelets with $\mathrm{T}$ lymphocytes in patients with atherosclerotic disease and healthy volunteers were also investigated. The first exploratory study revealed a higher level of platelet-T cell conjugates in vascular patients compared to healthy controls. An interesting observation was found in the CD4 T cell population with a switch in the activation phenotype to predominantly an effector phenotype in vascular patients, which were not observed in healthy controls. Further examination of platelet interactions with CD4 T cells in healthy volunteers; suggest platelets are potent regulators of the CD4 T cell differentiation and cytokine production and this stimulation can be abolished with the administration of prasugrel.

\subsection{Limitations}

Across the thesis there have been certain limitations that may have influenced our findings. A large majority of the studies were observational in nature. Although we wanted to look at residual platelet reactivity and biomarkers in a real world setting so our results had wider clinical applicability, this limited our ability to control for differences in medication and patient management, which may have introduced unknown variance. We were limited to one measure of platelet function using only the Multiplate assay and the use of another assay such as VerifyNow may have allowed for interesting comparisons in the ability for an assay to identify patients at risk of MACE. However, the VerifyNow has a high test cost of $\$ 70 /$ patient and the addition of this test in all patients was not feasible. As discussed in chapter 2, we felt the low consumable cost and moderate coefficient of variance achieved with the Multiplate was the best option to measure residual platelet reactivity. Finally, our study populations were of a moderate size and in some instances, reduced our power to investigate relationships between clinical factors, platelet reactivity and outcome. 
However, with the time constraints of the thesis and factoring in the time for follow up for two of our cohorts, recruiting larger patient cohorts were not possible.

\subsection{Clinical implications and future directions}

The findings from this thesis have several clinical implications and raise further avenues of investigation. Some of the observations in our studies may further explain the failure of clinical trials to demonstrate a benefit from personalised antiplatelet therapy based on platelet function testing. As we have shown in this study, the method used to measure residual platelet reactivity needs to be standardised to reduce variance in the test. Reported platelet function trials have primarily used the VerifyNow assay to tailor therapy and a protocol of 10 minutes to 4 hours time delay from blood sampling to measurement is recommended (454). Although we have not specifically tested the effect of time delay on the VerifyNow assay, our results suggest that time has a significant impact on the precision of an assay and this added variance may have reduced the ability to discern which patients would benefit from tailored therapy from a single cut point.

There is sufficient evidence from multiple studies including ours, that high residual platelet reactivity is a powerful independent predictor of MACE in ACS patients. With the increasing use of new potent P2Y12 inhibitors and the availability of generic clopidogrel, it will be left to the decision of the attending physician which drug to choose for the individual patient by balancing the risk of thrombotic and bleeding events. The potential clinical benefit and cost difference from personalised therapy suggest further evaluation of platelet reactivity testing is attractive. However, our study highlighted that platelet reactivity alone does not predict a patient's thrombotic risk. We also identified diabetes as a significant predictor of MACE, and it is likely that other clinical factors will also influence risk. With a combined risk factor strategy including platelet function testing and clinical factors, the ability to identify patients at high risk of thrombotic events may be improved beyond a single biomarker strategy. Using a risk model such as this to personalise antiplatelet therapy has not been investigated and larger outcome studies in ACS patients are needed to ascertain 
the key clinical factors that predict risk and whether such a strategy would improve patient outcomes.

Our study also supports the notion that the loss of function CYP2C19*2 allele is only having a minor impact on an individual's response to clopidogrel $(228,309)$. Moreover, the LOF allele was not predictive of MACE at 1 year in ACS patients, which has been the centre of much debate in the literature $(228,455-457)$. We hypothesised that the variability in platelet reactivity due to the acute inflammation and oxidative stress following an ACS may be overshadowing the impact of the LOF allele on residual platelet reactivity and measuring the phenotype at a different time point would result in a stronger relationship. However, as genotype is a constant entity and the LOF genotype was not found to be a significant predictor of MACE at 1 year, even if the relationship between HOTPR and CYP2C19*2 was significant at another time point, knowing the genotype of an individual would not aid in risk stratifying a patient. The use of new generation P2Y12 inhibitors may also make the role of bedside genotyping in ACS patients redundant. Ticagrelor is an active drug and does not require hepatic bioactiviation (213). Although the metabolism of prasugrel is via various CYP isoenzymes, the common genetic variants in the CYP2C19 gene do not affect formation of the active metabolite, inhibition of platelet aggregation, or the clinical outcome of prasugrel-treated patients (458). Therefore, genotyping may offer little prognostic information to help clinicians tailor clopidogrel or new antiplatelet therapies to high risk individuals.

In contrast to the ACS population, our study investigating the use of biomarkers to predict risk in patients undergoing major vascular surgery did not find a significant relationship between residual platelet reactivity and outcome. As discussed, this may be due to the stable diseased population that we measured the biomarker in. In CAD patients, platelet function testing is more predictive in patients at a higher risk of stent thrombosis and other thrombotic complications (455) which mainly includes the ACS population undergoing $\mathrm{PCl}$. Our study suggests that platelet function testing does not provide any additional information regarding risk in a stable vascular population. Whether, platelet function testing may be of value in a higher risk acute vascular 
population is unknown. Patients with critical limb ischaemia undergoing femoral bypass surgery and endovascular procedures are at high risks of graft occlusion and limb amputation (122). The risk of limb amputation and death, also increases significantly with every revascularisation procedure (123). Furthermore, studies have demonstrated patients with active limb ischaemia have the highest levels of platelet and monocyte activation before and during surgery compared to those with intermittent claudication (460). High residual platelet reactivity in this population may be more predictive of thrombotic complications and could be used to identify patients who would benefit from more aggressive antiplatelet therapy and prevent further graft complications.

A biomarker that was found to be a significant predictor of ischaemia in the vascular population was an elevated baseline hs-TnT. This is the first study to demonstrate that preoperative measurements of hs-TnT can predict risk in vascular patients. Many studies have found both troponin elevations perioperatively and peak troponin levels post-surgery significantly correlate with an increased risk of mortality following major noncardiac surgery $(461,462)$. However, the clinical utility of measuring this biomarker during surgery is limited to only informing post-operative management. Having a pre-operative biomarker that predicts both short and long-term risk allows clinicians to alter several aspects of patient management to reduce risk. Before surgery, having this information may allow clinicians to carry out further cardiac investigations to optimise medical therapy or change a clinician's strategy regarding surgery and the risk-benefit balance. This information is also valuable for perioperative management as surgical teams can control blood pressure within a stricter range, have blood products available and more aggressive fluid management in patients who are at an increased risk of ischaemia. Whether this approach results in the reduction of ischaemia is unknown and warrants further investigation.

We also demonstrated that platelets have a significant influence on CD4 T cells and this stimulation can be eliminated with prasugrel administration. This stimulatory communication between platelets and T cells may be one of the mechanisms driving risk in patients with HOTPR, resulting in more inflammation and plaque instability. To 
investigate this hypothesis we would first need to look at platelet-CD4 T cell interactions in patients with atherosclerotic disease to see if platelets are having the same effect on the CD4 subsets. It would also be interesting to identify how the activation phenotype of isolated CD4 T cells from patients with atherosclerotic disease effected cytokine production and proliferation compared to healthy volunteers. As we demonstrated in chapter 7, the activation phenotype of CD4 T cells switched from a predominantly memory phenotype in healthy individuals to an effector response in patients with vascular disease. Our finding that platelets enhanced CD4 T cell differentiation and cytokine production but had little impact on proliferation, may suggest platelets are more prone to enhance CD4 T effector-cell responses. Effector cells are primed to make cytokines (463) and platelet interactions in vascular patients may result in even higher levels of cytokine production and CD4 T cell differentiation contributing to the pro-inflammatory environment. The administration of prasugrel in these patients could provide powerful anti-inflammatory actions, which could benefit a patient's long-term outcome and is an important focus of future studies.

We focused our investigation on the interactions between CD4 T cells and platelets. However, it would also be important to understand what effects platelets were having on the activation and function of other T cells including CD8 and NK T cells and B cells in both healthy volunteers and patients with atherosclerosis.

The clinical implications of our study on platelet-CD4 T cell interactions not only relate to atherosclerosis and high on treatment platelet reactivity but may also have important implications in other disease states. Platelets are thought to promote neutrophil and lymphocyte recruitment and cause tissue damage in diseases such as asthma, cystic fibrosis, inflammatory bowel disease, glomerulonephritis and arthritis, from studies conducted in animal and human in vitro models (449). But little is known about how platelets influence lymphocyte function in these diseases from human models and if antiplatelet therapy could provide immunomodulatory benefits. Studies looking at the role of platelets in cancer have primarily focused at their ability to cause thrombotic complications and the release of growth factors supporting tumour growth, angiogenesis and metastasis (464). The dysfunction of the immune system 
and the imbalance between cytotoxic T-cell and regulatory $T$ cell responses can allow cancer tumours to grow unchecked. Platelets may have important effects on T cell responses in cancer and warrant investigation.

Platelets play a key role in sepsis pathophysiology and are one of the drivers of multiple organ failure during systemic infection and inflammation (461). There is substantial evidence that platelets create a pro-coagulant environment in sepsis and aid in the recruitment of leukocytes. However, their direct influence on the activation and phenotype of leukocytes, including T cells have not been investigated (462). There is considerable interest around the use of antiplatelet therapy in sepsis to reduce multiple organ failure. Retrospective studies have investigated the impact of antiplatelet agents on outcomes following critical systemic infections in populations receiving antiplatelet therapy for other indications. These studies have found a trend to shorter ICU stays following infection, reduced rates of acute lung injury and in some cases, reduced mortality in patients receiving antiplatelet therapy (462). Antiplatelet therapy is undoubtedly lowering the thrombotic ability of platelets and reducing their propensity to cause pro-coagulant environments in sepsis. Another potential benefit of antiplatelet therapy in sepsis, which merits further investigation, may come from inhibiting platelet's communication with immune cells and reducing the proinflammatory environment.

Platelets may have important roles in a number of other diseases not mentioned here. Their high frequency, ability to bind and communicate with an array of cells and abundant stores of cytokines, chemokines and growth factors mean platelets are positioned to significantly influence the immune system in a wide range of diseases. 
${ }_{10}$ References 
1. Stary HC, Chandler AB, Dinsmore RE, Fuster V, Glagov S, Insull W, et al. A Definition of Advanced Types of Atherosclerotic Lesions and a Histological Classification of Atherosclerosis: A Report From the Committee on Vascular Lesions of the Council on Arteriosclerosis, American Heart Association. Arteriosclerosis, Thrombosis, and Vascular Biology. 1995;15(9):1512-31.

2. Stary HC, Chandler AB, Glagov S, Guyton JR, Insull W, Rosenfeld ME, et al. A definition of initial, fatty streak, and intermediate lesions of atherosclerosis. A report from the Committee on Vascular Lesions of the Council on Arteriosclerosis, American Heart Association. Circulation. 1994;89(5):246278.

3. Nakashima Y, Raines EW, Plump AS, Breslow JL, Ross R. Upregulation of VCAM-1 and ICAM-1 at Atherosclerosis-Prone Sites on the Endothelium in the ApoE-Deficient Mouse. Arteriosclerosis, Thrombosis, and Vascular Biology. 1998;18(5):842-51.

4. Vion A-Cm, Ramkhelawon B, Loyer X, Chironi G, Devue C, Loirand G, et al. Shear Stress Regulates Endothelial Microparticle Release. Circulation Research. 2013;112(10):1323-33.

5. Oxidized phospholipids as modulators of inflammation in atherosclerosis. Curr Opin Lipidol. 2003;14(5):421-30.

6. Massberg S, Brand $\mathrm{K}, \mathrm{Gr} \sqrt{ }^{\mathrm{o}}$ ner S, Page S, $\mathrm{M} \sqrt{ }^{\mathrm{O}}$ Iler $\mathrm{E}, \mathrm{M} \sqrt{ }^{\mathrm{O}}$ ller I, et al. A Critical Role of Platelet Adhesion in the Initiation of Atherosclerotic Lesion Formation. The Journal of Experimental Medicine. 2002;196(7):887-96.

7. Hansson GK. Inflammation, atherosclerosis, and coronary artery disease. N Engl J Med. 2005;352(16):1685-95. Epub 2005/04/22.

8. Jonasson L, Holm J, Skalli O, Bondjers G, Hansson GK. Regional accumulations of T cells, macrophages, and smooth muscle cells in the human atherosclerotic plaque. Arteriosclerosis, Thrombosis, and Vascular Biology. 1986;6(2):131-8.

9. Kovanen PT, Kaartinen M, Paavonen T. Infiltrates of Activated Mast Cells at the Site of Coronary Atheromatous Erosion or Rupture in Myocardial Infarction. Circulation. 1995;92(5):1084-8.

10. Glagov S, Weisenberg E, Zarins CK, Stankunavicius R, Kolettis GJ. Compensatory enlargement of human atherosclerotic coronary arteries. N Engl J Med. 1987;316(22):1371-5. Epub 1987/05/28.

11. Smith JD, Trogan E, Ginsberg M, Grigaux C, Tian J, Miyata M. Decreased atherosclerosis in mice deficient in both macrophage colony-stimulating factor (op) and apolipoprotein E. Proceedings of the National Academy of Sciences. 1995;92(18):8264-8.

12. Peiser L, Mukhopadhyay S, Gordon S. Scavenger receptors in innate immunity. Current Opinion in Immunology. 2002;14(1):123-8.

13. Janeway CA, Jr., Medzhitov R. Innate immune recognition. Annu Rev Immunol. 2002;20:197216. Epub 2002/02/28.

14. Xu Q. Role of heat shock proteins in atherosclerosis. Arteriosclerosis, Thrombosis, and Vascular Biology. 2002;22(10):1547-59. Epub 2002/10/16.

15. Stemme S, Faber B, Holm J, Wiklund O, Witztum JL, Hansson GK. T Iymphocytes from human atherosclerotic plaques recognize oxidized low density lipoprotein. Proc Natl Acad Sci U S A. 1995;92(9):3893-7. Epub 1995/04/25.

16. Hansson GK. Immune mechanisms in atherosclerosis. Arteriosclerosis, Thrombosis, and Vascular Biology. 2001;21(12):1876-90. Epub 2001/12/18.

17. Uyemura K, Demer LL, Castle SC, Jullien D, Berliner JA, Gately MK, et al. Cross-regulatory roles of interleukin (IL)-12 and IL-10 in atherosclerosis. J Clin Invest. 1996;97(9):2130-8. Epub 1996/05/01.

18. Frostegard J, Ulfgren A-K, Nyberg P, Hedin U, Swedenborg J, Andersson U, et al. Cytokine expression in advanced human atherosclerotic plaques: dominance of pro-inflammatory (Th1) and macrophage-stimulating cytokines. Atherosclerosis. 1999;145(1):33-43.

19. Szabo SJ, Sullivan BM, Peng SL, Glimcher LH. Molecular mechanisms regulating Th1 immune responses. Annu Rev Immunol. 2003;21:713-58. Epub 2002/12/26.

20. Cheng X, Yu X, Ding YJ, Fu QQ, Xie JJ, Tang TT, et al. The Th17/Treg imbalance in patients with acute coronary syndrome. Clin Immunol. 2008;127(1):89-97.

21. Gao Q, Jiang Y, Ma T, Zhu F, Gao F, Zhang P, et al. A critical function of Th17 proinflammatory cells in the development of atherosclerotic plaque in mice. J Immunol. 2010;185(10):5820-7. Epub 2010/10/19.

22. Taleb S, Romain M, Ramkhelawon B, Uyttenhove C, Pasterkamp G, Herbin O, et al. Loss of SOCS3 Expression in T Cells Reveals a Regulatory Role for Interleukin-17 in Atherosclerosis. Circulation. 2009;120(18):S1064-S5. 
23. de Boer OJ, van der Meer JJ, Teeling P, van der Loos CM, van der Wal AC. Low Numbers of FOXP3 Positive Regulatory T Cells Are Present in all Developmental Stages of Human Atherosclerotic Lesions. PLoS ONE. 2007;2(8).

24. Mor A, Luboshits G, Planer D, Keren G, George J. Altered status of CD4(+)CD25(+) regulatory T cells in patients with acute coronary syndromes. European Heart Journal. 2006;27(21):2530-7. Epub 2006/09/07.

25. Ait-Oufella H, Salomon BL, Potteaux S, Robertson AK, Gourdy P, Zoll J, et al. Natural regulatory $T$ cells control the development of atherosclerosis in mice. Nat Med. 2006;12(2):178-80. Epub 2006/02/08.

26. Heller EA, Liu E, Tager AM, Yuan Q, Lin AY, Ahluwalia N, et al. Chemokine CXCL10 promotes atherogenesis by modulating the local balance of effector and regulatory $\mathrm{T}$ cells. Circulation. 2006;113(19):2301-12. Epub 2006/05/10.

27. Sasaki N, Yamashita T, Takeda M, Shinohara M, Nakajima K, Tawa H, et al. Oral anti-CD3 antibody treatment induces regulatory $\mathrm{T}$ cells and inhibits the development of atherosclerosis in mice. Circulation. 2009;120(20):1996-2005. Epub 2009/11/04.

28. Steffens S, Burger F, Pelli G, Dean Y, Elson G, Kosco-Vilbois M, et al. Short-term treatment with anti-CD3 antibody reduces the development and progression of atherosclerosis in mice. Circulation. 2006;114(18):1977-84. Epub 2006/10/18.

29. Tupin E, Nicoletti A, Elhage R, Rudling M, Ljunggren HG, Hansson GK, et al. CD1d-dependent activation of NKT cells aggravates atherosclerosis. The Journal of Experimental Medicine. 2004;199(3):417-22. Epub 2004/01/28.

30. Ludewig B, Freigang $S, J \sqrt{ } \S$ ggi M, Kurrer MO, Pei Y-C, Vlk L, et al. Linking immune-mediated arterial inflammation and cholesterol-induced atherosclerosis in a transgenic mouse model. Proceedings of the National Academy of Sciences. 2000;97(23):12752-7.

31. Hackett D, Davies G, Maseri A. Pre-existing coronary stenoses in patients with first myocardial infarction are not necessarily severe. European Heart Journal. 1988;9(12):1317-23. Epub 1988/12/01.

32. Motoyama S, Sarai M, Harigaya H, Anno H, Inoue K, Hara T, et al. Computed tomographic angiography characteristics of atherosclerotic plaques subsequently resulting in acute coronary syndrome. J Am Coll Cardiol. 2009;54(1):49-57. Epub 2009/06/27.

33. Hoffmann U, Moselewski F, Nieman K, Jang IK, Ferencik M, Rahman AM, et al. Noninvasive assessment of plaque morphology and composition in culprit and stable lesions in acute coronary syndrome and stable lesions in stable angina by multidetector computed tomography. J Am Coll Cardiol. 2006;47(8):1655-62. Epub 2006/04/25.

34. Arbab-Zadeh A, Nakano M, Virmani R, Fuster V. Acute coronary events. Circulation. 2012;125(9):1147-56. Epub 2012/03/07.

35. Davies MJ. Stability and Instability: Two Faces of Coronary Atherosclerosis: The Paul Dudley White Lecture 1995. Circulation. 1996;94(8):2013-20.

36. Falk E, Shah PK, Fuster V. Coronary plaque disruption. Circulation. 1995;92(3):657-71. Epub 1995/08/01.

37. Yonetsu T, Kakuta T, Lee T, Takahashi K, Kawaguchi N, Yamamoto G, et al. In vivo critical fibrous cap thickness for rupture-prone coronary plaques assessed by optical coherence tomography. European Heart Journal. 2011;32(10):1251-9. Epub 2011/01/29.

38. Amento EP, Ehsani N, Palmer H, Libby P. Cytokines and growth factors positively and negatively regulate interstitial collagen gene expression in human vascular smooth muscle cells. Arterioscler Thromb. 1991;11(5):1223-30. Epub 1991/09/01.

39. Nikkari ST, O'Brien KD, Ferguson M, Hatsukami T, Welgus HG, Alpers CE, et al. Interstitial collagenase (MMP-1) expression in human carotid atherosclerosis. Circulation. 1995;92(6):1393-8. Epub 1995/09/15.

40. Libby P. Mechanisms of acute coronary syndromes and their implications for therapy. N Engl J Med. 2013;368(21):2004-13. Epub 2013/05/24.

41. Libby P. The molecular mechanisms of the thrombotic complications of atherosclerosis. J Intern Med. 2008;263(5):517-27. Epub 2008/04/16.

42. Sugiyama S, Kugiyama K, Aikawa M, Nakamura S, Ogawa H, Libby P. Hypochlorous acid, a macrophage product, induces endothelial apoptosis and tissue factor expression: involvement of myeloperoxidase-mediated oxidant in plaque erosion and thrombogenesis. Arteriosclerosis, Thrombosis, and Vascular Biology. 2004;24(7):1309-14. Epub 2004/05/15. 
43. Falk E, Nakano M, Bentzon JF, Finn AV, Virmani R. Update on acute coronary syndromes: the pathologists' view. European Heart Journal. 2013;34(10):719-28. Epub 2012/12/18.

44. Finn AV, Nakano M, Narula J, Kolodgie FD, Virmani R. Concept of vulnerable/unstable plaque. Arteriosclerosis, Thrombosis, and Vascular Biology. 2010;30(7):1282-92. Epub 2010/06/18.

45. Davies MJ. The contribution of thrombosis to the clinical expression of coronary atherosclerosis. Thrombosis Research. 1996;82(1):1-32.

46. Karras C, Donlan SM, Aitchison R, Aitchison P, Wang E, Kharasch M. Acute coronary syndromes. Disease-a-Month. 2013;59(5):202-9.

47. Hirsch AT, Criqui MH, Treat-Jacobson D, Regensteiner JG, Creager MA, Olin JW, et al. Peripheral arterial disease detection, awareness, and treatment in primary care. Jama. 2001;286(11):1317-24. Epub 2001/09/19.

48. Hirsch AT, Haskal ZJ, Hertzer NR, Bakal CW, Creager MA, Halperin JL, et al. ACC/AHA 2005 Practice Guidelines for the management of patients with peripheral arterial disease (lower extremity, renal, mesenteric, and abdominal aortic): a collaborative report from the American Association for Vascular Surgery/Society for Vascular Surgery, Society for Cardiovascular Angiography and Interventions, Society for Vascular Medicine and Biology, Society of Interventional Radiology, and the ACC/AHA Task Force on Practice Guidelines (Writing Committee to Develop Guidelines for the Management of Patients With Peripheral Arterial Disease): endorsed by the American Association of Cardiovascular and Pulmonary Rehabilitation; National Heart, Lung, and Blood Institute; Society for Vascular Nursing; TransAtlantic Inter-Society Consensus; and Vascular Disease Foundation. Circulation. 2006;113(11):e463-654. Epub 2006/03/22.

49. Steg PG, Bhatt DL, Wilson PW, D'Agostino R, Sr., Ohman EM, Rother J, et al. One-year cardiovascular event rates in outpatients with atherothrombosis. Jama. 2007;297(11):1197-206. Epub 2007/03/22.

50. Golomb BA, Dang TT, Criqui MH. Peripheral arterial disease: morbidity and mortality implications. Circulation. 2006;114(7):688-99. Epub 2006/08/16.

51. Cao JJ, Arnold AM, Manolio TA, Polak JF, Psaty BM, Hirsch CH, et al. Association of carotid artery intima-media thickness, plaques, and C-reactive protein with future cardiovascular disease and all-cause mortality: the Cardiovascular Health Study. Circulation. 2007;116(1):32-8. Epub 2007/06/20.

52. Barnett HJ, Gunton RW, Eliasziw M, Fleming L, Sharpe B, Gates P, et al. Causes and severity of ischemic stroke in patients with internal carotid artery stenosis. Jama. 2000;283(11):1429-36. Epub 2000/03/25.

53. Easton JD, Saver JL, Albers GW, Alberts MJ, Chaturvedi S, Feldmann E, et al. Definition and evaluation of transient ischemic attack: a scientific statement for healthcare professionals from the American Heart Association/American Stroke Association Stroke Council; Council on Cardiovascular Surgery and Anesthesia; Council on Cardiovascular Radiology and Intervention; Council on Cardiovascular Nursing; and the Interdisciplinary Council on Peripheral Vascular Disease. The American Academy of Neurology affirms the value of this statement as an educational tool for neurologists. Stroke. 2009;40(6):2276-93. Epub 2009/05/09.

54. Halliday A, Mansfield A, Marro J, Peto C, Peto R, Potter J, et al. Prevention of disabling and fatal strokes by successful carotid endarterectomy in patients without recent neurological symptoms: randomised controlled trial. Lancet. 2004;363(9420):1491-502. Epub 2004/05/12.

55. Sila CA, Higashida RT, Clagett GP. Clinical decisions. Management of carotid stenosis. N Engl J Med. 2008;358(15):1617-21. Epub 2008/04/12.

56. Edwards AT, Blann AD, Suarez-Mendez VJ, Lardi AM, McCollum CN. Systemic responses in patients with intermittent claudication after treadmill exercise. Br J Surg. 1994;81(12):1738-41. Epub 1994/12/01.

57. Belch JJF, McLaren M, Khan F, Hickman P, Muir A, Stonebridge $P$. The inflammatory process in intermittent claudication. Eur Heart J Suppl. 2002;4(B):B31-B4.

58. Neumann FJ, Waas W, Diehm C, Weiss T, Haupt HM, Zimmermann R, et al. Activation and Decreased Deformability of Neutrophils after Intermittent Claudication. Circulation. 1990;82(3):922-9. 59. Hirsch AT, Haskal ZJ, Hertzer NR, Bakal CW, Creager MA, Halperin JL, et al. ACC/AHA 2005 guidelines for the management of patients with peripheral arterial disease (lower extremity, renal, mesenteric, and abdominal aortic): executive summary a collaborative report from the American Association for Vascular Surgery/Society for Vascular Surgery, Society for Cardiovascular Angiography and Interventions, Society for Vascular Medicine and Biology, Society of Interventional Radiology, and the ACC/AHA Task Force on Practice Guidelines (Writing Committee to Develop Guidelines for the 
Management of Patients With Peripheral Arterial Disease) endorsed by the American Association of Cardiovascular and Pulmonary Rehabilitation; National Heart, Lung, and Blood Institute; Society for Vascular Nursing; TransAtlantic Inter-Society Consensus; and Vascular Disease Foundation. J Am Coll Cardiol. 2006;47(6):1239-312. Epub 2006/03/21.

60. Teodorescu VJ, Vavra AK, Kibbe MR. Peripheral arterial disease in women. J Vasc Surg. 2013;57(4 Suppl):18S-26S. Epub 2013/04/03.

61. Neumann FJ, Waas W, Diehm C, Weiss T, Haupt HM, Zimmermann R, et al. Activation and decreased deformability of neutrophils after intermittent claudication. Circulation. 1990;82(3):922-9. Epub 1990/09/01.

62. Giri J, McDermott MM, Greenland P, Guralnik JM, Criqui MH, Liu K, et al. Statin use and functional decline in patients with and without peripheral arterial disease. J Am Coll Cardiol. 2006;47(5):998-1004.

63. Gent M, Beaumont D, Blanchard J, Bousser MG, Coffman J, Easton JD, et al. A randomised, blinded, trial of clopidogrel versus aspirin in patients at risk of ischaemic events (CAPRIE). Lancet. 1996;348(9038):1329-39.

64. Berger JS, Krantz MJ, Kittelson JM, Hiatt WR. Aspirin for the Prevention of Cardiovascular Events in Patients With Peripheral Artery Disease A Meta-analysis of Randomized Trials. Jama-J Am Med Assoc. 2009;301(18):1909-19.

65. Kniemeyer HW, Kessler T, Reber PU, Ris HB, Hakki H, Widmer MK. Treatment of ruptured abdominal aortic aneurysm, a permanent challenge or a waste of resources? Prediction of outcome using a multi-organ-dysfunction score. Eur J Vasc Endovasc. 2000;19(2):190-6.

66. Xu CP, Zarins CK, Glagov S. Aneurysmal and occlusive atherosclerosis of the human abdominal aorta. J Vasc Surg. 2001;33(1):91-6.

67. Carrell TWG, Burnand KG, Wells GMA, Clements JM, Smith A. Stromelysin-1 (matrix metalloproteinase-3) and tissue inhibitor of metalloproteinase-3 are overexpressed in the wall of abdominal aortic aneurysms. Circulation. 2002;105(4):477-82.

68. Defawe OD, Colige A, Lambert CA, Munaut C, Delvenne P, Lapiere CM, et al. TIMP-2 and PAI-1 mRNA levels are lower in aneurysmal as compared to athero-occlusive abdominal aortas. Cardiovascular Research. 2003;60(1):205-13.

69. Sakalihasan N, Limet R, Defawe OD. Abdominal aortic aneurysm. Lancet. 2005;365(9470):1577-89. Epub 2005/05/04.

70. Vorp DA, Lee PC, Wang DH, Makaroun MS, Nemoto EM, Ogawa S, et al. Association of intraluminal thrombus in abdominal aortic aneurysm with local hypoxia and wall weakening. J Vasc Surg. 2001;34(2):291-9. Epub 2001/08/10.

71. Sakalihasan N, Delvenne P, Nusgens BV, Limet R, Lapiere CM. Activated forms of MMP2 and MMP9 in abdominal aortic aneurysms. J Vasc Surg. 1996;24(1):127-33. Epub 1996/07/01.

72. Vardulaki KA, Walker NM, Day NE, Duffy SW, Ashton HA, Scott RAP. Quantifying the risks of hypertension, age, sex and smoking in patients with abdominal aortic aneurysm. Brit J Surg. 2000;87(2):195-200.

73. Blanchard JF, Armenian HK, Friesen PP. Risk factors for abdominal aortic aneurysm: Results of a case-control study. Am J Epidemiol. 2000;151(6):575-83.

74. Lederle FA, Nelson DB, Joseph AM. Smokers' relative risk for aortic aneurysm compared with other smoking-related diseases: a systematic review. J Vasc Surg. 2003;38(2):329-34. Epub 2003/08/02. 75. Semple JW, Italiano JE, Freedman J. Platelets and the immune continuum. Nature Reviews Immunology. 2011;11(4):264-74.

76. Hartwig JH. Mechanisms of Actin Rearrangements Mediating Platelet Activation. J Cell Biol. 1992;118(6):1421-42.

77. Bennett JS. Structure and function of the platelet integrin alpha(Ilb)beta(3). Journal of Clinical Investigation. 2005;115(12):3363-9.

78. Lievens D, von Hundelshausen P. Platelets in atherosclerosis. Thromb Haemost. 2011;106(5):827-38.

79. Massberg S, Brand K, Gruner S, Page S, Muller E, Muller I, et al. A critical role of platelet adhesion in the initiation of atherosclerotic lesion formation. The Journal of Experimental Medicine. 2002;196(7):887-96. Epub 2002/10/09.

80. Denis CV, Wagner DD. Platelet adhesion receptors and their ligands in mouse models of thrombosis. Arterioscl Throm Vas. 2007;27(4):728-39. 
81. Bigalke B, Lindemann S, Ehlers R, Seizer P, Daub K, Langer H, et al. Expression of platelet collagen receptor glycoprotein $\mathrm{VI}$ is associated with acute coronary syndrome. European Heart Journal. 2006;27(18):2165-9.

82. Wang YM, Sakuma M, Chen ZP, Ustinov V, Shi C, Croce K, et al. Leukocyte engagement of platelet glycoprotein Ib alpha via the integrin Mac-1 is critical for the biological response to vascular injury. Circulation. 2005;112(19):2993-3000.

83. Bultmann A, Li ZM, Wagner S, Peluso M, Schonberger T, Weis C, et al. Impact of glycoprotein $\mathrm{VI}$ and platelet adhesion on atherosclerosis-A possible role of fibronectin. J Mol Cell Cardiol. 2010;49(3):532-42.

84. Schulz C, von Bruhl ML, Barocke V, Cullen P, Mayer K, Okrojek R, et al. EMMPRIN (CD147/basigin) mediates platelet-monocyte interactions in vivo and augments monocyte recruitment to the vascular wall. Journal of Thrombosis and Haemostasis. 2011;9(5):1007-19.

85. Massberg S, Schurzinger K, Lorenz M, Konrad I, Schulz C, Plesnila N, et al. Platelet adhesion via glycoprotein Ilb integrin is critical for atheroprogression and focal cerebral ischemia - An in vivo study in mice lacking glycoprotein IIb. Circulation. 2005;112(8):1180-8.

86. Bigalke B, Stellos K, Geisler T, Kremmer E, Seizer P, May AE, et al. Glycoprotein VI for diagnosis of acute coronary syndrome when ECG is ambiguous. Int J Cardiol. 2011;149(2):164-8.

87. Dong ZM, Brown AA, Wagner DD. Prominent role of P-selectin in the development of advanced atherosclerosis in apoE-deficient mice. Circulation. 2000;101(19):2290-5.

88. Dole VS, Bergmeier W, Mitchell HA, Eichenberger SC, Wagner DD. Activated platelets induce Weibel-Palade-body secretion and leukocyte rolling in vivo: role of P-selectin. Blood. 2005;106(7):23349.

89. Huo YQ, Schober A, Forlow SB, Smith DF, Hyman MC, Jung S, et al. Circulating activated platelets exacerbate atherosclerosis in mice deficient in apolipoprotein E. Nat Med. 2003;9(1):61-7.

90. van Gils JM, Zwaginga JJ, Hordijk PL. Molecular and functional interactions among monocytes, platelets, and endothelial cells and their relevance for cardiovascular diseases. J Leukoc Biol. 2009;85(2):195-204.

91. Ridker PM, Buring JE, Rifai N. Soluble P-selectin and the risk of future cardiovascular events. Circulation. 2001;103(4):491-5.

92. Kisucka J, Chauhan AK, Zhao BQ, Patten IS, Yesilaltay A, Krieger M, et al. Elevated levels of soluble P-selectin in mice alter blood-brain barrier function, exacerbate stroke, and promote atherosclerosis. Blood. 2009;113(23):6015-22.

93. Engel D, Seijkens T, Poggi M, Sanati M, Thevissen L, Beckers L, et al. The immunobiology of CD154-CD40-TRAF interactions in atherosclerosis. Semin Immunol. 2009;21(5):308-12.

94. Lievens D, Eijgelaar WJ, Biessen EAL, Daemen MJAP, Lutgens E. The multi-functionality of CD40L and its receptor CD40 in atherosclerosis. Thromb Haemost. 2009;102(2):206-14.

95. Henn V, Slupsky JR, Grafe M, Anagnostopoulos I, Forster R, Muller-Berghaus G, et al. CD40 ligand on activated platelets triggers an inflammatory reaction of endothelial cells. Nature. 1998;391(6667):591-4.

96. Zhou XH, Nicoletti A, Elhage R, Hansson GK. Transfer of CD4(+) T cells aggravates atherosclerosis in immunodeficient apolipoprotein E knockout mice. Circulation. 2000;102(24):291922.

97. Ait-Oufella H, Salomon BL, Potteaux S, Robertson AKL, Gourdy P, Zoll J, et al. Natural regulatory T cells control the development of atherosclerosis in mice. Nat Med. 2006;12(2):178-80.

98. Edfeldt K, Swedenborg J, Hansson GK, Yan ZQ. Expression of toll-like receptors in human atherosclerotic lesions - A possible pathway for plaque activation. Circulation. 2002;105(10):1158-61.

99. Clemetson KJ, Clemetson JM, Proudfoot AEI, Power CA, Baggiolini M, Wells TNC. Functional expression of CCR1, CCR3, CCR4, and CXCR4 chemokine receptors on human platelets. Blood. 2000;96(13):4046-54.

100. Assinger A, Koller F, Schmid W, Zellner M, Koller E, Volf I. Hypochlorite-oxidized LDL induces intraplatelet ROS formation and surface exposure of CD40L-A prominent role of CD36. Atherosclerosis. 2010;213(1):129-34.

101. Schall TJ, Bacon K, Toy KJ, Goeddel DV. Selective Attraction of Monocytes and Lymphocytes-T of the Memory Phenotype by Cytokine Rantes. Nature. 1990;347(6294):669-71.

102. Brandt E, Ludwig A, Petersen F, Flad HD. Platelet-derived CXC chemokines: old players in new games. Immunol Rev. 2000;177:204-16. 
103. Koenen RR, von Hundelshausen P, Nesmelova IV, Zernecke A, Liehn EA, Sarabi A, et al. Disrupting functional interactions between platelet chemokines inhibits atherosclerosis in hyperlipidemic mice. Nat Med. 2009;15(1):97-103.

104. von Hundelshausen P, Weber KSC, Huo YQ, Proudfoot AEI, Nelson PJ, Ley K, et al. RANTES deposition by platelets triggers monocyte arrest on inflamed and atherosclerotic endothelium. Circulation. 2001;103(13):1772-7.

105. Merhi-Soussi F, Kwak BR, Magne D, Chadjichristos C, Berti M, Pelli G, et al. Interleukin-1 plays a major role in vascular inflammation and atherosclerosis in male apolipoprotein E-knockout mice. Cardiovascular Research. 2005;66(3):583-93.

106. Kaplanski G, Farnarier C, Kaplanski S, Porat R, Shapiro L, Bongrand P, et al. Interleukin-1 Induces Interleukin-8 Secretion from Endothelial-Cells by a Juxtacrine Mechanism. Blood. 1994;84(12):4242-8.

107. Gawaz M, Brand K, Dickfeld T, Pogatsa-Murray G, Page S, Bogner C, et al. Platelets induce alterations of chemotactic and adhesive properties of endothelial cells mediated through an interleukin-1-dependent mechanism. Implications for atherogenesis. Atherosclerosis. 2000;148(1):7585.

108. Toma I, McCaffrey TA. Transforming growth factor-beta and atherosclerosis: interwoven atherogenic and atheroprotective aspects. Cell Tissue Res. 2012;347(1):155-75.

109. Lutgens $E$, Gijbels M, Smook M, Heeringa $P$, Gotwals $P$, Koteliansky VE, et al. Transforming growth factor-beta mediates balance between inflammation and fibrosis during plaque progression. Arterioscl Throm Vas. 2002;22(6):975-82.

110. Ruggeri ZM, Jackson SP. Chapter 20 - Platelet Thrombus Formation in Flowing Blood. Platelets (Third Edition): Academic Press; 2013. p. 399-423.

111. Jackson SP. The growing complexity of platelet aggregation. Blood. 2007;109(12):5087-95. Epub 2007/02/22.

112. van Gestel MA, Heemskerk JWM, Slaaf DW, Heijnen VVT, Sage SO, Reneman RS, et al. Realtime detection of activation patterns in individual platelets during thromboembolism in vivo: Differences between thrombus growth and embolus formation. J Vasc Res. 2002;39(6):534-43.

113. Dubois C, Panicot-Dubois L, Gainor JF, Furie BC, Furie B. Thrombin-initiated platelet activation in vivo is vWF independent during thrombus formation in a laser injury model. Journal of Clinical Investigation. 2007;117(4):953-60.

114. Maxwell MJ, Westein E, Nesbitt WS, Giuliano S, Dopheide SM, Jackson SP. Identification of a 2-stage platelet aggregation process mediating shear-dependent thrombus formation. Blood. 2007;109(2):566-76. Epub 2006/09/23.

115. Brass LF, Newman DK, Wannermacher KM, Zhu L, Stalker TJ. Chapter 19 - Signal Transduction During Platelet Plug Formation. Platelets (Third Edition): Academic Press; 2013. p. 367-98.

116. Holmes DR, Kereiakes DJ, Laskey WK, Colombo A, Ellis SG, Henry TD, et al. Thrombosis and drug-eluting stents - An objective appraisal. J Am Coll Cardiol. 2007;50(2):109-18.

117. Kirtane AJ, Gupta A, lyengar S, Moses JW, Leon MB, Applegate R, et al. Safety and Efficacy of Drug-Eluting and Bare Metal Stents Comprehensive Meta-Analysis of Randomized Trials and Observational Studies. Circulation. 2009;119(25):3198-U78.

118. de la Torre-Hernandez JM, Alfonso F, Hernandez F, Elizaga J, Sanmartin M, Pinar E, et al. Drugeluting stent thrombosis: results from the multicenter Spanish registry ESTROFA (Estudio ESpanol sobre TROmbosis de stents FArmacoactivos). J Am Coll Cardiol. 2008;51(10):986-90. Epub 2008/03/08.

119. Leibundgut G, Nietlispach F, PittI U, Rocca HBL, Kaiser CA, Pfisterer ME. Stent thrombosis up to 3 years after stenting for ST-segment elevation myocardial infarction versus for stable anginaComparison of the effects of drug-eluting versus bare-metal stents. Am Heart J. 2009;158(2):271-6.

120. Holmes DR, Kereiakes DJ, Garg S, Serruys PW, Dehmer GJ, Ellis SG, et al. Stent Thrombosis. J Am Coll Cardiol. 2010;56(17):1357-65.

121. Oresanya L, Makam AN, Belkin M, Moneta GL, Conte MS. Factors associated with primary vein graft occlusion in a multicenter trial with mandated ultrasound surveillance. J Vasc Surg. 2013. Epub 2013/12/24.

122. Byrne RM, Taha AG, Avgerinos E, Marone LK, Makaroun MS, Chaer RA. Contemporary outcomes of endovascular interventions for acute limb ischemia. J Vasc Surg. 2013. Epub 2013/12/24.

123. Hamm CW, Bassand JP, Agewall S, Bax J, Boersma E, Bueno H, et al. ESC Guidelines for the management of acute coronary syndromes in patients presenting without persistent ST-segment elevation. European Heart Journal. 2011;32(23):2999-3054. 
124. Fox Ka SPEKA, et al. DEcline in rates of death and heart failure in acute coronary syndromes, 1999-2006. Jama. 2007;297(17):1892-900.

125. Kolh P, Wijns W, Danchin N, Di Mario C, Falk V, Folliguet T, et al. Guidelines on myocardial revascularization The Task Force on Myocardial Revascularization of the European Society of Cardiology (ESC) and the European Association for Cardio-Thoracic Surgery (EACTS). Eur J Cardio-Thorac. 2010;38:S1-S52.

126. Jneid H, Anderson JL, Wright RS, Adams CD, Bridges CR, Casey DE, et al. 2012 ACCF/AHA Focused Update of the Guideline for the Management of Patients With Unstable Angina/Non-STElevation Myocardial Infarction (Updating the 2007 Guideline and Replacing the 2011 Focused Update) A Report of the American College of Cardiology Foundation/American Heart Association Task Force on Practice Guidelines Developed in Collaboration With the American College of Emergency Physicians, Society for Cardiovascular Angiography and Interventions, and Society of Thoracic Surgeons. Circulation. 2012;126(7):875-+.

127. Kushner FG. 2009 Focused Updates: ACC/AHA Guidelines for the Management of Patients With ST-Elevation Myocardial Infarction (Updating the 2004 Guideline and 2007 Focused Update) and ACC/AHA/SCAI Guidelines on Percutaneous Coronary Intervention (Updating the 2005 Guideline and 2007 Focused Update): A Report of the American College of Cardiology Foundation/American Heart Association Task Force on Practice Guidelines (vol 54, pg 2205, 2009). Catheter Cardio Inte. 2010;75(7):1143-.

128. Bhatt DL, Flather MD, Hacke W, Berger PB, Black HR, Boden WE, et al. Patients with prior myocardial infarction, stroke, or symptomatic peripheral arterial disease in the CHARISMA trial. J Am Coll Cardiol. 2007;49(19):1982-8. Epub 2007/05/15.

129. Cacoub PP, Bhatt DL, Steg PG, Topol EJ, Creager MA. Patients with peripheral arterial disease in the CHARISMA trial. Eur Heart J. 2009;30(2):192-201. doi: 10.1093/eurheartj/ehn534. Epub 2009 Jan 9.

130. Patrono C, Garcia Rodriguez LA, Landolfi R, Baigent C. Low-dose aspirin for the prevention of atherothrombosis. N Engl J Med. 2005;353(22):2373-83. Epub 2005/12/02.

131. Diminno G, Silver MJ, Murphy S. Monitoring the Entry of New Platelets into the Circulation after Ingestion of Aspirin. Blood. 1983;61(6):1081-5.

132. Reilly IA, FitzGerald GA. Inhibition of thromboxane formation in vivo and ex vivo: implications for therapy with platelet inhibitory drugs. Blood. 1987;69(1):180-6. Epub 1987/01/01.

133. Rocca B, Secchiero P, Ciabattoni G, Ranelletti FO, Catani L, Guidotti L, et al. Cyclooxygenase-2 expression is induced during human megakaryopoiesis and characterizes newly formed platelets. Proc Natl Acad Sci U S A. 2002;99(11):7634-9.

134. Baigent C, Blackwell L, Collins R, Emberson J, Godwin J, Peto R, et al. Aspirin in the primary and secondary prevention of vascular disease: collaborative meta-analysis of individual participant data from randomised trials. Lancet. 2009;373(9678):1849-60. Epub 2009/06/02.

135. De Berardis G, Sacco M, Strippoli GF, Pellegrini F, Graziano G, Tognoni G, et al. Aspirin for primary prevention of cardiovascular events in people with diabetes: meta-analysis of randomised controlled trials. Bmj. 2009;339:b4531. Epub 2009/11/10.

136. Patrono C, Coller B, Fitzgerald GA, Hirsh J, Roth G. Platelet-active drugs: The relationships among dose, effectiveness, and side effects. Chest. 2004;126(3):234S-64S.

137. Collaborative meta-analysis of randomised trials of antiplatelet therapy for prevention of death, myocardial infarction, and stroke in high risk patients (vol 324, pg 71, 2002). Brit Med J. 2002;324(7330):141-.

138. Risk of myocardial infarction and death during treatment with low dose aspirin and intravenous heparin in men with unstable coronary artery disease. The RISC Group. Lancet. 1990;336(8719):827-30. Epub 1990/10/06.

139. Lewis HD, Jr., Davis JW, Archibald DG, Steinke WE, Smitherman TC, Doherty JE, 3rd, et al. Protective effects of aspirin against acute myocardial infarction and death in men with unstable angina. Results of a Veterans Administration Cooperative Study. N Engl J Med. 1983;309(7):396-403. Epub 1983/08/18.

140. Roux S, Christeller S, Ludin E. Effects of aspirin on coronary reocclusion and recurrent ischemia after thrombolysis: a meta-analysis. J Am Coll Cardiol. 1992;19(3):671-7. Epub 1992/03/01.

141. Snoep JD, Hovens MM, Eikenboom JC, van der Bom JG, Huisman MV. Association of laboratory-defined aspirin resistance with a higher risk of recurrent cardiovascular events: a systematic review and meta-analysis. Arch Intern Med. 2007;167(15):1593-9. Epub 2007/08/19. 
142. Krasopoulos G, Brister SJ, Beattie WS, Buchanan MR. Aspirin "resistance" and risk of cardiovascular morbidity: systematic review and meta-analysis. Bmj. 2008;336(7637):195-8. Epub 2008/01/19.

143. Snoep JD, Hovens MMC, Eikenboom JCJ, van der Bom JG, Huisman MV. Association of laboratory-defined aspirin resistance with a higher risk of recurrent cardiovascular events - A systematic review and meta-analysis. Arch Intern Med. 2007;167(15):1593-9.

144. Biondi-Zoccai GGL, Lotrionte M, Agostoni P, Abbate A, Fusaro M, Burzotta F, et al. A systematic review and meta-analysis on the hazards of discontinuing or not adhering to aspirin among 50279 patients at risk for coronary artery disease. European Heart Journal. 2006;27(22):2667-74.

145. Collaborative meta-analysis of randomised trials of antiplatelet therapy for prevention of death, myocardial infarction, and stroke in high risk patients. Bmj. 2002;324(7329):71-86.

146. Rooke TW, Hirsch AT, Misra S, Sidawy AN, Beckman JA, Findeiss LK, et al. 2011 ACCF/AHA Focused Update of the Guideline for the Management of Patients With Peripheral Artery Disease (Updating the 2005 Guideline): A Report of the American College of Cardiology Foundation/American Heart Association Task Force on Practice guidelines. Circulation. 2011;124(18):2020-45.

147. Sanderson S, Emery J, Baglin T, Kinmonth AL. Narrative review: aspirin resistance and its clinical implications. Ann Intern Med. 2005;142(5):370-80. Epub 2005/03/02.

148. Dichiara J, Bliden KP, Tantry US, Chaganti SK, Kreutz RP, Gesheff TB, et al. Platelet function measured by VerifyNow (TM) identifies generalized high platelet reactivity in aspirin treated patients. Platelets. 2007;18(6):414-23.

149. McQuillan A, Eikelboom JW. Cyclooxygenase inhibitors and the antiplatelet effects of aspirin. New England Journal of Medicine. 2002;346(20):1589-.

150. Hennekens CH, Schneider WR, Hebert PR, Tantry US, Gurbel PA. Hypothesis formulation from subgroup analyses: Nonadherence or nonsteroidal anti-inflammatory drug use explains the lack of clinical benefit of aspirin on first myocardial infarction attributed to "aspirin resistance". Am Heart J. 2010;159(5):744-8.

151. Goodman T, Ferro A, Sharma P. Pharmacogenetics of aspirin resistance: a comprehensive systematic review. Brit J Clin Pharmaco. 2008;66(2):222-32.

152. Kuliczkowski W, Witkowski A, Polonski L, Watala C, Filipiak K, Budaj A, et al. Interindividual variability in the response to oral antiplatelet drugs: a position paper of the Working Group on antiplatelet drugs resistance appointed by the Section of Cardiovascular Interventions of the Polish Cardiac Society, endorsed by the Working Group on Thrombosis of the European Society of Cardiology. European Heart Journal. 2009;30(4):426-35.

153. Rajagopalan S, Ford I, Bachoo P, Hillis GS, Croal B, Greaves M, et al. Platelet activation, myocardial ischemic events and postoperative non-response to aspirin in patients undergoing major vascular surgery. J Thromb Haemost. 2007;5(10):2028-35. Epub 2007/07/26.

154. Calderaro D, Pastana AF, da Rocha TRF, Yu PC, Gualandro DM, DeLuccia N, et al. Aspirin responsiveness safely lowers perioperative cardiovascular risk. J Vasc Surg. 2013;58(6):1593-9.

155. Coller BS. Foreword - A Brief History of Ideas About Platelets in Health and Disease. Platelets (Third Edition): Academic Press; 2013. p. xix-xliv.

156. Schomig A, Neumann FJ, Kastrati A, Schuhlen H, Blasini R, Hadamitzky M, et al. A randomized comparison of antiplatelet and anticoagulant therapy after the placement of coronary-artery stents. $\mathrm{N}$ Engl J Med. 1996;334(17):1084-9. Epub 1996/04/25.

157. Cattaneo M. Platelet P2 receptors: old and new targets for antithrombotic drugs. Expert Rev Cardiovasc Ther. 2007;5(1):45-55. Epub 2006/12/26.

158. Bennett CL, Weinberg PD, Rozenberg-Ben-Dror K, Yarnold PR, Kwaan HC, Green D. Thrombotic thrombocytopenic purpura associated with ticlopidine. A review of 60 cases. Ann Intern Med. 1998;128(7):541-4. Epub 1998/06/10.

159. Balamuthusamy S, Arora R. Hematologic adverse effects of clopidogrel. Am J Ther. 2007;14(1):106-12. Epub 2007/02/17.

160. Raju NC, Eikelboom JW, Hirsh J. Platelet ADP-receptor antagonists for cardiovascular disease: past, present and future. Nat Clin Pract Cardiovasc Med. 2008;5(12):766-80. Epub 2008/10/30.

161. Kazui M, Nishiya Y, Ishizuka T, Hagihara K, Farid NA, Okazaki O, et al. Identification of the Human Cytochrome P450 Enzymes Involved in the Two Oxidative Steps in the Bioactivation of Clopidogrel to Its Pharmacologically Active Metabolite. Drug Metab Dispos. 2010;38(1):92-9. 
162. Bhatt DL, Flather MD, Hacke W, Berger PB, Black HR, Boden WE, et al. Patients with prior myocardial infarction, stroke, or symptomatic peripheral arterial disease in the CHARISMA trial. J Am Coll Cardiol. 2007;49(19):1982-8.

163. Wang TH, Bhatt DL, Fox KAA, Steinhubl SR, Brennan DM, Hacke W, et al. An analysis of mortality rates with dual-antiplatelet therapy in the primary prevention population of the CHARISMA trial. European Heart Journal. 2007;28(18):2200-7.

164. Cacoub PP, Bhatt DL, Steg PG, Topol EJ, Creager MA. Patients with peripheral arterial disease in the CHARISMA trial. European Heart Journal. 2009;30(2):192-201. Epub 2009/01/13.

165. Hankey GJ, Sudlow CL, Dunbabin DW. Thienopyridine derivatives (ticlopidine, clopidogrel) versus aspirin for preventing stroke and other serious vascular events in high vascular risk patients. Cochrane Database Syst Rev. 2000(2):CD001246. Epub 2000/05/05.

166. Diener HC, Bogousslavsky J, Brass LM, Cimminiello C, Csiba L, Kaste M, et al. Aspirin and clopidogrel compared with clopidogrel alone after recent ischaemic stroke or transient ischaemic attack in high-risk patients (MATCH): randomised, double-blind, placebo-controlled trial. Lancet. 2004;364(9431):331-7. Epub 2004/07/28.

167. Yusuf S, Fox KAA, Tognoni G, Mehta SR, Chrolavicius S, Anand S, et al. Effects of clopidogrel in addition to aspirin in patients with acute coronary syndromes without ST-segment elevation. New England Journal of Medicine. 2001;345(7):494-502.

168. Sabatine MS, Cannon CP, Gibson CM, Lopez-Sendon JL, Montalescot G, Theroux P, et al. Addition of clopidogrel to aspirin and fibrinolytic therapy for myocardial infarction with ST-segment elevation. New England Journal of Medicine. 2005;352(12):1179-89.

169. Chen ZM, Jiang LX, Chen YP, Xie JX, Pan HC, Peto R, et al. Addition of clopidogrel to aspirin in 45,852 patients with acute myocardial infarction: randomised placebo-controlled trial. Lancet. 2005;366(9497):1607-21.

170. Mehta SR, Yusuf S, Peters RJG, Bertrand ME, Lewis BS, Natarajan MK, et al. Effects of pretreatment with clopidogrel and aspirin followed by long-term therapy in patients undergoing percutaneous coronary intervention: the PCI-CURE study. The Lancet. 2001;358(9281):527-33.

171. Steinhubl SR, Berger PB, Mann IJ, et al. Early and sustained dual oral antiplatelet therapy following percutaneous coronary intervention: A randomized controlled trial. Jama. 2002;288(19):2411-20.

172. Sabatine MS, Cannon CP, Gibson CM, Lopez-Sendon JL, Montalescot G, Theroux P, et al. Effect of clopidogrel pretreatment before percutaneous coronary intervention in patients with ST-elevation myocardial infarction treated with fibrinolytics - The PCI-CLARITY study. Jama-J Am Med Assoc. 2005;294(10):1224-32.

173. King IIISB, Smith JSC, Hirshfeld JJW, Jacobs AK, Morrison DA, Williams DO. 2007 Focused Update of the ACC/AHA/SCAI 2005 Guideline Update for Percutaneous Coronary Intervention. J Am Coll Cardiol. 2008;51(2):172-209.

174. Effects of Clopidogrel in Addition to Aspirin in Patients with Acute Coronary Syndromes without ST-Segment Elevation. New England Journal of Medicine. 2001;345(7):494-502.

175. Jaremo P, Lindahl TL, Fransson SG, Richter A. Individual variations of platelet inhibition after loading doses of clopidogrel. J Intern Med. 2002;252(3):233-8.

176. Gurbel PA, Bliden KP, Hiatt BL, O'Connor CM. Clopidogrel for coronary stenting - Response variability, drug resistance, and the effect of pretreatment platelet reactivity. Circulation. 2003;107(23):2908-13.

177. Matetzky S, Shenkman B, Guetta V, Schechter M, Beinart R. Clopidogrel Resistance is Associated With Increased Risk of Recurrent Atherothrombotic Events in Patients With Acute Myocardial Infarction (vol 109, pg 3171, 2004). Circulation. 2011;124(17):E459-E.

178. Angiolillo DJ, Fernandez-Ortiz A, Bernardo E, Alfonso F, Macaya C, Bass TA, et al. Variability in individual responsiveness to clopidogrel: clinical implications, management, and future perspectives. J Am Coll Cardiol. 2007;49(14):1505-16. Epub 2007/04/10.

179. Bonello L, Tantry US, Marcucci R, Blindt R, Angiolillo DJ, Becker R, et al. Consensus and Future Directions on the Definition of High On-Treatment Platelet Reactivity to Adenosine Diphosphate. J Am Coll Cardiol. 2010;56(12):919-33.

180. Samara WM, Bliden KP, Tantry US, Gurbel PA. The difference between clopidogrel responsiveness and posttreatment platelet reactivity. Thrombosis Research. 2005;115(1-2):89-94. 
181. Gurbel PA, Bliden KP, Guyer K, Cho PW, Zaman KA, Kreutz RP, et al. Platelet reactivity in patients and recurrent events post-stenting: results of the PREPARE POST-STENTING Study. J Am Coll Cardiol. 2005;46(10):1820-6. Epub 2005/11/16.

182. Price MJ, Endemann S, Gollapudi RR, Valencia R, Stinis CT, Levisay JP, et al. Prognostic significance of post-clopidogrel platelet reactivity assessed by a point-of-care assay on thrombotic events after drug-eluting stent implantation. European Heart Journal. 2008;29(8):992-1000. Epub 2008/02/12.

183. Sibbing D, Braun S, Morath T, Mehilli J, Vogt W, Sch^mig A, et al. Platelet Reactivity After Clopidogrel Treatment Assessed With Point-of-Care Analysis and Early Drug-Eluting Stent Thrombosis. J Am Coll Cardiol. 2009;53(10):849-56.

184. Linnemann B, Schwonberg J, Toennes SW, Mani H, Lindhoff-Last E. Variability of residual platelet function despite clopidogrel treatment in patients with peripheral arterial occlusive disease. Atherosclerosis. 2010;209(2):504-9.

185. Barragan P, Bouvier JL, Roquebert PO, Macaluso G, Commeau P, Comet B, et al. Resistance to thienopyridines: clinical detection of coronary stent thrombosis by monitoring of vasodilatorstimulated phosphoprotein phosphorylation. Catheter Cardiovasc Interv. 2003;59(3):295-302. Epub 2003/06/25.

186. Matetzky S, Shenkman B, Guetta V, Schechter M, Bienart R, Goldenberg I, et al. Clopidogrel resistance is associated with increased risk of recurrent atherothrombotic events in patients with acute myocardial infarction. Circulation. 2004;109(25):3171-5.

187. Bliden KP, DiChiara J, Tantry US, Bassi AK, Chaganti SK, Gurbel PA. Increased risk in patients with high platelet aggregation receiving chronic clopidogrel therapy undergoing percutaneous coronary intervention: is the current antiplatelet therapy adequate? J Am Coll Cardiol. 2007;49(6):657-66. Epub 2007/02/13.

188. Blindt R, Stellbrink K, de Taeye A, Muller R, Kiefer P, Yagmur E, et al. The significance of vasodilator-stimulated phosphoprotein for risk stratification of stent thrombosis. Thromb Haemost. 2007;98(6):1329-34. Epub 2007/12/08.

189. Cuisset T Fau - Frere C, Frere C Fau - Quilici J, Quilici J Fau - Morange P-E, Morange Pe Fau Nait-Saidi L, Nait-Saidi L Fau - Mielot C, Mielot C Fau - Bali L, et al. High post-treatment platelet reactivity is associated with a high incidence of myonecrosis after stenting for non-ST elevation acute coronary syndromes. (0340-6245 (Print)).

190. Frere C, Cuisset T, Quilici J, Camoin L, Carvajal J, Morange PE, et al. ADP-induced platelet aggregation and platelet reactivity index VASP are good predictive markers for clinical outcomes in nonST elevation acute coronary syndrome. Thromb Haemost. 2007;98(4):838-43. Epub 2007/10/17.

191. Geisler T, Grass D, Bigalke B, Stellos K, Drosch T, Dietz K, et al. The Residual Platelet Aggregation after Deployment of Intracoronary Stent (PREDICT) score. J Thromb Haemost. 2008;6(1):54-61. Epub 2007/10/24.

192. Gurbel PA, Antonino MJ, Bliden KP, Dichiara J, Suarez TA, Singla A, et al. Platelet reactivity to adenosine diphosphate and long-term ischemic event occurrence following percutaneous coronary intervention: a potential antiplatelet therapeutic target. Platelets. 2008;19(8):595-604. Epub 2008/11/18.

193. Bonello L, Paganelli F, Arpin-Bornet M, Auquier P, Sampol J, Dignat-George F, et al. Vasodilatorstimulated phosphoprotein phosphorylation analysis prior to percutaneous coronary intervention for exclusion of postprocedural major adverse cardiovascular events. J Thromb Haemost. 2007;5(8):16306. Epub 2007/05/10.

194. Marcucci R, Gori AM, Paniccia R, Giusti B, Valente S, Giglioli C, et al. Cardiovascular death and nonfatal myocardial infarction in acute coronary syndrome patients receiving coronary stenting are predicted by residual platelet reactivity to ADP detected by a point-of-care assay: a 12-month followup. Circulation. 2009;119(2):237-42. Epub 2009/01/02.

195. Patti G, Nusca A, Mangiacapra F, Gatto L, D'Ambrosio A, Di Sciascio G. Point-of-care measurement of clopidogrel responsiveness predicts clinical outcome in patients undergoing percutaneous coronary intervention results of the ARMYDA-PRO (Antiplatelet therapy for Reduction of MYocardial Damage during Angioplasty-Platelet Reactivity Predicts Outcome) study. J Am Coll Cardiol. 2008;52(14):1128-33. Epub 2008/09/23.

196. Yusuf S, Zhao F, Mehta SR, Chrolavicius S, Tognoni G, Fox KK. Effects of clopidogrel in addition to aspirin in patients with acute coronary syndromes without ST-segment elevation. N Engl J Med. 2001;345(7):494-502. Epub 2001/08/25. 
197. Sibbing D, Schulz S, Braun S, Morath T, Stegherr J, Mehilli J, et al. Antiplatelet effects of clopidogrel and bleeding in patients undergoing coronary stent placement. Journal of Thrombosis and Haemostasis. 2010;8(2):250-6.

198. Campo G, Parrinello G, Ferraresi P, Lunghi B, Tebaldi M, Miccoli M, et al. Prospective Evaluation of On-Clopidogrel Platelet Reactivity Over Time in Patients Treated With Percutaneous Coronary Intervention Relationship With Gene Polymorphisms and Clinical Outcome. J Am Coll Cardiol. 2011;57(25):2474-83.

199. Rehmel JLF, Eckstein JA, Farid NA, Heim JB, Kasper SC, Kurihara A, et al. Interactions of two major metabolites of prasugrel, a thienopyridine antiplatelet agent, with the cytochromes P450. Drug Metab Dispos. 2006;34(4):600-7.

200. Niitsu Y, Jakubowski JA, Sugidachi A, Asai F. Pharmacology of CS-747 (prasugrel, LY640315), a novel, potent antiplatelet agent with in vivo P2Y12 receptor antagonist activity. Semin Thromb Hemost. 2005;31(2):184-94. Epub 2005/04/27.

201. Sugidachi A, Ogawa T, Kurihara A, Hagihara K, Jakubowski JA, Hashimoto M, et al. The greater in vivo antiplatelet effects of prasugrel as compared to clopidogrel reflect more efficient generation of its active metabolite with similar antiplatelet activity to that of clopidogrel's active metabolite. Journal of Thrombosis and Haemostasis. 2007;5(7):1545-51.

202. Brandt JT, Payne CD, Wiviott SD, Weerakkody G, Farid NA, Small DS, et al. A comparison of prasugrel and clopidogrel loading doses on platelet function: magnitude of platelet inhibition is related to active metabolite formation. Am Heart J. 2007;153(1).

203. Michelson AD, Frelinger AL, Braunwald E, Downey WE, Angiolillo DJ, Xenopoulos NP, et al. Pharmacodynamic assessment of platelet inhibition by prasugrel vs. clopidogrel in the TRITON-TIMI 38 trial. European Heart Journal. 2009;30(14):1753-63.

204. Jernberg T, Payne CD, Winters KJ, Darstein C, Brandt JT, Jakubowski JA, et al. Prasugrel achieves greater inhibition of platelet aggregation and a lower rate of non-responders compared with clopidogrel in aspirin-treated patients with stable coronary artery disease. European Heart Journal. 2006;27(10):1166-73. Epub 2006/04/20.

205. Small DS, Li YG, Ernest CS, April JH, Farid NA, Payne CD, et al. Integrated Analysis of Pharmacokinetic Data Across Multiple Clinical Pharmacology Studies of Prasugrel, a New Thienopyridine Antiplatelet Agent. J Clin Pharmacol. 2011;51(3):321-32.

206. Wiviott SD, Braunwald E, McCabe CH, Montalescot G, Ruzyllo W, Gottlieb S, et al. Prasugrel versus clopidogrel in patients with acute coronary syndromes. New England Journal of Medicine. 2007;357(20):2001-15.

207. Antman EM, Wiviott SD, Murphy SA, Voitk J, Hasin Y, Widimsky P, et al. Early and late benefits of prasugrel in patients with acute coronary syndromes undergoing percutaneous coronary intervention. J Am Coll Cardiol. 2008;51(21):2028-33.

208. Jakubowski JA, Riesmeyer JS, Close SL, Leishman AG, Erlinge D. TRITON and Beyond: New Insights into the Profile of Prasugrel. Cardiovasc Ther. 2012;30(4):e174-e82.

209. Wiviott SD, Braunwald E, Angiolillo DJ, Meisel S, Dalby AJ, Verheugt FWA, et al. Greater clinical benefit of more intensive oral antiplatelet therapy with prasugrel in patients with diabetes mellitus in the Trial to Assess Improvement in Therapeutic Outcomes by Optimizing Platelet Inhibition With Prasugrel-Thrombolysis in Myocardial Infarction 38. Circulation. 2008;118(16):1626-36.

210. Montalescot G, Wiviott SD, Brounwald E, Murphy SA, Gibson CM, McCabe CH, et al. Prasugrel compared with clopidogrel in patients undergoing percutaneous coronary intervention for ST-elevation myocardial infarction (TRITON-TIMI 38): double-blind, randomised controlled trial. Lancet. 2009;373(9665):723-31.

211. Murphy SA, Antman EM, Wiviott SD, Weerakkody G, Morocutti G, Huber K, et al. Reduction in recurrent cardiovascular events with prasugrel compared with clopidogrel in patients with acute coronary syndromes from the TRITON-TIMI 38 trial. European Heart Journal. 2008;29(20):2473-9.

212. Ajani $A E$, Lefkovits J. Approaching an age of reason with antiplatelet therapy. Lancet. 2008;371(9621):1315-6.

213. JJ VANG, Nilsson L, Berntsson P, Wissing BM, Giordanetto F, Tomlinson W, et al. Ticagrelor binds to human P2Y(12) independently from ADP but antagonizes ADP-induced receptor signaling and platelet aggregation. J Thromb Haemost. 2009;7(9):1556-65. Epub 2009/06/26.

214. Husted S, Emanuelsson H, Heptinstall S, Sandset PM, Wickens M, Peters G. Pharmacodynamics, pharmacokinetics, and safety of the oral reversible P2Y12 antagonist AZD6140 
with aspirin in patients with atherosclerosis: a double-blind comparison to clopidogrel with aspirin. European Heart Journal. 2006;27(9):1038-47. Epub 2006/02/16.

215. Gurbel PA, Bliden KP, Butler K, Tantry US, Gesheff T, Wei C, et al. Randomized double-blind assessment of the ONSET and OFFSET of the antiplatelet effects of ticagrelor versus clopidogrel in patients with stable coronary artery disease: the ONSET/OFFSET study. Circulation. 2009;120(25):257785. Epub 2009/11/20.

216. Cannon CP, Husted S, Harrington RA, Scirica BM, Emanuelsson H, Peters G, et al. Safety, tolerability, and initial efficacy of AZD6140, the first reversible oral adenosine diphosphate receptor antagonist, compared with clopidogrel, in patients with non-ST-segment elevation acute coronary syndrome: primary results of the DISPERSE-2 trial (vol 50, pg 1844, 2007). J Am Coll Cardiol. 2007;50(22):2196-.

217. Wallentin L, Becker RC, Budaj A, Cannon CP, Emanuelsson H, Held C, et al. Ticagrelor versus Clopidogrel in Patients with Acute Coronary Syndromes. New England Journal of Medicine. 2009;361(11):1045-57.

218. Becker RC, Bassand JP, Budaj A, Wojdyla DM, James SK, Cornel JH, et al. Bleeding complications with the P2Y12 receptor antagonists clopidogrel and ticagrelor in the PLATelet inhibition and patient Outcomes (PLATO) trial (vol 32, pg 2933, 2011). European Heart Journal. 2012;33(21):2750-.

219. Held C, Asenblad N, Bassand JP, Becker RC, Cannon CP, Claeys MJ, et al. Ticagrelor Versus Clopidogrel in Patients With Acute Coronary Syndromes Undergoing Coronary Artery Bypass Surgery Results From the PLATO (Platelet Inhibition and Patient Outcomes) Trial. J Am Coll Cardiol. 2011;57(6):672-84.

220. Harrington RA, Stone GW, McNulty S, White HD, Lincoff AM, Gibson CM, et al. Platelet inhibition with cangrelor in patients undergoing PCl. N Engl J Med. 2009;361(24):2318-29. Epub 2009/11/17.

221. Bhatt DL, Lincoff AM, Gibson CM, Stone GW, McNulty S, Montalescot G, et al. Intravenous platelet blockade with cangrelor during PCI. N Engl J Med. 2009;361(24):2330-41. Epub 2009/11/17.

222. Leonardi S, Rao SV, Harrington RA, Bhatt DL, Gibson CM, Roe MT, et al. Rationale and design of the randomized, double-blind trial testing INtraveNous and Oral administration of elinogrel, a selective and reversible P2Y(12)-receptor inhibitor, versus clopidogrel to eVAluate Tolerability and Efficacy in nonurgent Percutaneous Coronary Interventions patients (INNOVATE-PCI). Am Heart J. 2010;160(1):65-72. Epub 2010/07/06.

223. Angiolillo DJ, Capranzano P, Goto S, Aslam M, Desai B, Charlton RK, et al. A randomized study assessing the impact of cilostazol on platelet function profiles in patients with diabetes mellitus and coronary artery disease on dual antiplatelet therapy: results of the OPTIMUS-2 study. European Heart Journal. 2008;29(18):2202-11. Epub 2008/06/24.

224. Angiolillo DJ, Capodanno D, Goto S. Platelet thrombin receptor antagonism and atherothrombosis. European Heart Journal. 2010;31(1):17-28. Epub 2009/12/02.

225. Angiolillo DJ, Capranzano P, Desai B, Shoemaker SB, Charlton R, Zenni MM, et al. Impact of P2Y(12) inhibitory effects induced by clopidogrel on platelet procoagulant activity in type 2 diabetes mellitus patients. Thrombosis Research. 2009;124(3):318-22. Epub 2008/11/18.

226. Munch G, Ungerer M, Rosport K, Bultmann A, Piechatzek R, Uhland K, et al. Novel Antiplatelet Drug Revacept (Dimeric Glycoprotein VI-Fc) Specifically and Efficiently Inhibited Collagen-Induced Platelet Aggregation Without Affecting General Hemostasis in Humans. Circulation. 2011;123(17):1891-+.

227. Scott SA, Sangkuhl K, Gardner EE, Stein CM, Hulot JS, Johnson JA, et al. Clinical Pharmacogenetics Implementation Consortium Guidelines for Cytochrome P450-2C19 (CYP2C19) Genotype and Clopidogrel Therapy. Clinical Pharmacology \& Therapeutics. 2011;90(2):328-32.

228. Shuldiner AR, O'Connell JR, Bliden KP, Gandhi A, Ryan K, Horenstein RB, et al. Association of Cytochrome P450 2C19 Genotype With the Antiplatelet Effect and Clinical Efficacy of Clopidogrel Therapy. Jama-J Am Med Assoc. 2009;302(8):849-58.

229. Lea RA, Roberts RL, Green MR, Kennedy MA, Chambers GK. Allele frequency differences of cytochrome P450 polymorphisms in a sample of New Zealand Maori. N Z Med J. 2008;121(1272):33-7. Epub 2008/04/22.

230. Brandt JT, Close SL, Iturria SJ, Payne CD, Farid NA, Ernest CS, et al. Common polymorphisms of CYP2C19 and CYP2C9 affect the pharmacokinetic and pharmacodynamic response to clopidogrel but not prasugrel. Journal of Thrombosis and Haemostasis. 2007;5(12):2429-36. 
231. Gurbel PA, Shuldiner AR, Bliden KP, Ryan K, Pakyz RE, Tantry US. The relation between CYP2C19 genotype and phenotype in stented patients on maintenance dual antiplatelet therapy. Am Heart J. 2011;161(3):598-604.

232. Mega JL, Simon T, Collet JP, Anderson JL, Antman EM, Bliden K, et al. Reduced-Function CYP2C19 Genotype and Risk of Adverse Clinical Outcomes Among Patients Treated With Clopidogrel Predominantly for PCI A Meta-analysis. Jama-J Am Med Assoc. 2010;304(16):1821-30.

233. Hulot JS, Collet JP, Silvain J, Pena A, Bellemain-Appaix A, Barthelemy O, et al. Cardiovascular Risk in Clopidogrel-Treated Patients According to Cytochrome P450 2C19*2 Loss-of-Function Allele or Proton Pump Inhibitor Coadministration A Systematic Meta-Analysis. J Am Coll Cardiol. 2010;56(2):13443.

234. Holmes MV, Perel P, Shah T, Hingorani AD, Casas JP. CYP2C19 Genotype, Clopidogrel Metabolism, Platelet Function, and Cardiovascular Events A Systematic Review and Meta-analysis. Jama-J Am Med Assoc. 2011;306(24):2704-14.

235. Bauer T, Bouman HJ, van Werkum JW, Ford NF, ten Berg JM, Taubert D. Impact of CYP2C19 variant genotypes on clinical efficacy of antiplatelet treatment with clopidogrel: systematic review and meta-analysis. Brit Med J. 2011;343.

236. Bouman HJ, Harmsze AM, van Werkum JW, Breet NJ, Bergmeijer TO, Ten Cate $H$, et al. Variability in on-treatment platelet reactivity explained by CYP2C19*2 genotype is modest in clopidogrel pretreated patients undergoing coronary stenting. Heart. 2011;97(15):1239-44. Epub 2011/06/02.

237. Sibbing D, Koch W, Gebhard D, Schuster T, Braun S, Stegherr J, et al. Cytochrome 2C19*17 allelic variant, platelet aggregation, bleeding events, and stent thrombosis in clopidogrel-treated patients with coronary stent placement. Circulation. 2010;121(4):512-8. Epub 2010/01/20.

238. Bonello L, Tantry US, Marcucci R, Blindt R, Angiolillo DJ, Becker R, et al. Consensus and future directions on the definition of high on-treatment platelet reactivity to adenosine diphosphate. J Am Coll Cardiol. 2010;56(12):919-33. Epub 2010/09/11.

239. Bouman HJ, Schomig E, van Werkum JW, Velder J, Hackeng CM, Hirschhauser C, et al. Paraoxonase-1 is a major determinant of clopidogrel efficacy. Nat Med. 2011;17(1):110-6. Epub 2010/12/21.

240. Cattaneo M. Response variability to clopidogrel: is tailored treatment, based on laboratory testing, the right solution? J Thromb Haemost. 2012;10(3):327-36. Epub 2012/01/10.

241. Gurbel PA, Antonino MJ, Tantry US. Recent developments in clopidogrel pharmacology and their relation to clinical outcomes. Expert Opinion on Drug Metabolism and Toxicology. 2009;5(8):9891004.

242. Gilard M, Arnaud B, Cornily JC, Le Gal G, Lacut K, Le Calvez G, et al. Influence of omeprazole on the antiplatelet action of clopidogrel associated with aspirin: the randomized, double-blind OCLA (Omeprazole CLopidogrel Aspirin) study. J Am Coll Cardiol. 2008;51(3):256-60. Epub 2008/01/22.

243. Siller-Matula JM, Spiel AO, Lang IM, Kreiner G, Christ G, Jilma B. Effects of pantoprazole and esomeprazole on platelet inhibition by clopidogrel. Am Heart J. 2009;157(1):148 e1-5. Epub 2008/12/17.

244. Tantry US, Kereiakes DJ, Gurbel PA. Clopidogrel and Proton Pump Inhibitors: Influence of Pharmacological Interactions on Clinical Outcomes and Mechanistic Explanations. JACC: Cardiovascular Interventions. 2011;4(4):365-80.

245. Haffner SM, Lehto S, Rönnemaa T, Pyörälä K, Laakso M. Mortality from Coronary Heart Disease in Subjects with Type 2 Diabetes and in Nondiabetic Subjects with and without Prior Myocardial Infarction. New England Journal of Medicine. 1998;339(4):229-34.

246. Luscher TF, Creager MA, Beckman JA, Cosentino F. Diabetes and vascular disease: pathophysiology, clinical consequences, and medical therapy: Part II. Circulation. 2003;108(13):165561. Epub 2003/10/01.

247. Vinik Al, Erbas T, Park TS, Nolan R, Pittenger GL. Platelet dysfunction in type 2 diabetes. Diabetes Care. 2001;24(8):1476-85. Epub 2001/07/27.

248. Ferreiro JL, Angiolillo DJ. Diabetes and antiplatelet therapy in acute coronary syndrome. Circulation. 2011;123(7):798-813. Epub 2011/02/24.

249. Natarajan A, Zaman AG, Marshall SM. Platelet hyperactivity in type 2 diabetes: role of antiplatelet agents. Diab Vasc Dis Res. 2008;5(2):138-44. Epub 2008/06/10.

250. Morel O, Kessler L, Ohlmann P, Bareiss P. Diabetes and the platelet: toward new therapeutic paradigms for diabetic atherothrombosis. Atherosclerosis. 2010;212(2):367-76. Epub 2010/04/17. 
251. Halushka PV, Rogers RC, Loadholt CB, Colwell JA. Increased Platelet Thromboxane Synthesis in Diabetes-Mellitus. J Lab Clin Med. 1981;97(1):87-96.

252. Thomas G, Skrinska V, Lucas FV, Schumacher OP. Platelet Glutathione and Thromboxane Synthesis in Diabetes. Diabetes. 1985;34(10):951-4.

253. Li Y, Woo V, Bose R. Platelet hyperactivity and abnormal Ca2+ homeostasis in diabetes mellitus. American Journal of Physiology - Heart and Circulatory Physiology. 2001;280(4):H1480-H9.

254. Rao AK, Vaidyula VR, Boden G. Platelet and monocyte activation by hyperglycemia and hyperinsulinemia in healthy subjects. Platelets. 2006;17(8):577-85.

255. Ferroni P, Basili S, Falco A, Davi G. Platelet activation in type 2 diabetes mellitus. J Thromb Haemost. 2004;2(8):1282-91. Epub 2004/08/12.

256. Winocour PD, Watala C, Perry DW, Kinloughrathbone RL. Decreased Platelet Membrane Fluidity Due to Glycation or Acetylation of Membrane-Proteins. Thromb Haemost. 1992;68(5):577-82.

257. Colwell JA, Nesto RW. The Platelet in Diabetes. Diabetes Care. 2003;26(7):2181-8.

258. Martina V, Bruno GA, Trucco F, Zumpano E, Tagliabue M, Di Bisceglie C, et al. Platelet cNOS activity is reduced in patients with IDDM and NIDDM. Thromb Haemost. 1998;79(3):520-2.

259. Trovati M, Anfossi G, Massucco P, Mattiello L, Costamagna C, Piretto V, et al. Insulin stimulates nitric oxide synthesis in human platelets and, through nitric oxide, increases platelet concentrations of both guanosine-3',5'-cyclic monophosphate and adenosine-3',5'-cyclic monophosphate. Diabetes. 1997;46(5):742-9.

260. Westerbacka J, Yki-Jarvinen H, Turpeinen A, Rissanen A, Vehkavaara S, Syrjala M, et al. Inhibition of platelet-collagen interaction - An in vivo action of insulin abolished by insulin resistance in obesity. Arterioscl Throm Vas. 2002;22(1):167-72.

261. Akkerman JWN, Ferreira IA, Mocking AIM, Feijge MAH, Gorter G, van Haeften TW, et al. Platelet inhibition by insulin is absent in type 2 diabetes mellitus. Arterioscl Throm Vas. 2006;26(2):41722.

262. Matsuno H, Tokuda H, Ishisaki A, Zhou Y, Kitajima Y, Kozawa O. P2Y12 receptors play a significant role in the development of platelet microaggregation in patients with diabetes. J Clin Endocr Metab. 2005;90(2):920-7.

263. Ueno M, Ferreiro JL, Tomasello SD, Capodanno D, Tello-Montoliu A, Kodali M, et al. Functional profile of the platelet $\mathrm{P} 2 \mathrm{Y}$ receptor signalling pathway in patients with type 2 diabetes mellitus and coronary artery disease. Thromb Haemost. 2011;105(4):730-2. Epub 2011/01/13.

264. DiMinno G, Silver MJ, Cerbone AM, Murphy S. Trial of repeated low-dose aspirin in diabetic angiopathy. Blood. 1986;68(4):886-91. Epub 1986/10/01.

265. Guthikonda S, Alviar CL, Vaduganathan M, Arikan M, Tellez A, DeLao T, et al. Role of reticulated platelets and platelet size heterogeneity on platelet activity after dual antiplatelet therapy with aspirin and clopidogrel in patients with stable coronary artery disease. J Am Coll Cardiol. 2008;52(9):743-9. Epub 2008/08/23.

266. Guthikonda S, Lev El, Patel R, DeLao T, Bergeron AL, Dong JF, et al. Reticulated platelets and uninhibited COX-1 and COX-2 decrease the antiplatelet effects of aspirin. J Thromb Haemost. 2007;5(3):490-6. Epub 2007/02/27.

267. Sibbing D, von Beckerath O, Schomig A, Kastrati A, von Beckerath N. Impact of body mass index on platelet aggregation after administration of a high loading dose of $600 \mathrm{mg}$ of clopidogrel before percutaneous coronary intervention. Am J Cardiol. 2007;100(2):203-5. Epub 2007/07/17.

268. Bonello-Palot N, Armero S, Paganelli F, Mancini J, De Labriolle A, Bonello C, et al. Relation of body mass index to high on-treatment platelet reactivity and of failed clopidogrel dose adjustment according to platelet reactivity monitoring in patients undergoing percutaneous coronary intervention. Am J Cardiol. 2009;104(11):1511-5. Epub 2009/11/26.

269. Best PJ, Steinhubl SR, Berger PB, Dasgupta A, Brennan DM, Szczech LA, et al. The efficacy and safety of short- and long-term dual antiplatelet therapy in patients with mild or moderate chronic kidney disease: results from the Clopidogrel for the Reduction of Events During Observation (CREDO) trial. Am Heart J. 2008;155(4):687-93. Epub 2008/03/29.

270. Angiolillo DJ, Bernardo E, Capodanno D, Vivas D, Sabate M, Ferreiro JL, et al. Impact of chronic kidney disease on platelet function profiles in diabetes mellitus patients with coronary artery disease taking dual antiplatelet therapy. J Am Coll Cardiol. 2010;55(11):1139-46. Epub 2010/03/13.

271. Muller C, Caillard S, Jesel L, El Ghannudi S, Ohlmann P, Sauleau E, et al. Association of estimated GFR with platelet inhibition in patients treated with clopidogrel. American journal of kidney diseases : the official journal of the National Kidney Foundation. 2012;59(6):777-85. Epub 2012/03/20. 
272. Shi R, Ge L, Zhou X, Ji W-J, Lu R-Y, Zhang Y-Y, et al. Decreased platelet miR-223 expression is associated with high on-clopidogrel platelet reactivity. Thrombosis Research. 2013;131(6):508-13.

273. Sibbing D, Byrne RA, Kastrati A. Role of Platelet Function Testing in Clinical Practice: Current Concepts and Future Perspectives. Curr Drug Targets. 2011. Epub 2011/07/02.

274. Gurbel PA, Bliden KP, Hiatt BL, O'Connor CM. Clopidogrel for coronary stenting: response variability, drug resistance, and the effect of pretreatment platelet reactivity. Circulation. 2003;107(23):2908-13. Epub 2003/06/11.

275. Cattaneo M, Hayward CP, Moffat KA, Pugliano MT, Liu Y, Michelson AD. Results of a worldwide survey on the assessment of platelet function by light transmission aggregometry: a report from the platelet physiology subcommittee of the SSC of the ISTH. J Thromb Haemost. 2009;7(6):1029. Epub 2009/05/09.

276. Gurbel PA, Becker RC, Mann KG, Steinhubl SR, Michelson AD. Platelet function monitoring in patients with coronary artery disease. J Am Coll Cardiol. 2007;50(19):1822-34. Epub 2007/11/06.

277. Sibbing D, Braun S, Morath T, Mehilli J, Vogt W, Schomig A, et al. Platelet reactivity after clopidogrel treatment assessed with point-of-care analysis and early drug-eluting stent thrombosis. J Am Coll Cardiol. 2009;53(10):849-56. Epub 2009/03/07.

278. Siller-Matula JM, Christ G, Lang IM, Delle-Karth G, Huber K, Jilma B. Multiple electrode aggregometry predicts stent thrombosis better than the vasodilator-stimulated phosphoprotein phosphorylation assay. Journal of Thrombosis and Haemostasis. 2010;8(2):351-9.

279. Aradi D, Komocsi A, Vorobcsuk A, Rideg O, Tokes-Fuzesi M, Magyarlaki T, et al. Prognostic significance of high on-clopidogrel platelet reactivity after percutaneous coronary intervention: systematic review and meta-analysis. Am Heart J. 2010;160(3):543-51. Epub 2010/09/10.

280. Wiviott SD, Braunwald E, McCabe CH, Montalescot G, Ruzyllo W, Gottlieb S, et al. Prasugrel versus clopidogrel in patients with acute coronary syndromes. N Engl J Med. 2007;357(20):2001-15. Epub 2007/11/06.

281. Sibbing D, Steinhubl SR, Schulz S, Schomig A, Kastrati A. Platelet aggregation and its association with stent thrombosis and bleeding in clopidogrel-treated patients: initial evidence of a therapeutic window. J Am Coll Cardiol. 2010;56(4):317-8. Epub 2010/07/17.

282. Gurbel PA, Tantry US. Chapter 30 - Monitoring of Antiplatelet Therapy. Platelets (Third Edition): Academic Press; 2013. p. 603-33.

283. Bonello L, Camoin-Jau L, Arques S, Boyer C, Panagides D, Wittenberg O, et al. Adjusted clopidogrel loading doses according to vasodilator-stimulated phosphoprotein phosphorylation index decrease rate of major adverse cardiovascular events in patients with clopidogrel resistance: a multicenter randomized prospective study. J Am Coll Cardiol. 2008;51(14):1404-11. Epub 2008/04/05. 284. Bonello L, Camoin-Jau L, Armero S, Com O, Arques S, Burignat-Bonello C, et al. Tailored clopidogrel loading dose according to platelet reactivity monitoring to prevent acute and subacute stent thrombosis. Am J Cardiol. 2009;103(1):5-10. Epub 2008/12/23.

285. Valgimigli M, Campo G, de Cesare N, Meliga E, Vranckx P, Furgieri A, et al. Intensifying platelet inhibition with tirofiban in poor responders to aspirin, clopidogrel, or both agents undergoing elective coronary intervention: results from the double-blind, prospective, randomized Tailoring Treatment with Tirofiban in Patients Showing Resistance to Aspirin and/or Resistance to Clopidogrel study. Circulation. 2009;119(25):3215-22. Epub 2009/06/17.

286. Price MJ. Standard versus High-Dose Clopidogrel According to Platelet Function Testing After PCI: Results of the GRAVITAS Trial. Circulation. 2010;122(21):2218-

287. Collet JP, Cuisset T, Range G, Cayla G, Elhadad S, Pouillot C, et al. Bedside monitoring to adjust antiplatelet therapy for coronary stenting. N Engl J Med. 2012;367(22):2100-9. Epub 2012/11/06.

288. Trenk D, Stone GW, Gawaz M, Kastrati A, Angiolillo DJ, Muller U, et al. A Randomized Trial of Prasugrel Versus Clopidogrel in Patients With High Platelet Reactivity on Clopidogrel After Elective Percutaneous Coronary Intervention With Implantation of Drug-Eluting Stents Results of the TRIGGERPCI (Testing Platelet Reactivity In Patients Undergoing Elective Stent Placement on Clopidogrel to Guide Alternative Therapy With Prasugrel) Study. J Am Coll Cardiol. 2012;59(24):2159-64.

289. Thygesen K, Alpert JS, Jaffe AS, Simoons ML, Chaitman BR, White HD, et al. Third universal definition of myocardial infarction. Circulation. 2012;126(16):2020-35. Epub 2012/08/28.

290. Paniccia R, Antonucci E, Maggini N, Romano E, Gori AM, Marcucci R, et al. Assessment of platelet function on whole blood by multiple electrode aggregometry in high-risk patients with coronary artery disease receiving antiplatelet therapy. Am J Clin Pathol. 2009;131(6):834-42. Epub 2009/05/23. 
291. Breugelmans J, Vertessen F, Mertens G, Gadisseur A, Van der Planken M. Multiplate whole blood impedance aggregometry: a recent experience. Thromb Haemost. 2008;100(4):725-6. Epub 2008/10/09.

292. Paniccia R, Antonucci E, Maggini N, Miranda M, Gori AM, Marcucci R, et al. Comparison of methods for monitoring residual platelet reactivity after clopidogrel by point-of-care tests on whole blood in high-risk patients. Thromb Haemost. 2010;104(2):287-92. Epub 2010/05/12.

293. Seyfert UT, Haubelt H, Vogt A, Hellstern P. Variables influencing Multiplate(TM) whole blood impedance platelet aggregometry and turbidimetric platelet aggregation in healthy individuals. Platelets. 2007;18(3):199-206. Epub 2007/05/15.

294. Sibbing D, Braun S, Jawansky S, Vogt W, Mehilli J, Schomig A, et al. Assessment of ADP-induced platelet aggregation with light transmission aggregometry and multiple electrode platelet aggregometry before and after clopidogrel treatment. Thromb Haemost. 2008;99(1):121-6. Epub 2008/01/25.

295. Toth O, Calatzis A, Penz S, Losonczy H, Siess W. Multiple electrode aggregometry: a new device to measure platelet aggregation in whole blood. Thromb Haemost. 2006;96(6):781-8. Epub 2006/12/02.

296. Paniccia R, Antonucci E, Gori AM, Marcucci R, Giglioli C, Antoniucci D, et al. Different methodologies for evaluating the effect of clopidogrel on platelet function in high-risk coronary artery disease patients. J Thromb Haemost. 2007;5(9):1839-47. Epub 2007/08/29.

297. Sibbing D, Schulz S, Braun S, Morath T, Stegherr J, Mehilli J, et al. Antiplatelet effects of clopidogrel and bleeding in patients undergoing coronary stent placement. J Thromb Haemost. 2010;8(2):250-6. Epub 2009/12/01.

298. Marciniak SJ, Jr., Jordan RE, Mascelli MA. Effect of Ca2+ chelation on the platelet inhibitory ability of the GPIIb/IIla antagonists abciximab, eptifibatide and tirofiban. Thromb Haemost. 2001;85(3):539-43. Epub 2001/04/20.

299. Mousa SA, Bozarth JM, Forsythe MS, Slee A. Differential antiplatelet efficacy for various GPIIb/IIla antagonists. Cardiovascular Research. 2000;47(4):819-26.

300. Kalb ML, Potura L, Scharbert G, Kozek-Langenecker SA. The effect of ex vivo anticoagulants on whole blood platelet aggregation. Platelets. 2009;20(1):7-11. Epub 2009/01/28.

301. Harding SA, Din JN, Sarma J, Josephs DH, Fox KAA, Newby DE. Promotion of proinflammatory interactions between platelets and monocytes by unfractionated heparin. Heart. 2006;92(11):1635-8.

302. Thomson C, Forbes CD, Prentice CR. The potentiation of platelet aggregation and adhesion by heparin in vitro and in vivo. Clin Sci Mol Med. 1973;45(4):485-94. Epub 1973/10/01.

303. Salzman EW, Rosenberg RD, Smith MH, Lindon JN, Favreau L. Effect of heparin and heparin fractions on platelet aggregation. J Clin Invest. 1980;65(1):64-73. Epub 1980/01/01.

304. Heinrich D, Gorg T, Schulz M. Effects of unfractionated and fractionated heparin on platelet function. Haemostasis. 1988;18 Suppl 3:48-54. Epub 1988/01/01.

305. Blakely T TM, Atkinson J, Yeh LC, Huang K. Tracking Disparity: Trends in ethnic and socioeconomic inequalities in mortality 1981-2004. In: Health Mo, editor. Wellington: Ministry of Health; 2007.

306. Mega JL, Simon T, Collet JP, Anderson JL, Antman EM, Bliden K, et al. Reduced-function CYP2C19 genotype and risk of adverse clinical outcomes among patients treated with clopidogrel predominantly for PCI: a meta-analysis. Jama. 2010;304(16):1821-30. Epub 2010/10/28.

307. Small DS, Farid NA, Payne CD, Konkoy CS, Jakubowski JA, Winters KJ, et al. Effect of intrinsic and extrinsic factors on the clinical pharmacokinetics and pharmacodynamics of prasugrel. Clin Pharmacokinet. 2010;49(12):777-98. Epub 2010/11/09.

308. Sibbing DS, S. R. Schulz, S. Schomig, A. Kastrati, A. Platelet Aggregation and Its Association With Stent Thrombosis and Bleeding in Clopidogrel-Treated Patients. J Am Coll Cardiol. 2010;56(4):317-8.

309. Hochholzer W, Trenk D, Fromm MF, Valina CM, Stratz C, Bestehorn HP, et al. Impact of cytochrome P450 2C19 loss-of-function polymorphism and of major demographic characteristics on residual platelet function after loading and maintenance treatment with clopidogrel in patients undergoing elective coronary stent placement. J Am Coll Cardiol. 2010;55(22):2427-34. Epub 2010/06/01.

310. Angiolillo DJ, Bernardo E, Sabate M, Jimenez-Quevedo P, Costa MA, Palazuelos J, et al. Impact of platelet reactivity on cardiovascular outcomes in patients with type 2 diabetes mellitus and coronary artery disease. J Am Coll Cardiol. 2007;50(16):1541-7. Epub 2007/10/16. 
311. Mangiacapra F, Patti G, Peace A, Gatto L, Vizzi V, Ricottini E, et al. Comparison of platelet reactivity and periprocedural outcomes in patients with versus without diabetes mellitus and treated with clopidogrel and percutaneous coronary intervention. Am J Cardiol. 2010;106(5):619-23. Epub 2010/08/21.

312. Angiolillo DJ, Shoemaker SB, Desai B, Yuan H, Charlton RK, Bernardo E, et al. Randomized comparison of a high clopidogrel maintenance dose in patients with diabetes mellitus and coronary artery disease: results of the Optimizing Antiplatelet Therapy in Diabetes Mellitus (OPTIMUS) study. Circulation. 2007;115(6):708-16. Epub 2007/01/31.

313. Wiviott SD, Braunwald E, Angiolillo DJ, Meisel S, Dalby AJ, Verheugt FW, et al. Greater clinical benefit of more intensive oral antiplatelet therapy with prasugrel in patients with diabetes mellitus in the trial to assess improvement in therapeutic outcomes by optimizing platelet inhibition with prasugrel-Thrombolysis in Myocardial Infarction 38. Circulation. 2008;118(16):1626-36. Epub 2008/09/02.

314. Roffi M, Chew DP, Mukherjee D, Bhatt DL, White JA, Heeschen C, et al. Platelet glycoprotein Ilb/IIla inhibitors reduce mortality in diabetic patients with non-ST-segment-elevation acute coronary syndromes. Circulation. 2001;104(23):2767-71. Epub 2001/12/06.

315. Tomlin A, Tilyard M, Dawson A, Dovey S. Health status of New Zealand European, Maori, and Pacific patients with diabetes at 242 New Zealand general practices. N Z Med J. 2006;119(1235):U2004. Epub 2006/06/06.

316. Patti G, Grieco D, Dicuonzo G, Pasceri V, Nusca A, Di Sciascio G. High versus standard clopidogrel maintenance dose after percutaneous coronary intervention and effects on platelet inhibition, endothelial function, and inflammation results of the ARMYDA-150 mg (antiplatelet therapy for reduction of myocardial damage during angioplasty) randomized study. J Am Coll Cardiol. 2011;57(7):771-8. Epub 2011/02/12.

317. Gurbel PA, Tantry US. An initial experiment with personalized antiplatelet therapy: the GRAVITAS trial. Jama. 2011;305(11):1136-7. Epub 2011/03/17.

318. Mehta SR, Tanguay JF, Eikelboom JW, Jolly SS, Joyner CD, Granger CB, et al. Double-dose versus standard-dose clopidogrel and high-dose versus low-dose aspirin in individuals undergoing percutaneous coronary intervention for acute coronary syndromes (CURRENT-OASIS 7): a randomised factorial trial. Lancet. 2010;376(9748):1233-43. Epub 2010/09/08.

319. Temesvari M, Paulik J, Kobori L, Monostory K. High-resolution melting curve analysis to establish CYP2C19 *2 single nucleotide polymorphism: Comparison with hydrolysis SNP analysis. Molecular and cellular probes. 2011;25(2-3):130-3. Epub 2011/02/15.

320. Cuisset T, Gaborit B, Dubois N, Quilici J, Loosveld M, Beguin S, et al. Platelet reactivity in diabetic patients undergoing coronary stenting for acute coronary syndrome treated with clopidogrel loading dose followed by prasugrel maintenance therapy. Int J Cardiol. 2013;168(1):523-8. Epub 2012/10/23.

321. Serebruany V, Pokov I, Kuliczkowski W, Chesebro J, Badimon J. Baseline platelet activity and response after clopidogrel in 257 diabetics among 822 patients with coronary artery disease. Thromb Haemost. 2008;100(1):76-82.

322. Morel O, Kessler L, OhImann P, Bareiss P. Diabetes and the platelet: Toward new therapeutic paradigms for diabetic atherothrombosis. Atherosclerosis. 2010;212(2):367-76.

323. Angiolillo DJ, Bernardo E, Sabate M, Jimenez-Quevedo P, Costa MA, Palazuelos J, et al. Impact of platelet reactivity on cardiovascular outcomes in patients with type 2 diabetes mellitus and coronary artery disease. J Am Coll Cardiol. 2007;50(16):1541-7.

324. Morel O Fau - El Ghannudi S, El Ghannudi S Fau - Hess S, Hess S Fau - Reydel A, Reydel A Fau Crimizade U, Crimizade U Fau - Jesel L, Jesel L Fau - Radulescu B, et al. The extent of P2Y12 inhibition by clopidogrel in diabetes mellitus patients with acute coronary syndrome is not related to glycaemic control: roles of white blood cell count and body weight. (0340-6245 (Print)).

325. Cesari F, Marcucci R, Caporale R, Paniccia R, Romano E, Gensin GF, et al. Relationship between high platelet turnover and platelet function in high-risk patients with coronary artery disease on dual antiplatelet therapy. Thromb Haemost. 2008;99(5):930-5.

326. Perl L, Lerman-Shivek H, Rechavia E, Vaduganathan $M$, Leshem-Lev D, Zemer-Wassercug $N$, et al. Response to Prasugrel and Levels of Circulating Reticulated Platelets in Patients with ST-Elevation Myocardial Infarction. J Am Coll Cardiol. 2013. Epub 2013/10/24. 
327. Sewell R, Ibbotson RM, Phillips R, Carson P. High mean platelet volume after myocardial infarction: is it due to consumption of small platelets? Br Med J (Clin Res Ed). 1984;289(6458):1576-8. Epub 1984/12/08.

328. Robinson MS, Mackie IJ, Khair K, Liesner R, Goodall AH, Savidge GF, et al. Flow cytometric analysis of reticulated platelets: evidence for a large proportion of non-specific labelling of dense granules by fluorescent dyes. Br J Haematol. 1998;100(2):351-7. Epub 1998/03/06.

329. Jakubowski JA, Thompson CB, Vaillancourt R, Valeri CR, Deykin D. Arachidonic acid metabolism by platelets of differing size. Br J Haematol. 1983;53(3):503-11. Epub 1983/03/01.

330. Martin JF, Trowbridge EA, Salmon G, Plumb J. The biological significance of platelet volume: its relationship to bleeding time, platelet thromboxane B2 production and megakaryocyte nuclear DNA concentration. Thromb Res. 1983;32(5):443-60. Epub 1983/12/01.

331. Stissing T, Dridi NP, Ostrowski SR, Bochsen L, Johansson PI. The Influence of Low Platelet Count on Whole Blood Aggregometry Assessed by Multiplate. Clin Appl Thromb-Hem. 2011;17(6):E211-E7.

332. Hanke AA, Roberg K, Monaca E, Sellmann T, Weber CF, Rahe-Meyer N, et al. Impact of Platelet Count on Results Obtained from Multiple Electrode Platelet Aggregometry (Multiplate (Tm)). Eur J Med Res. 2010;15(5):214-9.

333. Hsu HL, Woad KJ, Woodfield DG, Helsby NA. A high incidence of polymorphic CYP2C19 variants in archival blood samples from Papua New Guinea. Hum Genomics. 2008;3(1):17-23. Epub 2009/01/09. 334. Ruiter R, Bijl MJ, van Schaik RHN, Berns EMJJ, Hofman A, Coebergh JWW, et al. CYP2C19*2 polymorphism is associated with increased survival in breast cancer patients using tamoxifen. Pharmacogenomics. 2010;11(10):1367-75.

335. Brackbill ML, Kidd RS, Abdoo AD, Warner JG, Harralson AF. Frequency of CYP3A4, CYP3A5, CYP2C9, and CYP2C19 variant alleles in patients receiving clopidogrel that experience repeat acute coronary syndrome. Heart Vessels. 2009;24(2):73-8.

336. Goldstein JA, Ishizaki T, Chiba K, deMorais SMF, Bell D, Krahn PM, et al. Frequencies of the defective CYP2C19 alleles responsible for the mephenytoin poor metabolizer phenotype in various Oriental, Caucasian, Saudi Arabian and American black populations. Pharmacogenetics. 1997;7(1):5964.

337. Holmes DR, Jr., Dehmer GJ, Kaul S, Leifer D, O'Gara PT, Stein CM. ACCF/AHA Clopidogrel clinical alert: approaches to the FDA "boxed warning": a report of the American College of Cardiology Foundation Task Force on Clinical Expert Consensus Documents and the American Heart Association. Circulation. 2010;122(5):537-57. Epub 2010/06/30.

338. Patti G, Pasceri V, Vizzi V, Ricottini E, Di Sciascio G. Usefulness of Platelet Response to Clopidogrel by Point-of-Care Testing to Predict Bleeding Outcomes in Patients Undergoing Percutaneous Coronary Intervention (from the Antiplatelet Therapy for Reduction of Myocardial Damage During Angioplasty-Bleeding Study). American Journal of Cardiology. 2011;107(7):995-1000.

339. Cuisset T, Frere C, Quilici J, Morange PE, Mouret JP, Bali L, et al. Glycoprotein IIb/IIla Inhibitors Improve Outcome After Coronary Stenting in Clopidogrel Nonresponders A Prospective, Randomized Study. Jacc-Cardiovasc Inte. 2008;1(6):649-53.

340. Harmsze AM, van Werkum JW, Hackeng CM, Ruven HJT, Kelder JC, Bouman HJ, et al. The influence of CYP2C19*2 and*17 on on-treatment platelet reactivity and bleeding events in patients undergoing elective coronary stenting. Pharmacogenet Genom. 2012;22(3):169-75.

341. Kwak YL, Kim JC, Choi YS, Yoo KJ, Song Y, Shim JK. Clopidogrel Responsiveness Regardless of the Discontinuation Date Predicts Increased Blood Loss and Transfusion Requirement After Off-Pump Coronary Artery Bypass Graft Surgery. J Am Coll Cardiol. 2010;56(24):1994-2002.

342. Poston R, Gu JY, Manchio J, Lee A, Brown J, Gammie J, et al. Platelet function tests predict bleeding and thrombotic events after off-pump coronary bypass grafting. Eur J Cardio-Thorac. 2005;27(4):584-90.

343. Cuisset T, Quilici J, Loosveld M, Gaborit B, Grosdidier C, Fourcade L, et al. Comparison between initial and chronic response to clopidogrel therapy after coronary stenting for acute coronary syndrome and influence on clinical outcomes. Am Heart J. 2012;164(3):327-33.

344. Sibbing D, Braun S, Morath T, Mehilli J, Vogt W, Schomig A, et al. Platelet reactivity after clopidogrel treatment assessed with point-of-care analysis and early drug-eluting stent thrombosis. J Am Coll Cardiol. 2009;53(10):849-56. Epub 2009/03/07.

345. Cutlip DE, Windecker S, Mehran R, Boam A, Cohen DJ, van Es GA, et al. Clinical end points in coronary stent trials - A case for standardized definitions. Circulation. 2007;115(17):2344-51. 
346. Mehran R, Rao SV, Bhatt DL, Gibson CM, Caixeta A, Eikelboom J, et al. Standardized bleeding definitions for cardiovascular clinical trials: a consensus report from the Bleeding Academic Research Consortium. Circulation. 2011;123(23):2736-47. Epub 2011/06/15.

347. Matetzky S, Shenkman B, Guetta V, Shechter M, Bienart R, Goldenberg I, et al. Clopidogrel resistance is associated with increased risk of recurrent atherothrombotic events in patients with acute myocardial infarction. Circulation. 2004;109(25):3171-5. Epub 2004/06/09.

348. Eikelboom JW, Mehta SR, Anand SS, Xie C, Fox KA, Yusuf S. Adverse impact of bleeding on prognosis in patients with acute coronary syndromes. Circulation. 2006;114(8):774-82. Epub 2006/08/16.

349. Kinnaird TD, Stabile E, Mintz GS, Lee CW, Canos DA, Gevorkian N, et al. Incidence, predictors, and prognostic implications of bleeding and blood transfusion following percutaneous coronary interventions. Am J Cardiol. 2003;92(8):930-5. Epub 2003/10/15.

350. Kirtane AJ, Piazza G, Murphy SA, Budiu D, Morrow DA, Cohen DJ, et al. Correlates of bleeding events among moderate-to high-risk patients undergoing percutaneous coronary intervention and treated with eptifibatide - Observations from the PROTECT-TIMI-30 Trial. J Am Coll Cardiol. 2006;47(12):2374-9.

351. Manoukian SV, Feit F, Mehran R, Voeltz MD, Ebrahimi R, Hamon M, et al. Impact of major bleeding on 30-day mortality and clinical outcomes in patients with acute coronary syndromes - An analysis from the ACUITY trial. J Am Coll Cardiol. 2007;49(12):1362-8.

352. Moscucci M, Fox KA, Cannon CP, Klein W, Lopez-Sendon J, Montalescot G, et al. Predictors of major bleeding in acute coronary syndromes: the Global Registry of Acute Coronary Events (GRACE). Eur Heart J. 2003;24(20):1815-23. Epub 2003/10/18.

353. Cuisset T, Cayla G, Frere C, Quilici J, Poyet R, Gaborit B, et al. Predictive value of post-treatment platelet reactivity for occurrence of post-discharge bleeding after non-ST elevation acute coronary syndrome. Shifting from antiplatelet resistance to bleeding risk assessment? Eurointervention. 2009;5(3):325-9.

354. Gurbel PA, Bliden KP, Navickas IA, Mahla E, Dichiara J, Suarez TA, et al. Adenosine diphosphateinduced platelet-fibrin clot strength: a new thrombelastographic indicator of long-term poststenting ischemic events. Am Heart J. 2010;160(2):346-54. Epub 2010/08/10.

355. Mokhtar OA, Armero S, Tahirou I, Paganelli F, Bonello L, Lemesle G, et al. Relationship between platelet reactivity inhibition and non-CABG related major bleeding in patients undergoing percutaneous coronary intervention. Thrombosis Research. 2010;126(2):E147-E9.

356. Tsukahara K, Kimura K, Morita S, Ebina T, Kosuge M, Hibi K, et al. Impact of HighResponsiveness to Dual Antiplatelet Therapy on Bleeding Complications in Patients Receiving DrugEluting Stents. Circ J. 2010;74(4):679-85.

357. Price MJ, Berger PB, Teirstein PS, Tanguay JF, Angiolillo DJ, Spriggs D, et al. Standard- vs highdose clopidogrel based on platelet function testing after percutaneous coronary intervention: the GRAVITAS randomized trial. Jama. 2011;305(11):1097-105. Epub 2011/03/17.

358. Breet NJ, van Werkum JW, Bouman HJ, Kelder JC, Ruven HJ, Bal ET, et al. Comparison of platelet function tests in predicting clinical outcome in patients undergoing coronary stent implantation. Jama. 2010;303(8):754-62. Epub 2010/02/25.

359. Valgimigli M, Campo G, de Cesare N, Vranckx P, Hamon M, Angiolillo DJ, et al. Tailoring treatment with tirofiban in patients showing resistance to aspirin and/or resistance to clopidogrel (3T/2R). Rationale for the study and protocol design. Cardiovascular drugs and therapy / sponsored by the International Society of Cardiovascular Pharmacotherapy. 2008;22(4):313-20. Epub 2008/06/19.

360. Jernberg T, Payne CD, Winters KJ, Darstein C, Brandt JT, Jakubowski JA, et al. Prasugrel achieves greater inhibition of platelet aggregation and a lower rate of non-responders compared with clopidogrel in aspirin-treated patients with stable coronary artery disease. Eur Heart J. 2006;27(10):1166-73. Epub 2006/04/20.

361. Bliden KP, Tantry US, Storey RF, Jeong YH, Gesheff M, Wei C, et al. The effect of ticagrelor versus clopidogrel on high on-treatment platelet reactivity: combined analysis of the ONSET/OFFSET and RESPOND studies. Am Heart J. 2011;162(1):160-5. Epub 2011/07/12.

362. Stone GW, Witzenbichler B, Weisz G, Rinaldi MJ, Neumann FJ, Metzger DC, et al. Platelet reactivity and clinical outcomes after coronary artery implantation of drug-eluting stents (ADAPT-DES): a prospective multicentre registry study. Lancet. 2013. Epub 2013/07/31.

363. De Caterina R, Marchioli R, Andreotti F. Bedside monitoring of antiplatelet therapy for coronary stenting. N Engl J Med. 2013;368(9):871. Epub 2013/03/01. 
364. Price MJ, Murray SS, Angiolillo DJ, Lillie E, Smith EN, Tisch RL, et al. Influence of genetic polymorphisms on the effect of high- and standard-dose clopidogrel after percutaneous coronary intervention: the GIFT (Genotype Information and Functional Testing) study. J Am Coll Cardiol. 2012;59(22):1928-37. Epub 2012/05/26.

365. Becker RC, Bassand JP, Budaj A, Wojdyla DM, James SK, Cornel JH, et al. Bleeding complications with the P2Y12 receptor antagonists clopidogrel and ticagrelor in the PLATelet inhibition and patient Outcomes (PLATO) trial. Eur Heart J. 2011;32(23):2933-44. Epub 2011/11/18.

366. Ashton CM, Petersen NJ, Wray NP, Kiefe Cl, Dunn JK, Wu L, et al. The incidence of perioperative myocardial infarction in men undergoing noncardiac surgery. Ann Intern Med. 1993;118(7):504-10. Epub 1993/04/01.

367. Landesberg G, Shatz V, Akopnik I, Wolf YG, Mayer M, Berlatzky Y, et al. Association of cardiac troponin, $\mathrm{CK}-\mathrm{MB}$, and postoperative myocardial ischemia with long-term survival after major vascular surgery. J Am Coll Cardiol. 2003;42(9):1547-54. Epub 2003/11/11.

368. Landesberg G, Mosseri M, Shatz V, Akopnik I, Bocher M, Mayer M, et al. Cardiac troponin after major vascular surgery: the role of perioperative ischemia, preoperative thallium scanning, and coronary revascularization. J Am Coll Cardiol. 2004;44(3):569-75. Epub 2004/09/11.

369. Bursi F, Babuin L, Barbieri A, Politi L, Zennaro M, Grimaldi T, et al. Vascular surgery patients: perioperative and long-term risk according to the ACC/AHA guidelines, the additive role of postoperative troponin elevation. European Heart Journal. 2005;26(22):2448-56. Epub 2005/08/02.

370. Cabral HJ. Multiple Comparisons Procedures. Circulation. 2008;117(5):698-701.

371. Saunders J, Nambi V, Kimball KT, Virani SS, Morrisett JD, Lumsden AB, et al. Variability and persistence of aspirin response in lower extremity peripheral arterial disease patients. J Vasc Surg. 2011;53(3):668-75. Epub 2011/01/14.

372. van der Loo B, Braun J, Koppensteiner R. On-treatment function testing of platelets and longterm outcome of patients with peripheral arterial disease undergoing transluminal angioplasty. Eur J Vasc Endovasc Surg. 2011;42(6):809-16. Epub 2011/09/16.

373. Gualandro DM, Calderaro D, Caramelli B. Controversies regarding the pathophysiology of perioperative myocardial infarction. Catheter Cardio Inte. 2013;81(4):744-.

374. Gualandro DM, Campos CA, Calderaro D, Yu PC, Marques AC, Pastana AF, et al. Coronary plaque rupture in patients with myocardial infarction after noncardiac surgery: Frequent and dangerous. Atherosclerosis. 2012;222(1):191-5.

375. Tantry US, Bonello L, Aradi D, Price MJ, Jeong YH, Angiolillo DJ, et al. Consensus and update on the definition of on-treatment platelet reactivity to adenosine diphosphate associated with ischemia and bleeding. J Am Coll Cardiol. 2013;62(24):2261-73. Epub 2013/10/01.

376. Reny JL, Berdague P, Poncet A, Barazer I, Nolli S, Fabbro-Peray P, et al. Antiplatelet drug response status does not predict recurrent ischemic events in stable cardiovascular patients: results of the Antiplatelet Drug Resistances and Ischemic Events study. Circulation. 2012;125(25):3201-10. doi: 10.1161/CIRCULATIONAHA.111.085464. Epub 2012 May 21.

377. Burdess A, Nimmo AF, Campbell N, Harding SA, Garden OJ, Dawson AR, et al. Perioperative platelet and monocyte activation in patients with critical limb ischemia. J Vasc Surg. 2010;52(3):697703. Epub 2010/09/08.

378. Apple FS, Ler R, Murakami MM. Determination of 19 cardiac troponin I and T assay 99th percentile values from a common presumably healthy population. Clinical chemistry. 2012;58(11):1574-81. Epub 2012/09/18.

379. Melanson SEF, Morrow DA, Jarolim P. Earlier detection of myocardial injury in a preliminary evaluation using a new troponin I assay with improved sensitivity. Am J Clin Pathol. 2007;128(2):282-6. 380. Chenevier-Gobeaux C, Meune C, Freund Y, Wahbi K, Claessens YE, Doumenc B, et al. Influence of age and renal function on high-sensitivity cardiac troponin T diagnostic accuracy for the diagnosis of acute myocardial infarction. Am J Cardiol. 2013;111(12):1701-7. doi: 10.016/j.amjcard.2013.02.024. Epub Mar 26.

381. Lindahl B, Venge $P$, James $S$. The new high-sensitivity cardiac troponin $T$ assay improves risk assessment in acute coronary syndromes. Am Heart J. 2010;160(2):224-9.

382. Omland T, de Lemos JA, Sabatine MS, Christophi CA, Rice MM, Jablonski KA, et al. A Sensitive Cardiac Troponin T Assay in Stable Coronary Artery Disease. New England Journal of Medicine. 2009;361(26):2538-47. 
383. Latini R, Masson S, Anand IS, Missov E, Carlson M, Vago T, et al. Prognostic value of very low plasma concentrations of troponin $\mathrm{T}$ in patients with stable chronic heart failure. Circulation. 2007;116(11):1242-9.

384. Alam A, Palumbo A, Mucsi I, Barre PE, Sniderman AD. Elevated troponin I levels but not low grade chronic inflammation is associated with cardiac-specific mortality in stable hemodialysis patients. BMC Nephrol. 2013;14:247.(doi):10.1186/471-2369-14-247.

385. de Lemos JA, Drazner MH, Omland T, Ayers CR, Khera A, Rohatgi A, et al. Association of troponin $T$ detected with a highly sensitive assay and cardiac structure and mortality risk in the general population. Jama. 2010;304(22):2503-12. Epub 2010/12/09.

386. Zouridakis E, Avanzas P, Arroyo-Espliguero R, Fredericks S, Kaski JC. Markers of inflammation and rapid coronary artery disease progression in patients with stable angina pectoris. Circulation. 2004;110(13):1747-53.

387. Zebrack JS, Anderson JL, Maycock CA, Horne BD, Bair TL, Muhlestein JB, et al. Usefulness of high-sensitivity $\mathrm{C}$-reactive protein in predicting long-term risk of death or acute myocardial infarction in patients with unstable or stable angina pectoris or acute myocardial infarction. American Journal of Cardiology. 2002;89(2):145-9.

388. Ridker PM. Clinical application of C-reactive protein for cardiovascular disease detection and prevention. Circulation. 2003;107(3):363-9. Epub 2003/01/29.

389. Apple FS, Murakami MM, Pearce LA, Herzog CA. Multi-biomarker risk stratification of $\mathrm{N}$ terminal pro-B-type natriuretic peptide, high-sensitivity $\mathrm{C}$-reactive protein, and cardiac troponin $\mathrm{T}$ and I in end-stage renal disease for all-cause death. Clinical chemistry. 2004;50(12):2279-85.

390. Koenig W. High-sensitivity C-reactive protein and atherosclerotic disease: from improved risk prediction to risk-guided therapy. Int J Cardiol. 2013;168(6):5126-34. Epub 2013/08/28.

391. Mukoyama M, Nakao K, Hosoda K, Suga S, Saito Y, Ogawa Y, et al. Brain natriuretic peptide as a novel cardiac hormone in humans. Evidence for an exquisite dual natriuretic peptide system, atrial natriuretic peptide and brain natriuretic peptide. J Clin Invest. 1991;87(4):1402-12. Epub 1991/04/01.

392. Yeh HM, Lau HP, Lin JM, Sun WZ, Wang MJ, Lai LP. Preoperative plasma N-terminal pro-brain natriuretic peptide as a marker of cardiac risk in patients undergoing elective non-cardiac surgery. $\mathrm{Br} \mathrm{J}$ Surg. 2005;92(8):1041-5. Epub 2005/07/06.

393. Feringa $\mathrm{HH}, \mathrm{Bax} J \mathrm{~J}$, Elhendy A, de Jonge R, Lindemans J, Schouten O, et al. Association of plasma $\mathrm{N}$-terminal pro-B-type natriuretic peptide with postoperative cardiac events in patients undergoing surgery for abdominal aortic aneurysm or leg bypass. Am J Cardiol. 2006;98(1):111-5. Epub 2006/06/21. 394. Feringa HH, Schouten O, Dunkelgrun M, Bax JJ, Boersma E, Elhendy A, et al. Plasma N-terminal pro-B-type natriuretic peptide as long-term prognostic marker after major vascular surgery. Heart. 2007;93(2):226-31. Epub 2006/08/18.

395. Lee TH, Marcantonio ER, Mangione CM, Thomas EJ, Polanczyk CA, Cook EF, et al. Derivation and prospective validation of a simple index for prediction of cardiac risk of major noncardiac surgery. Circulation. 1999;100(10):1043-9.

396. Reeves BC, Ascione R, Chamberlain MH, Angelini GD. Effect of body mass index on early outcomes in patients undergoing coronary artery bypass surgery. J Am Coll Cardiol. 2003;42(4):668-76. 397. Bagnall NM, Faiz O, Darzi A, Athanasiou T. What is the utility of preoperative frailty assessment for risk stratification in cardiac surgery? Interact Cardiovasc Thorac Surg. 2013;17(2):398-402.

398. Prinssen M, Verhoeven ELG, Buth J, Cuypers PWM, van Sambeek MRHM, Balm R, et al. A randomized trial comparing conventional and endovascular repair of abdominal aortic aneurysms. New England Journal of Medicine. 2004;351(16):1607-18.

399. Lederle FA, Freischlag JA, Kyriakides TC, Padberg FT, Matsumura JS, Kohler TR, et al. Outcomes Following Endovascular vs Open Repair of Abdominal Aortic Aneurysm A Randomized Trial. Jama-J Am Med Assoc. 2009;302(14):1535-42.

400. Robless PA, Okonko D, Lintott P, Mansfield AO, Mikhailidis DP, Stansby GP. Increased platelet aggregation and activation in peripheral arterial disease. Eur J Vasc Endovasc Surg. 2003;25(1):16-22. Epub 2003/01/15.

401. Passacquale G, Vamadevan P, Pereira L, Hamid C, Corrigall V, Ferro A. Monocyte-Platelet Interaction Induces a Pro-Inflammatory Phenotype in Circulating Monocytes. PLoS ONE. 2011;6(10):e25595.

402. Tapp LD, Shantsila E, Wrigley BJ, Pamukcu B, Lip GYH. The CD14++CD16+ monocyte subset and monocyte-platelet interactions in patients with ST-elevation myocardial infarction. Journal of Thrombosis and Haemostasis. 2012;10(7):1231-41. 
403. Harding SA, Sarma J, Din JN, Maciocia PM, Newby DE, Fox KA. Clopidogrel reduces plateletleucocyte aggregation, monocyte activation and RANTES secretion in type 2 diabetes mellitus. Heart. 2006;92(9):1335-7. Epub 2006/08/16.

404. Li N. Platelet-lymphocyte cross-talk. J Leukoc Biol. 2008;83(5):1069-78. Epub 2008/01/17.

405. Elzey BD, Tian J, Jensen RJ, Swanson AK, Lees JR, Lentz SR, et al. Platelet-mediated modulation of adaptive immunity. A communication link between innate and adaptive immune compartments. Immunity. 2003;19(1):9-19. Epub 2003/07/23.

406. Elalamy I, Chakroun T, Gerotziafas GT, Petropoulou A, Robert F, Karroum A, et al. Circulating platelet-leukocyte aggregates: a marker of microvascular injury in diabetic patients. Thromb Res. 2008;121(6):843-8. Epub 2007/09/11.

407. Turgut B, Turgut N, Celik Y, Tekgunduz E, Pamuk GE, Demir M. Differences in platelet-leukocyte aggregates among subtypes of acute cerebral ischemia. J Neurol Sci. 2011;305(1-2):126-30.

408. Xiao ZH, Theroux P. Clopidogrel inhibits platelet-leukocyte interactions and thrombin receptor agonist peptide-induced platelet activation in patients with an acute coronary syndrome. J Am Coll Cardiol. 2004;43(11):1982-8.

409. Aukrust $P$, Muller F, Ueland T, Berget $T$, Aaser E, Brunsvig A, et al. Enhanced levels of soluble and membrane-bound CD40 ligand in patients with unstable angina. Possible reflection of T lymphocyte and platelet involvement in the pathogenesis of acute coronary syndromes. Circulation. 1999;100(6):614-20. Epub 1999/08/10.

410. Danese $S$, de la Motte $C$, Reyes BMR, Sans M, Levine AD, Fiocchi C. Cutting edge: T cells trigger CD40-dependent platelet activation and granular RANTES release: A novel pathway for immune response amplification. J Immunol. 2004;172(4):2011-5.

411. Li N, Ji Q, Hjemdahl P. Platelet-lymphocyte conjugation differs between lymphocyte subpopulations. Journal of Thrombosis and Haemostasis. 2006;4(4):874-81.

412. Ridker PM, Rifai N, Pfeffer MA, Sacks F, Braunwald E. Long-term effects of pravastatin on plasma concentration of C-reactive protein. The Cholesterol and Recurrent Events (CARE) Investigators. Circulation. 1999;100(3):230-5. Epub 1999/07/21.

413. Yang DH, Tan N, He PC, Liu Y, Wen JY, Chen JY, et al. [Increased platelet-leukocyte aggregates in patients with acute coronary syndrome]. Zhonghua xin xue guan bing za zhi. 2012;40(6):482-6. Epub 2012/09/05.

414. Rinder HM, Bonan JL, Rinder CS, Ault KA, Smith BR. Dynamics of leukocyte-platelet adhesion in whole blood. Blood. 1991;78(7):1730-7. Epub 1991/10/01.

415. Harding SA, Din JN, Sarma J, Jessop A, Weatherall M, Fox KA, et al. Flow cytometric analysis of circulating platelet-monocyte aggregates in whole blood: methodological considerations. Thromb Haemost. 2007;98(2):451-6. Epub 2007/08/28.

416. Zhou X, Nicoletti A, Elhage R, Hansson GK. Transfer of CD4(+) T cells aggravates atherosclerosis in immunodeficient apolipoprotein E knockout mice. Circulation. 2000;102(24):2919-22. Epub 2000/01/11.

417. Zhou XH, Robertson AKL, Rudling M, Parini P, Hansson GK. Lesion development and response to immunization reveal a complex role for CD4 in atherosclerosis. Circulation Research. 2005;96(4):42734.

418. Ammirati E, Cianflone D, Vecchio V, Banfi M, Vermi AC, De Metrio M, et al. Effector Memory T cells Are Associated With Atherosclerosis in Humans and Animal Models. Journal of the American Heart Association. 2012;1(1):27-41. Epub 2012/11/07.

419. Bacon KB, Premack BA, Gardner P, Schall TJ. Activation of Dual T-Cell Signaling Pathways by the Chemokine Rantes. Science. 1995;269(5231):1727-30.

420. Acres RB, Lamb JR, Feldman M. Effects of Platelet-Derived Growth-Factor and Epidermal Growth-Factor on Antigen-Induced Proliferation of Human T-Cell Lines. Immunology. 1985;54(1):9-16. 421. $\mathrm{Li} \mathrm{N}$. CD4+ $\mathrm{T}$ cells in atherosclerosis: regulation by platelets. Thromb Haemost. 2013;109(6):980-90. Epub 2013/03/01.

422. Hebel K, Rudolph M, Kosak B, Chang HD, Butzmann J, Brunner-Weinzierl MC. IL-1beta and TGFbeta act antagonistically in induction and differentially in propagation of human proinflammatory precursor CD4+ T cells. J Immunol. 2011;187(11):5627-35. Epub 2011/11/04.

423. Gerdes N, Zhu L, Ersoy M, Hermansson A, Hjemdahl P, Hu H, et al. Platelets regulate CD4(+) Tcell differentiation via multiple chemokines in humans. Thromb Haemost. 2011;106(2):353-62. Epub 2011/06/10. 
424. Taub DD, Turcovski-Corrales SM, Key ML, Longo DL, Murphy WJ. Chemokines and T lymphocyte activation: I. Beta chemokines costimulate human T lymphocyte activation in vitro. J Immunol. 1996;156(6):2095-103. Epub 1996/03/15.

425. Zhou L, Lopes JE, Chong MM, Ivanov, II, Min R, Victora GD, et al. TGF-beta-induced Foxp3 inhibits $T(H) 17$ cell differentiation by antagonizing RORgammat function. Nature. 2008;453(7192):23640. Epub 2008/03/28.

426. Liu CY, Battaglia M, Lee SH, Sun QH, Aster RH, Visentin GP. Platelet factor 4 differentially modulates CD4+CD25+ (regulatory) versus CD4+CD25- (nonregulatory) T cells. J Immunol. 2005;174(5):2680-6. Epub 2005/02/25.

427. Kyaw T, Winship A, Tay C, Kanellakis P, Hosseini H, Cao A, et al. Cytotoxic and proinflammatory CD8+ T lymphocytes promote development of vulnerable atherosclerotic plaques in apoE-deficient mice. Circulation. 2013;127(9):1028-39. Epub 2013/02/12.

428. Kronenberg M, Gapin L. The unconventional lifestyle of NKT cells. Nat Rev Immunol. 2002;2(8):557-68. Epub 2002/08/03.

429. Braun NA, Covarrubias R, Major AS. Natural killer T cells and atherosclerosis: form and function meet pathogenesis. Journal of innate immunity. 2010;2(4):316-24. Epub 2010/04/09.

430. Nieswandt B, Hafner M, Echtenacher B, Mannel DN. Lysis of tumor cells by natural killer cells in mice is impeded by platelets. Cancer Res. 1999;59(6):1295-300.

431. Steinhubl SR, Badimon JJ, Bhatt DL, Herbert JM, Luscher TF. Clinical evidence for antiinflammatory effects of antiplatelet therapy in patients with atherothrombotic disease. Vasc Med. 2007;12(2):113-22. Epub 2007/07/10.

432. Altman DG. Practical Statistics For Medical Research1991.

433. Tellides G, Tereb DA, Kirkiles-Smith NC, Kim RW, Wilson JH, Schechner JS, et al. Interferongamma elicits arteriosclerosis in the absence of leukocytes. Nature. 2000;403(6766):207-11. Epub 2000/01/26.

434. Gerdes N, Zhu L, Ersoy M, Hermansson A, Hjemdahl P, Hu H, et al. Platelets regulate CD4 T-cell differentiation via multiple chemokines in humans. Thromb Haemost. 2011;106(2):353-62. Epub 2011/06/10.

435. Fleischer J, Grage-Griebenow E, Kasper B, Heine H, Ernst M, Brandt E, et al. Platelet factor 4 inhibits proliferation and cytokine release of activated human T cells. J Immunol. 2002;169(2):770-7. Epub 2002/07/05.

436. Kabashima K, Murata T, Tanaka H, Matsuoka T, Sakata D, Yoshida N, et al. Thromboxane A2 modulates interaction of dendritic cells and $\mathrm{T}$ cells and regulates acquired immunity. Nat Immunol. 2003;4(7):694-701. Epub 2003/06/05.

437. Calabresse C, Nguer MC, Pellegrini O, Benveniste J, Richard Y, Thomas Y. Induction of highaffinity paf receptor expression during T cell activation. Eur J Immunol. 1992;22(6):1349-55. Epub 1992/06/01.

438. Frostegard J, Huang YH, Ronnelid J, Schafer-Elinder L. Platelet-activating factor and oxidized LDL induce immune activation by a common mechanism. Arterioscler Thromb Vasc Biol. 1997;17(5):963-8. Epub 1997/05/01.

439. Lindemann S, Tolley ND, Dixon DA, Mclntyre TM, Prescott SM, Zimmerman GA, et al. Activated platelets mediate inflammatory signaling by regulated interleukin 1 beta synthesis. J Cell Biol. 2001;154(3):485-90.

440. Laurat E, Poirier B, Tupin E, Caligiuri G, Hansson GK, Bariety J, et al. In vivo downregulation of $\mathrm{T}$ helper cell 1 immune responses reduces atherogenesis in apolipoprotein E-knockout mice. Circulation. 2001;104(2):197-202.

441. Buono C, Binder CJ, Stavrakis G, Witztum JL, Glimcher LH, Lichtman AH. T-bet deficiency reduces atherosclerosis and alters plaque antigen-specific immune responses. Proc Natl Acad Sci U S A. 2005;102(5):1596-601.

442. Bettelli E, Korn T, Oukka M, Kuchroo VK. Induction and effector functions of $\mathrm{T}(\mathrm{H}) 17$ cells. Nature. 2008;453(7198):1051-7.

443. van Es T, van Puijvelde GHM, Ramos OH, Segers FME, Joosten LA, van den Berg WB, et al. Attenuated atherosclerosis upon IL-17R signaling disruption in LDLr deficient mice. Biochem Biophys Res Commun. 2009;388(2):261-5.

444. Acosta-Rodriguez EV, Napolitani G, Lanzavecchia A, Sallusto F. Interleukins 1beta and 6 but not transforming growth factor-beta are essential for the differentiation of interleukin 17-producing human T helper cells. Nat Immunol. 2007;8(9):942-9. Epub 2007/08/07. 
445. Drolet AM, Thivierge M, Turcotte S, Hanna D, Maynard B, Stankova J, et al. Platelet-activating factor induces Th17 cell differentiation. Mediators of inflammation. 2011;2011:913802. Epub 2011/10/21.

446. Ganesh BB, Bhattacharya P, Gopisetty A, Sheng J, Vasu C, Prabhakar BS. IL-1beta promotes TGF-beta1 and IL-2 dependent Foxp3 expression in regulatory T cells. PLoS ONE. 2011;6(7):e21949. Epub 2011/07/23.

447. Bacon KB, Premack BA, Gardner P, Schall TJ. Activation of dual T cell signaling pathways by the chemokine RANTES. Science. 1995;269(5231):1727-30. Epub 1995/09/22.

448. Thomas DW, Rocha PN, Nataraj C, Robinson LA, Spurney RF, Koller BH, et al. Proinflammatory actions of thromboxane receptors to enhance cellular immune responses. J Immunol. 2003;171(12):6389-95. Epub 2003/12/10.

449. Vivier E, Deryckx S, Wang JL, Valentin H, Peronne C, de Vries JE, et al. Immunoregulatory functions of paf-acether. VI. Inhibition of T cell activation via CD3 and potentiation of T cell activation via CD2. Int Immunol. 1990;2(6):545-53.

450. Gajewski TF, Fitch FW. Anti-proliferative effect of IFN-gamma in immune regulation. . The Journal of Immunology. 1988;140(12):4245-52.

451. Grakoui A, Bromley SK, Sumen C, Davis MM, Shaw AS, Allen PM, et al. The immunological synapse: a molecular machine controlling T cell activation. Science. 1999;285(5425):221-7. Epub 1999/07/10.

452. Benson RA, Adamson K, Corsin-Jimenez M, Marley JV, Wahl KA, Lambs JR, et al. Notch1 colocalizes with CD4 on activated T cells and Notch signaling is required for IL-10 production. Eur J Immunol. 2005;35(3):859-69.

453. Evangelista V, Smyth SS. Chapter 16 - Interactions Between Platelets, Leukocytes and the Endothelium. In: Michelson AD, editor. Platelets (Third Edition): Academic Press; 2013. p. 295-312.

454. Price MJ. Bedside evaluation of thienopyridine antiplatelet therapy. Circulation. 2009;119(19):2625-32. Epub 2009/05/20.

455. Collet JP, Hulot JS, Pena A, Villard E, Esteve JB, Silvain J, et al. Cytochrome P450 2 C19 polymorphism in young patients treated with clopidogrel after myocardial infarction: a cohort study. Lancet. 2009;373(9660):309-17. Epub 2008/12/26.

456. Mega JL, Close SL, Wiviott SD, Shen L, Hockett RD, Brandt JT, et al. Cytochrome p-450 polymorphisms and response to clopidogrel. N Engl J Med. 2009;360(4):354-62. Epub 2008/12/25.

457. Bhatt DL, Pare G, Eikelboom JW, Simonsen KL, Emison ES, Fox KAA, et al. The relationship between CYP2C19 polymorphisms and ischaemic and bleeding outcomes in stable outpatients: the CHARISMA genetics study. European Heart Journal. 2012;33(17):2143-50.

458. Schror K, Siller-Matula JM, Huber K. Pharmacokinetic basis of the antiplatelet action of prasugrel. Fundam Clin Pharmacol. 2012;26(1):39-46. Epub 2011/09/08.

459. Aradi D, Komocsi A, Price MJ, Cuisset T, Ari H, Hazarbasanov D, et al. Efficacy and safety of intensified antiplatelet therapy on the basis of platelet reactivity testing in patients after percutaneous coronary intervention: systematic review and meta-analysis. Int J Cardiol. 2013;167(5):2140-8. Epub 2012/06/19.

460. Burdess A, Nimmo AF, Campbell N, Harding SA, Garden OJ, Dawson AR, et al. Perioperative platelet and monocyte activation in patients with critical limb ischemia. J Vasc Surg. 2010;52(3):697703. Epub 2010/09/08.

461. Kertai MD, Boersma E, Klein J, Van Urk H, Bax JJ, Poldermans D. Long-term prognostic value of asymptomatic cardiac troponin $\mathrm{T}$ elevations in patients after major vascular surgery. Eur J Vasc Endovasc Surg. 2004;28(1):59-66. Epub 2004/06/05.

462. Levy M, Heels-Ansdell D, Hiralal R, Bhandari M, Guyatt G, Yusuf S, et al. Prognostic value of troponin and creatine kinase muscle and brain isoenzyme measurement after noncardiac surgery: a systematic review and meta-analysis. Anesthesiology. 2011;114(4):796-806. Epub 2011/02/22.

463. Brenchley JM, Douek DC, Ambrozak DR, Chatterji M, Betts MR, Davis LS, et al. Expansion of activated human naive T-cells precedes effector function. Clinical and experimental immunology. 2002;130(3):432-40. Epub 2002/11/28.

464. Goubran HA, Burnouf T, Radosevic M, El-Ekiaby M. The platelet-cancer loop. Eur J Intern Med. 2013;24(5):393-400.

465. Vincent JL, Yagushi A, Pradier O. Platelet function in sepsis. Critical care medicine. 2002;30(5 Suppl):S313-7. Epub 2002/05/11. 


\section{REFERENCES}

466. Akinosoglou K, Alexopoulos D. Use of antiplatelet agents in sepsis: A glimpse into the future. Thrombosis Research. 2014;133(2):131-8. 
${ }_{11}$ Appendices 


\subsection{Appendix 1 - Buffers and Solutions}

PBS

$\mathrm{NaCl}$ $145 \mathrm{mM}$

$\mathrm{Na}_{2} \mathrm{HPO}_{4}$

$8.7 \mathrm{mM}$

$\mathrm{NaH}_{2} \mathrm{PO}_{4}$

$1.3 \mathrm{mM}$

In $\mathrm{dd}_{2} \mathrm{O}$, autoclaved strilised

FACS buffer (v/v)

Fetal Calf Serum (FCS)

$2 \%$

Sodium azide

$0.1 \%$

PBS

$97.9 \%$

Complete T cell media (CTCM) (v/v)

Dulbecco's Modified Eagle Medium

$85.9 \%$

FCS

$10 \%$

L-glutamate (200 mM)

$1 \%$

Penicillin/Streptomycin

$1 \%(100 \mathrm{U} / \mathrm{ml} / 10 \mathrm{mg} / \mathrm{ml})$

HEPES buffer (1 M)

$1 \%$

B-Mecaptoethanol

$0.1 \%$

Non-essential amino acids (10nM)

$0.1 \%$

Filter sterilized using $0.22 \mu \mathrm{m}$ syringe filter.

Isolation Buffer (IB) (v/v)

PBS

$97.8 \%$

FCS

$2 \%$

$\operatorname{EDTA}(0.5 \mathrm{M})$

$0.2 \%$ 
11.2 Appendix 2 - Antibody information

\begin{tabular}{|c|c|c|c|c|}
\hline $\begin{array}{l}\text { Cell Surface } \\
\text { Markers }\end{array}$ & Flurophore & Clone & Isotype & Manufacturer \\
\hline CD3 & FITC & HIT3a & Mouse IgG2a & Biolegend \\
\hline CD8 & $\begin{array}{l}\text { PE } \\
\text { PECy7 }\end{array}$ & $\begin{array}{l}\text { HIT8a } \\
\text { RPA-T8 }\end{array}$ & $\begin{array}{l}\text { Mouse IgG1 } \\
\text { Mouse IgG1 }\end{array}$ & $\begin{array}{l}\text { Biolegend } \\
\text { BD Bioscience }\end{array}$ \\
\hline CD42a & PerCP & Beb-1 & Mouse lgG1 & BD Bioscience \\
\hline CD4 & PECy7 & OKT4 & Mouse IgG2a & Biolegend \\
\hline CD25 & APC & BC96 & Mouse IgG1 & Biolegend \\
\hline CD44 & PE & BJ18 & Mouse IgG1 & Biolegend \\
\hline CD62L & APC & DREG-56 & Mouse IgG1 & Biolegend \\
\hline CD56 & $\mathrm{PE}$ & HCD56 & Mouse IgG1 & Biolegend \\
\hline \multirow[t]{5}{*}{ Isotype control } & FITC & MOPC-173 & Mouse IgG2a & Biolegend \\
\hline & $\mathrm{PE}$ & MOPC-21 & Mouse IgG1 & Biolegend \\
\hline & PerCP & MOPC-21 & Mouse IgG1 & BD Bioscience \\
\hline & PECy7 & MPC-11 & Mouse IgG2b & Biolegend \\
\hline & APC & MOPC-21 & Mouse IgG1 & Biolegend \\
\hline $\begin{array}{l}\text { Intracellular } \\
\text { marker }\end{array}$ & Flurophore & Clone & Isotype & Manufacturer \\
\hline IFNy & FITC & 25723.11 & Mouse IgG2a & BD Bioscience \\
\hline IL-17A & PE & N49-653 & Mouse IgG1 & BD Bioscience \\
\hline FoxP3 & Alexa Fluor 647 & 259D/C7 & Mouse IgG1 & BD Bioscience \\
\hline \multirow[t]{3}{*}{ Isotype control } & FITC & MOPC-173 & Mouse IgG2a & Biolegend \\
\hline & $\mathrm{PE}$ & MOPC-21 & Mouse IgG1 & BD Bioscience \\
\hline & Alexa Fluor 647 & MOPC-21 & Mouse IgG1 & BD Bioscience \\
\hline
\end{tabular}




\subsection{Appendix 3 - Supplementary graphs for chapter 8}

A

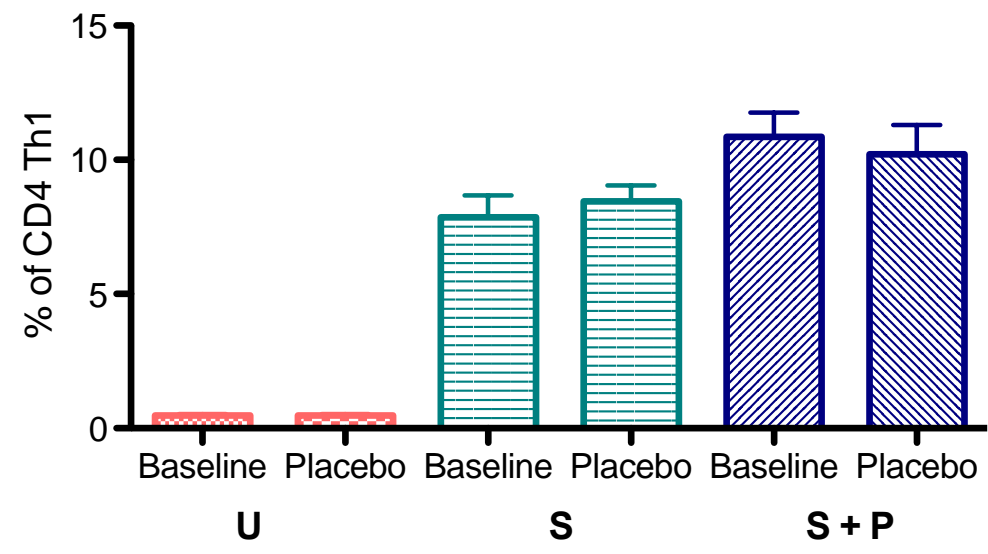

B

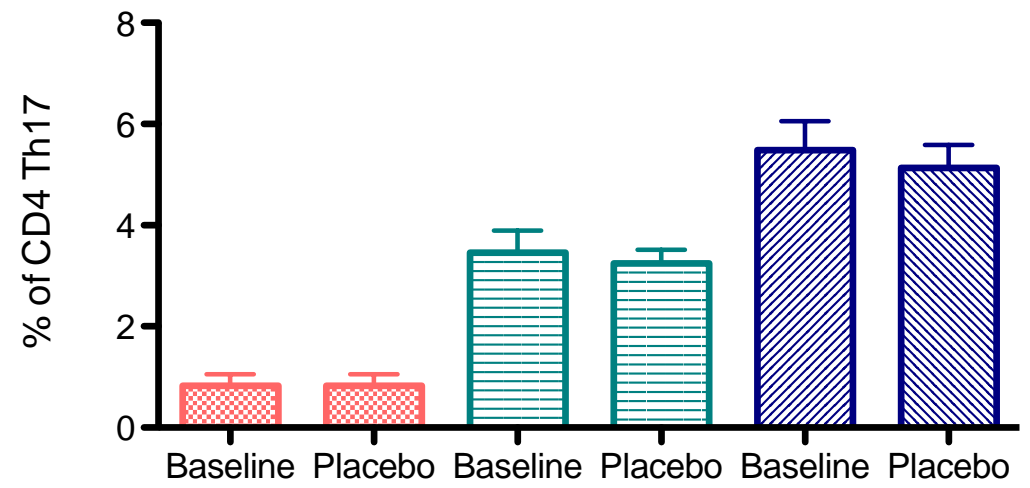

$\mathbf{U}$

S

S + P

C

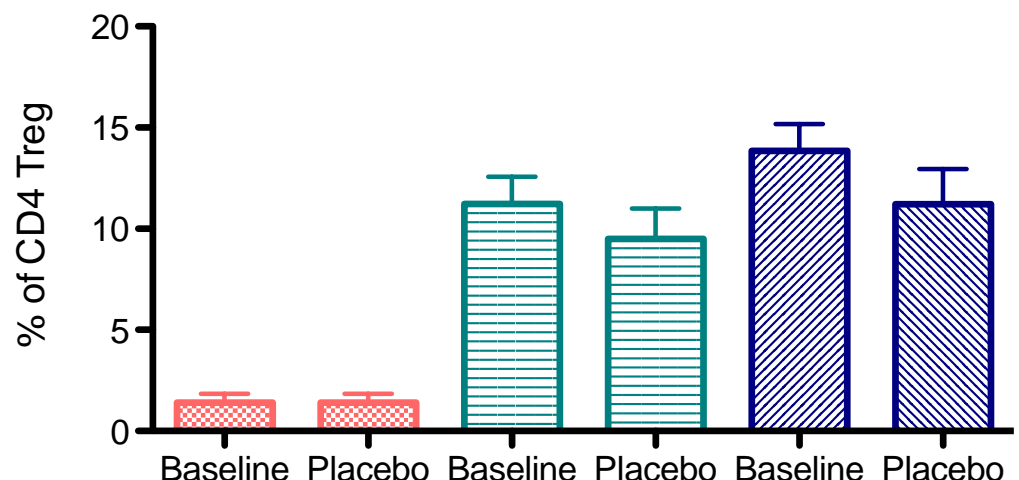

$\mathrm{U}$

$S$

$\mathbf{S}+\mathbf{P}$

Figure 11-1 No difference in isolated CD4 T cell differentiation following placebo therapy compared to baseline in all culture conditions

Isolated CD4 T cells at baseline and after placebo therapy were cultured without stimulation $(\mathrm{U})$, with anti-CD3/CD28 stimulation (S) and stimulation plus autologous platelets (S + P). CD4 T cell subsets were measured by intracellular cytokine staining for IFN-Y (Th1), IL-17A (Th17) and FoxP3 (Treg) using flow cytometry. Data is displayed as mean \pm SEM for the proportion of Th1 (A), Th17 (B) and Treg subsets (C). The data represents all 12 patients. Paired T tests of culture conditions were found to be not significant. 